MILTON SAULO RAIMUNDO

DESENVOLVIMENTO DE UM MODELO ADAPTATIVO BASEADO EM UM SISTEMA SVR-WAVELET HIIBRIDO PARA PREVISÃO DE SÉRIES TEMPORAIS FINANCEIRAS 
MILTON SAULO RAIMUNDO

DESENVOLVIMENTO DE UM MODELO ADAPTATIVO BASEADO EM UM SISTEMA SVR-WAVELET HIIBRIDO PARA PREVISÃO DE SÉRIES TEMPORAIS FINANCEIRAS

Tese apresentada à Escola Politécnica da Universidade de São Paulo para obtenção do título de doutor em Ciências

São Paulo 
MILTON SAULO RAIMUNDO

\section{DESENVOLVIMENTO DE UM MODELO ADAPTATIVO BASEADO EM UM SISTEMA SVR-WAVELET HIIBRIDO PARA PREVISÃO DE SÉRIES TEMPORAIS FINANCEIRAS}

Tese apresentada à Escola Politécnica da Universidade de São Paulo para obtenção do título de doutor em Ciências

Área de concentração:

Engenharia de Controle e Automação Mecânica

Orientador:

Jun Okamoto Júnior

São Paulo 


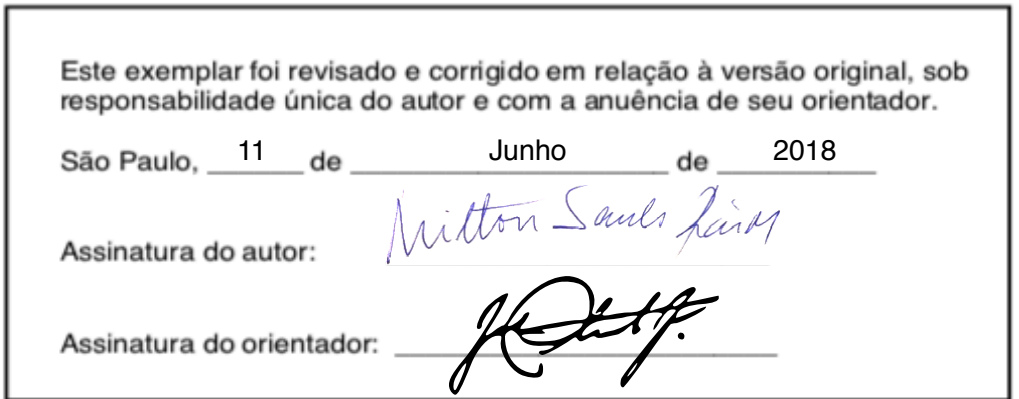

\section{Catalogação-na-publicação}

\section{Raimundo, Milton Saulo}

Desenvolvimento de um modelo adaptativo baseado em um sistema SVR-wavelet híbrido para previsão de séries temporais financeiras / M. S. Raimundo -- versão corr. -- São Paulo, 2018. $144 \mathrm{p}$.

Tese (Doutorado) - Escola Politécnica da Universidade de São Paulo. Departamento de Engenharia Mecatrônica e de Sistemas Mecânicos.

1.SVR 2.wavelets 3.Séries Temporais Financeiras 4.Expoente de Hurst 5.Estatística R/S I.Universidade de São Paulo. Escola Politécnica. Departamento de Engenharia Mecatrônica e de Sistemas Mecânicos II.t. 


\section{DEDICATÓRIA}

À Maria Aparecida Raimundo (in memorian) e à Irene Micucci Raimundo, que dignamente me apresentaram à importância da família e o caminho da honestidade e persistência. À minha esposa Isabel e filhos, Gabriela e Gustavo, pelo apoio incondicional em todos os momentos. Sem vocês nenhuma conquista valeria a pena. Ao meu irmão Nilton Paulo, pela amizade e companheirismo. 


\section{AGRADECIMENTOS}

Primeiramente agradeço a Deus, por me abençoar todos os dias e iluminar meu caminho, fortalecendo-me para seguir em frente.

Ao Prof. Jun Okamoto Jr., o meu reconhecimento pela oportunidade de realizar este trabalho ao lado de alguém que emana sabedoria. Meu respeito e admiração pela sua serenidade e pelo seu Dom no ensino da Ciência.

Aos membros da Secretaria do PPGEM - USP, que direta ou indiretamente contribuíram de alguma forma para a realização deste trabalho, o meu reconhecimento e gratidão. 
Porque eu sou do tamanho do que vejo e não do tamanho da minha altura.

$E$ o que vejo são os meus sonhos

(Alberto Caeiro - heterônimo de Fernando Pessoa) 


\section{RESUMO}

A necessidade de antecipar e identificar variações de acontecimentos apontam para uma nova direção nos mercados de bolsa de valores e vem de encontro às análises das oscilações de preços de ativos financeiros. Esta necessidade leva a argumentar sobre novas alternativas na predição de séries temporais financeiras utilizando métodos de aprendizado de máquinas e vários modelos têm sido desenvolvidos para efetuar a análise e a previsão de dados de ativos financeiros. Este trabalho tem por objetivo propor o desenvolvimento de um modelo de previsão adaptativo baseado em um sistema SVR-wavelet híbrido, que integra modelos de wavelets e Support Vector Regression (SVR) na previsão de séries financeiras. O método consiste na utilização da Transformada de Wavelet Discreta (DWT) a fim de decompor dados de séries de ativos financeiros que são utilizados como variáveis de entrada do SVR com o objetivo de prever dados futuros de ativos financeiros. O modelo proposto é aplicado a um conjunto de ativos financeiros do tipo Foreign Exchange Market (FOREX), Mercado Global de Câmbio, obtidos a partir de uma base de conhecimento público. As séries são ajustadas gerando-se novas predições das séries originais, que são comparadas com outros modelos tradicionais tais como o modelo Autorregressivo Integrado de Médias Móveis (ARIMA), o modelo Autorregressivo Fracionário Integrado de Médias Móveis (ARFIMA), o modelo Autorregressivo Condicional com Heterocedasticidade Generalizado $(\mathrm{GARCH})$ e o modelo SVR tradicional com Kernel. Além disso, realizam-se testes de normalidade e de raiz unitária para distribuição não linear, tal como testes de correlação, para constatar que as séries temporais FOREX são adequadas para a comprovação do modelo híbrido SVR-wavelet e posterior comparação com modelos tradicionais. Verifica-se também a aderência ao Expoente de Hurst por meio da estatística de Reescalonamento (R/S).

Palavras-chave: SVR, wavelets, Séries Temporais Financeiras, Expoente de Hurst, Estatística $\mathrm{R} / \mathrm{S}$. 


\section{ABSTRACT}

The necessity to anticipate and identify changes in events points to a new direction in the stock exchange market and reaches the analysis of the oscillations of prices of financial assets. This necessity leads to an argument about new alternatives in the prediction of financial time series using machine learning methods. Several models have been developed to perform the analysis and prediction of financial asset data. This thesis aims to propose the development of SVR-wavelet model, an adaptive and hybrid prediction model, which integrates wavelet models and Support Vector Regression (SVR), for prediction of Financial Time Series, particularly Foreign Exchange Market (FOREX), obtained from a public knowledge base. The method consists of using the Discrete Wavelets Transform (DWT) to decompose data from FOREX time series, that are used as SVR input variables to predict new data. The series are adjusted by generating new predictions of the original series, which are compared with other traditional models such as the Autoregressive Integrated Moving Average model (ARIMA), the Autoregressive Fractionally Integrated Moving Average model (ARFIMA), the Generalized Autoregressive Conditional Heteroskedasticity model (GARCH) and the traditional SVR model with Kernel. In addition, normality and unit root tests for non-linear distribution, and correlation tests, are performed to verify that the FOREX time series are adequate for the verification of SVR-wavelet hybrid model and comparison with traditional models. There is also the adherence to the Hurst Exponent through the statistical Rescaled Range (R/S).

Keywords: SVR, wavelets, Financial Time Series, Hurst Exponent, Statistical R/S 


\section{LISTA DE ILUSTRAÇÕES}

Figura 1 Função wavelet $\operatorname{Haar} \Psi^{H}(t) . \quad \ldots \ldots \ldots$. . . . . . . . . . . . . . . . . . 37

Figura 2 Representação de um hiperplano de separação onde se destacam os

\begin{tabular}{|c|}
\hline vetores de suporte, as distâncias $\frac{b}{\|w\|}$ e $\frac{2}{\|w\|}$ e as regiões $w \cdot x+b>0$ \\
\hline \hline e $w \cdot x+b<0$
\end{tabular}

Figura 3 Representação de um hiperplano de separação onde se evidenciam os vetores de suporte e a variável de folga $\xi$, conforme a equação 47. . 42

Figura 4 Ilustração do conjunto de dados não lineares (a), da fronteira não linear no espaço de entradas (b) e da fronteira linear no espaço de características (c) (96). . . . . . . . . . . . . . . . . . . . . . . . . 43

Figura 5 Técnicas de denoising ou wavelet shrinkage (129). . . . . . . . . . . . . 52

Figura 6 Efeito das modificações proporcionadas pelos parâmetros (a) e (b) sob a função wavelet mãe (129). . . . . . . . . . . . . . . . . . . . . 52

Figura 7 Famílias de wavelet Haar, Daubechie de ordem 4 (db4), Coiflet de ordem 4 (coif4) e Gaussian de ordem 4 (gaus4) obtidas por meio do software MATLAB. . . . . . . . . . . . . . . . . . . . . . . 54

Figura $8 \quad$ Modelo proposto para predição de séries temporais por meio do modelo SVR-wavelet híbrido. . . . . . . . . . . . . . . . . . . . . 55

Figura 9 Representação da metodologia adotada na predição de séries temporais por meio do modelo SVR-wavelet híbrido.

Figura 10 Gráficos contendo as séries históricas dos preços do ativo FOREX AUD-

\begin{tabular}{|l|}
\hline JPY com periodicidades de 15 minutos, 1 hora e 1 dia, com a \\
\hline aplicação de técnicas de denoising ou wavelet shrinkage para elimi- \\
\hline nação de ruídos contidos na série. Somente os últimos 2048 pontos \\
\hline são amostrados.
\end{tabular}

Figura 11 Macro fluxograma das principais tarefas no processo de montagem e execução dos testes.

Figura 12 Gráfico de dispersão para a relação entre as moedas AUD e JPY com periodicidade de 15 minutos. . . . . . . . . . . . . . . . 87

Figura $13 \quad$ Gráficos sobrepostos da série prevista $(\times)$, obtido por meio dos méto-

dos svm e predict, e da série original $(\bullet)$ para a relação entre as
moedas AUD e JPY com periodicidade de 15 minutos. 
Figura $14 \quad$ Gráficos sobrepostos da série original $(\bigcirc)$ e sua Transformada wave-

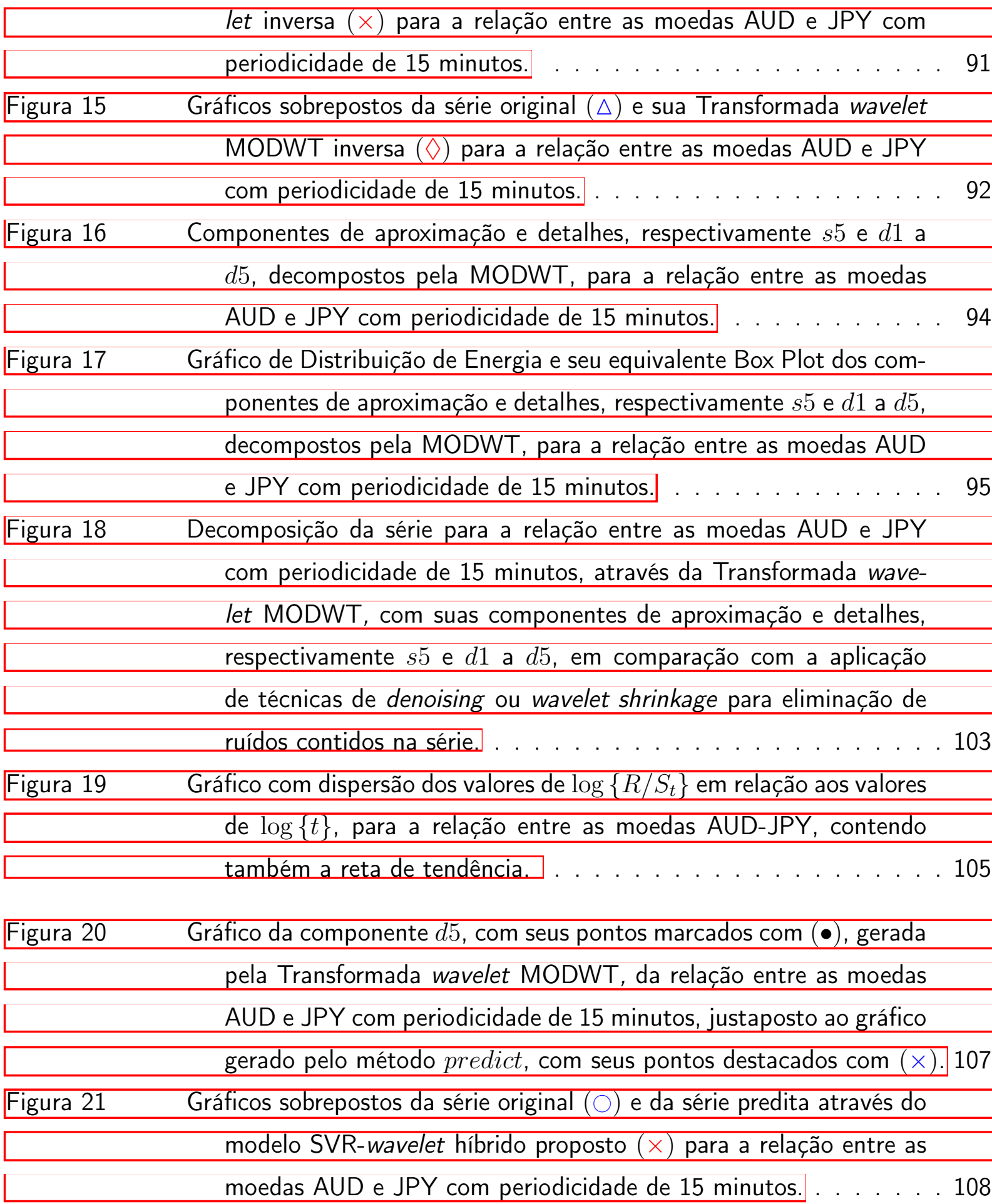




\section{LISTA DE TABELAS}

Tabela 1 Interpretação admissível dos p-values (3) . . . . . . . . . . . . . . . 66

Tabela 2 Resultados dos p-values dos métodos para testes de Normalidade. . . 84

Tabela $3 \quad$ Resultados dos p-values dos métodos para testes de Raiz Unitária. . . 85

Tabela $4 \quad$ Resultados dos p-values dos métodos para testes de Não-Linearidade. 86

Tabela $5 \quad$ Resultados das métricas MSE, RMSE, MAE, MPE e MAPE, calculados

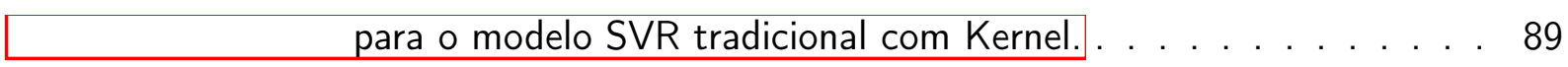

Tabela $6 \quad$ Resultados das métricas MASE, ACF1, AIC, AICc e BIC, calculados para o modelo SVR tradicional com Kernel. . . . . . . . . . . . . . 90

Tabela $7 \quad$ Resultados das métricas MSE, RMSE, MAE, MPE e MAPE, calculados para o modelo híbrido SVR-wavelet. . . . . . . . . . . . . . . 97

Tabela $8 \quad$ Resultados das métricas MASE, ACF1, AIC, AICc e BIC, calculados para o modelo híbrido SVR-wavelet. . . . . . . . . . . . . . . 98

Tabela 9 Resultados das métricas MSE, RMSE, MAE, MPE e MAPE, calculados

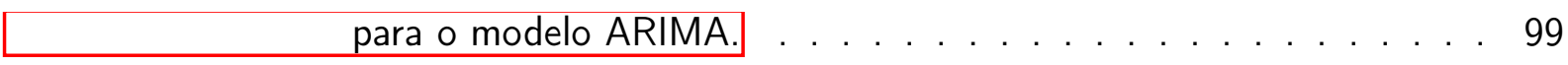

Tabela $10 \quad$ Resultados das métricas MASE, ACF1, AIC, AICc e BIC, calculados para o modelo ARIMA. . . . . . . . . . . . . . . . . . 100

Tabela $11 \quad$ Resultados das métricas MSE, RMSE, MAE, MPE e MAPE, calculados para o modelo ARFIMA. . . . . . . . . . . . . . . . . . . . . . 101

Tabela $12 \quad$ Resultados das métricas MASE, ACF1, AIC, AICc e BIC, calculados

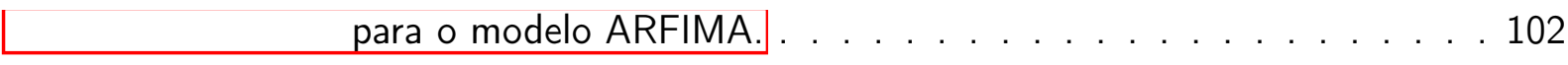

Tabela $13 \quad$ Resultados das métricas AIC e BIC, calculados pelo modelo GARCH. . 104

Tabela $14 \quad$ Valores parciais para a estimativa do expoente de Hurst $(H)$ obtidos

\begin{tabular}{|c|}
\hline por meio das tarefas executadas, conforme descrição no método \\
\hline utilizado, para a relação entre as moedas AUD-JPY com periodi- \\
\hline
\end{tabular}

cidade de 1 em 1 dia . . . . . . . . . . . . . . . . . . . 104

Tabela $15 \quad$ Valores estimados e estatísticas da regressão obtidos por meio das funções $l m$, summary e coef disponíveis no ambiente para análise de dados estatísticos $R$ (47). . . . . . . . . . . . . . . . . . . . . . . 105

Tabela 16 Comparação dos valores dos métodos AIC, AICc e BIC entre o modelo \begin{tabular}{|c|}
\hline ARIMA e o modelo híbrido SVR-wavelet, destacando-se em verde, \\
\hline os menores valores de AIC, AICc e BIC para o modelo híbrido SVR- \\
\hline wavelet.
\end{tabular} 
Tabela 17 Comparação dos valores dos métodos AIC, AICc e BIC entre o modelo ARFIMA e o modelo híbrido SVR-wavelet, destacando-se em verde, os menores valores de AIC, AICc e BIC para o modelo híbrido SVRwavelet. . . . . . . . . . . . . . . . . 110

Tabela 18 Comparação dos valores dos métodos AIC e BIC entre o modelo GARH e o modelo híbrido SVR-wavelet, destacando-se em verde, os menores valores de AIC e BIC para o modelo híbrido SVR-wavelet. . . 111

Tabela 19 Comparação dos valores dos métodos AIC, AICc e BIC entre o modelo SVR tradicional com Kernel e o modelo híbrido SVR-wavelet, destacando-se em verde, os menores valores de AIC, AICc e BIC para o modelo híbrido SVR-wavelet.

Tabela 20 Teste de significância, conforme sugerido por Couillard e Davison, utilizando-se da estatística $p-$ value $<0,001$, obtidos por meio da função t.test disponível no ambiente para análise de dados estatísticos $R(47)$. . . . . . . . . . . . . . . . . . . . . . . . . . 113

Tabela $21 \quad$ Resultados obtidos por meio das funções desenvolvidas no ambiente para análise de dados estatísticos $R(47)$, conforme metodologia desenvolvida por Mandelbrot e Wallis (85), baseada nos trabalhos 
SUMÁRIO

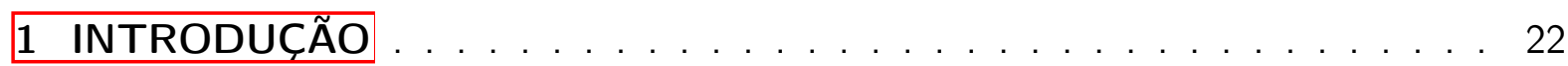

2 REGRESSÃO E MÉTODOS DE APRENDIZADO DE MÁQUINAS . . . . 25

2.1 ALGORITHMIC TRADING . . . . . . . . . . . . . . . . . . 25

2.2 ANÁLISE TÉCNICA E ANÁLISE FUNDAMENTALISTA . . . . . . . . . . . 25

2.2 .1 Análise Técnica . . . . . . . . . . . . . . . . . . . 25

2.2 .2 Análise Fundamentalista . . . . . . . . . . . . . . . . . . 26

2.3 MODELOS MATEMÁTICOS . . . . . . . . . . . . . . . . . . 26

2.3 .1 Modelos Autorregressivos . . . . . . . . . . . . . . . . . . 27

2.3 .2 Modelos de Médias Móveis . . . . . . . . . . . . . . . . 28

2.3 .3 Modelos Autorregressivos e de Médias Móveis . . . . . . . . . . 28

2.3 .4 Modelos Autorregressivos Integrados de Médias Móveis . . . . . . . . 28

2.3.5 Modelos Autorregressivos Fracionários Integrados de Médias Móveis . 29

2.3.6 Modelos Autorregressivos Condicionais com Heterocedasticidade . . . 30

2.3 .7 Modelos ARCH Generalizados . . . . . . . . . . . . . . . . 30

2.4 MODELOS FRACTAIS . . . . . . . . . . . . . . . . . 31

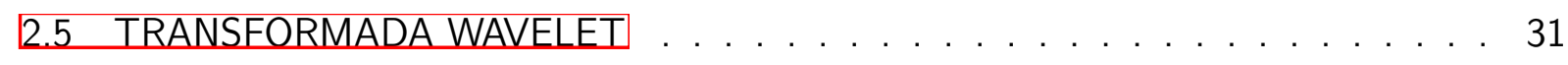

2.5 .1 Análise Multirresolução (MRA) $\ldots \ldots \ldots \ldots$

2.5 .2 Transformada de Wavelet Discreta . . . . . . . . . . . . . 35

2.5 .3 Transformada Wavelet Discreta de Máxima Sobreposição. . . . . . . 35

2.5 .4 Wavelet Haar . . . . . . . . . . . . . . . . . . . . . . . . . 37

2.6 MÉTODOS DE APRENDIZADO DE MÁQUINAS . . . . . . . . . . . 38

2.6 .1 Árvores de Regressão . . . . . . . . . . . . . . . . . . . 38

2.6 .2 Regressão por Redes Neurais $\ldots \ldots . \ldots 38$

2.6 .3 Regressão por Aprendizagem Bayesiana . . . . . . . . . . . . 39

2.6 .4 Regressão por k-Vizinhos mais Próximos . . . . . . . . . . . . . . . . . 39

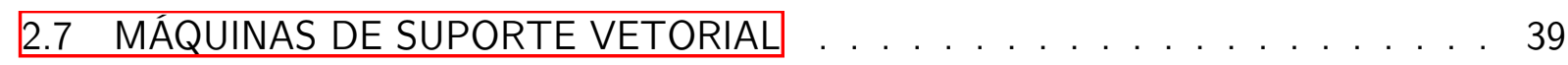

2.7 .1 SVM e as Margens Rígidas . . . . . . . . . . . . . . . . 40

2.7 .2 SVM e as Margens Suaves . . . . . . . . . . . . . . . 41

2.7 .3 SVM Não Lineares . . . . . . . . . . . . . . . . . . . . 43

2.8 REGRESSÃO POR MEIO DE MÁQUINAS DE SUPORTE VETORIAL . . . . . 45

2.9 AJUSTES DE CURVAS $\ldots \ldots \ldots \ldots \ldots \ldots$

2.10 PREVISÕES $\ldots \ldots \ldots \ldots$

2.11 ANÁLISE DOS MODELOS E MÉTODOS APRESENTADOS . . . . . . . . . . 47 
3 DEFINIÇÃO DO MODELO SVR-WAVELET . . . . . . . . . . . . . 51

3.1 MODELOS HíBRIDOS . . . . . . . . . . . . . . . . . . . 51

3.1 .1 O Modelo SVR-wavelet $\ldots \ldots \ldots \ldots$. . . . . . . . . . . . 51

3.1 .2 Técnicas de Denoising ou wavelet Shrinkage . . . . . . . . . . . . 51

3.2 PREDIÇÃO DE SÉRIES TEMPORAIS POR MEIO DO MODELO SVR-WAVELET HÍBRIDO . . . . . . . . . . . . . . . . . . . . 53

4 MÉTRICAS $\ldots \ldots \ldots \ldots \ldots \ldots \ldots$

4.1 TESTES DE NORMALIDADE $\ldots \ldots \ldots \ldots \ldots \ldots$

4.1 .1 Anderson-Darling . . . . . . . . . . . . . . . . . 56

4.1 .2 Cramer-von Mises . . . . . . . . . . . . . . . . . . . . . 57

4.1 .3 (Lilliefors) Kolmogorov-Smirnov . . . . . . . . . . . . . . . 57

4.1 .4 Pearson chi-square . . . . . . . . . . . . . . . . . . . . 58

4.1 .5 Shapiro-Wilk . . . . . . . . . . . . . . . . . . . . . 58

4.1 .6 Shapiro-Francia . . . . . . . . . . . . . . . . . . . . . . . . . . . . . 59

4.1 .7 Jarque-Bera $\ldots \ldots \ldots \ldots$

4.2 TESTES DE RAIZ UNITÁRIA $\ldots \ldots \ldots$

4.2 .1 Dickey-Fuller Aumentado . . . . . . . . . . . . . . . . . . . . . 59

4.2 .2 Phillips-Perron . . . . . . . . . . . . . . . . . . . 60

$4.2 .3 \quad \mathrm{KPSS} \ldots \ldots \ldots \ldots \ldots$

4.3 TESTES DE NÃO-LINEARIDADE $\ldots \ldots \ldots \ldots \ldots . \ldots \ldots$

4.3 .1 Terasvirta Neural Network . . . . . . . . . . . . . . . . . 61

4.3 .2 White Neural Network . . . . . . . . . . . . . . . . . 61

4.4 TESTES DE CORRELAÇÃO PELO GRÁFICO DE DISPERSÃO . . . . . . . . . 62

4.5 MSE, RMSE, MAE, MPE, MAPE, MASE, ACF1, AIC, AICC, BIC . . . . . . . 62

4.5 .1 MSE - Erro Quadrático Médio . . . . . . . . . . . . . . 62

4.5 .2 RMSE - Raiz Quadrada do Erro Quadrático Médio . . . . . . . . . 62

4.5 .3 MAE - Erro Absoluto Médio . . . . . . . . . . . . . . . 63

4.5 .4 MPE - Erro Porcentual Médio . . . . . . . . . . . . . . . 63

4.5 .5 MAPE - Erro Porcentual Absoluto Médio . . . . . . . . . . . . . . 63

4.5 .6 MASE - Erro Escalado Absoluto Médio . . . . . . . . . . . . 64

4.5 .7 ACF1 - Autocorrelação de Erros para uma Defasagem . . . . . . . 64

4.5 .8 AlC - Critério de Informação de Akaike . . . . . . . . . . 64

4.5 .9 AlCc - AlC de Segunda Ordem . . . . . . . . . . . . . 65

4.5.10 BIC - Critério de Informação Bayesiano . . . . . . . . . . . . 65

4.6 INTERPRETAÇÃO DOS P-VALUES . . . . . . . . . . . . . . . . . 65 
4.7 CRITÉRIOS PARA SELEÇÃO DO MELHOR MODELO . . . . . . . . . . 66

4.8 EXPOENTE DE HUST E A ANÁLISE R/S . . . . . . . . . . . . . . 66

4.8 .1 Método para Obtenção do Expoente de Hurst $\ldots \ldots \ldots$. . . . . 69

4.8 .2 Teste de Significância do Expoente de Hurst $\ldots \ldots \ldots$. . . . . 70

5 METODOLOGIA . . . . . . . . . . . . . . . . . . . . . 71

6 AMBIENTE DE PROGRAMACCÃO PARA ANÁLISE DE DADOS ESTATÍSTICOS E GRÁFICOS R . . . . . . . . . . . . . . . 73

6.1 USO DO MÉTODO SVM . . . . . . . . . . . . . . . . . . . . . . . . . 73

6.2 USO DO MÉTODO PREDICT . . . . . . . . . . . . . . . . . . . . . 74

6.3 USO DOS MÉTODOS WD E WR . . . . . . . . . . . . . . . . . 75

6.4 USO DOS MÉTODOS WAVMODWT E RECONSTRUCT . . . . . . . . . . . 76

6.5 USO DO MÉTODO THRESHOLD . . . . . . . . . . . . . . . . . . . 77

6.6 USO DOS MÉTODOS SARIMA E SARIMA.FOR . . . . . . . . . . . . . 77

6.7 USO DOS MÉTODOS ARFIMA E FORECAST . . . . . . . . . . . . . 78

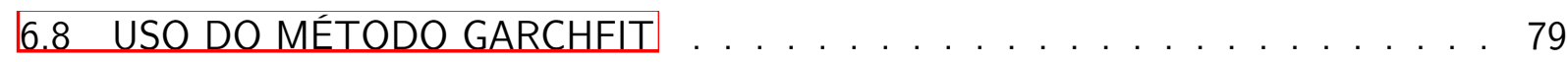

7 APLICACุÃO DO MODELO SVR-WAVELET . . . . . . . . . . . . . . . . 80

7.1 SELEÇÃO E CARACTERIZAÇÃO DAS SÉRIES TEMPORAIS DO TIPO FOREX. 80

7.2 TESTES DE NORMALIDADE, RAIZ UNITÁRIA E DE NÃO-LINEARIDADE . . . 83

7.3 TESTES DE CORRELAÇÃO PELO GRÁFICO DE DISPERSÃO . . . . . . . . . . 86

7.4 AJUSTES POR MEIO DO MODELO SVR TRADICIONAL COM KERNEL . . . . 87

7.4.1 Aplicação do Método SVR tradicional com Kernel em R . . . . . . . 88

7.4.2 Qualidade da Previsão por meio do Modelo SVR tradicional com Kernel 89

7.5 AJUSTES POR WAVELET: TRANSFORMADA E INVERSA . . . . . . . . . 990

7.5.1 Aplicação da Transformada wavelet e sua Inversa em R . . . . . . . . 90

7.5 .2 Qualidade da Transformada wavelet e sua Inversa . . . . . . . . . . 91

7.6 AJUSTES POR WAVELET MODWT UTILIZANDO A WAVELET HAAR . . . . . 91

7.6.1 Aplicação da wavelet MODWT e sua Inversa em R . . . . . . . . . 92

7.6 .2 Qualidade da wavelet MODWT e sua Inversa . . . . . . . . . . . . 92

7.7 A DECOMPOSIÇÃO EM WAVELETS . . . . . . . . . . . . . . . . . 93

7.8 MODELO HÍBRIDO SVR-WAVELET - AJUSTES E PREVISÃO DOS COMPO-

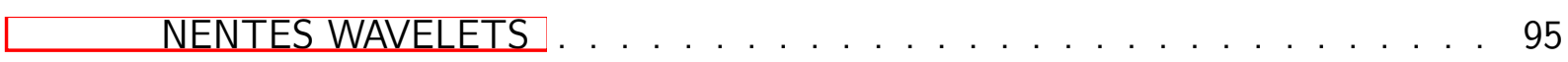

7.8.1 Qualidade da Previsão por meio do Modelo Híbrido SVR-wavelet . . . 96

7.9 AJUSTES POR MEIO DO MODELO ARIMA . . . . . . . . . . . . 96

7.9.1 Qualidade da Previsão por meio do Modelo ARIMA . . . . . . . . . . 96 
7.10 AJUSTES POR MEIO DO MODELO ARFIMA . . . . . . . . . . . . . . . 97

7.10 .1 Qualidade da Previsão por meio do Modelo ARFIMA . . . . . . . . 98

7.11 AJUSTES POR MEIO DO MODELO GARCH . . . . . . . . . . . . . . . . 98

7.11 .1 Qualidade da Previsão por meio do Modelo GARCH . . . . . . . . . . . 99

7.12 PARTE EXPERIMENTAL DO EXPOENTE DE HURST $\ldots \ldots$. . . . . . . 100

8 ANÁLISE DOS RESULTADOS $\ldots \ldots \ldots$

8.1 SELEÇ̃̃O DO MELHOR MODELO: ARIMA OU SVR-WAVELET HIBBRIDO ‥ 107

8.2 SELEÇÃO DO MELHOR MODELO: ARFIMA OU SVR-WAVELET HÍBRIDO. . . 108

8.3 SELEÇÃO DO MELHOR MODELO: GARCH OU SVR-WAVELET HíBRIDO . . . 109

8.4 SELEÇÃO DO MELHOR MODELO: SVR TRADICIONAL COM KERNEL OU

SVR-WAVELET HIBRIDO . . . . . . . . . . . . . . . . . . 110

8.5 CONCLUSÕES SOBRE A ADERÊNCCIA AO EXPOENTE DE HURST . . . . . . . 111

8.5.1 Resultados obtidos dos cálculos do Expoente de Hurst . . . . . . . 112

9 CONCLUSÃO . . . . . . . . . . . . . . . . . . . . . . . . . . 115

9.1 RESULTADOS OBTIDOS E CONTRIBUICCÕES ALCANÇADAS . . . . . . . . . . 115

9.2 SUGESTÕES PARA TRABALHOS FUTUROS . . . . . . . . . . . . . . 116

Referências . . . . . . . . . . . . . . . . . . . . . . . . . 118

Anexo A - Métodos em R - Cálculo dos Testes de Normalidade e de Raiz Unitária, Correção de Dados Discrepantes, Técnicas de Denoising ou Wa-

velet Shrinkage e Persistência de Plots . . . . . . . . . . . . . . . . 129

Anexo B - Métodos em R - Cálculo das Métricas MSE, RMSE, MAE, MPE, MAE, ACF1, AIC1, AICC1 e BIC1 . . . . . . . . . . . . . . 131

Anexo C - Métodos em R - Cálculo da Transformada wavelet e de sua Inversa 133 Anexo D - Métodos em R - Cálculo da Previsão dos Compontentes wavelet 134 Anexo E - Métodos em R - Cálculo da Previsão dos Modelos Tradicionais e Comparação com o Modelo Proposto . . . . . . . . . . . . . . . . . . 136

Anexo F - Métodos em R - Cálculo do Expoente de Hurst - Médias e Desvio Padrão . . . . . . . . . . . . . . . . . . . . . . . . . 137

Anexo G - Métodos em R - Cálculo do Expoente de Hurst por meio da

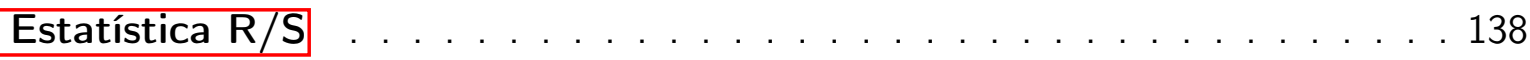


Anexo H - Métodos em R - Cálculo do Expoente de Hurst - Plot da Reta de Tendência e Cálculo do Valor de p-value . . . . . . . . . . . . . 139

Anexo I - Métodos em C\# - Cálculo da Divisão das Séries em Períodos de 15 min., 1 hora e 1 dia 140

Anexo J - Métodos em C\# - Cálculo da Divisão dos Dados nos Períodos de 15 min., 1 hora e 1 dia . . . . . . . . . . . . . . . . . . . . . . . . . . 143 


\section{Nomenclatura}

ACF1

Autocorrelation of Errors at Lag 1 - Autocorrelação de erros para uma defasagem

AIC

Akaike Information Criterion - Critério de Informação de Akaike

$\mathrm{AICc}$

Second-Order AIC - AIC de Segunda Ordem

ANN

Artificial Neural Networks - Redes Neurais Artificiais

ANOVA

Analysis of Variance - Análise de Variância

$\operatorname{AR}(\mathrm{p})$

Autoregressive Model ( $p$ ) Order - Modelo Autorregressivo de Ordem (p)

$\mathrm{ARCH}(\mathrm{m})$

Autoregressive Conditional Heteroskedasticity Model m Order - Modelo Autorregressivo Condicional com Heterocedasticidade de Ordem m

$\operatorname{ARFIMA}(\mathrm{p}, \mathrm{d}, \mathrm{q})$ Autoregressive Fractionally Integrated Moving Average Model (p, d, q) Order - Modelo Autorregressivo Fracionário Integrado de Médias Móveis de $\operatorname{Ordem}(p, d, q)$

$\operatorname{ARIMA}(p, d, q)$ Autoregressive Integrated Moving Average Model (p, d, q) Order - Modelo Autorregressivo Integrado de Médias Móveis de $\operatorname{Ordem}(p, d, q)$

$\operatorname{ARMA}(\mathrm{p}, \mathrm{q}) \quad$ Autoregressive Moving Average Model (p, q) Order - Modelo Autorregressivo e de Médias Móveis de Ordem ( $p, q)$

BIC Bayesian Information Criterion - Critério de Informação Bayesiano

BL Bayesian Learning - Aprendizagem Bayesiana

BLWF Best Localized wavelet Filter - Filtro wavelet de melhor localização no tempo e frequência na decomposição de um sinal

BPR Backpropagation with Bayesian Regularization - Algoritmo de Treinamento em Redes Neurais Artificiais

c.d.f. Empirical Cumulative Distribution Functions - Função de Densidade Cumulativa Empírica

Candlestick Nome de uma técnica de análise gráfica de mercado

CWT Continuous Wavelet Transform - Transformada de Wavelet Contínua

DL Deep Learning - Aprendizagem Profunda 
DWT Discrete Wavelet Transform - Transformada de Wavelet Discreta

EDOF Effective Degrees of Freedom - Graus de liberdade efetivos

ERM Empirical Risk Minimization - Princípio de Minimização do Risco Empírico

f.a.c. Autocorrelation Function - Função de Autocorrelação

FOREX Foreign Exchange Market - Mercado Global de Câmbio / Moedas

FX Foreign Exchange - Câmbio / Moedas

$\mathrm{GARCH}(\mathrm{m}, \mathrm{s}) \quad$ Generalized Autoregressive Conditional Heteroskedasticity Model (m, s) Order - Modelo Autorregressivo Condicional com Heterocedasticidade Generalizado de Ordem (m, s)

$\mathrm{H} \quad$ Hurst Exponent - Expoente ou Coeficiente de Hurst

HAAR Wavelet Haar proposta em 1909 por Alfréd Haar

HAC Heteroskedasticity and Autocorrelation Consistent - Heterocedasticidade e Autocorrelação Consistente

i.i.d. independent and identically distributed - independente e identicamente distribuído

ICWT Inverse Continuous Wavelet Transform - Transformada Wavelet Contínua Inversa

IDWT Inverse Discrete Wavelet Transform - Transformada de Wavelet Discreta Inversa

k-NN k-Nearest Neighbors Algorithm - Algoritmo k-Vizinhos mais Próximos

LR ～Logistic Regression - Regressão Logística

LS-SVM L Least Squares SVM - SVM por Mínimos Quadrados

MA(q) Moving Average Model (q) Order - Modelo de Médias Móveis de ordem (q)

MACD Moving Average Convergence-Divergence - Convergência/Divergência das Médias Móveis

MAE Mean Absolute Error - Erro Absoluto Médio

MAPE Mean Absolute Percentage Error - Erro Porcentual Absoluto Médio

MASE Mean Absolute Scaled Error - Erro Escalado Absoluto Médio

MLE Maximum-Likelihood Estimation - Estimativa por Máxima Verossimilhança 
MMSE Minimum Mean Square Error - Erro Quadrático Médio Mínimo

MODWT Maximal Overlap Discrete Wavelet Transform - Transformada Wavelet Discreta de Máxima Sobreposição

MPE

Mean Percentage Error - Erro Porcentual médio

MRA

Multiresolution Analysis - Análise Multirresolução

MSE

Mean Squared Error - Erro Quadrático Médio

NE

Normalized Error - Erro Normalizado

$\mathrm{R}$

Statistical Data Analysis R - Ambiente de Programação para Análise de Dados Estatísticos e Gráficos R

$\mathrm{R} / \mathrm{S}$

Estatistic Rescaled Range - Estatística de Reescalonamento - Estatística $\mathrm{R} / \mathrm{S}$

RBF Radial Basis Function Kernel - Função de Base Radial

RBFNN Radial Basis Function Neural Network - Modelos de Redes Neurais

RMSE $\quad$ Root Mean Square Error - Raiz Quadrada do Erro Quadrático Médio

RW Random Walk - Passeio Aleatório

SARIMA Seasonal ARIMA - Autoregressive Integrated Moving Average Model ( $p, d$,

q) Order - Modelo Sazonal Auto-Regressivo Integrado de Médias Móveis de Ordem ( $p, d, q)$

SBP Standard Backpropagation - Algoritmo de Treinamento em Redes Neurais Artificiais

SCG Scaled Conjugate Gradient - Algoritmo de Treinamento em Redes Neurais Artificiais

Sign Function - Função Sinal

SOM

Self Organizing Map - Mapas Auto-Organizáveis - Mapas de Kohonen

SRM

Structural Risk Minimization - Princípio de Minimização do Risco Estrutural

SV

Support Vectors - Vetores de Suporte

SVM

Support Vector Machines - Máquinas de Suporte Vetorial

SVR

Support Vector Regression - Regressão por meio de Máquinas de Suporte Vetorial 


\section{Notação}

$a_{t}$ : ruído branco no instante $t$.

$\alpha$ : Multiplicador de Lagrange

$b$ : Deslocamento (bias) de um hiperplano de separação para um SVR

$C$ : Constante de regularização de um SVR com margens suaves

$d$ : Distância entre os hiperplanos

$\delta, \kappa, d:$ Parâmetros da função Kernel Polinomial

$\delta, \kappa:$ Parâmetros da função Kernel Sigmoidal

$\xi$ : Variável de folga de um SVR

$\Im$ : Espaço de características

$\Gamma$ : Função gama

$\phi$ : vetor de coeficientes autorregressivos

$\Phi(x)$ : Função de mapeamento de um conjunto de dados não lineares

$H_{n}$ : Hiperplano $n, n=1 \ldots \infty$

$K\left(x_{i}, x_{j}\right)$ : Função Kernel de um SVR

$\mathcal{L}(w, b, \alpha)$ : Função Lagrangiana

$n$ : Número de amostras de treino em um conjunto

$n_{S V}:$ Número de vetores de suporte em um SVR

$\mu$ : média de um processo

$\Re^{n}$ : Espaço coordenado real ou espaço vetorial $n$-dimensional sobre $\Re$

$\sigma$ : Parâmetro da função Kernel Gaussiano (RBF)

$T$ : Conjunto de treinamento

$\theta$ : vetor de coeficientes de médias móveis

$w:$ Vetor de pesos em um SVR

$W_{t}$ : diferença de $X_{t}-X_{t-1}$, onde $X_{t}$ é um vetor contendo dados históricos de uma determinada série temporal

$x_{i}:$ Vetor de entrada no $i-$ ésimo item

$y_{i}$ : Vetor de rótulos no $i-e ́ s i m o$ item 


\section{INTRODUÇÃO}

Um dos objetivos da análise de séries temporais financeiras é a avaliação de riscos inerentes aos investimentos de carteiras de ativos financeiros (94). Este risco é normalmente medido em termos de variações de preços de ativos (95). Muitos dos estudos financeiros envolvem retornos de ativos. Campbell (18) fornece duas principais razões para a utilização dos retornos de ativos. Por um lado, para os investidores médios, o retorno de um ativo é um resumo completo e livre de escala da oportunidade de investimento. Por outro lado, as séries de retornos são mais fáceis de lidar do que séries de preços, porque os primeiros têm propriedades estatísticas mais atrativas como estacionariedade e ergodicidade.

Vários métodos e modelos têm sido desenvolvidos não somente para efetuar análise das oscilações de preços de ativos financeiros, mas também na previsão de dados de ativos financeiros e na seleção de dados relevantes e de comportamentos recorrentes (34) (104) (108), o que leva a argumentar sobre novas alternativas na predição de séries temporais financeiras.

Segundo Morettin (94), séries temporais podem ser modeladas por meio de processos do tipo Autorregressivo Fracionário Integrado de Médias Móveis (ARFIMA) ou ARIMA Fracionário. Este processo é considerado de memória longa pois sua função de autocorrelação (f.a.c.) decresce hiperbolicamente para zero. As séries temporais financeiras também podem ser modeladas por meio de processos Autorregressivos Condicionais com Heterocedasticidade $(\mathrm{ARCH})$ e por processos ARCH Generalizados (GARCH) (142).

O mercado de câmbio, Foreign Exchange Market (FOREX), também conhecido como Foreign Exchange (FX) ou Currency Market, é um mercado global para a negociação descentralizada de moedas (50). Em termos de volume de negociação, é de longe o maior mercado do mundo (125). Os principais participantes deste mercado são os maiores bancos internacionais e são eles os responsáveis pela definição dos valores relativos das diferentes moedas.

Com a globalização econômica as empresas que operam neste mercado estão inevitavelmente expostas aos riscos da flutuação das taxas de câmbio tanto positiva quanto negativamente. A capacidade de uma empresa para competir internacionalmente depende de sua capacidade de gerenciar de forma adequada e eficaz os riscos decorrentes de operações multinacionais. Consequentemente, a previsão deste tipo de ativo financeiro tem um significado importante para as empresas multinacionais e é um fator crucial para o sucesso dos gestores de fundos financeiros (1).

Muitos fatores econômicos, políticos e até mesmo psicológicos afetam os movimentos das taxas de câmbio, o que torna a previsão deste tipo de série uma tarefa não muito fácil tampouco trivial. As séries históricas deste tipo de ativo financeiro geralmente possuem características de não-estacionariedade o que faz com que métodos estatísticos tradicionais não sejam adequados 
nem eficientes na sua predição, sendo necessário a aplicação de técnicas e métodos de previsão mais avançados.

O objetivo deste trabalho é desenvolver um modelo de previsão adaptativo baseado em um sistema SVR-wavelet híbrido, que integra modelos de wavelets e Support Vector Regression (SVR), regressão por meio de Máquinas de Suporte Vetorial (SVM), para a previsão de séries financeiras. A utilização da Transformada de Wavelet Discreta (DWT), decompõe os dados de séries de ativos financeiros que posteriormente serão utilizados como variáveis de entrada do SVR para prever dados da série financeira. As séries ajustadas são comparadas com os modelos tradicionais Autorregressivo Integrado de Médias Móveis (ARIMA), Autorregressivo Fracionário Integrado de Médias Móveis (ARFIMA), Autorregressivo Condicional com Heterocedasticidade Generalizado (GARCH) e o modelo SVR tradicional com Kernel. Além disso, são efetuados testes de normalidade e de raiz unitária para distribuição não linear, tal como testes de correlação, para averiguar que as séries temporais FOREX são não-viciadas e apropriadas para a comprovação do modelo híbrido SVR-wavelet e comparação com modelos tradicionais. Suplementarmente o trabalho também verifica a aderência ao Expoente de Hurst, aplicando-se a estatística de Reescalonamento (R/S).

A análise por meio de wavelets possibilita efetuar a eliminação de ruídos contidos nas séries por meio de filtros e técnicas de denoising ou wavelet shrinkage para obtenção de uma versão mais limpa da série temporal original. Adiciona-se a isto o fato de que técnicas não-lineares como as Máquinas de Suporte Vetorial, Support Vector Machines (SVM), provaram ser eficazes na previsão de séries temporais (143) (124).

Este trabalho está organizado da seguinte forma: o Capítulo 2, Regressão e Métodos de Aprendizado de Máquinas, apresenta alguns dos mais relevantes métodos utilizados para regressão, como os modelos matemáticos tradicionalmente utilizados como regressores de séries temporais financeiras e de aprendizado de máquinas; também destaca conceitos relacionados à SVR, regressão por meio de Máquinas de Suporte Vetorial (SVM), e à Transformada wavelet; por fim, expressa uma análise crítica e o posicionamento deste trabalho frente às diversas iniciativas de pesquisa acadêmicas que vêm sendo desenvolvidas e realizadas sobre a utilização de métodos de aprendizado de máquinas na regressão de séries temporais. O Capítulo 3, Definição do Modelo SVR-wavelet, retrata os modelos híbridos (em particular o modelo de previsão por meio do sistema SVR-wavelet) que integram modelos wavelets e SVR e traz uma proposição para a definição matemática do modelo SVR-wavelet híbrido. O Capitulo 4, Métricas, discorre sobre uma variedade de testes estatísticos (como os de normalidade e de raiz unitária), com o propósito de confirmar que as séries temporais FOREX são apropriadas para a comprovação do modelo híbrido SVR-wavelet; também são apresentadas métricas da precisão da previsão do modelo ajustado; é discutido o Expoente de Hurst, por intermédio da estatís- 
tica $R / S$, como uma métrica que permite verificar a existência da dinâmica fractal em séries temporais FOREX. O Capítulo 5, Metodologia, apresenta o método para previsão de séries temporais financeiras do tipo FOREX, baseando-se no modelo SVR-wavelet híbrido discutido no Capítulo 3. O Capítulo 6, Ambiente de Programação para Análise de Dados Estatísticos e Gráficos $R$, discorre sobe o ambiente de programação para análise de dados estatísticos e gráficos $R$ e os métodos estatísticos utilizados pelo trabalho. O Capítulo 7, Aplicação do modelo SVR-wavelet, efetua o ajuste e a previsão dos dados das séries temporais financeiras do tipo FOREX por meio do modelo SVR-wavelet híbrido, apresentando uma avaliação dos resultados do desempenho do modelo proposto e sua comparação com modelos tradicionais ARFIMA, ARIMA, GARCH e o modelo SVR tradicional com Kernel; também discorre sobre as séries temporais FOREX, Foreign Exchange Market, sua evolução e suas características, sob as quais são aplicados testes de normalidade e de raiz unitária, assim como testes de correlação, para se aferir a qualidade das séries FOREX; por fim, apresenta a parte experimental do Expoente de Hurst, por meio da estatística R/S. O Capítulo 8, Análise dos Resultados, desenvolve uma análise sobre a seleção do melhor modelo, comparando o modelo SVR-wavelet proposto com modelos tradicionais ARFIMA, ARIMA, GARCH e o modelo SVR tradicional com Kernel, traçando as conclusões sobre a aderência do Expoente de Hurst através da estatística R/S. O Capítulo 9, Conclusão, é dedicado à análise geral, às contribuições do trabalho, bem como sugestões para futuros trabalhos. 


\section{REGRESSÃO E MÉTODOS DE APRENDIZADO DE MÁQUINAS}

A utilização de negociações eletrônicas por meio de algoritmos de negociação, o acompanhamento do mercado através da análise técnica e da análise fundamentalista e a utilização de modelos matemáticos por intermédio de métodos de aprendizado de máquinas na regressão de séries temporais são exemplos de iniciativas de pesquisas sobre a maximização de retornos financeiros.

\subsection{ALGORITHMIC TRADING}

A utilização de negociações eletrônicas por meio de algoritmos de negociação se procede por meio dos Algorithmic Trading, muitas vezes denominados por Algo Trading ou Blackbox Trading. Trata-se de sistemas de negociação que são dependentes de formulação matemática e de sistemas de computadores de alta velocidade para determinar estratégias de negociação (71) (74).

Estas estratégias usam plataformas eletrônicas que registram ordens de negociação através de um algoritmo que executa instruções de negociação pré-programados sobre uma variedade de variáveis como tempo, preço e volume (73).

Existem algumas características na utilização de negociações eletrônicas por intermédio dos Algo Trading, entre as quais se destacam (41): minimizar as emoções, preservar a disciplina, coerência, maior velocidade na entrada de ordens, controle de falhas mecânicas e capacidade de back-test.

\subsection{ANÁLISE TÉCNICA E ANÁLISE FUNDAMENTALISTA}

A perspectiva de acompanhamento do mercado financeiro por meio da análise técnica, empregando análise gráfica de ativos financeiros, bem como por meio da análise fundamentalista de ativos financeiros é outra motivação de pesquisa sobre a maximização de retornos financeiros.

\subsubsection{Análise Técnica}

A análise técnica é composta por um conjunto de ferramentas que o investidor dispõe para auxílio na decisão de investimentos com base em gráficos e indicadores técnicos. Ela é dividida em dois grupos de estudo: análise gráfica e análise técnica. Os gráficos do comportamento de preços ao longo do tempo constituem-se nas principais ferramentas de análise gráfica (52). 
Os padrões gráficos são figuras que surgem com base na força de movimentos entre os compradores e os vendedores que, ao longo do tempo, delineiam tendências (13). Pelo padrão, é possível prever o comportamento subsequente de preços admitindo a existência da repetição de figuras e assim concretizar a possibilidade de previsão com base nos gráficos. Outras ferramentas como os Candlestick, gráfico de barras e os números de Fibonacci, utilizam gráficos de preço e de volume para prever o comportamento de ativos financeiros (13).

Esta análise consiste na realização de cálculos utilizando-se das cotações e dos volumes negociados do fechamento de pregões anteriores para projetar possíveis tendências futuras. Assim, a proposta da análise técnica é entender como os ativos financeiros realizam ou não movimentos, visando portanto capturar as principais reversões de mercado (52).

Os principais instrumentos produzidos a partir de ferramentas matemáticas e estatísticas são: Médias Móveis, Estocástico, Momentum, Moving Average Convergence-Divergence (MACD) (103).

Existem algumas características na utilização da análise técnica entre as quais se destacam (67): as notícias refletem os preços, identificação de tendências, indicação de recomendações de entrada e saída, sinais contraditórios entre outros indicadores técnicos e precisão da previsão que não é $100 \%$.

\subsubsection{Análise Fundamentalista}

A análise fundamentalista adota a hipótese da existência de um valor intrínseco para cada ação, com base nos resultados apurados pelas empresas emitentes. O estudo dessa análise está baseado no desenvolvimento econômico das empresas e no processamento de avaliações e comparações setoriais, bursáteis e conjunturais (128).

Os principais subsídios desse critério de análise são os demostrativos financeiros das empresas e os diversos dados e informações referentes aos setores de atividade econômica, ao mercado acionário e à conjuntura econômica (101).

Existem algumas características da análise fundamentalista entre as quais se destacam (27): conhecimentos específicos requeridos, prazo de investimento, movimento do mercado e a fama das empresas.

\subsection{MODELOS MATEMÁTICOS}

Da mesma forma que os métodos apresentados para acompanhamento do mercado através da análise técnica e da análise fundamentalista os modelos Autorregressivos $A R(p)$, de Médias Móveis $M A(q)$, Autorregressivos e de Médias Móveis $\operatorname{ARMA}(p, q)$, Autorregressivos 
Integrados de Médias Móveis $\operatorname{ARIMA}(p, d, q)$, Autorregressivos Fracionários Integrados de Médias Móveis $\operatorname{ARFIMA}(p, d, q)$, Autorregressivos Condicionais com Heterocedasticidade $A R C H(m)$ e os modelos Autorregressivos Condicionais com Heterocedasticidade Generalizados $\operatorname{GARCH}(\mathrm{m}, \mathrm{s})$ são muito utilizados em economia com a finalidade de se elaborar modelos de previsão (94).

Segundo Morettin e Toloi (94) há duas classes de modelos envolvendo séries temporais, de acordo com os números de parâmetros envolvidos: modelos paramétricos e não-paramétricos.

Para estes autores (94), na classe de modelos paramétricos, a análise é feita no domínio do tempo. Os modelos mais frequentemente usados são os modelos de regressão: modelos de memória longa - Autorregressivos Fracionários Integrados de Médias Móveis (ARFIMA), os modelos Autorregressivos Integrados de Médias Móveis (ARIMA) e os modelos Autorregressivos Condicionais com Heterocedasticidade Generalizados (GARCH).

\subsubsection{Modelos Autorregressivos}

Segundo Morettin (94), o modelo autorregressivo de ordem $p, A R(p)$, é dado por:

$$
X_{t}-\mu=\phi_{1}\left(X_{t-1}-\mu\right)+\cdots+\phi_{p}\left(X_{t-p}-\mu\right)+a_{t}
$$

em que $\left\{X_{t}, t \in \mathbb{Z}\right\}$ é um processo estocástico estacionário, $X_{t} \sim A R(p), \mu, \phi_{1}, \ldots, \phi_{p}$ são parâmetros reais, $\phi=\left(\phi_{1}, \ldots, \phi_{p}\right)$ é o vetor de coeficientes autorregressivos e $a_{t} \sim R B\left(0, \sigma^{2}\right)$ é o ruído branco no instante $t$.

Segue-se que $\mu=\boldsymbol{E}\left(X_{t}\right)=\left[\phi_{0} /\left(1-\phi_{1}-\phi_{2}-\cdots-\phi_{p}\right)\right]$ e o processo se escreve na forma:

$$
X_{t}=\phi_{0}+\phi_{1} X_{t-1}+\cdots+\phi_{p} X_{t-p}+a_{t}
$$

Assim, o valor de $X_{t}$ depende dos $p$ valores anteriores da série e do ruído branco no instante t. O caso mais simples, $A R(1)$, ocorre quando $p=1$ e $\mu=0: X_{t}=\phi_{1} X_{t-1}+a_{t}$, onde há a dependência de $X_{t-1}$ e de $a_{t}$.

De modo mais conciso, com o operador retroativo, $B^{s} X_{t}=X_{t-s}, s \geq 1$, com $s=1$, o modelo $A R(p)$, apresentado pela equação 1 , com $\mu=0$, pode ser reescrito como $\phi(B) X_{t}=$ $a_{t}$. 


\subsubsection{Modelos de Médias Móveis}

Um processo de médias móveis de ordem $q, M A(q)$, é também um processo estocástico estacionário, em que $\left\{X_{t}, t \in \mathbb{Z}\right\}$ e $X_{t} \sim M A(q)$, satisfazendo a equação de diferenças (94):

$$
X_{t}=\mu+a_{t}-\theta_{1} a_{t-1}-\cdots-\theta_{q} a_{t-q}
$$

em que $\mu, \theta_{1}, \ldots, \theta_{q}$ são constantes reais, $\theta=\left(\theta_{1}, \ldots, \theta_{q}\right)$ é o vetor de coeficientes de médias móveis e $a_{t} \sim R B\left(0, \sigma^{2}\right)$ é o ruído branco no instante $t$. O caso mais simples, $M A(1)$, ocorre quando $q=1$ e $\mu=0: X_{t}=a_{t}-\theta_{1} a_{t-1}$. O modelo $M A(q)$, expresso pela equação 3 , com $\mu=0$, também pode ser reescrito de modo mais sintético, por meio do operador retroativo, $B^{s} X_{t}=X_{t-s}, s \geq 1$, com $s=1$, como $X_{t}=\theta(B) a_{t}$.

\subsubsection{Modelos Autorregressivos e de Médias Móveis}

Os modelos autorregressivos são muito utilizados em economia, onde é natural pensar o valor de alguma variável no instante $t$ como função de valores defasados em relação ao instante $t$, com a finalidade de se elaborar um modelo de previsão (94).

Para modelar estes processos com um número pequeno de parâmetros, uma solução adequada é aquela que inclui termos autorregressivos e de médias móveis. Assim, o modelo $A R M A(p, q)$ é formulado como:

$$
X_{t}-\mu=\phi_{1}\left(X_{t-1}-\mu\right)+\cdots+\phi_{p}\left(X_{t-p}-\mu\right)+a_{t}-\theta_{1} a_{t-1}-\cdots-\theta_{q} a_{t-q}
$$

em que $\phi=\left(\phi_{1}, \ldots, \phi_{p}\right)$ é o vetor de coeficientes autorregressivos, $\theta=\left(\theta_{1}, \ldots, \theta_{q}\right)$ é o vetor de coeficientes de médias móveis e $a_{t} \sim R B\left(0, \sigma^{2}\right)$ é o ruído branco no instante $t$ (94). Um modelo frequentemente utilizado é o $A R M A(1,1)$, com $\mu=0: X_{t}=\phi_{1} X_{t-1}+a_{t}-\theta_{1} a_{t-1}$. O modelo $A R M A(p, q)$, indicado pela equação 4 , com $\mu=0$, pode ser também reescrito sucintamente, através do operador retroativo, $B^{s} X_{t}=X_{t-s}, s \geq 1$, com $s=1$, como $\phi(B) X_{t}=\theta(B) a_{t}$.

\subsubsection{Modelos Autorregressivos Integrados de Médias Móveis}

Morettin (94) mostra que um processo $\left\{X_{t}, t \in \mathbb{Z}\right\}$ segue um modelo $A R I M A(p, d, q)$ se $\triangle^{d} X_{t}$ seguir um modelo $A R M A(p, q)$. Assim o modelo autorregressivo integrado de média 
móvel (ARIMA) de ordem $p, d, q$, denotado por $\operatorname{ARIMA}(p, d, q)$ é definido como:

$$
\phi(B) \triangle^{d} X_{t}=\theta_{0}+\theta(B) a_{t}
$$

sendo $p$ e $q$ respectivamente as ordens de $\phi(B)$ e $\theta(B)$ (94). Este modelo supõe que a $d$-ésima diferença da série $X_{t}$ pode ser representada por um modelo $A R M A$.

De modo similar, $\phi(B) W(t)=\theta_{0}+\theta(B) a_{t}$, com $W(t)=\triangle^{d} X_{t}$. Verifica-se que $W(t)=$ $\triangle^{d} X_{t} \Longleftrightarrow X_{t}=S^{d} W(t)$, em que $S$ é o operador soma ou integrador (94).

Casos particulares do modelo $\operatorname{ARIMA}(p, d, q)$ são:

- $\operatorname{ARIMA}(p, 0,0)=A R(p)$;

- $\operatorname{ARIMA}(0,0, q)=M A(q) ; \mathrm{e}$

- $\operatorname{ARIMA}(p, 0, q)=A R M A(p, q)$.

\subsubsection{Modelos Autorregressivos Fracionários Integrados de Médias Móveis}

Um processo de memória longa é um processo cuja função de autocorrelação (f.a.c.), $\rho_{j}$, decresce hiperbolicamente.

Procurando respeitar as características de uma série de memória longa, no decorrer do tempo ocorreu a definição de dois modelos importantes. No primeiro, foi introduzido o ruído gaussiano fracionário por Mandelbrot e Van Ness em 1968 (84). Mais tarde, em 1980, Granger e Joyeux (51) e posteriormente em 1981 Hosking (61) introduziram o modelo Autorregressivo Fracionário Integrado de Médias Móveis (ARFIMA) ou ARIMA Fracionário, que é uma generalização do modelo ARIMA.

Assim uma série temporal $\left\{X_{t}\right\}$ é um processo autorregressivo fracionário integrado de médias móveis, ou $\operatorname{ARFIMA}(p, d, q)$ com $d \in(-1 / 2,1 / 2)$, se $\left\{X_{t}\right\}$ for estacionário, conforme modelo (94):

$$
\phi(B)(1-B)^{d} X_{t}=\theta(B) a_{t}
$$

em que $a_{t} \sim R B\left(0, \sigma^{2}\right)$ é o ruído branco no instante $t$ e $\phi(B)$ e $\theta(B)$ são polinômios em $B$, respectivamente de graus $p$ e $q$.

O processo $A R F I M A(p, d, q)$ é estacionário se $d<1 / 2$ e todas as raízes de $\phi(B)=0$ estiverem fora do círculo unitário e invertível se $d>-1 / 2$ e todas as raízes de $\theta(B)=0$ também estiverem fora do círculo unitário (61), como nos processos estacionários e invertíveis (94): 
- $\operatorname{ARFIMA}(1, d, 0)$, equacionado como $(1-B)^{d}(1-\phi B) X_{t}=a_{t}$, se $|d|<1 / 2$ e $|\phi|<1$; e

- $\operatorname{ARFIMA}(0, d, 1)$, equacionado como $(1-B)^{d} X_{t}=(1-\theta B) a_{t}$, se $|\theta|<1$ e $|d|<$ $1 / 2$. Esta última formulação é definida como uma média móvel de primeira order de um ruído branco fracionário.

O caso mais simples é o ruído branco fracionário, isto é, $\operatorname{ARFIMA}(0, d, 0)$, formulado como $(1-B) X_{t}=a_{t}, a_{t} \sim R B\left(0, \sigma^{2}\right)$.

\subsubsection{Modelos Autorregressivos Condicionais com Heterocedasticidade}

Os modelos autorregressivos condicionais com Heterocedasticidade de ordem $m, A R C H(m)$, são modelos econométricos para modelar a volatilidade de retornos de ativos (142), podendo ser formulado como:

$$
a_{t}=\sigma_{t} \epsilon_{t}, \quad \sigma_{t}^{2}=\alpha_{0}+\alpha_{1} a_{t-1}^{2}+\cdots+\alpha_{m} a_{t-m}^{2}
$$

em que $\left\{\epsilon_{t}\right\}$ é uma sequência de variáveis aleatórias i.i.d. com média zero e variância 1 , $\alpha_{0}>0$ e $\alpha_{1} \geq 0$ para $i>0$. A equação 7 também é equacionada como $a_{t}=\sqrt{h_{t}} \epsilon_{t}$. O caso mais simples ocorre quando $m=1, A R C H(1)$, em que $\alpha_{0}>0$ e $\alpha_{1} \geq 0$ : $a_{t}=\sigma_{t} \epsilon_{t}, \sigma_{t}^{2}=$ $\alpha_{0}+\alpha_{1} a_{t-1}^{2}$.

\subsubsection{Modelos ARCH Generalizados}

O modelo GARCH, Autorregressivo Condicional com Heterocedasticidade Generalizado, ARCH Generalizado de ordem $(m, s)$ ou simplesmente $\operatorname{GARCH}(m, s)$, é outro modelo tradicionalmente usado como regressor de séries temporais financeiras, sendo equacionado como (142):

$$
a_{t}=\sigma_{t} \epsilon_{t}, \quad \sigma_{t}^{2}=\alpha_{0}+\sum_{i=1}^{m} \alpha_{i} a_{t-i}^{2}+\sum_{j=1}^{s} \beta_{j} \sigma_{t-j}^{2}
$$

em que $\left\{\epsilon_{t}\right\}$ é uma sequência de variáveis aleatórias i.i.d. com média 0 e variância $1, \alpha_{0}>0$, $\alpha_{i} \geq 0, \beta_{j} \geq 0$ e $\sum_{i=1}^{\max (m, s)}\left(\alpha_{i}+\beta_{j}\right)<1$ (142).

Um modelo frequentemente aplicado à séries financeiras é o modelo $G A R C H(1,1)$ :

$$
a_{t}=\sigma_{t} \epsilon_{t}, \quad \sigma_{t}^{2}=\alpha_{0}+\beta_{1} \sigma_{t-1}^{2}+\alpha_{1} a_{t-1}^{2}
$$




\subsection{MODELOS FRACTAIS}

Baseada na hipótese da existência de um Mercado Fractal, essa linha de pesquisa propõe a hipótese de que os Mercados Eficientes possuem algumas exceções ao pressuposto da normalidade (112). Osborne (104) havia constatado uma anomalia quando tentou plotar um gráfico da função densidade de probabilidade da distribuição dos retornos. A distribuição apresentava curtose mais elevada que a distribuição normal, leptocúrticas, ou de cauda pesada. Desde Cootner (24) já era bastante conhecido a existência de um comportamento de caudas pesadas na distribuição de mudanças de preços.

Estudos mostram evidências no sentido de que os retornos de títulos nos mercados de capitais não são normalmente distribuídos (106) (36) (126). Assim, se os retornos não forem normalmente distribuídos, boa parte da análise estatística, aquela que vale de coeficientes de correlação, fica bastante comprometida podendo levar a resultados equivocados, sugerindo também que a idéia da ocorrência de passeio aleatório nos preços das ações é equivocada.

A Teoria Fractal começou a se impor como um contraponto às hipóteses criadas pela teoria financeira mais conservadoras pelo fato de oferecer um prisma mais realista sobre 0 funcionamento do Mercado Financeiro (83).

Chamados de auto similares, os fractais tem estruturas menos elaboradas, cujas mudanças de escala se manifestam de maneira proporcional em toda estrutura. Os fractais mais parecidos com o comportamento de preços são os auto afins, pois mudam de escalas em direções diversas e, conforme constatado por alguns autores (69) (133) (5) (119) (109) (32), os mercados financeiros globais apresentam simetria de escala, com características fractais.

\subsection{TRANSFORMADA WAVELET}

A análise de wavelets é uma técnica matemática muito útil para análise numérica e manipulação de sinais discretos uni ou multidimensionais. As wavelets ampliam intervalos de dados, separando-os em diferentes componentes de frequência e escala, permitindo a análise de cada componente em sua escala correspondente. A idéia de aproximação, mediante a superposição de funções, tem sua origem nos trabalhos de Joseph Fourier que, no século XIX descobriu que poderia utilizar senos e cossenos para representar outras funções (91).

A novidade em relação a Fourier é que a base das funções de Fourier são dependentes da frequência mas não do tempo, ou seja, pequenas alterações no domínio da frequência produzem alterações em todo o domínio do tempo. As wavelets são dependentes de ambos os domínios, da frequência, via dilatação, e do tempo, via translação, o que é uma vantagem em diversos casos (140). Esta é a razão pela qual elas podem distinguir as características locais 
de um sinal em diferentes escalas e, por translações, elas cobrem toda a região na qual o sinal é estudado (37) (72).

Por causa de suas propriedades as wavelets são usadas em análise funcional, em estudo de propriedades multi-fractais, em reconhecimento de padrões, em compressão de imagens e de sons entre outros (8).

Assim, tanto na análise de Fourier, quanto na análise de wavelets, a idéia é aproximar uma função pela combinação linear de senos e cossenos ou wavelets, respectivamente (93).

Entretanto, na análise de Fourier, a função periódica tem período $2 \pi$, de quadrado integrável, $L^{2}(0,2 \pi)$, gerada pela superposição de exponenciais complexas, $\omega_{n}(t)=e^{i n t}$, $n=0, \pm 1, \ldots$, obtidas por dilatação da função $\omega(t)=e^{i t}$. Este conceito foi estendido para $L^{2}(\Re)$, em outras palavras, esse espaço é criado a partir de uma única função $\Psi$, chamada de wavelet mãe, gerado por dilatações e translações de $\Psi$, produzindo uma família de funções $\Psi_{a, b}(145)$ :

$$
\Psi_{a, b}(t)=\frac{1}{\sqrt{|a|}} \Psi\left(\frac{t-b}{a}\right)
$$

em que $a, b \in R$ e $a \neq 0$.

Quando se trata de sinais discretos, frequentemente usam-se valores particulares para $a$ e $b, a=2^{j}$ e $b=k 2^{j}$, com $k, j \in Z$, conforme:

$$
\Psi_{j, k}(t)=2^{-j / 2} \Psi\left(2^{-j} t-k\right)
$$

em que $j, k \in Z$, sendo que $\Psi_{j, k}(t)$ é obtida de $\Psi(t)$ por dilatação de $2^{-j}$ e uma translação $k 2^{j}$.

As funções $\left\{\Psi_{j, k}(t), j, k \in Z\right\}$ constituem uma base que obrigatoriamente não precisa ser ortogonal, todavia, empregando-se bases ortogonais é possível a reconstrução congênere do sinal original por meio dos coeficientes da transformada (93).

Ponderando uma base ortonormal gerada por $\Psi, \Psi_{j, k}(t)=2^{-j / 2} \Psi\left(2^{-j} t-k\right)$, com $j, k \in Z$, de maneira que qualquer $f(t) \in L^{2}(\Re)$ é formulado como (22):

$$
f(t)=\sum_{j=-\infty}^{\infty} \sum_{k=-\infty}^{\infty} c_{j, k} \Psi_{j, k}(t)
$$

em que $f(t)$ é uma série de wavelets com coeficientes denotado por:

$$
c_{j, k}=\left\langle f, \Psi_{j, k}\right\rangle=\int_{-\infty}^{\infty} f(t) \Psi_{j, k}(t) d t
$$

Existem diferentes tipos de wavelets ortogonais e algumas possuem atributos mais apropriadas para determinadas aplicações. Um modo para geração de wavelets ortogonais é pela 
função escala ou wavelet pai formulado como (80):

$$
\phi(t)=\sqrt{2} \sum_{k} h_{k} \phi(2 t-k)
$$

Essa formulação possibilita gerar uma família ortogonal de $L^{2}(\Re)$ conforme (93) (31):

$$
\phi_{j, k}(t)=2^{-j / 2} \phi\left(2^{-j} t-k\right)
$$

em que $j, k \in Z$. Então, nessas condições, $\Psi$ pode computado por:

$$
\Psi(t)=\sqrt{2} \sum_{k} g_{k} \phi(2 t-k)
$$

em que $g_{k}=(-1)^{k} h_{1-k}$. Chamadas equações de dilatação (93) (31) $h_{k}$ e $g_{k}$ são coeficientes de filtros passa-baixa e passa-alta usados para calcular a Transformada de Wavelet Discreta (DWT), conforme:

$$
h_{k}=\sqrt{2} \int_{-\infty}^{\infty} \phi(t) \phi(2 t-k) d t
$$

e

$$
g_{k}=\sqrt{2} \int_{-\infty}^{\infty} \Psi(t) \phi(2 t-k) d t
$$

Com isso, haja vista o sistema ortonormal $\left\{\phi_{j, k}(t), \Psi_{j, k}(t), j, k \in Z\right\}$, pode-se formular $f(t) \in L^{2}(\Re)$ como:

$$
f(t)=\sum_{k} c_{J, k} \phi_{J, k}(t)+\sum_{j \leq J} \sum_{k} d_{j, k} \Psi_{j, k}(t)
$$

em que:

$$
c_{J, k}=\left\langle f(t), \phi_{J, k}(t)\right\rangle=\int_{-\infty}^{\infty} f(t) \phi_{J, k}(t) d t
$$

e

$$
d_{j, k}=\left\langle f(t), \Psi_{j, k}(t)\right\rangle=\int_{-\infty}^{\infty} f(t) \Psi_{j, k}(t) d t
$$

\subsubsection{Análise Multirresolução (MRA)}

Introduzida por Mallat (80) a Análise Multirresolução (MRA) constitui-se de uma sequência de subespaços fechados $V_{j}$, onde $V_{j} \subset L^{2}(\Re)$, em que cada $V_{j}$ usa diferentes resoluções, daí o nome Análise Multirresolução, atendendo (93) (31) (65): 
- MR1) $\cdots V_{2} \subset V_{1} \subset V_{0} \subset V_{-1} \subset V_{-2} \subset \cdots$;

- MR2) $\overline{\bigcup_{j \in Z} V_{j}}=L^{2}(\Re)$;

- MR3) $\overline{\bigcap_{j \in Z} V_{j}}=\lim _{j \rightarrow+\infty} V_{j}=\{0\}$;

- MR4) $f(t) \in V_{j} \subset L^{2}(\Re) \Leftrightarrow f(2 t) \in V_{j-1}, j \in Z$ (Invariância em escala)

- MR5) $f(t) \in V_{j} \Leftrightarrow f\left(t-2^{j} k\right) \in V_{j},(j, k) \in Z^{2}$ (Invariância em translações)

- MR6) Existência de uma função $\phi$, função escala, em que $\left\{\phi_{j, k} ; j \in Z\right\}$ é uma base ortonormal em $V j$ onde $\phi_{j, k}(t)$ é formulada pela equação 15.

Considerando uma série de tempo $f(t) \in L^{2}(\Re)$, da qual se quer obter aproximações em vários níveis de resolução, cada subespaço $V_{j}$ é formado por aproximações de funções, em que a melhor aproximação é alcançada tendo em vista a projeção ortogonal $\left(P_{j}\right)$ de $f$ sobre cada $V_{j}, \operatorname{assim}(93)$ :

$$
\forall g(t) \in V_{j},\|g(t)-f(t)\| \geq\left\|P_{j}(t)-f(t)\right\|
$$

Afirmar que $V_{j} \subset V_{j-1}$ representa dizer que ao passar do nível de resolução $j$, escala $2^{j}$, para o nível $j-1$, ganha-se informação ou adiciona-se detalhes.

À medida que a resolução $2^{-j}$ aumenta, $j \rightarrow-\infty$, a função de aproximação converge para a função original, $P_{j} f \rightarrow f$, ou melhor, contém mais informações sobre $f$ e obtém-se (MR2) (80). De outra forma, quando a resolução $2^{-j}$ decresce para zero, (MR3) provoca a perda de todas as informações de $f$.

A propriedade (MR4) está concernente com a (MR1), já que à medida que $j$ decresce, refinando a escala, a resolução em frequência aumenta. Consequentemente, detalhes que aparecem em uma escala $2^{j}$ também devem estar presentes na escala $2^{j-1}$ (77). A propriedade (MR5) sugere que $V_{j}$ é invariante a qualquer translação proporcional a escala $2^{j}$.

A informação que é perdida quando se passa de $V_{j-1}$ para $V_{j}$ pode ser caracterizada pelo subespaço $W_{j}$, complemento ortogonal de $V_{j}$ em $V_{j-1}$, conforme:

$$
V_{j} \oplus W_{j}=V_{j-1}, W_{j} \perp V_{j}
$$

onde $\oplus$ indica a soma direta. Sucede que $V_{j}=\oplus_{j+1}^{j=+\infty} W_{j}$.

Haja vista $\Psi(t)$, determinada pela formulação 16 , em que $\left\{\Psi_{j, k} ; k \in Z\right\}$ forma uma base ortogonal para $W_{j}$, de maneira que, $\forall f \in L^{2}(\Re)$, pode-se formular $f$ como na equação 19 .

A Análise Multirresolução (MRA) conduz a um método hierárquico rápido para o cálculo dos coeficientes wavelet de uma dada função. 


\subsubsection{Transformada de Wavelet Discreta}

Para a formulação da Transformada de Wavelet Discreta (DWT) e com o propósito de se trabalhar com séries temporais, assume-se $\Psi_{a, b}$ como definida pela equação 11 . De acordo com Von Sachs, Nason e Kroisandt (146), e Morettin (93), os coeficientes wavelets da DWT podem ser representados por:

$$
d_{j, k, n}=\sum_{t=0}^{n-1} x_{t} \Psi_{j, k}(t)
$$

em que $j=0,1,2, \ldots, J, k=0,1,2, \ldots, 2^{j}, X=\left(x_{0}, x_{1}, \ldots, x_{n-1}\right)^{T}, J$ inteiro representando a escala mais grossa ou suave, perfazendo $n$ coeficientes $d$, e $n=2^{J}$ observações de um processo estocástico ou uma série temporal.

Considerando que uma série de tempo $f(t) \in L^{2}(\Re)$ possa ser representada em relação a uma base de wavelets como na equação $19, f(t)=\sum_{k} c_{J, k} \phi_{J, k}(t)+\sum_{j \leq J} \sum_{k} d_{j, k} \Psi_{j, k}(t)$, pode-se calcular os coeficientes $d_{j, k}$ e $c_{J, k}$ valendo-se da equação da função escala $\phi$, equação 14 , e da definição de wavelet $\Psi$, equação 16 , conforme:

$$
\begin{gathered}
d_{j, k}=\left\langle f, \Psi_{j, k}\right\rangle_{L^{2}}=\sum_{n \in Z} g_{n}\left\langle f, \phi_{j-1,2 k+n}\right\rangle, \\
d_{j, k}=\sum_{n \in Z} \overline{g_{n-2 k}} c_{j-1, n}, \\
c_{j, k}=\left\langle f, \phi_{j, k}\right\rangle_{L^{2}}=\sum_{n \in Z} h_{n}\left\langle f, \phi_{j-1,2 k+n}\right\rangle
\end{gathered}
$$

e

$$
c_{j, k}=\sum_{n \in Z} \overline{h_{n-2 k}} c_{j-1, n}
$$

sendo que $h_{k}$ e $g_{k}$ são formulados respectivamente pelas equações 17 e 18 .

\subsubsection{Transformada Wavelet Discreta de Máxima Sobreposição}

A Transformada wavelet Discreta de Máxima Sobreposição (MODWT), é uma versão modificada da Transformada de Wavelet Discreta (DWT). A MODWT permite a utilização da Análise Multirresolução (MRA). Usa-se a MODWT para se resolver a limitação da Transformada de Wavelet Discreta (DWT), que requer $N=2^{J}$ em que $J$ é um inteiro positivo (157). 
A definição da MODWT é obtida diretamente da DWT. Seja $\left\{h_{j, k}\right\}$ o filtro de wavelet DWT e $\left\{g_{j, k}\right\}$ o filtro de escala, sendo $k=1, \ldots, L$ o comprimento do filtro com $j$ níveis de decomposição. O filtro de wavelet MODWT $\left\{\tilde{h}_{j, k}\right\}$ e o filtro de escala MODWT $\left\{\tilde{g}_{j, k}\right\}$ são definidos como $\tilde{h}_{j, k}=h_{j, k} / 2^{j / 2}$ e $\tilde{g}_{j, k}=g_{j, k} / 2^{j / 2}$.

Então, os coeficientes da wavelet MODWT de nível $j$ são definidos como a convolução da série de tempo $\left\{X_{t}\right\}$ e os filtros MODWT são (157):

$$
\begin{aligned}
\tilde{W}_{j, t} & =\sum_{k=0}^{K_{j}-1} \tilde{h}_{j, k} X_{t-k \bmod \mathrm{N}} \\
\tilde{V}_{j, t} & =\sum_{k=0}^{K_{j}-1} \tilde{g}_{j, k} X_{t-k \bmod \mathrm{N}}
\end{aligned}
$$

em que $K_{j}=\left(2^{j}-1\right)(K-1)+1$.

Pelas formulações descritas, os coeficientes da wavelet MODWT em cada escala têm o mesmo comprimento que a série original $X$, sendo expressos em notação matricial como $\tilde{W}_{j}=\tilde{\omega}_{j} X$ e $\tilde{V}_{j}=\tilde{\nu}_{j} X$, onde cada linha da matriz $\left[\tilde{\omega}_{j}\right]_{N \times N}$ possui valores atribuídos por $\left\{\tilde{h}_{j, k}\right\}$. Enquanto que $\left[\tilde{\nu}_{j}\right]_{N \times N}$ possui valores atribuídos por $\left\{\tilde{g}_{j, k}\right\}$. Com isso tem-se (157):

$$
\tilde{\omega}_{j}=\frac{1}{2^{k}}\left[\begin{array}{cccccc}
\tilde{h}_{j, 0} & \tilde{h}_{j, N-1} & \tilde{h}_{j, N-2} & \cdots & \tilde{h}_{j, 2} & \tilde{h}_{j, 1} \\
\tilde{h}_{j, 1} & \tilde{h}_{j, 0} & \tilde{h}_{j, N-1} & \cdots & \tilde{h}_{j, 3} & \tilde{h}_{j, 2} \\
\vdots & \vdots & \vdots & \cdots & \vdots & \vdots \\
\tilde{h}_{j, N-2} & \tilde{h}_{j, N-3} & \tilde{h}_{j, N-4} & \cdots & \tilde{h}_{j, 0} & \tilde{h}_{j, N-1} \\
\tilde{h}_{j, N-1} & \tilde{h}_{j, N-2} & \tilde{h}_{j, N-3} & \cdots & \tilde{h}_{j, 1} & \tilde{h}_{j, 0}
\end{array}\right]
$$

Da mesma forma a matriz $\tilde{\nu}_{j}$ é expressa como acima, com cada $\left\{\tilde{h}_{j, k}\right\}$ substituído por $\left\{\tilde{g}_{j, k}\right\}$. Assim a série original $X$ pode ser expressa pela MODWT conforme:

$$
X=\sum_{j=1}^{J} \tilde{\omega}_{j}^{T} \tilde{W}_{j}+\tilde{\nu}_{J}^{T} \tilde{V}_{J}=\sum_{j=1}^{J} \tilde{d}_{j}+\tilde{S}_{J}
$$

que define a MODWT, baseada na Análise Multirresolução (MRA) de $X$, em termos dos j-ésimos componentes detalhes da MODWT, $\tilde{d}_{j}=\tilde{\omega}_{j}^{T} \tilde{W}_{j}$ e do J-ésimo componente de aproximação da MODWT $\tilde{S}_{J}=\tilde{\nu}_{J}^{T} \tilde{V}_{J}$.

A MODWT tem uma propriedade importante, que é ponto-chave no processamento de séries temporais não estacionárias. Sendo que $X_{t}$ é um processo estocástico, cuja equação pode ser escrita por meio do operador retroativo, $B^{s} X_{t}=X_{t-s}, s \geq 1$, de d-ésima order como $Y_{t}=(1-B)^{d} X_{t}=\sum_{k=0}^{d} \frac{d !}{k !(d-k) !}(-1)^{k} X_{t-k}$, determina-se que (157):

$$
\bar{W}_{j, t}=\sum_{k=0}^{K_{j}-1} \tilde{h}_{j, k} X_{t-k}, t \in Z
$$


em que $\left\{\bar{W}_{j, t}\right\}$ configura a saída obtida pela decomposição de $\left\{X_{t}\right\}$ empregando-se o filtro $\left\{\tilde{h}_{j, k}\right\}$ da wavelet MODWT.

\subsubsection{Wavelet Haar}

A wavelet Haar é um filtro de comprimento $L=2$. Ela representa a wavelet mais antiga e simples possível, sendo definida como:

$$
\Psi^{H}(t)= \begin{cases}1 & \text { para } 0 \leq t<1 / 2 \\ -1 & \text { para } 1 / 2 \leq t<1 \\ 0 & \text { caso contrário }\end{cases}
$$

cuja função escala é $\phi^{H}(t)=1,0 \leq t \leq 1 \mathrm{e}$

$$
\Psi_{j, k}^{H}(t)= \begin{cases}2^{-j / 2} & \text { para } 2^{j} k \leq t<2^{j}(k+1 / 2) \\ -2^{-j / 2} & \text { para } 2^{j}(k+1 / 2) \leq t<2^{j}(k+1) \\ 0 & \text { caso contrário }\end{cases}
$$

Pela equação 14 a wavelet de Haar é formulado como:

$$
\phi(t)=\phi(2 t)+\phi(2 t-1)=\frac{1}{\sqrt{2}} \sqrt{2} \phi(2 t)+\frac{1}{\sqrt{2}} \phi(2 t-1)
$$

Assim $h_{0}=-h_{1}=1 / \sqrt{2}$ e da mesma forma $g_{0}=-g_{1}=1 / \sqrt{2}$ (93).

A Figura 1 apresenta a wavelet Haar $\Psi^{H}(t)$.

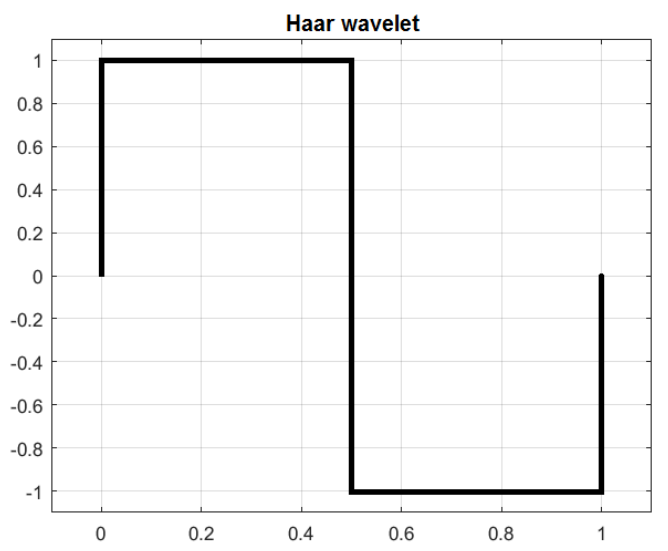

Figura 1 - Função wavelet Haar $\Psi^{H}(t)$. 


\subsection{MÉTODOS DE APRENDIZADO DE MÁQUINAS}

Diversos regressores foram propostos nos últimos anos, alguns utilizam árvores de regressão, outros redes neurais com regressão e existem algoritmos que se baseiam em modelos probabilísticos bayesianos.

\subsection{1 Árvores de Regressão}

As Árvores de Regressão são modelos preditivos não-lineares, que utilizam um treinamento supervisionado para a previsão de dados. Elas são idênticas as Árvores de Decisão porque elas também são representadas por um conjunto de nós de decisão (perguntas). Mas o resultado, ao contrário de uma categoria, é um escalar (87).

As Árvores de Regressão são compostas por dois tipos de nós: os nós internos da árvore, onde cada um desses nós corresponde a um teste feito em um dos atributos de entrada do conjunto de treinamento, e os nós-folha, onde são feitas as predições do atributo-meta. Os nós-folha de uma Árvore de Regressão possuem uma função matemática para predizer o atributo-meta (87).

\subsubsection{Regressão por Redes Neurais}

As Redes Neurais de Regressão são utilizadas para prever valores contínuos. Este modelo é frequentemente usado em áreas financeiras para Interpolação de curvas de juros.

Elas são formadas por um conjunto de nós de entrada, por uma ou mais camadas ocultas de neurônios e por uma camada de saída de neurônios. O sinal de entrada se propaga para a frente por meio da rede, de camada em camada. Cada camada possui sua própria matriz de pesos $w$, seu vetor polarizador $b$, um vetor de entrada $v$ e um vetor de saída $y$ (57).

Normalmente este modelo pode incluir também um viés aplicado externamente, representado por $b_{k}$. Esse viés tem o efeito de aumentar ou diminuir a entrada líquida da função de ativação, dependendo se ele é positivo ou negativo, respectivamente.

Em termos matemáticos, pode-se descrever um neurônio $k$ a partir das seguintes equações (57):

$$
u_{k}=\sum_{j=1}^{m} w_{k j} x_{j}
$$




$$
y_{k}=\varphi\left(u_{k}+b_{k}\right)
$$

sendo $x_{1}, x_{2}, \ldots, x_{m}$ os sinais de entrada; $w_{k 1}, w_{k 2}, \ldots, w_{k m}$ os pesos sinápticos do neurônio $k ; u_{k}$ a saída do combinador devido aos sinais de entrada; $b_{k}$ o viés; $\varphi($.) a função de ativação; e $y_{k}$ o sinal de saída do neurônio. O uso do viés $b_{k}$ tem o efeito de aplicar uma transformação afim à saída $u_{k}$ do combinador: $v_{k}=u_{k}+b_{k}$.

\subsubsection{Regressão por Aprendizagem Bayesiana}

Na Aprendizagem Bayesiana, Bayesian Learning (BL), a regra de Bayes de dependência é utilizada para calcular a distribuição de probabilidade a posteriori de $X_{i}$, dados os estados dos nós filhos de $Y$, representados por $X_{i}$, como (92):

$$
P\left(Y \mid X_{i}\right)=\frac{P\left(X_{i} \mid Y\right) \times P(Y)}{P\left(X_{i}\right)}
$$

\subsubsection{Regressão por k-Vizinhos mais Próximos}

Das técnicas da aprendizagem baseada em instâncias, a mais conhecida e referenciada na literatura é aquela que se baseia em critérios de vizinhança (10) (141) (9).

O algoritmo k-vizinhos mais próximos, k-Nearest Neighbors Algorithm (k-NN), é um método não-paramétrico utilizado para regressão. A entrada consiste em $k$ exemplos de treinamento mais próximos no espaço de características. Na regressão k-NN, a saída é o valor da propriedade para o objeto. Este valor representa a média dos valores dos seus $k$ vizinhos mais próximos.

O objetivo é gerar uma regressão pela interpolação dos pontos associados aos vizinhos mais próximos no conjunto de treinamento. Uma das possibilidades de algoritmo é aquela que usa a média ponderada pelo inverso da distância dos vizinhos mais próximos.

\subsection{MÁQUINAS DE SUPORTE VETORIAL}

Máquinas de Suporte Vetorial, Support Vector Machines (SVM), são máquinas lineares com implementação do método de Minimização do Risco Estrutural, que objetivam construir um hiperplano com superfície de decisão tal que a margem de separação entre exemplos positivos e negativos se tornem a máxima (144).

Um conceito importante da teoria de Vapnik e Chervonenkis (144) é a dimensão-VC, que representa uma medida da complexidade de um classificador estatístico, sendo definida como 
a maior cardinalidade de um conjunto de pontos que um classificador pode separar.

\subsubsection{SVM e as Margens Rígidas}

SVM lineares com margens rígidas definem fronteiras lineares a partir de dados linearmente separáveis. Seja $T$ um conjunto de treinamento com $n$ dados $x_{i} \in X$ e seus respectivos rótulos $y_{i} \in Y$, em que $X$ constitui o espaço dos dados e $Y=\{-1,+1\}$. $T$ é linearmente separável se é possível isolar os dados das classes +1 e -1 por um hiperplano (124) (17) de equação:

$$
f(x)=w \cdot x+b=0
$$

em que $w \cdot x$ é o produto escalar entre os vetores $w$ e $x, w \in X$ é o vetor normal ao hiperplano descrito e $\frac{b}{\|w\|}$ corresponde à distância do hiperplano em relação à origem, com $b \in \Re$.

Esta equação separa o espaço dos dados $X$ em duas regiões $w \cdot x+b>0$ e $w \cdot x+b<0$, conforme ilustra a Figura 2, obtida por meio do ambiente de programação para análise de dados estatísticos e gráficos $R$ (47).

A Figura 2 também destaca os vetores de suporte e as distâncias $\frac{b}{\|w\|}$ e $\frac{2}{\|w\|}$, sendo esta última a distância geométrica entre os dois hiperplanos.

Uma função sinal $g(x)=\operatorname{sgn}(f(x))$ pode então ser empregada na obtenção das classificações (132).

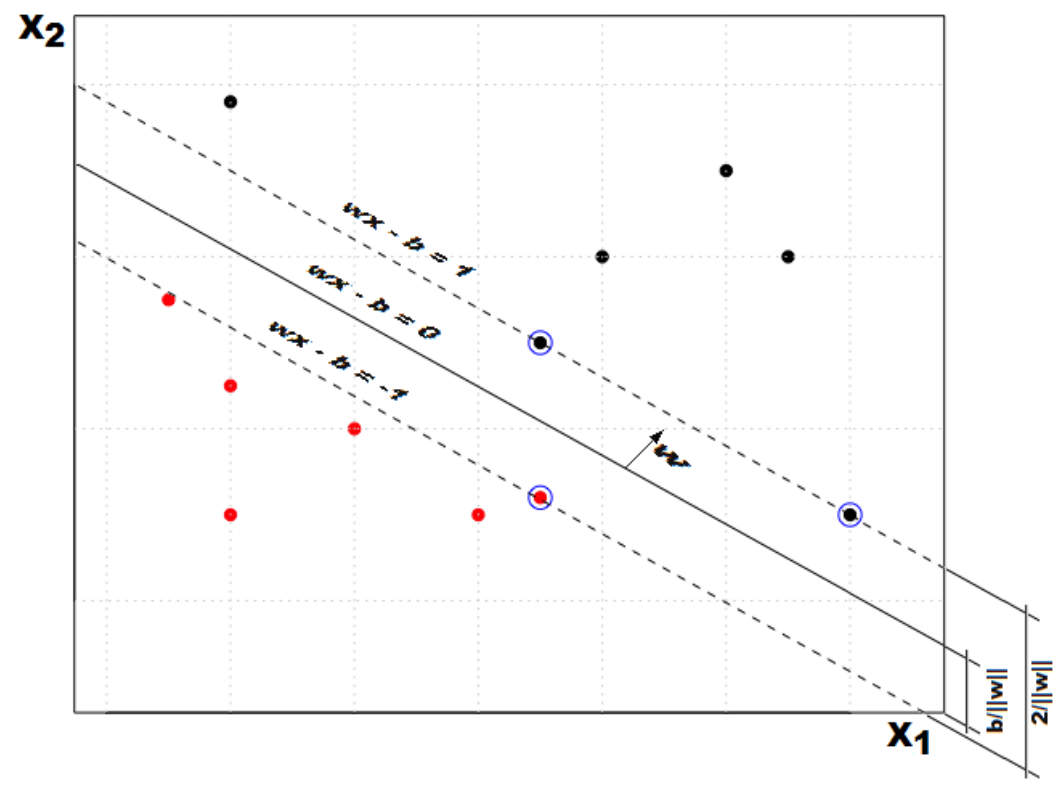

Figura 2 - Representação de um hiperplano de separação onde se destacam os vetores de suporte, as distâncias $\frac{b}{\|w\|}$ e $\frac{2}{\|w\|}$ e as regiões $w \cdot x+b>0$ e $w \cdot x+b<0$. 
A maximização da margem de separação dos dados em relação a $w \cdot x+b=0$ pode ser obtida pela minimização de $\|w\|$ (16).

Assim, pode-se definir o problema de otimização (124):

$$
\underset{w, b}{\operatorname{Minimizar}} \frac{1}{2}\|w\|^{2}
$$

seguindo a restrição $y_{i}=\left(w \cdot x_{i}+b\right)-1 \geq 0, \forall i=1, \ldots, n$, que pode ser solucionado por meio de uma função Lagrangiana (124) (110):

$$
\underset{\alpha}{\operatorname{Minimizar}} \sum_{i=1}^{n} \alpha_{i}-\frac{1}{2} \sum_{i, j=1}^{n} \alpha_{i} \alpha_{j} y_{i} y_{j}\left(x_{i} \cdot x_{j}\right)
$$

seguindo as restrições $\alpha_{i} \geq 0 \quad e \sum_{i=1}^{n} \alpha_{i} y_{i}=0$ para $\forall i=1, \ldots, n$.

Esta formulação denomina-se de forma dual, enquanto o problema original é referenciado como forma primal.

O classificador $g(x)$ é apresentado pela equação (124) (113):

$$
g(x)=\operatorname{sgn}(f(x))=\operatorname{sgn}\left(\sum_{x_{i} \in S V} y_{i} \alpha_{i}^{*} x_{i} \cdot x+b^{*}\right)
$$

em que sgn representa a função sinal, $w^{*}$ é fornecido pela equação:

$$
w^{*}=\sum_{i=1}^{n} \alpha_{i} y_{i} x_{i}
$$

e $b^{*}$ pela equação:

$$
b^{*}=\frac{1}{n_{S V}} \sum_{x_{j} \in S V}\left(\frac{1}{y_{j}}-\sum_{x_{i} \in S V} \alpha_{i}^{*} y_{i} x_{i} \cdot x_{j}\right)
$$

Esta função linear representa o hiperplano que separa os dados com maior margem e assim com melhor capacidade de generalização.

\subsubsection{SVM e as Margens Suaves}

Em situações reais é difícil encontrar aplicações cujos dados sejam linearmente separáveis. Por este motivo o SVM linear de margens rígidas é estendido para lidar com conjuntos de treinamento mais gerais, permitindo assim que alguns dados possam violar a restrição.

$$
y_{i}=\left(w \cdot x_{i}+b\right)-1 \geq 0, \forall i=1, \ldots, n
$$


Isso é possível pela a introdução de variáveis de folga $\xi_{i}$, para todo $i=1, \ldots, n$ (132), o que suaviza as margens do classificador linear (124):

$$
y_{i}=\left(w \cdot x_{i}+b\right) \geq 1-\xi_{i}, \quad \xi_{i} \geq 0, \forall i=1, \ldots, n
$$

A Figura 3, obtida por meio do ambiente de programação para análise de dados estatísticos e gráficos $R$ (47), exemplifica um hiperplano de separação onde se evidenciam os vetores de suporte e a variável de folga $\xi$, conforme a equação 47 .

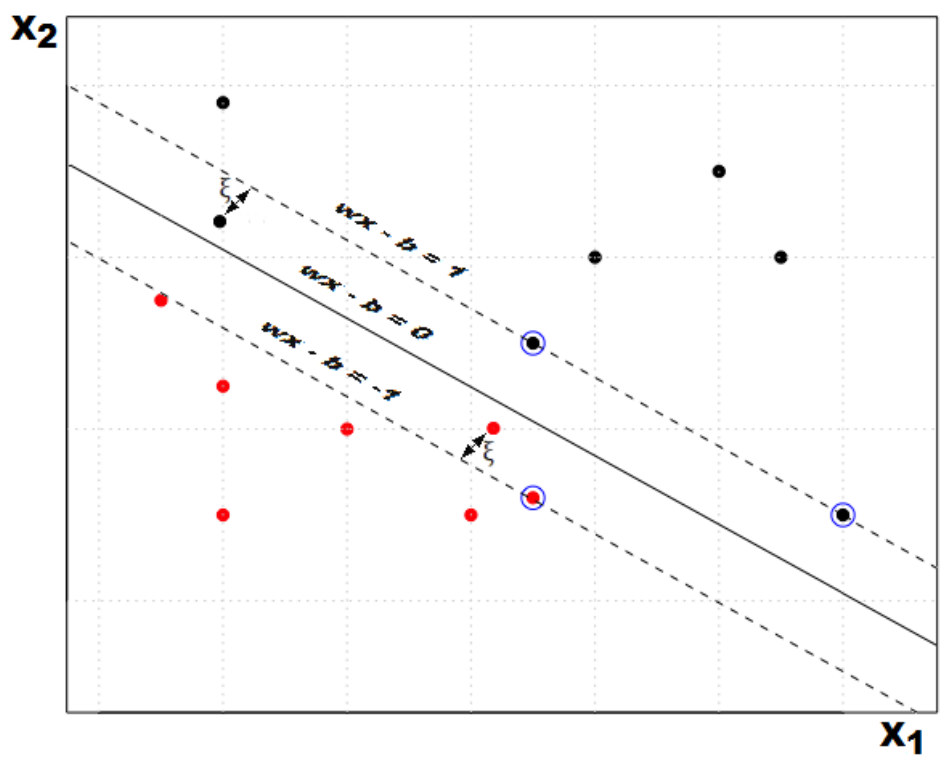

Figura 3 - Representação de um hiperplano de separação onde se evidenciam os vetores de suporte e a variável de folga $\xi$, conforme a equação 47.

Levando-se em consideração as variáveis de folga $\xi_{i}$, minimiza-se assim o erro sobre os dados de treinamento, e a função objetivo é definida como (16):

$$
\underset{w, b, \xi}{\operatorname{Minimizar}} \frac{1}{2}\|w\|^{2}+C\left(\sum_{i=1}^{n} \xi_{i}\right)
$$

onde a constante $C$ é um termo de regularização que impõe um peso à minimização dos erros no conjunto de treinamento em relação à minimização da complexidade do modelo (110).

Portanto, com a introdução de uma função Lagrangiana e tornando suas derivadas parciais nulas tem-se o problema dual:

$$
\underset{\alpha}{\operatorname{Minimizar}} \sum_{i=1}^{n} \alpha_{i}-\frac{1}{2} \sum_{i, j=1}^{n} \alpha_{i} \alpha_{j} y_{i} y_{j}\left(x_{i} \cdot x_{j}\right)
$$

seguindo as restrições $0 \leq \alpha_{i} \leq C$ e $\sum_{i=1}^{n} \alpha_{i} y_{i}=0$ para $\forall i=1, \ldots, n$. 
Com isso as variáveis $\xi_{i}^{*}$ podem ser calculadas conforme a equação:

$$
\xi_{i}^{*}=\max \left\{0,1-y_{i} \sum_{j=1}^{n} y_{j} \alpha_{j}^{*} x_{j} \cdot x_{i}+b^{*}\right\}
$$

sendo que a variável $b^{*}$ provém de $\alpha^{*}$ e de condições de Kühn-Tucker (113):

$$
\alpha_{i}^{*}\left[y_{i}\left(w_{i}^{*} \cdot x_{i}+b^{*}\right)-1+\xi_{i}^{*}\right]=0
$$

e

$$
\left(C-\alpha_{i}^{*}\right) \xi_{i}^{*}=0
$$

\subsubsection{SVM Não Lineares}

Em muitos casos não é possível separar acertadamente e satisfatoriamente os dados de treinamento por um hiperplano.

Mapeando-se um conjunto de treinamento de seu espaço original, referenciado como de entrada, para um novo espaço de dimensão maior, chamado de feature space ou espaço de características, o SVM tem a habilidade de lidar com problemas não lineares (58). Seja $\Phi$ : $X \rightarrow \Im$ um mapeamento em que $X$ é o espaço de entradas e $\Im$ é o espaço de características. A escolha apropriada de $\Phi$ faz com que o conjunto de treinamento mapeado em $\Im$ possa ser separado por um SVM linear.

Um exemplo é apresentado na Figura 4, em que o uso de uma fronteira curva seria mais adequada na separação das classes.

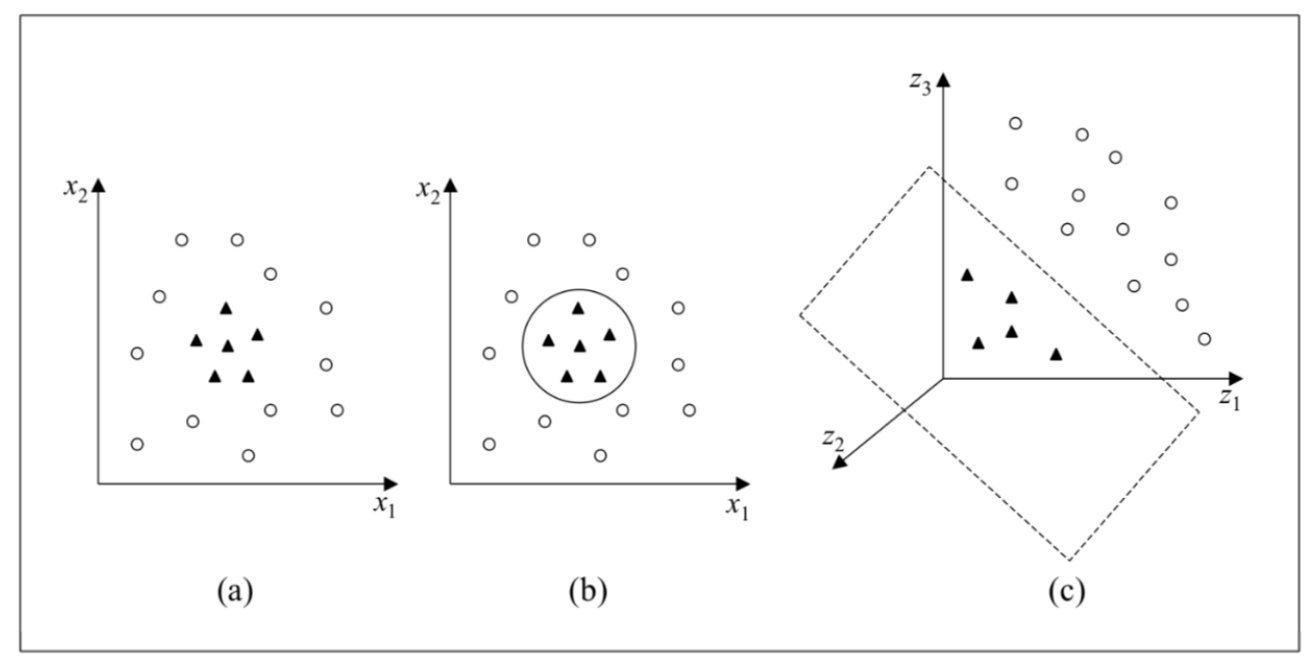

Figura 4 - Ilustração do conjunto de dados não lineares (a), da fronteira não linear no espaço de entradas (b) e da fronteira linear no espaço de características (c) (96). 
A motivação para o uso desse procedimento é dado pelo teorema de Cover (56). Considerandose um conjunto de dados não lineares, pela transformação dos dados de $\Re^{2}$ para $\Re^{3}$, tem-se o mapeamento representado pela equação:

$$
\Phi(x)=\Phi\left(x_{1}, x_{2}\right)=\left(x_{1}^{2}, \sqrt{2} x_{1} x_{2}, x_{2}^{2}\right)
$$

o que torna possível encontrar um hiperplano capaz de separar esses dados (96):

$$
f(x)=w \cdot \Phi(x)+b=0
$$

Para realizar o mapeamento aplica-se $\Phi$ aos exemplos presentes no problema de otimização, conforme a equação:

$$
\underset{\alpha}{\operatorname{Minimizar}} \sum_{i=1}^{n} \alpha_{i}-\frac{1}{2} \sum_{i, j=1}^{n} \alpha_{i} \alpha_{j} y_{i} y_{j}\left(\Phi\left(x_{i}\right) \cdot \Phi\left(x_{j}\right)\right)
$$

Assim o classificador extraído se torna:

$$
g(x)=\operatorname{sgn}(f(x))=\operatorname{sgn}\left(\sum_{x_{i} \in S V} \alpha_{i}^{*} y_{i} \Phi\left(x_{i}\right) \cdot \Phi(x)+b^{*}\right)
$$

onde $b^{*}$ pode ser calculado pela equação:

$$
b^{*}=\frac{1}{n_{S V: \alpha^{*}<C}} \sum_{x_{j} \in S V: \alpha_{j}^{*}<C}\left(\frac{1}{y_{j}}-\sum_{x_{i} \in S V} \alpha_{i}^{*} y_{i} \Phi\left(x_{i}\right) \cdot \Phi\left(x_{j}\right)\right)
$$

Pelas equações anteriores, a única informação necessária sobre o mapeamento é de como realizar o cálculo de produtos escalares entre os dados no espaço de características, pois tem-se $\Phi\left(x_{i}\right) \cdot \Phi\left(x_{j}\right)$, para dois dados $x_{i}, x_{j}$ em conjunto, produto que é obtido com o uso de funções Kernels (60):

$$
K\left(x_{i}, x_{j}\right)=\Phi\left(x_{i}\right) \cdot \Phi\left(x_{j}\right)
$$

Para o mapeamento dos dados de $\Re^{2}$ para $\Re^{3}$, apresentados no exemplo acima, o Kernel utilizado é (58):

$$
K\left(x_{i}, x_{j}\right)=\left(x_{1 i}^{2}, \sqrt{2} x_{1 i} x_{2 i}, x_{2 i}^{2}\right)\left(x_{1 j}^{2}, \sqrt{2} x_{1 j} x_{2 j}, x_{2 j}^{2}\right)=\left(x_{i} \cdot x_{j}\right)^{2}
$$

Para garantir a convexidade do problema de otimização e que o Kernel represente mapeamentos nos quais seja possível os cálculos de produtos escalares, utiliza-se funções Kernel que seguem as condições estabelecidas pelo teorema de Mercer (88) (68) (132) (60).

São exemplos de Kernels:

- Polinomial: $\left[\delta\left(x_{i} \cdot x_{j}\right)+\kappa\right]^{d}$ 
- Gaussiano ou Radial-Basis Function (RBF): $\exp \left(-\sigma\left\|x_{i} \cdot x_{j}\right\|^{2}\right)$ e

- Sigmoidal: $\tanh \left(\delta\left(x_{i} \cdot x_{j}\right)+\kappa\right)$.

\subsection{REGRESSÃO POR MEIO DE MÁQUINAS DE SUPORTE VETORIAL}

A definição matemática para Support Vector Regression (SVR), regressão por meio de Máquinas de Suporte Vetorial (SVM), para predição de séries temporais, pode ser expressa por (7) (38):

$$
\hat{y}_{t}=w_{t} \Phi\left(x_{t}\right)+b
$$

onde $\hat{y}_{t} \in \mathbb{R}$ é o valor predito da série temporal, $x_{t}=\left\{y_{t-D}, y_{t-D+1}, \ldots, y_{t-1}\right\}^{T} \in \mathbb{R}^{D}$ é o vetor de entrada do regressor e consiste nos dados históricos da série, $b \in \mathbb{R}$ é o termo de viés, $\omega_{t} \in \mathbb{R}^{M}$ é o vetor de pesos de $x_{t}$ e $\Phi\left(x_{t}\right) \mathbb{R}^{D} \rightarrow \mathbb{R}^{M}(M>D)$ é o espaço de características que transforma o vetor de entrada $x_{t} \in \mathbb{R}^{D}$ por meio do vetor de dimensão maior $\Phi\left(x_{t}\right) \in \mathbb{R}^{M}$.

A questão chave para resolver tal problema de previsão utilizando SVM é encontrar os valores ideais dos parâmetros SVM $\omega_{t}$ e $b$. Isto pode ser feito por meio da resolução de um problema de otimização.

De acordo com a função objetivo do problema de otimização, a solução pode ser obtida por meio do SVM empregando mínimos quadrados (LS-SVM). Assim, nas condições de Mercer, o regressor LS-SVM é determinado como (131):

$$
\hat{y}_{t}\left(x_{t}\right)=\sum_{i=1}^{N} \alpha_{i} K\left(x_{t}, x_{i}\right)+b
$$

onde $\alpha_{i}(i=1, \ldots, N)$ são os multiplicadores de Lagrange não negativos e $K\left(x_{t}, x_{i}\right)=$ $\Phi\left(x_{t}\right) \Phi\left(x_{i}\right)$ são as funções do Kernel. O presente trabalho se concentra na utilização do Kernel Gaussiano (RBF), conforme (131) (130):

$$
K\left(x_{t}, x_{i}\right)=\exp \left(-\sigma\left\|x_{t} \cdot x_{i}\right\|^{2}\right)
$$

\subsection{AJUSTES DE CURVAS}

Sendo uma coleção de observações feitas sequencialmente ao longo do tempo, as séries temporais são passíveis de modelagem matemática com propósitos determinados, tais como descrever o comportamento da série, investigar o mecanismo gerador da série temporal, fazer previsão de valores futuros e procurar periodicidades relevantes nos dados (14) (95). 
Estas modelagens são feitas no domínio do tempo e os mais frequentemente usados são os modelos de erro ou de regressão, descritos na seção Modelos Matemáticos e implementados computacionalmente por meio de Métodos de Aprendizado de Máquinas.

Para determinar qual modelo pode melhor representar uma série e posteriormente utilizá-la em previsões, pode-se seguir as etapas de: identificação do modelo, estimação, verificação e previsão (150).

$\mathrm{Na}$ abordagem de dados discretos o problema de ajuste de curvas dos pontos $\left(x_{1}, y_{1}\right)$, $\left(x_{2}, y_{2}\right), \ldots,\left(x_{n}, y_{n}\right)$, com $x_{i}$ pertencentes ao intervalo $[a, b]$, consiste em dadas $m+1$ funções $g_{0}(x), g_{1}(x), \ldots, g_{m}(x)$, contínuas em $[a, b]$, obter-se $m+1$ coeficientes $\beta_{0}, \beta_{1}$, $\ldots, \beta_{m}$ de tal forma que $f(x)=\beta_{0} g_{0}(x)+\beta_{1} g_{1}(x)+\ldots+\beta_{m} g_{m}(x)$ se aproxime de $y(x)$, fornecendo os valores $y_{1}, y_{2}, \ldots, y_{n}$ dos pontos da curva (6).

Uma idéia para que a função $f(x)$ se ajuste aos pontos $y_{i}$ é fazer com que o desvio, ou erro, $d_{i}=y_{i}-f\left(x_{i}\right)$ seja mínimo para todo $i=1,2, \ldots, n$. Assim, definindo uma medida mais abrangente que envolve a soma destes desvios elevados ao quadrado tem-se (134):

$$
D\left(\beta_{0}, \beta_{1}, \ldots, \beta_{m}\right)=\sum_{i=1}^{n}\left[y_{i}-f\left(x_{i}\right)\right]^{2}
$$

No ajuste linear simples a distribuição dos pontos no diagrama de dispersão assume aparência de uma reta, fazendo com que $g_{0}(x)=1, g_{1}(x)=x$ e $g_{2}(x)=g_{3}(x)=\ldots=g_{m}(x)=0$, cuja solução geral é (134):

$$
\beta_{1}=\frac{\left(n \cdot \sum x_{i} y_{i}-\sum x_{i} \cdot \sum y_{i}\right)}{\left(n \cdot \sum x_{i}^{2}-\left(\sum x_{i}\right)^{2}\right)}
$$

e

$$
\beta_{0}=\frac{\left(\sum y_{i}-\left(\sum x_{i}\right) \cdot \beta_{1}\right)}{n}
$$

No ajuste linear múltiplo a variável resposta depende de duas ou mais variáveis explicativas e o gráfico de dispersão apresenta um comportamento linear, fazendo com que $g_{0}(x)=1$, $g_{1}(x)=X_{1}, g_{2}(x)=X_{2}, \ldots, g_{m}(x)=X_{m}$.

No ajuste polinomial o diagrama de dispersão não apresenta as características lineares presentes nos outros tipos de ajuste. Nestas situações pode-se realizar o ajuste polinomial utilizando de funções $g_{i}(x): g_{0}(x)=1, g_{1}(x)=x, g_{2}(x)=x^{2}, \ldots, g_{m}(x)=x^{m}$.

\subsection{PREVISÕES}

A previsão de séries temporais é um método que utiliza um conjunto de valores históricos para prever um valor futuro. Este conjunto de valores históricos são observações igualmente 
espaçadas ao longo do tempo e podem representar dados como preço de ativos financeiros negociados nas bolsas de valores. Neste trabalho, estuda-se em especial os dados que compõem históricos de preços de moedas, um conjunto de ativos financeiros do tipo FOREX obtidos a partir de uma base de conhecimento público (116).

Na previsão baseada em dados históricos é feita uma análise de uma janela de tempo, contendo dados passados e presentes, para determinar pontos futuros. Tal janela é formada por um conjunto de pontos da série que devem trazer, juntamente com os dados pré-processados, informações suficientes para a determinação dos acontecimentos futuros. Dessa forma, a escolha do conjunto de pontos da série é de fundamental importância para sua melhor caracterização.

Dada uma série de tempo $\left\{X_{t}\right\}$ o objetivo é efetuar a previsão de $X_{T+h}, \hat{X}_{T}(h)$, de origem $T$ e horizonte $h$, ou seja, pretende-se prever $X_{T+h}$ tendo-se observações até o instante $T$. Dessa maneira, a previsão é dada pela esperança condicional de $X_{T+h}$ dado o passado $X_{T}, X_{T-1}, \ldots$.

Seja por exemplo um modelo ARIMA:

$$
\varphi(B)=\phi(B) \triangle^{d}=\left(1-\varphi_{1}(B)-\varphi_{2}(B)^{2}-\ldots-\varphi_{p+d}(B)^{p+d}\right) .
$$

A previsão através do Erro Quadrático Médio Mínimo (MMSE) de $X_{T+h}$ é definida como (94):

$$
\begin{aligned}
& \hat{X}_{T}(h)=\boldsymbol{E}\left[\varphi_{1} X_{T+h-1}+\cdots+\varphi_{p+d} X_{T+h-p-d}+\theta_{0}+\varepsilon_{T+h}-\theta_{1} \varepsilon_{T+h-1}-\cdots\right. \\
& \left.-\theta_{q} \varepsilon_{T+h-q} \mid X_{T}, X_{T-1}, \ldots\right] .
\end{aligned}
$$

Para se calcular as previsões segue-se os fatos (94):

1. $\boldsymbol{E}\left[X_{T+j} \mid X_{T}, X_{T-1}, \ldots\right]= \begin{cases}X_{T+j}, & \text { se } j \leq 0 \\ \hat{X}_{T}(j) & \text { se } j>0\end{cases}$

2. $\boldsymbol{E}\left[\varepsilon_{T+j} \mid X_{T}, X_{T-1}, \ldots\right]=\left\{\begin{array}{ll}\varepsilon_{T+j}, & \text { se } j \leq 0 \\ 0 & \text { se } j>0\end{array}\right.$.

Portanto, para se calcular previsões é necessário (94):

1. a substituição das esperanças passadas $(j \leq 0)$ por valores conhecidos, $X_{T+j}$ e $\varepsilon_{T+j}$; e

2. a substituição das esperanças futuras $(j>0)$ por previsões $\hat{X}_{T}(j)$ e 0 .

\subsection{ANÁLISE DOS MODELOS E MÉTODOS APRESENTADOS}

Algumas iniciativas de pesquisas acadêmicas vêm sendo desenvolvidas e realizadas sobre a utilização de métodos de aprendizado de máquinas e a teoria de aprendizado estatístico na 
regressão de séries temporais, das quais destacam-se conceitos relacionadas ao SVR, regressão por meio de Máquinas de Suporte Vetorial (SVM), à Transformada wavelet e aos Modelos Tradicionais.

Modelos tradicionais, como Box-Jenkins e modelos autorregressivos, assumem que as série temporais em estudo são geradas por meio de um processo linear (155). Tais modelos lineares podem ser compreendidos e analisados em maiores detalhes e são fáceis de explicar e implementar. Entretanto, seu uso é inapropriado em casos onde a série possui característica não-linear. Estudos afirmam que séries financeiras possuem ruídos e são não-lineares (11), inviabilizando o uso de tais modelos para este problema específico.

Nos últimos anos, um crescente número de estudos tem aparecido sobre o uso de métodos de aprendizado de máquinas em previsões de séries temporais financeiras. Algumas características explicam seu uso nesta área (155). Primeiro, são modelos auto-adaptativos, de modo que aprendem a partir de exemplos e capturam relações estruturais dos dados mesmo que essas sejam desconhecidas ou difíceis de descrever. Esses modelos são conhecidos por serem aproximadores universais de funções, sendo capazes de aproximar qualquer função contínua a qualquer precisão desejada. Outro fator importante é sua habilidade em reconhecer padrões não lineares nos dados das séries temporais, característica essa encontrada nos movimentos do mercado financeiro. Além disso, métodos de aprendizado de máquinas podem inferir dados não apresentados no processo de treinamento mesmo se os padrões apresentarem ruídos devido à sua grande capacidade de generalização.

No âmbito financeiro, exemplos de uso de redes neurais, aprendizagem bayesiana, algoritmo genético e máquinas de suporte vetorial não só se limitam ao problema da previsão, mas são utilizadas também no reconhecimento de padrões em gráficos financeiros, na estimação do preço de opções e na formação de indicadores de compra ou venda de um determinado ativo financeiro, sobretudo ações.

Modelos como Algoritmos Genéticos e Computação Evolutiva mostraram-se extremamente úteis na procura de padrões e na mineração de dados permitindo a realização de buscas globais, oferecendo uma melhor interação entre os atributos (42) (48) (43).

Utilizando-se dos Algoritmos Genéticos e das redes neurais do tipo Radial Basis Function (RBFNN) na predição de taxas de câmbio, Rivas (120) obteve um bom desempenho quando comparado com outros modelos. Nesta mesma linha de motivação as redes neurais e os algoritmos genéticos também mostraram-se com um melhor desempenho quando comparados às abordagens de modelagem de séries estatísticas (97).

Modelos como Standard Backpropagation (SBP), Scaled Conjugate Gradient (SCG) e Backpropagation with Baysian Regularization (BPR) são utilizados para prever taxas de câmbio (66). Outros modelos de redes neurais recorrentes mostram-se eficientes na otimização de 
rentabilidade de estratégias de negociação com base nos resultados de previsões (137).

Quando utilizados separadamente de modelos híbridos, as redes neurais se mostraram com dificuldade de aprendizagem para efetuar previsões de séries temporais não-estacionárias (49).

Os modelos de regressão baseados em SVM, Support Vector Regression (SVR), mostram-se adequados para resolver problemas de estimação não lineares de regressão. Estes modelos têm sido aplicados na solução de problemas de mineração de dados de séries temporais financeiras e têm-se mostrados eficientes na previsão deste tipo de séries temporais (143) (136) (19).

Outros trabalhos como em Lu (76) e Tsay (135) comprovaram que a utilização do SVM em um modelo híbrido para previsão de séries temporais, mostrou-se superior aos modelo de redes neurais artificiais para o tipo de problema envolvendo séries temporais financeiras que muitas vezes apresentam padrões de não linearidade.

Vários outros estudos utilizando-se de modelos híbridos foram apresentados, como por exemplo:

- A utilização de Self Organizing Map (SOM) que converte uma série temporal em uma sequência de símbolos como entrada de uma rede neural recorrente na geração de novas previsões (49). Entretanto o autor não comparou o desempenho do modelo proposto com outras abordagens de aprendizado de máquina, perdendo a oportunidade de verificar a eficácia de seu método.

- A utilização da abordagem de redes neurais com modelos ARIMA, aproveitando-se das caraterísticas individuais de cada modelo bem como dos resultados empíricos de seus trabalhos, Zhang (154) sugere que o modelo híbrido é melhor do que os dois modelos utilizados individualmente.

- O modelo híbrido ARIMA e SVM para a previsão do preço das ações (107).

- A metodologia híbrida que explora modelos Seasonal ARIMA (SARIMA) e SVM na previsão de séries temporais sazonais (21).

- O modelo híbrido formado por uma mistura de vários modelos de regressão de redes neurais, SVR e um conjunto selecionado de indicadores financeiros para efetuar a previsão de séries temporais de taxa de câmbio (102). O autor mostrou que a combinação de SVM e outros modelos eram superiores àquelas apresentadas nas previsões individuais.

Modelos para ajustes de séries temporais por meio de wavelets também foram propostos. Geralmente são trabalhos voltados para a análise matemática de modelos ajustados por meio de wavelets. 
Assim, apesar dos bons resultados encontrados nos diversos estudos recentes o desafio de encontrar modelos com melhor capacidade para previsão de dados ainda está em aberto, seja para previsão de ativos das bolsas de valores ou para previsão de tendências para indicadores financeiros.

Alguns modelos wavelets híbridos têm sido utilizados para prever os preços do petróleo (151), índice de ações (4), demanda de eletricidade (40) (152) (153) e outras séries de tempo. Mas, não há aplicações de SVM e wavelets para a previsão de taxa de câmbio.

Um modelo híbrido utilizando-se wavelets pode ser bem adequado para previsão de taxas de câmbio, pois as wavelets podem decompor as séries temporais em seus componentes de escala de tempo e esta pode ser uma estratégia muito bem sucedida na tentativa de desvendar a relação entre variáveis econômicas (118) e a previsibilidade da série.

Pela argumentação exposta neste capítulo, este trabalho propõe o desenvolvimento de um modelo híbrido de previsão de séries temporais que integra wavelets e SVR, regressão por meio de Máquinas de Suporte Vetorial (SVM), na previsão de séries financeiras, como uma alternativa aos modelos de previsão tradicionais. Oferece também uma comparação de desempenho deste novo modelo com os modelos ARFIMA, ARIMA, GARCH e o modelo SVR tradicional com Kernel, bem como sua aderência ao Expoente de Hurst por meio da estatística R/S. 


\section{DEFINIÇÃO DO MODELO SVR-WAVELET}

\subsection{MODELOS HÍBRIDOS}

Modelos híbridos, adotando técnicas de inteligência artificial, podem ser utilizados para otimizar o poder dos métodos de aprendizado de máquinas. Seu principal papel neste caso é encontrar as melhores configurações para um problema específico. Desde modo, eles automatizam o processo de tentativa e erro, que em geral costuma ser muito tedioso.

\subsubsection{O Modelo SVR-wavelet}

O modelo de previsão adaptativo está fundamentado na associação de modelos de wavelets e SVR, regressão por meio de Máquinas de Suporte Vetorial (SVM), ou seja, um sistema SVR-wavelet híbrido.

Este modelo utiliza da Transformada Discreta de wavelet (DWT) para efetuar a decomposição dos dados de séries de tempo, possibilitando com que componentes de alta e baixa frequências, contidos nos dados originais, possam ser separados.

Como resultado desta decomposição é possível identificar, por meio das baixas frequências, aspectos de tendências de longo prazo. Da mesma forma, por meio das altas frequências, é possível capturar descontinuidades, rupturas e singularidades contidas nos dados originais.

Após esta decomposição os componentes de aproximação e detalhes obtidos respectivamente das baixas e altas frequências, são utilizados como variáveis de entrada do SVR para prever dados futuros dos ativos financeiros.

A análise por meio de wavelets possibilita efetuar a eliminação de ruídos contidos nas séries por meio de filtros e técnicas de denoising ou wavelet shrinkage para obtenção de uma versão mais limpa da série temporal original. À isto é acrescentado o fato de que modelos não-lineares como as Máquinas de Suporte Vetorial, Support Vector Machines (SVM), são eficientes e eficazes na previsão de séries temporais.

Desta maneira o sistema híbrido SVR-wavelet pode ser considerado um otimizador adaptativo em que os coeficientes de alimentação são devidamente alterados para minimizar o Erro Quadrático Médio (MSE).

\subsubsection{Técnicas de Denoising ou wavelet Shrinkage}

As wavelets possibilitam a eliminação de ruídos por meio de técnicas de denoising ou wavelet shrinkage. A série temporal financeira pode ser alterada com a adição de ruído $r(n)$, resultando 
num sinal corrompido $y(n)$ :

$$
y(n)=x(n)+r(n)
$$

$\mathrm{Na}$ realidade, as séries temporais financeiras podem apresentar outros dois componentes: $T(n)$, tendência, e $S(n)$, sazonalidade. Este último componente não é encontrado nas séries FOREX estudadas por este trabalho.

Através da transformada wavelet, uma parte do ruído é removida da série temporal antes da extração de atributos (129). A técnica de wavelet denoising tenta transformar $y(n)$ novamente em $x(n)$, ou pelo menos num sinal similar. O processo é ilustrado na Figura 5.

sinal corrompido $y(n)$

sinal restaurado $x^{\prime}(n)$

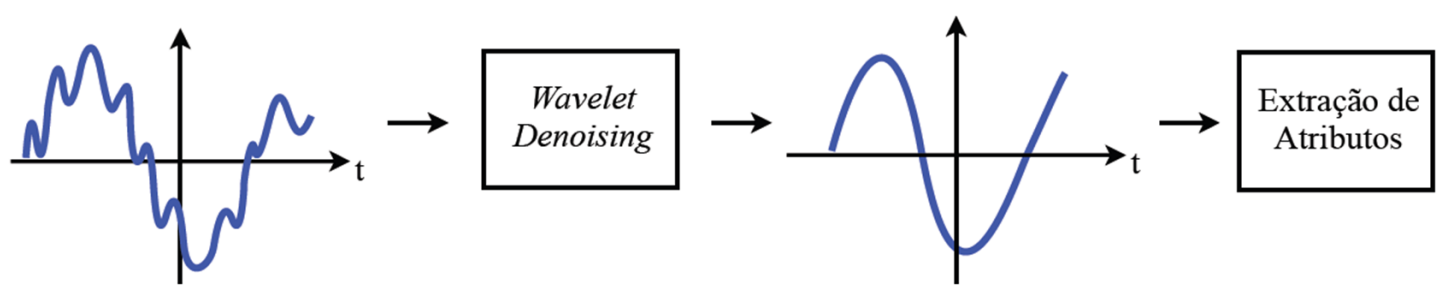

Figura 5 - Técnicas de denoising ou wavelet shrinkage (129).

Realizar a limpeza no domínio do tempo não é uma tarefa fácil tampouco prática, pois é complicado diferenciar o que é sinal do que é ruído em $y(n)$ (129). Uma alternativa inicial seria aplicar a transformada de Fourier discreta e fazer a análise no domínio da frequência.

A transformada wavelet é considerada como uma evolução da transformada de Fourier, trazendo flexibilidade para analisar séries temporais. A vantagem desta transformada é ser função de dois argumentos (129): um parâmetro (a) para alterar a escala horizontal da função mãe e um segundo parâmetro (b) para deslocar a função horizontalmente, trazendo uma informação de posição no tempo. A Figura 6 demonstra o efeito dessas modificações.
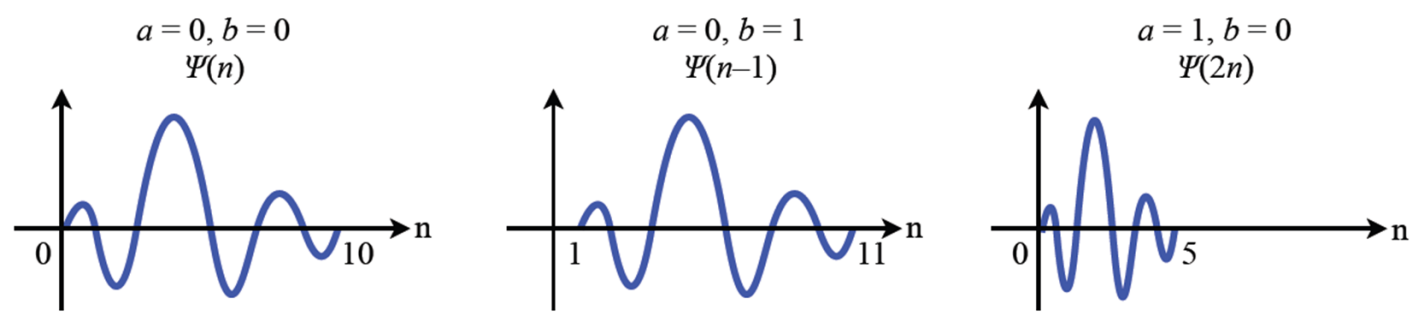

Figura 6 - Efeito das modificações proporcionadas pelos parâmetros (a) e (b) sob a função wavelet mãe (129). 
3.2 PREDIÇÃO DE SÉRIES TEMPORAIS POR MEIO DO MODELO SVR-WAVELET HÍBRIDO

Embora os modelos SVM empregando mínimos quadrados (LS-SVM) se mostrem com bom potencial na previsão de séries temporais, devido à sua capacidade de generalização, algumas séries podem apresentar um comportamento não-estacionário. Esta não-estacionariedade leva a pensar sobre a utilização de modelos híbridos como a combinação de SVM com a decomposição wavelet na predição de séries temporais. A solução pode ser obtida por meio da Transformada wavelet Discreta de Máxima Sobreposição (MODWT) como um pré-processador do conjuntos de dados de entrada.

Por intermédio de técnicas de denoising ou wavelet shrinkage efetua-se a eliminação de ruídos, possivelmente contidos nas séries financeiras. Em seguida, por meio da decomposição Multirresolução (MRA) a transformada wavelet é capaz de produzir novas subséries, cada uma fornecendo informações em uma escala de tempo específico. Em escalas de baixa resolução ou altas frequências, a transformada wavelet pode expressar características de curto prazo, enquanto em escalas de alta resolução ou baixas frequências, a função wavelet permite identificar o comportamento de longo prazo, tais como padrões de tendência e ciclos no conjunto de dados.

Conforme destacado neste trabalho, o princípio da análise wavelet é a de expressar ou aproximar um sinal ou função por uma família de funções geradas por dilatações e translações.

O presente trabalho salienta a utilização da wavelet Haar para a implementação da transformada wavelet. Entretanto, outras famílias de wavelet tais como as Daubechies, Coiflets e Gaussian podem ser utilizadas e analisadas para se verificar qual delas é mais adequada e aderente às séries temporais financeiras.

A Figura 7 ilustra as famílias de wavelet Haar, Daubechie de ordem 4 (db4), Coiflet de ordem 4 (coif4) e Gaussian de ordem 4 (gaus4) por meio do software MATLAB.

Seja $X$ um vetor de tamanho $N$ que contenha uma série de tempo $\left\{X_{t}\right\}$, e seja $J_{0}$ um número inteiro positivo. A MODWT mapeia o vetor de entrada de dados $X$ do domínio do tempo para a escala de tempo de wavelet gerando $J_{0}+1$ vetores $\tilde{W}_{j}, j=1, \ldots, J_{0}$ e $\tilde{V}_{J_{0}}$ cada uma de dimensão $N$. Os $\tilde{W}_{j}$ são composições de coeficientes da MODWT wavelet na escala $2^{j-1}$ enquanto que $\tilde{V}_{J_{0}}$ contém os coeficientes escalares da MODWT na escala $2^{J 0}$. Na forma matricial, os coeficientes da MODWT podem ser expressos por (4) (40) (151) (152):

$$
\left[\tilde{W}_{j}\right]_{N \times N}=\tilde{\omega}_{j} X, j=1, \ldots, J_{0}
$$



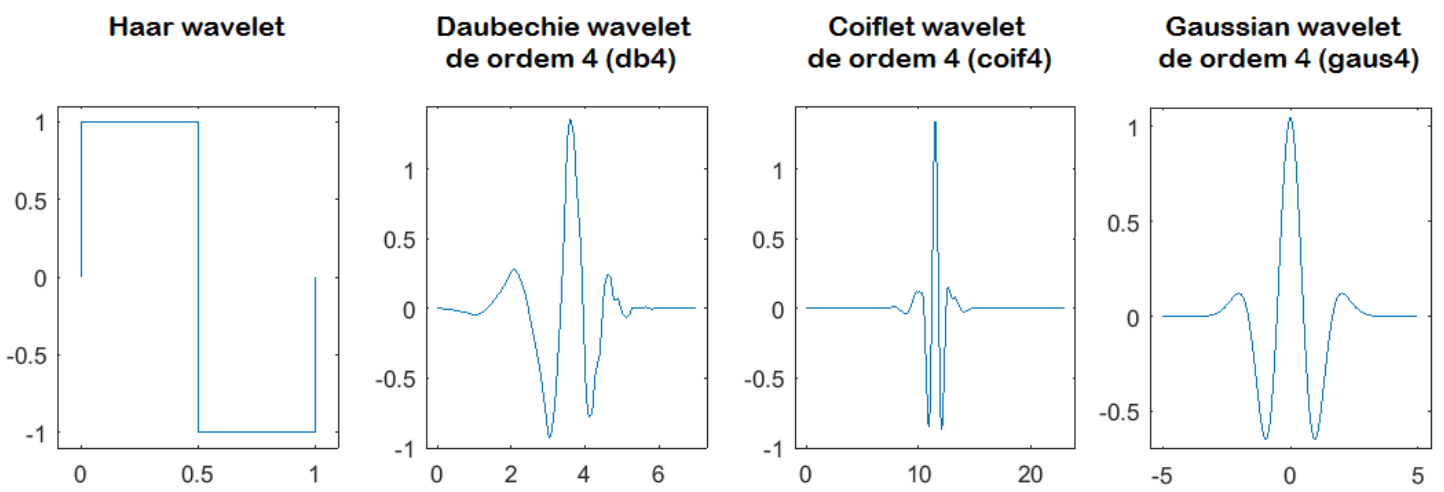

Figura 7 - Famílias de wavelet Haar, Daubechie de ordem 4 (db4), Coiflet de ordem 4 (coif4) e Gaussian de ordem 4 (gaus4) obtidas por meio do software MATLAB.

e

$$
\left[\tilde{V}_{J_{0}}\right]_{N \times N}=\tilde{\nu}_{J_{0}} X
$$

em que $\tilde{W}_{j}$ e $\tilde{V}_{J_{0}}$ são composições da wavelet MODWT e dos coeficientes de filtro escalar. Por intermédio da decomposição Multirresolução (MRA) o vetor original de entrada de $X$ pode ser calculado como:

$$
X=\sum_{j=1}^{J_{0}} \tilde{\omega}_{j}^{T} \tilde{W}_{j}+\tilde{\nu}_{J_{0}}^{T} \tilde{V}_{J_{0}}=\sum_{j=1}^{J_{0}} \tilde{d}_{j}+\tilde{S}_{J_{0}}
$$

em que os componentes $\tilde{d}_{j}, j=1, \ldots, J_{0}$, são as wavelet detalhes da subsérie gerada. $\mathrm{O}$ componente de aproximação $\tilde{S_{J_{0}}}=X-\sum_{j=1}^{J_{0}} \tilde{d}_{j}$ representa uma versão suavizada da série $\left\{X_{t}\right\}$ na escala $2^{J_{0}}$.

A Figura 8 ilustra a proposta do modelo para predição de séries temporais por meio do modelo SVR-wavelet híbrido.

Dada uma série de tempo $\left\{X_{t}\right\}$ o objetivo é efetuar a previsão de $X_{T+h}, \hat{X}_{T}(h)$, de origem $T$ e horizonte $h$.

O sistema de previsão proposto envolve quatro etapas: Primeiramente, efetua-se a eliminação de ruídos, possivelmente contidos nas séries financeiras do tipo FOREX, por meio de técnicas de denoising ou wavelet shrinkage. Depois, a MODWT expressa pela MRA é aplicada sobre o conjunto de dados de entrada e então é realizada a redução para o nível $J_{0}$. A série original é então representada como a soma dos componentes de aproximação $\tilde{S}_{J_{0}}$ e das wavelets detalhes $\tilde{d}_{j}, j=1, \ldots, J_{0}$.

Em seguida, ao invés de prever a série original, os componentes da MRA são previstos independentemente em cada escala de resolução por meio de um LS-SVM. 

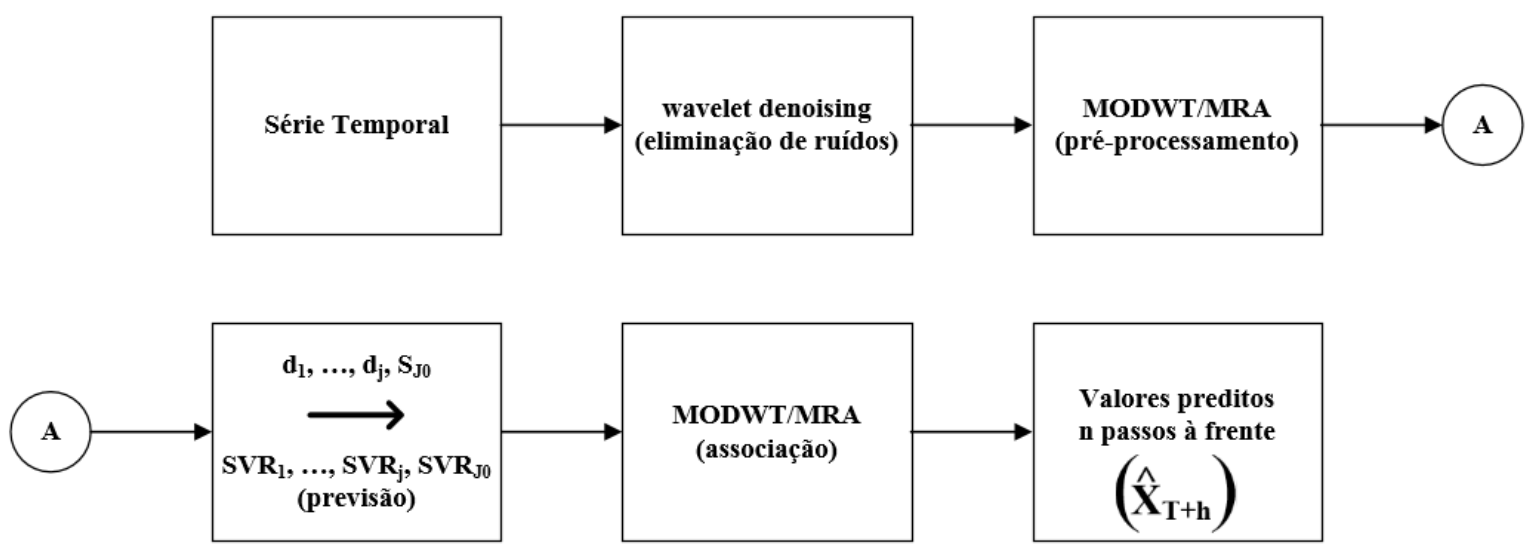

Figura 8 - Modelo proposto para predição de séries temporais por meio do modelo SVR-wavelet híbrido.

Por fim os componentes de aproximação $\tilde{S_{J_{0}}}$ e as wavelets detalhes $\tilde{d}_{j}, j=1, \ldots, J_{0}$, são recombinadas para gerar uma previsão agregada. Isto é feito utilizando-se da estrutura de adição da decomposição MRA, da seguinte maneira:

$$
\left\{\hat{X}_{t}\right\}=M R A_{\text {union }}\left\{\left[\hat{S}_{J_{0}}(t+1)\right]+\left[\sum_{j=1}^{J_{0}} \hat{d}_{j}(t+1)\right]\right\}
$$

onde $\hat{S}_{J_{0}}(t+1)=\left[\tilde{S}_{J_{0}}(t)+\tilde{S}_{J_{0}}(t-1)+\ldots+\tilde{S}_{J_{0}}(t-p)\right]$

e $\hat{d}_{j}(t+1)=\left[\tilde{d}_{j}(t)+\tilde{d}_{j}(t-1)+\ldots+\tilde{d}_{j}\left(t-q_{j}\right)\right], j=1, \ldots, J_{0}$.

em que as defasagens de tempo $p$ e $q_{j}, j=1, \ldots, J_{0}$, são aludidas como as dimensões dos componentes para estimativa dentro da estrutura de regressão LS-SVM.

A lógica por trás desta estratégia é que as séries temporais presentes no mundo real são geralmente não-estacionárias, com muito ruído e sujeitas à várias irregularidades. Além disso, elas podem conter fenômenos como tendências, ciclos, sazonalidades e flutuações de curto prazo. Explorando as propriedades inerentes à transformada wavelet, a MRA isola as dinâmicas de baixa frequência das dinâmicas de alta frequência, tendo como resultado um conjunto de subséries com mecanismos geradores mais fáceis. Por conseguinte, o treinamento do regressor LS-SVM para estas novas séries temporais é mais eficiente do que com os dados de entrada sem tratamento. 


\section{MÉTRICAS}

Com o objetivo de demonstrar que as séries temporais FOREX são não-viciadas e apropriadas para a comprovação do modelo híbrido SVR-wavelet, são propostos alguns testes estatísticos como os de normalidade e de raiz unitária para se comprovar que as séries em questão possuem distribuição não linear e também atestar o nível de correlação entre os períodos das séries.

Similarmente são apresentadas algumas métricas da precisão da previsão do modelo ajustado (142) (94) (127), das quais este trabalho destaca: Erro Quadrático Médio (MSE), Raiz Quadrada do Erro Quadrático Médio (RMSE), Erro absoluto médio (MAE), Erro Porcentual médio (MPE), Erro Porcentual Absoluto Médio (MAPE), Erro escalado Absoluto Médio (MASE), Autocorrelação de erros para uma defasagem (ACF1), Critério de Informação de Akaike (AIC), AIC de Segunda Ordem (AICc) e Critério de informação Bayesiano (BIC).

Suplementarmente aos testes, também se avalia a aderência ao Expoente de Hurst por meio da estatística R/S.

\subsection{TESTES DE NORMALIDADE}

Os testes de normalidade são utilizados para se constatar se a distribuição de probabilidade, associada às séries FOREX, pode ser aproximada pela distribuição normal, empregando-se os seguintes testes: Anderson-Darling, Cramer-von Mises, (Lilliefors) Kolmogorov-Smirnov, Pearson chi-square, Shapiro-Francia, Shapiro-Wilk e Jarque-Bera.

\subsubsection{Anderson-Darling}

O teste de Anderson-Darling é utilizado quando uma amostra de dados provém de uma população com uma distribuição específica, fazendo-se uso desta distribuição para calcular os valores críticos. Por um lado tem-se a vantagem de permitir um teste mais sensível, da outra a desvantagem de que os valores críticos devem ser calculados para cada distribuição. Sendo uma alternativa ao qui-quadrado e ao teste de Kolmogorov-Smirnov, o teste de Anderson-Darling pode ser definido como (64):

$$
A n=n \int_{-\infty}^{\infty} \frac{\left[F_{n}(x)-F_{0}(x)\right]^{2}}{F_{0}(x)\left(1-F_{0}(x)\right)} d F_{0}(x)
$$

em que $F_{n}$ é a distribuição empírica dos dados. Verifica-se que a distribuição de $A_{n}$ é dependente de $F_{0}$, o que contrasta com a estatística do teste de Kolmogorov-Smirnov. Considera-se apenas o caso uniforme em que $F_{0}(x)=x, 0 \leq x \leq 1$. 


\subsubsection{Cramer-von Mises}

Em geral, o teste Cramer-von Mises é empregado para testar a normalidade de qualquer variável e é um critério aplicado para apurar a qualidade do ajuste de uma função de distribuição cumulativa $F^{*}$ em comparação com uma dada função de distribuição empírica $F_{n}$, ou para comparar duas distribuições empíricas.

O teste de Cramer-von Mises determina se a hipótese nula é uma suposição razoável baseada na distribuição populacional de uma amostra aleatória $X$, para um dado nível de confiança. O teste é baseado em um processo de interpolação sob restrição com um nível de significância limitado a $0,001 \leq \alpha \leq 0,10$. A decisão de rejeitar a hipótese nula é tomada quando a estatística de teste excede o valor crítico.

Seja $S(x)$ a Função de Densidade Cumulativa Empírica (c.d.f.) estimada a partir do vetor de amostra $X$ e $F(x)$ a população normal correspondente à c.d.f., com média zero e desvio padrão unitário. A hipótese de Cramer-Von Mises e a estatística de teste são calculadas como (64):

$$
W^{2}=\int_{0}^{1}(S(x)-F(x))^{2} d F(x)
$$

onde $X$ é um vetor linha representando uma amostra aleatória. Em aplicações de uma amostra, $F^{*}$ é a distribuição teórica e $F_{n}$ é a distribuição empiricamente observada.

\subsection{3 (Lilliefors) Kolmogorov-Smirnov}

O teste de Kolmogorov-Smirnov, não paramétrico, pode ser usado para comparar uma amostra com uma distribuição de probabilidade de referência. A estatística KolmogorovSmirnov quantifica a distância entre a função de distribuição empírica da amostra da função de distribuição cumulativa da distribuição de referência.

No caso especial de testar a normalidade de uma distribuição, as amostras são padronizadas e comparadas com uma distribuição normal padrão, estabelecendo a média e a variância da distribuição de referência iguais às estimativas da amostra.

A função de distribuição empírica $F_{n}$ onde $n$ é independente e identicamente distribuído (i.i.d.) com observações $X_{i}$, é definida como (64):

$$
F_{n}(x)=1 / n \sum_{i=1}^{n} I_{[-\infty, x]}\left(X_{i}\right) \leq x
$$

em que $I_{[-\infty, x]}\left(X_{i}\right)$ é uma função indicadora, que é igual a 1 se $X_{i} \leq x$ e igual a 0 caso 
contrário. As estatísticas de Kolmogorov-Smirnov para um dado $F(x)$, c.d.f., é:

$$
D_{n}=\max _{x}\left|F_{n}(x)-F(x)\right|
$$

onde $\max _{x}$ representa a maior distância.

\subsubsection{Pearson chi-square}

Duas variáveis aleatórias $x$ e $y$ são independentes se a distribuição de probabilidade de uma das variáveis não for afetada pela presença de outra.

Suponha que $f_{i j}$ seja a frequência observada de eventos pertencentes tanto à $i$ - ésima categoria de $x$ quanto à $j$-ésima categoria de $y$. Suponha também que $e_{i j}$ seja a frequência esperada correspondente se $x$ e $y$ forem independentes. A hipótese nula da suposição de independência é rejeitada se o valor de $p$ da estatística de teste de Qui-quadrado, $\mathcal{X}^{2}$, definida como (64):

$$
\mathcal{X}^{2}=\sum_{i, j}^{n} \frac{\left(f_{i j}-e_{i j}\right)^{2}}{e_{i j}}
$$

for menor que um dado nível de significância.

\subsubsection{Shapiro-Wilk}

A estatística Shapiro-Wilk mede o afastamento da normalidade. Ela é calculada com valores de dados observados ordenados como (64):

$$
W^{2}=\frac{\left(\sum_{i=1}^{n} A_{i} X_{i}\right)^{2}}{\sum_{i=1}^{n}\left(X_{i}-\bar{X}\right)^{2}}
$$

em que $A_{i}=\left(a_{1}, \ldots, a_{n}\right)=\frac{m^{T} V^{-1}}{\left(m^{T} V^{-1} V^{-1} m^{T}\right)^{1 / 2}}$ são constantes, $m^{T}=\left(m_{1}, \ldots, m_{n}\right)^{T}$ são os valores esperados das estatísticas de ordem das variáveis aleatórias $i . i . d$ da distribuição normal padrão e $V$ é a matriz de covariância dessas estatísticas de ordem. A Hipótese é rejeitada se o valor $W_{2}$ for muito alto. 


\subsubsection{Shapiro-Francia}

O teste de Shapiro-Francia é uma simplificação do teste de Shapiro-Wilk para se comprovar a normalidade de uma população com base nos dados da amostra. Ele é definido como (64):

$$
W^{\prime}=\frac{\operatorname{cov}(x, m)}{\sigma_{x} \sigma_{m}}=\frac{\sum_{i=1}^{n}\left(x_{i}-\bar{x}\right)\left(m_{i}-\bar{m}\right)}{\sqrt{\left(\sum_{i=1}^{n}\left(x_{i}-\bar{x}\right)^{2}\right)\left(\sum_{i=1}^{n}\left(m_{i}-\bar{m}\right)^{2}\right)}}
$$

em que $x$ e $m$ são respectivamente a amostra tomada para o teste e $m$ sua média.

\subsubsection{Jarque-Bera}

A estatística de Jarque-Bera utiliza os terceiro e quarto momentos centrais como medidas da partida entre distribuição observada e distribuição normal. A estatística é calculada como (64):

$$
J B=\frac{N}{6}\left[S^{2}+1 / 4(K-3)^{2}\right]
$$

em que onde $N$ é o tamanho da amostra, $S$ é a assimetria da amostra, $K$ é a curtose da amostra.

\subsection{TESTES DE RAIZ UNITÁRIA}

Um dos propósitos de se analisar dados de séries temporais não-estacionárias é verificar a estacionariedade dos dados. Por este prisma adota-se os conseguintes testes, amplamente utilizados em estatística.

\subsubsection{Dickey-Fuller Aumentado}

O teste Dickey-Fuller Aumentado é um teste para uma raiz unitária em uma amostra de séries temporais. Sendo uma versão aumentada do teste Dickey-Fuller para um conjunto maior de modelos de séries temporais, este teste apresenta-se com um número estatístico negativo e quanto mais negativo for o número mais forte é a tendência de se rejeitar a hipótese de que existe uma raiz unitária a um dado nível de confiança. O procedimento para este teste é o mesmo que para o teste Dickey-Fuller, conforme (114):

$$
\Delta y_{t}=\alpha+\beta_{t}+\gamma y_{t-1}+\delta_{1} \Delta y_{t-1}+\cdots+\Delta y_{t-p+1}+\varepsilon_{t}
$$


Onde $\alpha$ é uma constante, $\beta$ é o coeficiente de uma tendência temporal e $p$ é a ordem da defasagem do processo autoregressivo. Lembrando que estabelecendo as restrições $\alpha=0$ e $\beta=0$, corresponder-se-à modelagem de um Passeio Aleatório, e que empregando-se a restrição $\beta=0$, corresponder-se-à modelagem de um Passeio Aleatório com deslocamento.

Ao se integrar as defasagens de ordem $p$, a formulação Dickey-Fuller Aumentado possibilita processos de autorregressão de ordem superior, o que exprime que o tamanho da defasagem $p$ deva ser determinado, quando se aplica o teste por meio da estatística AIC.

\subsubsection{Phillips-Perron}

Outro teste de raiz unitária largamente utilizado em análise de séries temporais. Ele baseiase no teste Dickey-Fuller da hipótese nula $\delta=0$ em $\Delta y_{t}=\delta y_{t-1}+u_{t}$, primeiro operador diferença. Tal como no teste Dickey-Fuller Aumentado, o teste Phillips-Perron aborda a questão de que o processo de geração de dados para $y_{t-1}$ pode ter uma ordem mais elevada de autocorrelação. O teste Phillips-Perron aborda esta questão, introduzindo defasagens de $\Delta y_{t}$ como regressores na equação de teste (78).

$$
\Delta y_{t}=\beta^{\prime} \mathcal{D}_{t}+\delta y_{t-1}+u_{t}
$$

onde $u_{t}$ tem ordem de integração $0, I(0)$.

\subsubsection{KPSS}

Possivelmente o teste mais conhecido para a estacionariedade em Econometria, o teste KPSS, introduzido por Kwiatkowski, Phillips, Schmidt e Shin em 1992, rejeita a hipótese de estacionariedade como verdadeira, o que conduz à preferência pela hipótese de não estacionariedade da raiz unitária (53).

$$
K P S S=1 / T^{2} \frac{\sum_{t=1}^{T} S_{t}^{2}}{\hat{\sigma}_{\infty}^{2}}
$$

em que $S_{t}=\sum_{s=1}^{T} \hat{e}_{s}$, onde $\hat{e}_{t}$ é o resíduo da regressão e $\hat{\sigma}_{\infty}^{2}$ é um estimador consistente de autocorrelação com variância $\hat{e}_{t}$. 


\subsection{TESTES DE NÃO-LINEARIDADE}

\subsubsection{Terasvirta Neural Network}

Esse tipo de teste de linearidade para séries temporais foi introduzido com base em conceitos da teoria das redes neurais (138), podendo ser equacionado como (33):

$$
u_{t}=\phi_{1} \nabla\left(\alpha_{0}+A^{T} X_{t-1}\right)-1 / 3 \sum_{j_{1}=1}^{n} \sum_{j_{2}=j_{1}}^{n} \sum_{j_{2}=j_{3}}^{n} \phi_{2_{j_{1} j_{2} j_{3} x_{j_{1}} x_{j_{2}} x_{j_{3}}}}+u_{t}^{*}
$$

\subsubsection{White Neural Network}

Um outro teste de linearidade foi introduzido por White em 1989 (147). White desenvolveu um teste para não-linearidade negligenciada, fundamentando-se no teste de rede neural em que uma função de teste $h\left(x_{t}\right)$ selecionada entre as ativações das camadas internas $\psi\left(x_{t} \Gamma_{j}\right)$, $j=1, \ldots, q$, onde $\Gamma_{j}$ são vetores colunas, aleatórios e independentes de $x_{t}$, sendo expresso como (70):

$$
\boldsymbol{E}\left[\psi\left(x_{t} \Gamma_{j}\right) \varepsilon_{t}^{*} \mid \Gamma_{j}\right]=\boldsymbol{E}\left[\psi\left(x_{t} \Gamma_{j}\right) \varepsilon_{t}^{*}\right]=0, j=1, \ldots, q
$$

em que $\boldsymbol{E}\left(\Psi_{t} \varepsilon_{t}^{*}\right)$ para $H_{0}$ e $\Psi_{t}=\left(\psi\left(x_{t} \Gamma_{1}\right), \ldots, \psi\left(x_{t} \Gamma_{q}\right)\right)^{\prime}$ é o vetor de ativação das camadas internas.

Em condições de regularidade adequadas, conserva-se o teorema do limite central e se dispondo de um estimador consistente para a matriz de covariância assintótica, $\hat{W}_{n}$, a estatística assintótica de qui-quadrado pode ser equacionada como (70):

$$
\left(n^{-1 / 2} \sum_{t=1}^{n} \psi \hat{\varepsilon}_{t}\right)^{\prime} \hat{W}_{n}^{-1}\left(n^{-1 / 2} \sum_{t=1}^{n} \psi \hat{\varepsilon}_{t}\right) \underset{\rightarrow}{\rightarrow} \mathcal{X}^{2}\left(q^{*}\right)
$$

Realizando-se um teste em $q^{*}<q$ nos componentes principais de $\Psi_{t}$, não colineares com $x_{t}$, por exemplo $\Psi_{t}^{*}$, e empregando-e a estatística de teste equivalente, $N_{q, q^{*}}$, tem-se que (70):

$$
N_{q, q^{*}} \equiv n R^{2} \underset{\rightarrow}{\rightarrow} \mathcal{X}^{2}\left(q^{*}\right)
$$

onde $R^{2}$ é uma correlação múltipla quadrada não centrada da regressão linear padrão de $\hat{\varepsilon}_{t}$ em $\Psi_{t}^{*}$ e $x_{t}$. 


\subsection{TESTES DE CORRELAÇÃO PELO GRÁFICO DE DISPERSÃO}

O gráfico de dispersão mostra as relações ou associações entre duas variáveis quantitativas. A relação entre duas variáveis é chamada de correlação (20).

Segundo Michael (44) o gráfico de dispersão é usado para verificar se existe relação de causa e efeito entre duas variáveis de natureza quantitativa determinando se existe relação e qual a intensidade da relação entre elas.

O gráfico de dispersão possibilita construir uma regressão linear, determinando-se uma reta que aponta a relação entre duas variáveis e indica a função que dá o comportamento da relação entre elas.

Quando o valor de uma variável cai com o aumento do valor de outra variável, diz-se que as variáveis são negativamente correlacionadas. E quando o valor de uma variável sobe com o aumento do valor de outra variável, diz-se que as variáveis são positivamente correlacionadas.

\subsection{MSE, RMSE, MAE, MPE, MAPE, MASE, ACF1, AIC, AICC, BIC}

\subsubsection{MSE - Erro Quadrático Médio}

O Erro Quadrático Médio ou Mean Squared Error (MSE), é a soma das diferenças entre o valor estimado e o valor real dos dados, ponderados pelo número de termos. Com tal característica, o ME dos valores previstos $\hat{y}_{t}$ das observações no instante $t$ da variável dependente da regressão $y_{t}$ é avaliado para $n$ diferentes previsões como (123):

$$
M S E=\frac{\sum_{t=1}^{n}\left(y_{t}-\hat{y}_{t}\right)^{2}}{n}
$$

\subsubsection{RMSE - Raiz Quadrada do Erro Quadrático Médio}

A Raiz Quadrada do Erro Quadrático Médio ou Root Mean Square Error (RMSE) é uma medida das diferenças entre valores preditos por um modelo e os valores realmente observados. Ele equivale ao desvio padrão da amostra das diferenças entre valores previstos e valores observados. Essas diferenças individuais são chamadas de resíduos.

Deste modo RMSE dos valores previstos $\hat{y}_{t}$ das observações no instante $t$ da variável dependente da regressão $y_{t}$ é calculado para $n$ diferentes previsões como a raiz quadrada da média 
dos quadrados dos desvios (115):

$$
R M S E=\sqrt{\frac{\sum_{t=1}^{n}\left(y_{t}-\hat{y}_{t}\right)^{2}}{n}}
$$

\subsubsection{MAE - Erro Absoluto Médio}

O Erro Absoluto Médio ou Mean Absolute Error (MAE), é a média de todos os erros absolutos, diferença entre valores ajustados $\hat{y}_{t}$ das observações no instante $t$ da variável dependente da regressão $y_{t}$, sendo determinado como (122):

$$
M A E=\frac{\sum_{t=1}^{n}\left|y_{t}-\hat{y}_{t}\right|}{n}
$$

onde $n$ é o tamanho da série.

\subsubsection{MPE - Erro Porcentual Médio}

O Erro Porcentual Médio ou Mean Percentage Error (MPE), é a média calculada de erros percentuais pelos quais as previsões do modelo diferem das observações no instante $t$ da variável dependente da regressão $y_{t}$. É computado como (139):

$$
M P E=\frac{100 \%}{n} \sum_{t=1}^{n} \frac{y_{t}-\hat{y}_{t}}{y_{t}}
$$

onde $y_{t}$ são as observações no instante $t$ da variável dependente da regressão, $\hat{y}_{t}$ são os valores ajustados e $n$ é o tamanho da série.

\subsubsection{MAPE - Erro Porcentual Absoluto Médio}

O Erro Porcentual Absoluto Médio ou Mean Absolute Percentage Error (MAPE), é uma medida da precisão de previsão na estimativa de tendência e pode ser formulado como (139):

$$
M A P E=\frac{100 \%}{n} \sum_{t=1}^{n}\left|\frac{y_{t}-\hat{y}_{t}}{y_{t}}\right|
$$

onde $y_{t}$ são as observações no instante $t$ da variável dependente da regressão, $\hat{y}_{t}$ são os valores ajustados e $n$ é o tamanho da série. 


\subsubsection{MASE - Erro Escalado Absoluto Médio}

O Erro Escalado Absoluto Médio ou Mean Absolute Scaled Error (MASE), é uma medida da precisão das previsões e pode ser avaliado como (105):

$$
M A S E=\frac{1}{n} \sum_{t=1}^{n}\left(\frac{\left|e_{t}\right|}{\frac{1}{n-1} \sum_{t=2}^{n}\left|y_{t}-y_{t-1}\right|}\right)
$$

em que o numerador $e_{t}$ é o erro de previsão para um determinado período, no qual $y_{t}$ são as observações no instante $t$ da variável dependente da regressão, $\hat{y}_{t}$ são os valores ajustados, e $n$ é o tamanho da série. O denominador representa o Erro Absoluto Médio (MAE) da previsão para um período à frente.

\subsubsection{ACF1 - Autocorrelação de Erros para uma Defasagem}

A Autocorrelação de Erros para uma Defasagem ou Autocorrelation of Errors at Lag 1 (ACF1), mede a autocorrelação dos resíduos para uma defasagem. Para duas variáveis $y_{1}$ e $y_{2}$, o ACF1 é calculado como (94):

$$
\rho=\frac{\boldsymbol{E}\left(y_{1}-\mu_{1}\right)\left(y_{2}-\mu_{2}\right)}{\sigma_{1} \sigma_{2}}=\frac{\operatorname{cov}\left(y_{1}-y_{2}\right)}{\sigma_{1} \sigma_{2}}
$$

em que $\boldsymbol{E}$ é o operador esperança, $\mu_{1}$ e $\mu_{2}$ são respectivamente as médias para as variáveis $y_{1}$ e $y_{2}$, e $\sigma_{1}$ e $\sigma_{2}$ são seus desvios padrão.

\subsubsection{AIC - Critério de Informação de Akaike}

O Critério de Informação de Akaike ou Akaike Information Criterion (AIC), é uma medida relativa da quantidade de ajustes de um modelo estatístico estimado. Dada uma coleção de modelos para os dados, AIC estima a qualidade de cada modelo, em relação a cada um dos outros modelos. Portanto, o AIC fornece um meio para a seleção do modelo, sendo determinado como (156) (35):

$$
A I C=2 k-2 \ln (\hat{L})
$$

em que $k$ é o número de parâmetros estimados no modelo e $\hat{L}$ é o valor maximizado da função de verossimilhança do modelo estudado. 


\subsubsection{AICc - AIC de Segunda Ordem}

O AIC de Segunda Ordem ou Second-Order AIC (AICc) é o AIC com uma correção para tamanhos de amostra finitos. A fórmula para AICc depende do modelo estatístico. Supondo que o modelo é univariante, linear e tem resíduos distribuídos normalmente, o AICc é computado como (156):

$$
A I C c=A I C+\frac{2 k(k+1)}{n-k-1}
$$

em que $A I C$ é o Critério de Informação de Akaike, $k$ é o número de parâmetros estimados no modelo, $\hat{L}$ é o valor maximizado da função de verossimilhança do modelo estudado e $n$ indica o tamanho da amostra.

\subsubsection{BIC - Critério de Informação Bayesiano}

Critério de Informação Bayesiano ou Bayesian Information Criterion (BIC), é um critério para a avaliação de modelos definido em termos de probabilidade a posteriori. É formulado como (156) (35):

$$
B I C=\ln (n) k-2 \ln (\hat{L})
$$

em que $\hat{L}$ é o valor maximizado da função de verossimilhança do modelo estudado, $n$ é o número de observações ou o tamanho da amostra e $k$ é o numero de parâmetros estimados.

\subsection{INTERPRETAÇÃO DOS P-VALUES}

Em estatística, o p-value, chamado de nível descritivo ou probabilidade de significância, é a probabilidade de se obter uma estatística de teste igual ou mais extrema que aquela observada em uma amostra, sob a hipótese nula. O p-value está diretamente relacionado ao tamanho da amostra. A Tabela 1 apresenta uma interpretação admissível dos p-values (3). Os valores estatísticos de p-values mostrados na Tabela 1 são considerados razoáveis pois a quantidade de pontos amostrados neste trabalho são superiores a 3000 pontos por série estudada.

Usualmente define-se duas hipóteses, a nula, $H_{0}$, e a alternativa, $H_{A}$. Em estatística convenciona-se definir a hipótese alternativa como a hipótese proposta pelo pesquisador, ao passo que a hipótese nula é o seu complemento. Inicialmente, a hipótese nula é considerada a verdadeira. Ao se confrontar a hipótese nula com os obtidos de uma amostra aleatória tomada de uma população de interesse, verifica-se sua legitimidade em termos probabilísticos, o que 
Tabela 1 - Interpretação admissível dos p-values (3).

\begin{tabular}{|l|l|}
\hline p-value & Interpretação \\
\hline \hline$p<0,01$ & evidência muito forte contra $H_{0}$ \\
\hline $0,01 \leq p<0,05$ & evidência moderada contra $H_{0}$ \\
\hline $0,05 \leq p<0,10$ & evidência sugestiva contra $H_{0}$ \\
\hline $0,10<p$ & pouca ou nenhuma evidência real contra $H_{0}$ \\
\hline
\end{tabular}

leva a rejeitar-se ou não $H_{0}$. Se não se rejeita $H_{0}$, então ela é considerada como verdadeira; caso contrário, adota-se $H_{A}$ como verdadeira.

\subsection{CRITÉRIOS PARA SELEÇÃO DO MELHOR MODELO}

A escolha do modelo apropriado, sob a luz da estatística, é uma questão excessivamente indispensável na análise de dados (15). Buscam-se modelos mais parcimoniosos ou aqueles que envolvam o mínimo de parâmetros possíveis a serem estimados e que expliquem bem o comportamento da variável resposta.

Nesta linha, diversos critérios para seleção de modelos são apresentados na literatura (15) (148) (75). Dentre os critérios para seleção de modelos, os critérios baseados na Estimativa por Máxima Verossimilhança (MLE) são os mais empregados, com maior ênfase ao Critério de Informação de Akaike (AIC), ao Critério AIC de Segunda Ordem (AICc) e ao Critério de Informação Bayesiano (BIC).

Os três critérios, apesar de conceitualmente diferentes acerca dos modelos em avaliação, utilizam o mesmo critério estatístico, o máximo da função de verossimilhança como medida do ajuste, contudo, definem valores críticos diferentes. Esta é a diferença fundamental entre os três métodos.

Com o teste da razão de verossimilhança, considera-se por hipótese que o modelo mais simples é o de melhor ajuste, até que se observem, dado um nível de significância, diferenças estatísticas para um modelo mais completo.

\subsection{EXPOENTE DE HUST E A ANÁLISE R/S}

É possível encontrar características fractais em séries temporais de taxas de câmbio por meio da análise dos intervalos de tempo de amostras diárias e intra-diárias (2) (25).

A estatística de Reescalonamento R/S, Rescaled Range, de Hurst é uma métrica que permite verificar a existência da dinâmica fractal numa série e investigar a presença de memória longa 
persistente, anti-persistente ou a identificação de passeio aleatório (94) (2).

O Expoente de Hust foi sugerido pelo engenheiro e hidrólogo inglês Harold Hurst em 1951, que inspirando-se nas definições de processo aleatório sugeridas por Einstein, obteve o Expoente de Hurst $(H)$, parte da estatística R/S (62) (28). Estudado por Hurst, se a série de valores do nível do rio Nilo se configurasse como um passeio aleatório, o valor do desvio padrão seria igual a $\sigma_{\varepsilon} t^{\frac{1}{2}}$.

O Expoente de Hurst $(H)$ fornece informações concretas sobre correlação e persistência, o que torna $H$ um excelente índice para estudar processos complexos nomeadamente as séries temporais financeiras, estudadas neste trabalho. Os valores do Expoente de Hurst das séries temporais são estimados por intermédio do método Análise R/S (117) (39).

$\mathrm{O}$ valor deste expoente varia entre 0 e 1 . Quanto mais distintos de 0.5 , maior é a memória de longo prazo. Portanto, são processos de memória longa aqueles processos que tenham $H>0.5$, processos persistentes, ou $H<0.5$, processos anti-persistentes. Para $H=0.5$ o sinal ou processo é aleatório ou ainda um Movimento Browniano. Consequentemente, a Estatística R/S, introduzida por Hurst (1951), tem como propósito testar a existência de memória longa em séries temporais.

Seja uma sequência de valores $\varepsilon_{t}, t \geq 1$, independentes e identicamente distribuídos (i.i.d.), com média $\mu_{\varepsilon}$ e variância $\sigma_{\varepsilon}^{2}$. A sequência $W_{t}=\varepsilon_{1}+\varepsilon_{2}+\cdots+\varepsilon_{t}$ é definida como um passeio aleatório e também:

$$
\begin{gathered}
\boldsymbol{E}\left(W_{t}\right)=\boldsymbol{E}\left(\varepsilon_{1}\right)+\boldsymbol{E}\left(\varepsilon_{2}\right)+\cdots+\boldsymbol{E}\left(\varepsilon_{t}\right)=t \mu_{\varepsilon} \\
\operatorname{Var}\left(W_{t}\right)=\operatorname{Var}\left(\varepsilon_{1}\right)+\operatorname{Var}\left(\varepsilon_{2}\right)+\cdots+\operatorname{Var}\left(\varepsilon_{t}\right)=t \sigma_{\varepsilon}^{2}
\end{gathered}
$$

onde $\boldsymbol{E}(\varepsilon)$ é a Esperança matemática de $\varepsilon$ e $\operatorname{Var}(\varepsilon)$ é a Variância de $\varepsilon$.

Caso a série temporal financeira se configure como um passeio aleatório, então o valor do desvio padrão é igual a $\sigma_{\varepsilon} t^{\frac{1}{2}}$. A estatística $R / S$ testa a hipótese nula do expoente da variável $t$ ser igual a $\frac{1}{2}$. Chamado de expoente $H$, homenagem de Mandelbrot a Hurst e ao matemático Ludwing Otto Hölder (82), pode-se definir o teste pela hipótese nula $H_{0}: H=\frac{1}{2}$ e pela hipótese alternativa $H_{0}: H \neq \frac{1}{2}$.

Mandelbrot (81) também desenvolveu um método para estimar o parâmetro $H$. Por ele, se rejeita a hipótese nula porque $H>\frac{1}{2}$ (caso de persistência) ou porque $H<\frac{1}{2}$ (caso de antipersitência).

Pode-se obter o método desenvolvido por Mandelbrot acompanhando a apresentação de Couillard (29) e abordado por Peters (106), conforme descrito a seguir.

Seja uma série temporal com $N$ observações para intervalos iguais de tempo. Divide-se as 
observações em $M$ subperíodos com o mesmo número de observações $t$, de tal forma que $M \times t=N$, define-se $I_{m}, m=1,2, \ldots, M$, como cada um dos $M$ subperíodos e $N_{k, m}$, $k=1,2, \ldots, t$, como cada elemento de determinado subperíodo. Com isso pode-se definir a média $\mu_{I_{m}}$ e o desvio padrão $S_{I_{m}}$ de cada subperíodo como:

$$
\begin{gathered}
\mu_{I_{m}}=\frac{1}{t} \sum_{k=1}^{n} N_{k, m} \\
S_{I_{m}}=\sqrt{\frac{1}{t} \sum_{k=1}^{n}\left(N_{k, m}-\mu_{I_{m}}\right)^{2}}
\end{gathered}
$$

A Partir dos valores das médias pode-se reconstruir a série original e obter uma série com $M$ sequências $I_{m}$ compostas, cada uma, por $t$ desvios acumulados em relação a $\mu_{I m}$. Estes desvios acumulados são definidos conforme:

$$
X_{k, m}=\sum_{k=1}^{n}\left(N_{k, m}-\mu_{I_{m}}\right),
$$

sendo a amplitude dos desvios médios acumulados em cada sequência $I_{m}$ é definida por:

$$
R_{I_{m}}=\max \left(X_{k, m}\right)-\min \left(X_{k, m}\right)
$$

Então a série com $M$ valores $R_{I_{m}}$ é normalizada dividindo estes valores de amplitude pelos seus correspondentes desvios padrões $S_{I_{m}}$. A média destes valores padronizados mantém a relação entre $H$ e $t$. Portanto, a estatística é definida como:

$$
\frac{R}{S}=\frac{1}{M} \sum_{m=1}^{M} \frac{R_{I_{m}}}{S_{I_{m}}}=c t^{H},
$$

sendo $c$ uma constante.

Para obter-se o valor de $H$ e testá-lo deve-se calcular uma série de estatísticas $R / S_{t}$ para diferentes valores de $t$, linearizar a igualdade $R / S_{t}=c t^{H}$ e, com isso, estimar o valor de $H$. Para linearizar a igualdade $R / S_{t}=c t^{H}$, basta aplicar o logaritmo:

$$
\log \left(\frac{R}{S}\right)=\log (c)+H \log (t)
$$

Com tal característica, o valor de $H$ pode ser estimado mediante uma regressão linear simples. Como $R_{I_{m}}$ é sempre maior ou igual a zero e $S_{I_{m}}$ é sempre maior que zero, o valor de $H$ terá limite inferior perto de zero, dependendo do valor de $c$. Como $R_{I_{m}} / S_{I_{m}}$ são somatórios de $t$ valores normalizados, seu valor máximo tende a $t$ e, portanto, o valor máximo de $H$ tende a 1 , dependendo de $c$. 
A questão na realização deste teste é definir o tamanho dos subperíodos $I_{m}$ de forma a conservar, para cada valor de $R / S_{t}$ um número o mais próximo possível de variáveis. Também, os valores de $t$ devem ser preferencialmente divisores inteiros de $N$, ou números inteiros o mais próximo possível de algum divisor do tamanho da série, de forma que a quantidade de dados excluídos possa ser mínima.

\subsubsection{Método para Obtenção do Expoente de Hurst}

O método utilizado para o cálculo estimado do Expoente de Hurst $(H)$ por meio da análise $\mathrm{R} / \mathrm{S}$ segue a metodologia desenvolvida Mandelbrot e Wallis (85), baseada nos trabalhos de Hurst (62) (28).

O método aqui apresentado consiste na execução das subsequentes etapas, destacadas na apresentação de Couillard (29) e explorado por Peters (106):

- Seleção dos dados da série temporal, previamente executada para o ajuste mediante o modelo híbrido SVR-wavelet;

- Obtenção da média $\left(E_{m}\right)$ e o desvio padrão $\left(S_{m}\right)$ da subsérie $\left(Z_{i, m}\right)$;

- Normalização dos dados da subsérie $\left(Z_{i, m}\right)$ pela subtração da média das amostras $X_{i, m}=$ $Z_{i, m}-E_{m}$, para $i=1, \ldots, N$;

- Geração da série de tempo acumulativa $Y_{i, m}=\sum_{j=1}^{i} X_{j, m}$, para $i=1, \ldots, N$;

- Obtenção da variação $R_{m}=\max \left\{Y_{1, m}, \ldots, Y_{n, m}\right\}-\min \left\{Y_{1, m}, \ldots, Y_{n, m}\right\}$;

- Obtenção da estatística $\left(R_{m} / S_{m}\right)$;

- Obtenção da médio $(R / S)_{n}$ da estatística $\left(R_{m} / S_{m}\right)$ de todas as subséries de tamanho $n$.

Considerando-se que a Estatística $R / S$ assintoticamente segue a relação $(R / S)_{n} \approx c n^{H}$, o valor do expoente de Hurst $(H)$ pode ser calculado por meio de uma regressão linear simples: $\log (R / S)_{n}=\log c+H \log n$.

Se o processo for um movimento Browniano, $H$ tem de ser $\frac{1}{2}$; quando $H$ for persistente é então maior do que $\frac{1}{2}$; e quando for anti-persistente $H$ é menor do que $\frac{1}{2}$. Para uma tendência linear simples $H=1$ e para um ruído branco $H=0$. Portanto $H$ tem de estar entre 0 e 1 . 


\subsubsection{Teste de Significância do Expoente de Hurst}

Segundo Couillard e Davison (29) a maioria dos estudos falham na constatação de $H \neq \frac{1}{2}$ por não fornecerem um teste de significância. Assim, para este trabalho realiza-se o teste sugerido por Couillard e Davison, utilizando-se da estatística p-value $<0,001$. 


\section{METODOLOGIA}

Em um primeiro momento tem-se o desenvolvimento do modelo SVR-wavelet híbrido que incorpora modelos de wavelets e SVR, regressão por meio de Máquinas de Suporte Vetorial (SVM). A Figura 8 ilustra a proposta do modelo para predição de séries temporais por meio do modelo SVR-wavelet híbrido.

O próximo passo é efetuar a seleção e preparação do conjunto de ativos financeiros do tipo FOREX, obtidos a partir de uma base de conhecimento público (116). Segundo alguns autores, o tratamento de dados é considerado uma tarefa complexa, delicada e que possui fundamental relevância nos processos de análise de dados (45) (86). Esta tarefa é responsável pela eliminação de dados irrelevantes do conjunto de resultados a serem analisados que inclui desde a correção de dados até o ajuste da formatação dos dados a serem utilizados para os modelos de ajustes de séries temporais (55) (149).

Depois demonstra-se que as séries são apropriadas e favoráveis à utilização da estratégia SVR-wavelet híbrida. São realizados testes estatísticos como os de normalidade e de raiz unitária para se comprovar que as séries em estudo possuem distribuição não linear e também evidenciar o nível de correlação entre os períodos das séries por meio dos testes de correlação por meio do gráfico de dispersão.

A esse conjunto de ativos financeiros do tipo FOREX é aplicado o modelo proposto SVRwavelet em que uma parte dos dados é selecionada e separada como dados de treino e outra parte dos dados é selecionada e separada como dados de teste.

Em seguida efetua-se o ajuste deste conjunto de ativos financeiros do tipo FOREX segundo os modelos tradicionais ARFIMA, ARIMA, GARCH e o modelo SVR tradicional com Kernel.

Logo depois são efetuados testes quanto a precisão dos modelos ajustados (142) (94) (127), dos quais este trabalho destaca: Erro Quadrático Médio (MSE), Raiz Quadrada do Erro Quadrático Médio (RMSE), Erro Absoluto Médio (MAE), Erro Porcentual Médio (MPE), Erro Porcentual Absoluto Médio (MAPE), Erro Escalado Absoluto Médio (MASE), Autocorrelação de Erros para uma Defasagem (ACF1), Critério de Informação de Akaike (AIC), AIC de Segunda Ordem (AICc) e Critério de Informação Bayesiano (BIC).

Com a série ajustada pelos modelos tradicionais e pelo modelo SVR-wavelet realiza-se a previsão de alguns períodos à frente, comparando-se os resultados. Nesta etapa também se faz a verificação quando a aderência ao Expoente de Hurst, obtido antes e depois da previsão dos períodos à frente, por meio da estatística R/S.

Por último, desenvolve-se a análise dos resultados verificando-se também se o modelo proposto por este trabalho consegue superar a previsão efetuada pelos modelos tradicionais.

Com a completude dos testes é apresentada uma análise geral do trabalho onde se discorre 
acerca dos problemas enfrentados nos testes e sua aceitação frente aos modelos tradicionais.

A Figura 9 ilustra a metodologia adotada na predição de séries temporais por meio do modelo SVR-wavelet híbrido.
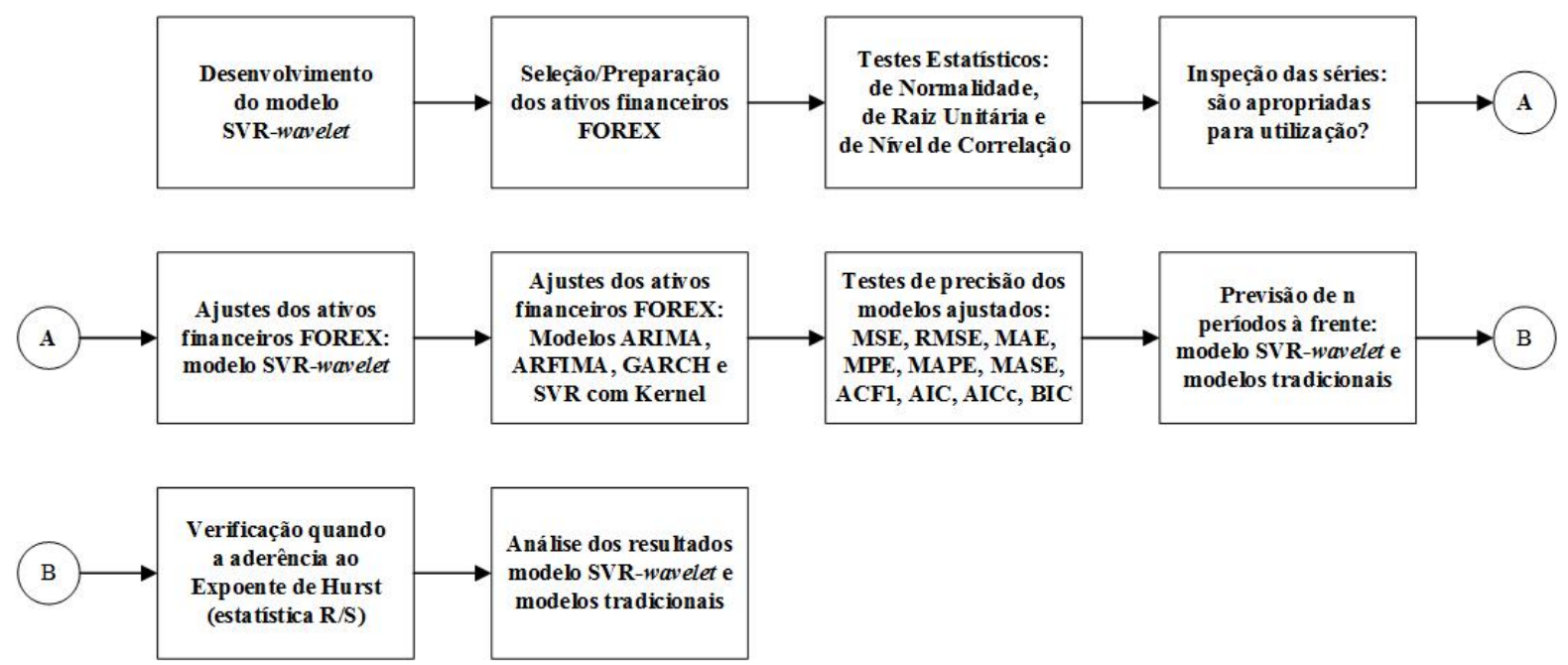

Figura 9 - Representação da metodologia adotada na predição de séries temporais por meio do modelo SVRwavelet híbrido. 


\section{AMBIENTE DE PROGRAMAÇÃO PARA ANÁLISE DE DADOS ESTATÍSTICOS E GRÁFICOS R}

Neste capítulo são apresentadas as bibliotecas e também os métodos utilizados na aplicação do modelo híbrido SVR-wavelet para previsão de séries temporais financeiras, valendo-se do ambiente de programação para análise de dados estatísticos e gráficos $R$ (47).

Embora exista uma lista suficientemente extensa de métodos que possivelmente possam ser utilizados para a aplicação do modelo proposto, percebeu-se que, durante a realização do modelo SVR-wavelet, elas não eram totalmente adequadas ou mesmo robustas no desenvolvimento do modelo híbrido proposto.

Com isso, uma solução composta, envolvendo o emprego da linguagem de programação $R$ e o uso de determinadas bibliotecas, fez-se necessária e se mostrou mais eficiente e consistente que aquela que emprega métodos nativos do ambiente $R$.

No conjunto de programas desenvolvidos para a realização do modelo proposto estão as métricas adotadas por este trabalho, MSE, RMSE, MAE, MPE, MAPE, MASE, ACF1, AIC, AICc e BIC, incluindo o método desenvolvido por Mandelbrot (de acordo com a apresentação de Couillard) para determinação do Expoente de Hurst (R) por meio da Análise R/S (29), o fluxo de execução das bibliotecas e métodos em $R$, tal como a preparação dos dados FOREX selecionados.

A lista completa dos programas desenvolvidos em $R$ é apresentada como anexo a este trabalho.

\subsection{USO DO MÉTODO SVM}

O método svm é utilizado para treinar a Máquina de Suporte Vetorial com o objetivo de realizar regressões não-lineares estimando um valor condicional não esperado (89). Em $R$, este método possui os seguintes parâmetros (12) (90):

svm (formula, data, scale, type, kernel, gamma, cost, tolerance, epsilon, shrinking, fitted)

em que formula é a Notação de Fórmula Estatística em $R$ do modelo a ser ajustado; data contém as variáveis no modelo; scale é um vetor lógico e indica que os dados (as variáveis $x$ e $y$ ) são tratados com média zero, $\mu=0$, e variância unitária, $\sigma^{2}=1$; type é o tipo do SVM utilizado, i.e. regressão, e contem o valor eps - regression; kernel é o kernel usado no treinamento e previsão, e.g. radial; gamma é o parâmetro gama empregado em alguns kernels como o kernel radial (12); cost é a constante $C$ do termo de regularização de $\mathcal{L}$ agrange, 
também aplicado em alguns kernels como o radial (12); tolerance é a tolerância do critério de término e frequentemente é 0,001 (12); epsilon é a função de perda $\varepsilon$ - insensível, loss function, geralmente 0,1 (12); shrinking é o indicador lógico para o uso de funções de reduções e fitted é o valor lógico indicando se os valores ajustados devem ser calculados e incluídos no modelo.

Para o escopo deste trabalho somente são utilizados os parâmetros apresentados no parágrafo anterior. Contudo, segundo o manual de utilização da biblioteca e1071 (90), existem muitos outros parâmetros, que podem ser melhores estudados em pesquisas futuras.

A Notação de Fórmula Estatística em $R$ é ampla e com muitas variantes (54). Contudo, uma breve explicação se faz necessária. formula é a descrição simbólica do modelo a ser ajustado e seu formato básico é: variável de resposta $\sim$ variáveis preditoras, onde $\sim$ é lido como é modelado como uma função das. Consequentemente, para se efetuar a análise de uma regressão básica, o formato da formula seria: $Y \sim X$.

Então, pode-se ajustar um modelo por meio do svm, regredindo $Y$ em $X$ como modelo $\leftarrow$ $\operatorname{svm}(Y \sim X)$, onde $X$ é a variável preditora e $Y$ é a variável de resposta. Valendo-se da notação matemática, este modelo de regressão é apresentado como $Y_{i}=\beta_{0}+\beta_{1} X_{i}+\omega_{i}$.

Variáveis explicativas adicionais podem ser incluídas usando o símbolo + . Para adicionar outra variável preditora $Z$, a formula é expressa como $Y \sim X+Z$; em $R$ é apresentada como modelo $\leftarrow \operatorname{svm}(Y \sim X+Z)$, produzindo uma regressão múltipla com dois preditores. A correspondente notação matemática é: $Y_{i}=\beta_{0}+\beta_{1} X_{i}+\beta_{2} Z_{i}+\omega_{i}$.

Em $R$, data é usado para armazenar tabelas de dados. É uma lista de vetores de igual comprimento. Se $X$ e $Z$ forem componentes deste tipo de estrutura de dados, a expressão modelo $\leftarrow \operatorname{svm}(Y \sim X+Z)$ pode ser simplificada pela expressão: modelo $\leftarrow$ svm $(Y \sim$., data $=D)$, onde o símbolo . indica a inclusão de todas as variáveis, no caso $X$ e $Y$, contidas na lista de vetores $D$.

Nos exemplos expostos na Notação de Fórmula Estatística em $R$ optou-se simplificar o uso do método svm somente como uma forma de facilitar a explicação do uso da notação. Todavia, para os testes por meio do método svm, todos os parâmetros previamente descritos são empregados.

\subsection{USO DO MÉTODO PREDICT}

O método predict é empregado para predizer valores futuros baseando-se em modelos previamente treinados pelo método svm (89). Em $R$ este método possui os seguintes parâmetros (12) (90): 
em que object é o modelo criado pelo método svm e newdata é a matriz que contém os novos dados de entrada. À vista disso, usando o método predict, um exemplo de previsão empregando-se parte dos dados de entrada, seria: pred $\leftarrow$ predict (modelo, $Y[,-100]$ ).

Um terceiro método, tune, pode ser empregado para se regular os parâmetros de entrada do método svm. Também disponível para uso na biblioteca e1071, o método tune ajusta hiperparâmetros de métodos estatísticos usando procedimentos de pesquisa sobre os parâmetros fornecidos.

\subsection{USO DOS MÉTODOS WD E WR}

Existem disponíveis algumas possibilidades de decomposição em coeficientes wavelet, das quais duas são mais frequentemente aplicadas.

A primeira delas é por meio da execução da biblioteca wavethresh (98). Dentre muitos métodos, esta biblioteca dispõe dos métodos $w d$, para efetuar a Transformada wavelet, e $w r$, para realizar o cálculo de sua Transformada Inversa.

Embora encontram-se disponíveis alguns parâmetros para a aplicação do método $w d$, somente os parâmetros data e family são de fato importantes para este método, em conformidade com o formato (98):

$$
w d(\text { data, family) }
$$

em que data é um vetor de potência de 2 contendo os dados a serem decompostos e family define a família de wavelet a ser empregada nos cálculos da Transformada wavelet.

As possíveis famílias para o parâmetro family são DaubExPhase e DaubLeAsymm para as wavelets Daubechies, Coiflets para as wavelets Coiflets, Lawton para as wavelets Lawton, LittlewoodPaley para as wavelets Littlewood - Paley e LinaMayrand para as wavelets Lina - Mayrand - Daubechies (98).

Sua inversa, o método $w r$, tem como parâmetro a Transformada wavelet (98):

$$
w r(w d)
$$

Diante disso, um exemplo da aplicação da Transformada wavelet e de sua Transformada inversa, empregando-se os dados de entrada como um vetor de potência 2 e recorrendo-se do uso da wavelet Daubechies DaubExPhase, seria: wavelet $\leftarrow$ wd (vetor.p2, family $=$ "DaubExPhase") e vetor.p $2 \leftarrow$ wr (wavelet). 


\subsection{USO DOS MÉTODOS WAVMODWT E RECONSTRUCT}

Outra possibilidade para decomposição em coeficientes wavelet, valendo-se do ambiente de programação para análise de dados estatísticos e gráficos $R$ (47), é através do uso da biblioteca wmtsa (23). Esta biblioteca possui os métodos wavMODWT, para efetuar a Transformada wavelet Discreta de Máxima Sobreposição (MODWT), e reconstruct, para realizar o cálculo de sua Transformada inversa (23), escopo do estudo deste trabalho.

Apesar de haver múltiplos parâmetros para a aplicação do método wav $M O D W T$, não mais que quatro parâmetros são necessários e significativos para o uso deste método, de acordo com o modelo (23):

wavMODWT (data, wavelet $="$ family", n.level $=$ integer, keep.series $=$ boolean $)$

em que data é o vetor contendo a série temporal, wavelet qualifica a família wavelet, n.level estabelece o número de níveis de decomposição e keep.series, com os valores lógicos $T R U E$ ou FALSE, indicando a preservação da série original como um objeto de saída.

Para este método as prováveis famílias wavelets aplicáveis são (111) (30):

- daublet: "haar", "d2", "d4", "d6", "d8", "d10", "d12", "d14", "d16", "d18" e "d20";

• symmlet: "s2", "s4", "s6", "s8", "s10", "s12", "s14", "s16", "s $18 "$ e "s20";

- Best Localized (BL): "l2", "l4", "l6", "l14", "l18" e "l20"; e

- coiflet: "c6", "c12", "c18", "c24" e "c30".

em que o número de índice refere-se ao número de coeficientes.

Sua inversa, calculada pelo método reconstruct, apenas possui um parâmetro, a Transformada wavelet gerada por uma das classes wavTransform da biblioteca wmtsa, no caso wavMODWT (23):

$$
\text { reconstruct (wavMODWT) }
$$

Segundo Cornish (26), o MODWT oferece vantagens sobre a Transformada de Wavelet Discreta (DWT). A redundância do MODWT facilita o alinhamento dos componentes de frequência e escala, decompostos em cada nível da série temporal original, permitindo uma comparação imediata entre a série e sua decomposição. As Análises de Variância (ANOVA), procedentes do uso do MODWT, não são influenciadas pelo deslocamento, mesmo circular, das séries temporais de entrada, enquanto que os valores obtidos utilizando-se o DWT dependem do ponto de partida da série. Finalmente, a redundância dos coeficientes da wavelet MODWT 
aumenta os Graus de Liberdade Efetivos (EDOF) em cada escala e, consequentemente, diminui a variância de certas estimativas estatísticas baseadas em wavelets. Pela característica do MODWT em conservar a energia, ele também é adequado para analisar a dependência de escala da variabilidade em estudos de ANOVA.

Isso posto, um exemplo da aplicação da MODWT e sua Transformada inversa, para decomposição em coeficientes wavelet através da utilização dos métodos wavMODWT e reconstruct, valendo-se dos dados de entrada e servindo-se do uso da wavelet daublet haar ou $d 2$, seria: wavelet $\leftarrow$ wavMODWT(data, wavelet $=$ "haar", n.level $=5$, keep.series $=T R U E)$ e data $\leftarrow$ reconstruc (wavelet).

\subsection{USO DO MÉTODO THRESHOLD}

Para efetuar a eliminação de ruídos, por meio de técnicas de denoising ou wavelet shrinkage, - ambiente de programação para análise de dados estatísticos e gráficos $R$ (47) dispõe de algumas bibliotecas.

Os métodos denoise.dwt.2d e denoise.modwt.2d, encontrados na biblioteca waveslim (46), são adequados no tratamento de ruídos contidos em imagens.

Já a biblioteca rwt (121), com seu método denoise.dwt, e a biblioteca wavethresh (99), com seu método threshold, se manifestam mais apropriadas no tratamento de ruídos contidos em séries financeiras. Contudo, efetuando-se a reconstrução da série original e comparando os erros quadráticos médios (MSE) gerados, o método threshold se mostrou mais aderente às séries financeiras do que o método denoise.dwt.

Dessa forma, este trabalho emprega o método threshold, acessível por intermédio da biblioteca wavethresh, para a eliminação de ruídos, possivelmente contidos nas séries financeiras do tipo FOREX, por meio de técnicas de denoising ou wavelet shrinkage, conforme os parâmetros (100):

$$
\text { threshold (object) }
$$

em que object é a Transformada de Wavelet Discreta (DWT) da série original.

\subsection{USO DOS MÉTODOS SARIMA E SARIMA.FOR}

Em $R$, servindo-se da biblioteca astsa (127), é possível ajustar séries temporais ao modelo ARIMA mediante à aplicação do método sarima, bem como realizar previsões $n$ passos à frente de séries temporais através de seu método sarima.for. 
Dentro dos possíveis parâmetros utilizáveis em $R$ o método sarima dispõe de quatro principais parâmetros (127):

$$
\text { sarima (object, } p, d, q)
$$

em que object é a série original a ser modelada e $p, d, q$ são os parâmetros de ordem do modelo ARIMA.

Sendo assim, operando o método sarima, um exemplo de ajuste empregando-se dos dados de entrada seria prices.fit.sarima $\leftarrow$ sarima (prices.ts, 1, 0, 1), onde prices.ts é a série original a ser modelada através do modelo ARIMA, e 1, 0, 1 é a ordem do modelo.

Há uma variedade de parâmetros acessíveis em $R$ para o método sarima.for. Apesar disso, cinco parâmetros são ademais relevantes (127):

$$
\text { sarima.for (object, n.ahead, } p, d, q \text { ) }
$$

em que object é a série temporal inicial, n.ahead é o número de períodos para previsão e $p, d, q$ são os parâmetros de ordem do modelo ARIMA. Pois então, sarima.for, um exemplo de previsão de $n$ passos à frente empregando-se a série original, seria prices.pred.sarima $\leftarrow$ sarima.for (prices.ts, n.ahead $=1,1,0,1$ ), no qual prices.pred.sarima é o objeto contendo os $n$-ésimos pontos preditos, $n$ passos à frente, prices.ts é o vetor contendo a série temporal originária, e n.ahead $=1$ é o número de períodos para previsão, que neste exemplo é $1, h=1$, um passo à frente.

\subsection{USO DOS MÉTODOS ARFIMA E FORECAST}

Valendo-se do ambiente de programação para análise de dados estatísticos e gráficos $R$ (47), provida da biblioteca forecast (63), é possível ajustar séries temporais mediante à aplicação do método arfima bem como realizar previsões $n$ passos à frente de séries temporais ou modelos de séries temporais, através de seu método forecast.

Dentro dos variados parâmetros disponíveis em $R$ o método ar fima dispõe de três principais parâmetros (63):

$$
\text { arfirma (object, drange, estim) }
$$

em que object é a série original a ser modelada, drange permite que sejam considerados os valores $d$, tendo como padrão drange $=c(0,0.5)$ o que garante ajustes de modelos estacionários, e estim $=c($ "mle") para ajustes de modelos através da Estimativa por Máxima Verossimilhança (MLE). 
À vista disso, um exemplo de ajuste empregando-se dos dados de entrada, seria:

prices.fit.arfima $\leftarrow$ arfima (prices.ts, drange $=c(0,0.5)$, estim $=c(" m l e "))$.

Entre os diversos parâmetros acessíveis em $R$ o método forecast possui dois parâmetros relevantes (63):

$$
\text { forecast (object, } h \text { ) }
$$

em que object é a série temporal ou o modelo para o qual a previsão é requerida e $h$ é o número de períodos para previsão. Diante disso, um exemplo de previsão de $n$ passos à frente empregando-se a série original seria prices.fcast.arfima $\leftarrow$ forecast (prices.fit.arfima, $h=1$ ), no qual prices.fcast.arfima é o objeto contendo os $n$-ésimos pontos preditos, $n$ passos à frente, prices. fit.arfima é o vetor contendo o modelo de série temporal e $h$ é o número de períodos para previsão, $(h=1)$, um passo à frente.

\subsection{USO DO MÉTODO GARCHFIT}

Com suas bibliotecas em $R$, em particular a biblioteca fGarch (142), é possível ajustar as séries temporais mediante a aplicação do método garchFit, bem como realizar previsões $n$ passos à frente de séries temporais através do método predict, especificado na última seção.

Em $R$, o método fGarch possui os seguintes parâmetros relevantes para este trabalho:

$$
\operatorname{garchFit}(\text { formula }=\sim \operatorname{garch}(1,1), \text { data }=\text { data object }, \text { trace }=\text { boolean })
$$

em que formula é a Notação de Fórmula Estatística em $R$ do modelo a ser ajustado, data contém as variáveis no modelo e trace possibilita a impressão imediata de dados estatísticos do modelo.

Um exemplo de ajuste por meio do método garchFit aplicado aos dados de entrada, seria prices.fit.garch $\leftarrow$ garchFit (prices $\sim \operatorname{garch}(1,1)$, data $=$ prices.data, trace $=$ FALSE), em que prices e prices.data contém a série original a ser modelada através do modelo GARCH, e trace $=F A L S E$ desabilita a imediata impressão de dados estatísticos do modelo.

Usando o método forecast, um exemplo de previsão de $n$ passos à frente empregandose a série original, seria prices.pred.garch $\leftarrow$ forecast (prices.fit.garch, $h=1$ ), no qual prices.pred.garch é o objeto contendo os $n$-ésimos pontos preditos, $n$ passos à frente, prices.fit.garch é o vetor contendo o modelo de série temporal, e $h$ é o número de períodos para previsão, neste exemplo é um passo à frente. 


\section{APLICAÇÃO DO MODELO SVR-WAVELET}

Apresentação e aplicação do método proposto conforme a metodologia apresentada neste trabalho.

\subsection{SELEÇÃO E CARACTERIZAÇÃO DAS SÉRIES TEMPORAIS DO TIPO FOREX}

O mercado de câmbio FOREX, Foreign Exchange Market (116), é um mercado global para a negociação descentralizada de moedas. Em termos de volume de negociação, é de longe o maior mercado do mundo. Os principais participantes deste mercado são os maiores bancos internacionais. São eles os responsáveis pela definição dos valores relativos das diferentes moedas.

A atual estrutura do mercado FOREX evoluiu a partir das taxas de câmbio flutuantes. Os pares de moedas mais negociados no mercado à vista FOREX hoje são (114): 27\% EUR-USD, 13\% USD-JPY e 12\% GBP-USD. Um fato importante é que desde a criação da moeda única europeia, em janeiro de 1999, o comércio de Euro cresceu consideravelmente. O mercado FOREX negocia cinco dias por semana sem parar e é influenciado por muitos fatores econômicos e geopolíticos, como a balança de pagamentos em cada país, a balança comercial, as políticas fiscal e monetária, a estabilidade política e a solidez de crédito dos governos. Os principais centros FOREX são Nova York, Londres, Singapura, Tóquio e Hong Kong.

Para este trabalho foram selecionadas cinco séries temporais financeiras do tipo FOREX. São utilizadas as seguintes relações entre moedas globalizadas:

- Dólar Australiano (AUD) e Yen Japonês (JPY);

- Franco Suíço (CHF) e Yen Japonês (JPY);

- Euro (EUR) e Franco Suíço (CHF);

- Euro (EUR) e Yen Japonês (JPY); e

- Libra Britânica (GBP) e Yen Japonês (JPY).

Os ativos FOREX listados são séries temporais que compõem históricos de preços de moedas entre o período de 01/01/2003 a 30/12/2014, com periodicidade de 1 em 1 minuto, obtidos a partir da base de conhecimento público (116).

É criado um framework, desenvolvido em Visual Studio .NET, Framework 4.6.2 (59), 64bits, Ambiente Windows, para geração e seleção de três novas periodicidades para execução 
dos testes deste trabalho, totalizando uma quantidade ao redor de 3000 valores por série gerada e selecionada, a saber:

- Série temporal com dados selecionados com intervalos de 1 em 1 dia;

- Série temporal com dados selecionados com intervalos de 1 em 1 hora; e

- Série temporal com dados selecionados com intervalos de 15 em 15 minutos, conforme fontes de programação anexos a este trabalho.

Com relação aos períodos destacados das séries, obtidos a partir da base de conhecimento público (116), originalmente com periodicidade de 1 em 1 minuto, são efetuadas seleções aleatórias de períodos contínuos de dados para os intervalos de 1 em 1 hora, de 15 em 15 minutos e para os intervalos de $1 \mathrm{em} 1 \mathrm{dia}$. Este procedimento tem por objetivo garantir que o método adotado neste trabalho possa ser testado e validado com imparcialidade contra períodos distintos de datas.

A partir daí, as séries de dados temporais são tratadas para eliminação de dados irrelevantes do conjunto de resultados a serem processados, o que inclui desde a correção de dados até o ajuste da formatação dos dados para os algoritmos de mineração de dados que serão utilizados, representando a fase principal do KDD (Knowledge Discovery in Databases) (79).

Para tratamento do conjunto de dados dos ativos financeiros do tipo FOREX, com suporte as fases do KDD - Descoberta de Conhecimento em Bases de Dados, assegurando assim a qualidade, completude, veracidade e integridade dos fatos por eles representados, é utilizado uma função chamada de Tunelamento de Preços, desenvolvida usando-se o ambiente de programação para análise de dados estatísticos e gráficos $R$ (47), versão 3.3 .2 de 31/10/2016, utilizando a plataforma mac $O S$ (C), processador $3.3 \mathrm{GHz}$ Core $i 7$, com 16 GB de memória.

O algoritmo desta função tem por objetivo verificar distorções nos preços de um dado período da série, por meio de uma variação porcentual $\Delta P_{t}$ do valor atual do preço $P_{t}$ em relação ao preço anterior $P_{t-1}$. Normalmente esta variação porcentual $\Delta P_{t}$, adotada nos mercados financeiros, não é superior a $5 \%$ do preço atual $P_{t}$ tanto para cima como para baixo em relação ao preço anterior $P_{t-1}$.

Assim a variação $\Delta P_{t}$, respeitando-se o túnel de preços, é expressa por:

$$
0.95<\Delta P_{t}<1.05
$$

Se o preço atual $P_{t}$ estiver fora to túnel de preços o algoritmo gera um preço $P_{t}$ aleatório, a partir do preço anterior $P_{t-1}$, respeitando os limites do túnel de preços. 
Foram amostrados 3009 pontos por série histórica dos preços do ativo FOREX, gerados com periodicidades de $1 \mathrm{em} 1 \mathrm{dia}, 1 \mathrm{em} 1$ hora e $15 \mathrm{em} 15$ minutos, respectivamente para os períodos de: $01 / 01 / 2003$ a 30/12/2014, 07/11/2012 a 06/05/2013 e 15/03/2012 a 29/04/2012.

A Figura 10 apresenta os gráficos contendo as séries históricas dos preços do ativo FOREX AUD-JPY com periodicidades de 15 minutos, 1 hora e 1 dia, com a aplicação de técnicas de denoising ou wavelet shrinkage para eliminação de ruídos contidos na série. Somente os últimos 2048 pontos são amostrados.

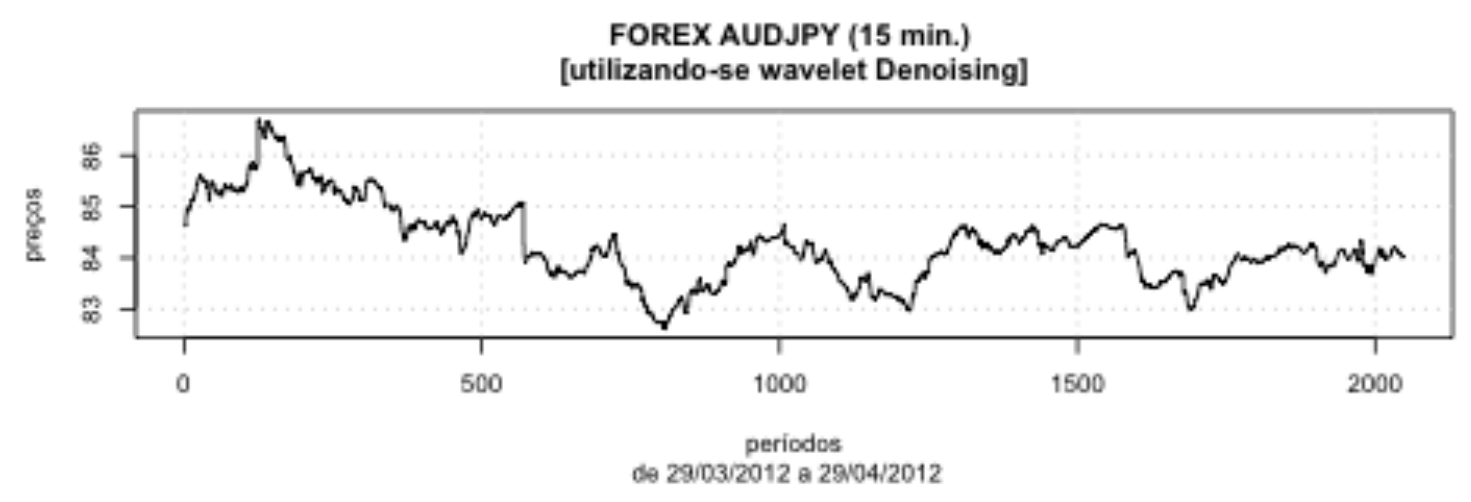

FOREX AUDJPY ( 1 dia)

[utilizando-se wavelet Denoising]

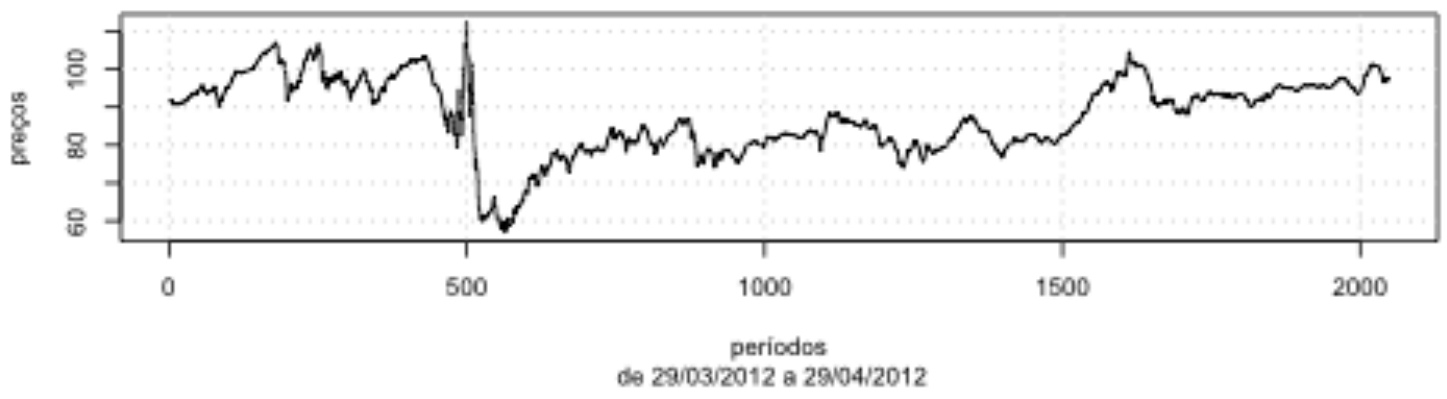

FOREX AUDJPY (1 hora)

[utilizando-se wavelet Denoising]

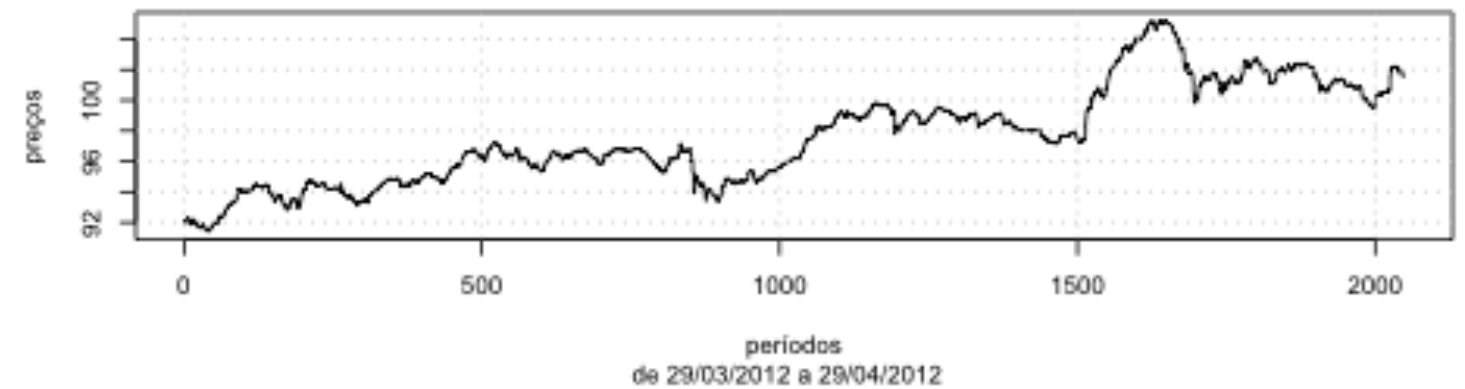

Figura 10 - Gráficos contendo as séries históricas dos preços do ativo FOREX AUD-JPY com periodicidades de 15 minutos, 1 hora e 1 dia, com a aplicação de técnicas de denoising ou wavelet shrinkage para eliminação de ruídos contidos na série. Somente os últimos 2048 pontos são amostrados. 


\subsection{TESTES DE NORMALIDADE, RAIZ UNITÁRIA E DE NÃO-LINEARIDADE}

Anteriormente a construção de qualquer modelo, os dados devem ser avaliados para se constatar se as séries a serem estudadas são apropriadas para utilização. Para o uso desses conjuntos de dados do tipo FOREX, faz-se necessário testar suas características de não-linearidade e não-estacionariedade aplicando-se alguns testes estatísticos, como o teste de Anderson-Darlin, o teste de Smirnov-Cramer-Von Mises, o teste de Lilliefors (Kolmogorov-Smirnov), o teste de Qui-quadrado de Pearson, o teste de Mickey-Fuller e o teste de Elliott-Rothenberg. São também apresentados outros testes estatísticos como AIC, AICc e o BIC, bem como testes de Raiz Unitária e de Correlação.

Foram usadas as séries temporais que compõem históricos de preços de moedas, um conjunto de ativos financeiros de taxas de câmbio, obtidos a partir de uma base de conhecimento público (116), entre o período de 01/01/2003 a 30/12/2014, para as relações entre as moedas: Dólar Australiano (AUD) e o Yen Japonês (JPY), Franco Suíço (CHF) e o Yen Japonês (JPY), Euro (EUR) e o Franco Suíço (CHF), Euro (EUR) e o Yen Japonês (JPY), e a Libra Britânica (GBP) e o Yen Japonês (JPY), com periodicidades de 15 minutos, 1 hora e 1 dia.

Os testes foram efetuados usando-se o ambiente de programação para análise de dados estatísticos e gráficos $R$ (47), versão 3.3.2 de 31/10/2016, utilizando a plataforma mac OS (C), processador $3.3 \mathrm{GHz}$ Core $i 7$, com $16 \mathrm{~GB}$ de memória e suas bibliotecas nortest, astsa e tseries para os testes de Normalidade. A biblioteca tseries também foi utilizada para os testes de Raiz Unitária e de Não-Linearidade.

Para a montagem e execução dos testes foram adotados alguns passos de acordo com que está exibido na Figura 11 com o macro fluxograma das principais tarefas no processo de montagem e execução dos testes.
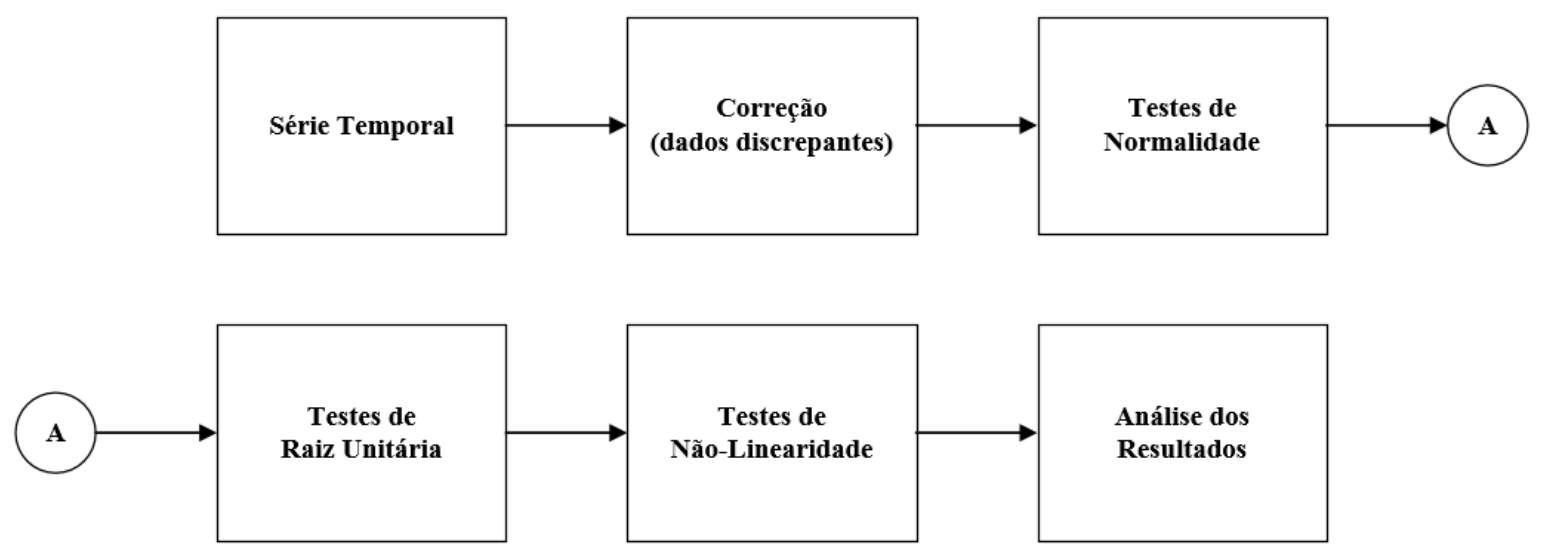

Figura 11 - Macro fluxograma das principais tarefas no processo de montagem e execução dos testes. 
São apresentados os resultados para os testes de Normalidade para uma distribuição não linear, conforme métodos expostos neste trabalho.

Primeiramente empregando-se os métodos para testes de Normalidade, depois operandose com os métodos para testes de Raiz Unitária e na sequência efetuando-se os testes de Não-Linearidade.

Dessa forma, a Tabela 2 sumariza os resultados encontrados, empregando-se os métodos Anderson-Darling (AD), Cramer-von Mises (CVM), (Lilliefors) Kolmogorov-Smirnov (LKS), Pearson chi-square(PCS), Shapiro-Francia (SF), Shapiro-Wilk (SW) e Jarque-Bera (JB) para testes de Normalidade.

Observa-se pela Tabela 2 que para as séries AUD-JPY, CHF-JPY, EUR-CHF, EUR-JPY e GBP-JPY, com as periodicidades de 15 minutos, 1 hora e 1 dia, o p-value experimentado é muito pequeno, $p<0,01$, representando então uma evidência muito forte contra $H_{0}$, o que leva a sua rejeição e a aceitação da hipótese alternativa. Decorre-se assim que as séries apresentadas para estudo deste relatório possuem uma distribuição não linear.

Tabela 2 - Resultados dos p-values dos métodos para testes de Normalidade.

\begin{tabular}{|c|c|c|c|c|c|c|c|}
\hline Série/Métodos & AD & CVM & LKS & PCS & SF & SW & JB \\
\hline AUDJPY $15 \mathrm{~min}$. & $<2.2 \mathrm{e}-16$ & $<7.37 \mathrm{e}-10$ & $<2.2 \mathrm{e}-16$ & $<2.2 \mathrm{e}-16$ & $1.544 \mathrm{e}-12$ & $4.145 \mathrm{e}-14$ & $3.037 \mathrm{e}-10$ \\
\hline AUDJPY 1hora & $<2.2 \mathrm{e}-16$ & $<7.37 \mathrm{e}-10$ & $<2.2 \mathrm{e}-16$ & $<2.2 \mathrm{e}-16$ & $4.552 \mathrm{e}-13$ & $1.089 \mathrm{e}-14$ & $1.638 \mathrm{e}-10$ \\
\hline AUDJPY $1 \mathrm{dia}$ & $<2.2 \mathrm{e}-16$ & $<7.37 \mathrm{e}-10$ & $<2.2 \mathrm{e}-16$ & $<2.2 \mathrm{e}-16$ & $6.003 e-14$ & $1.182 \mathrm{e}-15$ & $2.492 \mathrm{e}-12$ \\
\hline CHFJPY 15 min. & $<2.2 \mathrm{e}-16$ & $<7.37 \mathrm{e}-10$ & $<2.2 \mathrm{e}-16$ & $<2.2 \mathrm{e}-16$ & $6.402 \mathrm{e}-13$ & $2.492 \mathrm{e}-14$ & 0.0001535 \\
\hline CHFJPY 1 hora & $<2.2 \mathrm{e}-16$ & $<7.37 \mathrm{e}-10$ & $<2.2 \mathrm{e}-16$ & $<2.2 \mathrm{e}-16$ & $4.173 \mathrm{e}-10$ & $2.699 \mathrm{e}-11$ & $1.994 \mathrm{e}-06$ \\
\hline CHFJPY 1 dia & $<2.2 \mathrm{e}-16$ & $<7.37 \mathrm{e}-10$ & $<2.2 \mathrm{e}-16$ & $<2.2 \mathrm{e}-16$ & $<2.2 \mathrm{e}-16$ & $<2.2 \mathrm{e}-16$ & $<2.2 \mathrm{e}-16$ \\
\hline EURCHF $15 \mathrm{~min}$. & $<2.2 \mathrm{e}-16$ & $<7.37 \mathrm{e}-10$ & $<2.2 \mathrm{e}-16$ & $<2.2 \mathrm{e}-16$ & $<2.2 \mathrm{e}-16$ & $<2.2 \mathrm{e}-16$ & $<2.2 \mathrm{e}-16$ \\
\hline EURCHF 1 hora & $<2.2 \mathrm{e}-16$ & $<7.37 \mathrm{e}-10$ & $<2.2 \mathrm{e}-16$ & $<2.2 \mathrm{e}-16$ & $<2.2 \mathrm{e}-16$ & $<2.2 \mathrm{e}-16$ & $<2.2 \mathrm{e}-16$ \\
\hline EURCHF 1 dia & $<2.2 \mathrm{e}-16$ & $<7.37 \mathrm{e}-10$ & $<2.2 \mathrm{e}-16$ & $<2.2 \mathrm{e}-16$ & $<2.2 \mathrm{e}-16$ & $<2.2 \mathrm{e}-16$ & $<2.2 \mathrm{e}-16$ \\
\hline EURJPY 15 min. & $<2.2 \mathrm{e}-16$ & $<7.37 \mathrm{e}-10$ & $<2.2 \mathrm{e}-16$ & $<2.2 \mathrm{e}-16$ & $1.224 \mathrm{e}-14$ & $<2.2 \mathrm{e}-16$ & $6.162 \mathrm{e}-14$ \\
\hline EURJPY 1 hora & $<2.2 \mathrm{e}-16$ & $<7.37 \mathrm{e}-10$ & $<2.2 \mathrm{e}-16$ & $<2.2 \mathrm{e}-16$ & $2.222 \mathrm{e}-14$ & $3.651 \mathrm{e}-16$ & $6.078 \mathrm{e}-06$ \\
\hline EURJPY 1 dia & $<2.2 \mathrm{e}-16$ & $<7.37 \mathrm{e}-10$ & $<2.2 \mathrm{e}-16$ & $<2.2 \mathrm{e}-16$ & $<2.2 \mathrm{e}-16$ & $<2.2 \mathrm{e}-16$ & $<2.2 \mathrm{e}-16$ \\
\hline GBPJPY $15 \mathrm{~min}$. & $<2.2 \mathrm{e}-16$ & $<7.37 \mathrm{e}-10$ & $<2.2 \mathrm{e}-16$ & $<2.2 \mathrm{e}-16$ & $<2.2 \mathrm{e}-16$ & $<2.2 \mathrm{e}-16$ & $<2.2 \mathrm{e}-16$ \\
\hline GBPJPY 1 hora & $<2.2 \mathrm{e}-16$ & $<7.37 \mathrm{e}-10$ & $<2.2 \mathrm{e}-16$ & $<2.2 \mathrm{e}-16$ & $8.706 \mathrm{e}-14$ & $1.893 \mathrm{e}-15$ & $1.514 \mathrm{e}-07$ \\
\hline GBPJPY 1 dia & $<2.2 \mathrm{e}-16$ & $<7.37 \mathrm{e}-10$ & $<2.2 \mathrm{e}-16$ & $<2.2 \mathrm{e}-16$ & $<2.2 \mathrm{e}-16$ & $<2.2 \mathrm{e}-16$ & $<2.2 \mathrm{e}-16$ \\
\hline
\end{tabular}

Depois foram empregados os métodos para testes de Raiz Unitária Augmented Dickey-Fuller (ADF), Phillips-Perron (PP) e KPSS. Os resultados dos testes de Raiz Unitária são exibidos na Tabela 3 . 
Constata-se pela Tabela 3 que para as séries AUD-JPY, CHF-JPY, EUR-CHF, EUR-JPY e GBP-JPY, com as periodicidades de 15 minutos, 1 hora e 1 dia, os p-values experimentados são de $0,10<p$, configurando-se como pouca ou nenhuma evidência real contra $H_{0}$, exceto pela métrica KPSS onde os p-values estão muito mais próximos daqueles caracterizados como uma evidência moderada contra $H_{0}, 0,01 \leq p<0,05$, o que também, como nas duas outras métricas, ADF e PP, leva a sua aceitação e a rejeição da hipótese alternativa de que as séries são estacionárias. Dessa maneira, sucede-se que as séries apresentadas para estudo deste relatório possuem uma distribuição não estacionária.

Tabela 3 - Resultados dos p-values dos métodos para testes de Raiz Unitária.

\begin{tabular}{|l|l|l|l|}
\hline Série/Métodos & ADF & \multicolumn{1}{|c|}{ PP } & KPSS \\
\hline \hline AUDJPY 15 min. & 0.2653 & 0.3947 & 0.01 \\
\hline AUDJPY 1 hora & 0.4905 & 0.6681 & 0.01 \\
\hline AUDJPY 1 dia & 0.2443 & 0.3216 & 0.01 \\
\hline CHFJPY 15 min. & 0.4801 & 0.5153 & 0.01 \\
\hline CHFJPY 1 hora & 0.5467 & 0.7296 & 0.01 \\
\hline CHFJPY 1 dia & 0.6391 & 0.7691 & 0.01 \\
\hline EURCHF 15 min. & 0.4647 & 0.01 & 0.01 \\
\hline EURCHF 1 hora & 0.7099 & 0.2396 & 0.01 \\
\hline EURCHF 1 dia & 0.1756 & 0.02661 & 0.01 \\
\hline EURJPY 15 min. & 0.1589 & 0.0727 & 0.01 \\
\hline EURJPY 1 hora & 0.2651 & 0.4253 & 0.01 \\
\hline EURJPY 1 dia & 0.7185 & 0.8215 & 0.01 \\
\hline GBPJPY 15 min. & 0.6029 & 0.5828 & 0.01 \\
\hline GBPJPY 1 hora & 0.4999 & 0.6461 & 0.01 \\
\hline GBPJPY 1 dia & 0.6409 & 0.716 & 0.01 \\
\hline
\end{tabular}

Em seguida foram aplicados os métodos para testes de Não-Linearidade Terasvirta Neural Network (TNN) e White Neural Network (WNN). Os resultados dos testes de Não-Linearidade são retratados na Tabela 4 .

Nota-se pela Tabela 4 que para as séries AUD-JPY, CHF-JPY, EUR-CHF, EUR-JPY e GBP-JPY, com as periodicidades de 15 minutos, 1 hora e 1 dia, os p-values experimentados são de $0,10<p$, configurando-se como pouca ou nenhuma evidência real contra $H_{0}$, o que induz na sua aceitação e na rejeição da hipótese alternativa. À vista disso, verifica-se que as séries identificadas no estudo deste relatório se mostram claramente não lineares. 
Tabela 4 - Resultados dos p-values dos métodos para testes de Não-Linearidade.

\begin{tabular}{|l|l|l|}
\hline Série/Métodos & \multicolumn{1}{|c|}{ TNN } & \multicolumn{1}{|l}{ WNN } \\
\hline \hline AUDJPY 15 min. & 0.5242 & 0.5206 \\
\hline AUDJPY 1 hora & 0.7144 & 0.8683 \\
\hline AUDJPY 1 dia & 0.0724 & 0.1635 \\
\hline CHFJPY 15 min. & $2.03 \mathrm{e}-07$ & $1.31 \mathrm{e}-06$ \\
\hline CHFJPY 1 hora & 0.0350 & 0.0885 \\
\hline CHFJPY 1 dia & 0.8109 & 0.8714 \\
\hline EURCHF 15 min. & 1.0000 & 1.0000 \\
\hline EURCHF 1 hora & 0.6017 & 0.6017 \\
\hline EURCHF 1 dia & $9.33 \mathrm{e}-04$ & 0.2314 \\
\hline EURJPY 15 min. & 0.01728 & 0.0463 \\
\hline EURJPY 1 hora & 0.5579 & 0.5147 \\
\hline EURJPY 1 dia & 0.4749 & 0.4962 \\
\hline GBPJPY 15 min. & 0.0830 & 0.0773 \\
\hline GBPJPY 1 hora & 0.3483 & 0.5007 \\
\hline GBPJPY 1 dia & 0.4797 & 0.4674 \\
\hline
\end{tabular}

\subsection{TESTES DE CORRELAÇÃO PELO GRÁFICO DE DISPERSÃO}

Valendo-se das séries temporais de taxas de câmbio (116), apresentadas nos Testes de Normalidade, Raiz Unitária e de não-linearidade, e empregando-se novamente do ambiente de programação para análise de dados estatísticos e gráficos $R$ (47) e sua biblioteca astsa, realizou-se a execução dos Testes de Correlação servindo-se do método $\operatorname{lag}_{1}$.plot o qual fornece uma grade de gráficos de dispersão das séries em contrate com os valores defasados das séries.

A finalidade deste teste é comprovar se as defasagens das séries estudadas, $t_{-1}, t_{-2}, t_{-3}, \ldots$, $t_{-n}$, possuem correlação fraca ou mesmo não são relacionadas entre si.

A Figura 12 apresenta o gráfico de dispersão para a série temporal de relação entre as moedas Dólar Australiano (AUD) e o Yen Japonês (JPY) com periodicidade de 15 minutos.

Para os testes de correlação entre as defasagens das séries examinadas, por intermédio do método $\operatorname{lag}_{1}$.plot, somente se levou em consideração as primeiras nove defasagens, $t_{-1}, t_{-2}, t_{-3}, \ldots, t_{-9}$, visto que se constatou que mesmo nas primeiras defasagens, $t_{-1}, t_{-2} \mathrm{e}$ $t_{-3}$, a correlação entre as defasagens das séries eram muito fraca.

Foram efetuados os testes de correlação em todos os períodos das séries AUD-JPY, CHF- 

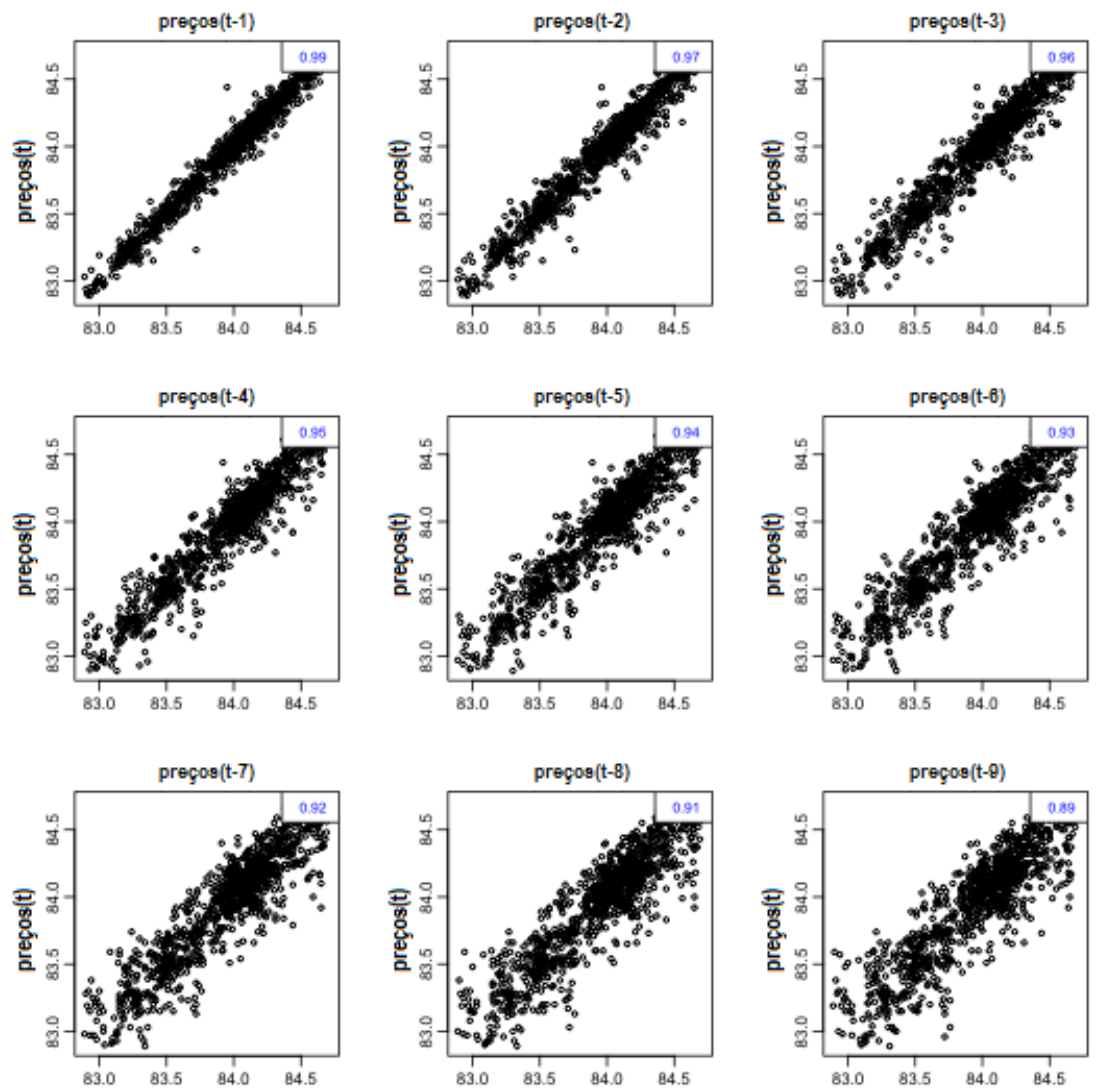

Figura 12 - Gráfico de dispersão para a relação entre as moedas AUD e JPY com periodicidade de 15 minutos.

JPY, EUR-CHF, EUR-JPY e GBP-JPY, com as periodicidades de 15 minutos, 1 hora e 1 dia. Todas elas apresentaram o mesmo padrão de correlação entre as defasagens, ou seja, possuem uma correlação fraca ou mesmo não são relacionadas entre si.

\subsection{AJUSTES POR MEIO DO MODELO SVR TRADICIONAL COM KERNEL}

O modelo SVR tradicional com Kernel mostra-se apropriado para resolver problemas de estimação não lineares de regressão, sendo uma alternativa adequada para previsão de dados não-lineares.

Mapeando-se um conjunto de treinamento de seu espaço original para um novo espaço de dimensão maior, o SVM e seu regressor SVR têm a habilidade de lidar com problemas não lineares (58) por meio do uso de funções Kernels: $K\left(x_{t}, x_{i}\right)=\Phi\left(x_{t}\right) \Phi\left(x_{i}\right)$ (60)).

Para os justes por meio do modelo SVR tradicional com Kernel optou-se pela utilização do Kernel Gaussiano (RBF): $K\left(x_{t}, x_{i}\right)=\exp \left(-\sigma\left\|x_{t} \cdot x_{i}\right\|^{2}\right)$ (131).

Para se criar um modelo SVR usando-se o ambiente de programação para análise de dados estatísticos e gráficos $R$ (47), com a sua biblioteca e1071 (90), adotou-se sobretudo dois 
métodos: svm e predict.

\subsubsection{Aplicação do Método SVR tradicional com Kernel em R}

O método svm é utilizado para treinar a Máquina de Suporte Vetorial para realizar regressões não-lineares estimando um valor condicional não esperado, enquanto que o método predict é aplicado para predizer valores futuros, previamente treinados pelo método svm. Também um terceiro método, tune, pode ser empregado para se regular os parâmetros de entrada do método svm.

A Figura 13 apresenta o gráfico da série prevista, gerada por meio dos métodos svm e pred, com seus pontos marcados com pequenos xis $(\times)$ azuis, sobreposto à série original, marcada com pequenos círculos $(\bullet)$ pretos em seus pontos, para a relação entre as moedas Dólar Australiano (AUD) e o Yen Japonês (JPY) com periodicidade de 15 minutos.

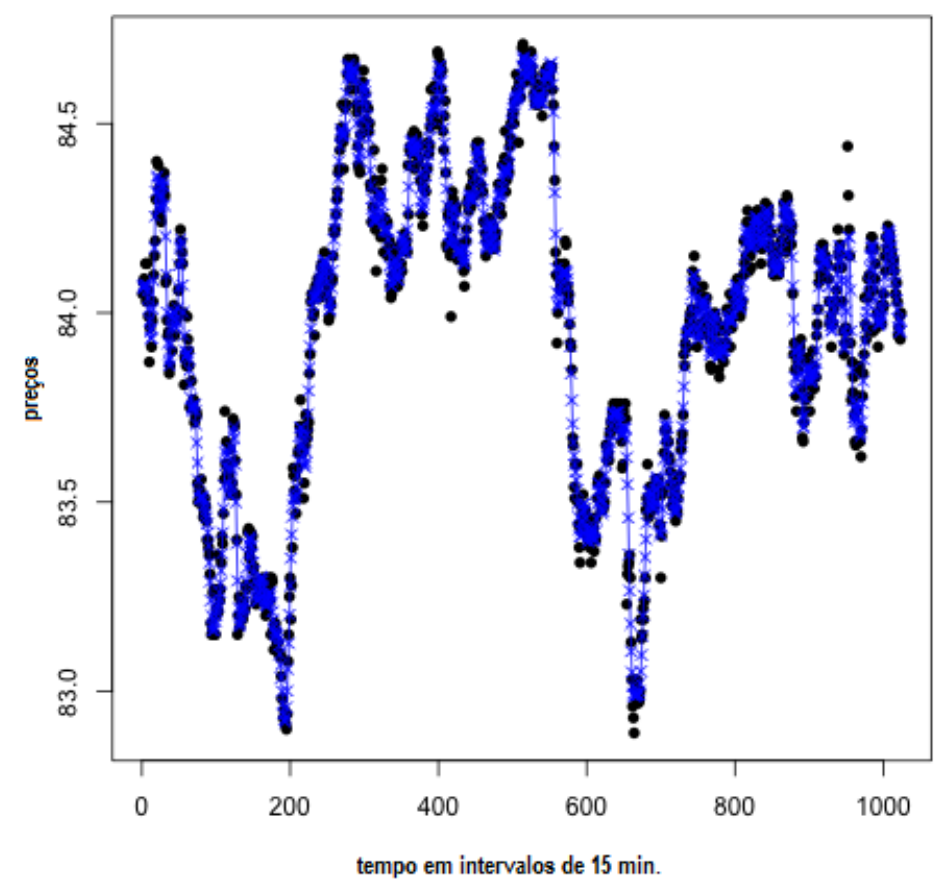

Figura 13 - Gráficos sobrepostos da série prevista $(\times)$, obtido por meio dos métodos svm e predict, e da série original (•) para a relação entre as moedas AUD e JPY com periodicidade de 15 minutos. 


\subsubsection{Qualidade da Previsão por meio do Modelo SVR tradicional com Kernel}

Analisando-se os gráficos sobrepostos, observa-se que a previsão efetuada foi adequada, que é comprovada pelas métricas adotadas.

As métricas Erro Quadrático Médio (MSE), Raiz Quadrada do Erro Quadrático Médio (RMSE), Erro Absoluto Médio (MAE), Erro Porcentual Médio (MPE), Erro Porcentual Absoluto Médio (MAPE), Erro Escalado Absoluto Médio (MASE), Autocorrelação de Erros para uma Defasagem (ACF1), Critério de Informação de Akaike (AIC), AIC de Segunda Ordem (AICc) e Critério de Informação Bayesiano (BIC) são aplicadas com o propósito de medir a qualidade do modelo (142) (94) (127).

As Tabelas 5 e 6 sintetizam os resultados encontrados, com o intuito de se aferir a qualidade do modelo apresentado por intermédio dos métodos svm e predict.

Tabela 5 - Resultados das métricas MSE, RMSE, MAE, MPE e MAPE, calculados para o modelo SVR tradicional com Kernel.

\begin{tabular}{|l|c|c|c|c|c|}
\hline Série/Métodos & MSE & RMSE & MAE & MPE & MAPE \\
\hline \hline AUDJPY 15 min. & -0.00016993720 & 0.04917307000 & 0.03522102000 & -0.00016929760 & 0.04196051000 \\
\hline AUDJPY 1 hora & 0.00317499000 & 0.16157420000 & 0.11929530000 & 0.00338283800 & 0.11881740000 \\
\hline AUDJPY 1 dia & -0.00089911060 & 0.57459460000 & 0.41281460000 & 0.00278828700 & 0.47088310000 \\
\hline CHFJPY 15 min. & -0.00438221400 & 0.10703260000 & 0.05915611000 & -0.00498914300 & 0.06676004000 \\
\hline CHFJPY 1 hora & -0.00358073200 & 0.08923372000 & 0.06850564000 & -0.00397893400 & 0.07808360000 \\
\hline CHFJPY 1 dia & 0.01369226000 & 0.76593830000 & 0.58791300000 & 0.01751008000 & 0.60531530000 \\
\hline EURCHF 15 min. & -0.00024552390 & 0.00154972200 & 0.00066504020 & -0.01579134000 & 0.04277633000 \\
\hline EURCHF 1 hora & 0.00005907420 & 0.00143734900 & 0.00071705390 & 0.00383386800 & 0.04636850000 \\
\hline EURCHF 1 dia & -0.00024107200 & 0.00767428300 & 0.00419220500 & -0.02051068000 & 0.34561970000 \\
\hline EURJPY 15 min. & 0.00138731600 & 0.06934656000 & 0.05281129000 & 0.00107364300 & 0.03951979000 \\
\hline EURJPY 1 hora & -0.00273741900 & 0.06440713000 & 0.04906748000 & -0.00195126800 & 0.03548949000 \\
\hline EURJPY 1 dia & 0.00852138300 & 0.81944750000 & 0.67583740000 & 0.01344046000 & 0.56702870000 \\
\hline GBPJPY 15 min. & 0.00101734700 & 0.04542055000 & 0.03567964000 & 0.00086960470 & 0.02994245000 \\
\hline GBPJPY 1 hora & 0.04598120000 & 0.50084810000 & 0.39570810000 & 0.02345161000 & 0.20355450000 \\
\hline GBPJPY 1 dia & 0.02547556000 & 1.00168800000 & 0.83649770000 & 0.02494798000 & 0.58638860000 \\
\hline
\end{tabular}


Tabela 6 - Resultados das métricas MASE, ACF1, AIC, AICc e BIC, calculados para o modelo SVR tradicional com Kernel.

\begin{tabular}{|l|c|c|c|c|c|}
\hline Série/Métodos & MASE & ACF1 & AIC & AICc & BIC \\
\hline \hline AUDJPY 15 min. & 1.39586200000 & 0.75221 & -3255.428 & -3255.404 & -3235.702 \\
\hline AUDJPY 1 hora & 1.72473200000 & 0.79459 & -819.089 & -819.065 & -799.363 \\
\hline AUDJPY 1 dia & 1.57735400000 & 0.83628 & 1779.209 & 1779.232 & 1798.935 \\
\hline CHFJPY 15 min. & 1.55772500000 & 0.70681 & -1662.520 & -1662.496 & -1642.794 \\
\hline CHFJPY 1 hora & 1.51748200000 & 0.76054 & -2034.998 & -2034.975 & -2015.272 \\
\hline CHFJPY 1 dia & 2.06830800000 & 0.84266 & 2367.879 & 2367.903 & 2387.605 \\
\hline EURCHF 15 min. & 2.61667800000 & 0.46447 & -10335.920 & -10335.890 & -10316.190 \\
\hline EURCHF 1 hora & 2.82133100000 & 0.39520 & -10490.080 & -10490.060 & -10470.360 \\
\hline EURCHF 1 dia & 1.43546200000 & 0.65876 & -7059.529 & -7059.506 & -7039.803 \\
\hline EURJPY 15 min. & 1.67535200000 & 0.82285 & -2551.386 & -2551.363 & -2531.660 \\
\hline EURJPY 1 hora & 1.64381700000 & 0.78528 & -2702.717 & -2702.694 & -2682.991 \\
\hline EURJPY 1 dia & 2.02889900000 & 0.85889 & 2506.178 & 2506.202 & 2525.904 \\
\hline GBPJPY 15 min. & 1.71375800000 & 0.77196 & -3418.001 & -3417.978 & -3398.275 \\
\hline GBPJPY 1 hora & 1.87251300000 & 0.76520 & 1497.892 & 1497.915 & 1517.617 \\
\hline GBPJPY 1 dia & 2.33357300000 & 0.89099 & 2917.440 & 2917.463 & 2937.166 \\
\hline
\end{tabular}

\subsection{AJUSTES POR WAVELET: TRANSFORMADA E INVERSA}

Para se decompor uma série em coeficientes wavelet mediante o ambiente de programação para análise de dados estatísticos e gráficos $R$ (47), com a sua biblioteca wavethresh (98), e posterior recomposição das séries originais, este trabalho se valeu de dois métodos: $w d$ e $w r$.

\subsubsection{Aplicação da Transformada wavelet e sua Inversa em R}

O método $w d$ é utilizado para efetuar a transformada wavelet, enquanto que o método $w r$ é aplicado para executar a transformada wavelet Inversa.

Somente com o propósito de ilustração das qualidades de representação da transformada wavelet, a Figura 14 expõe o gráfico da série original com seus pontos marcados com pequenos círculos $(\bigcirc)$ azuis, e sua Transformada wavelet inversa, marcada com pequenos xis $(\times)$ vermelhos em seus pontos, gerada por meio dos métodos $w d$ e $w r$, sobreposta à série original para a relação entre as moedas Dólar Australiano (AUD) e o Yen Japonês (JPY) com periodicidade de 15 minutos. 


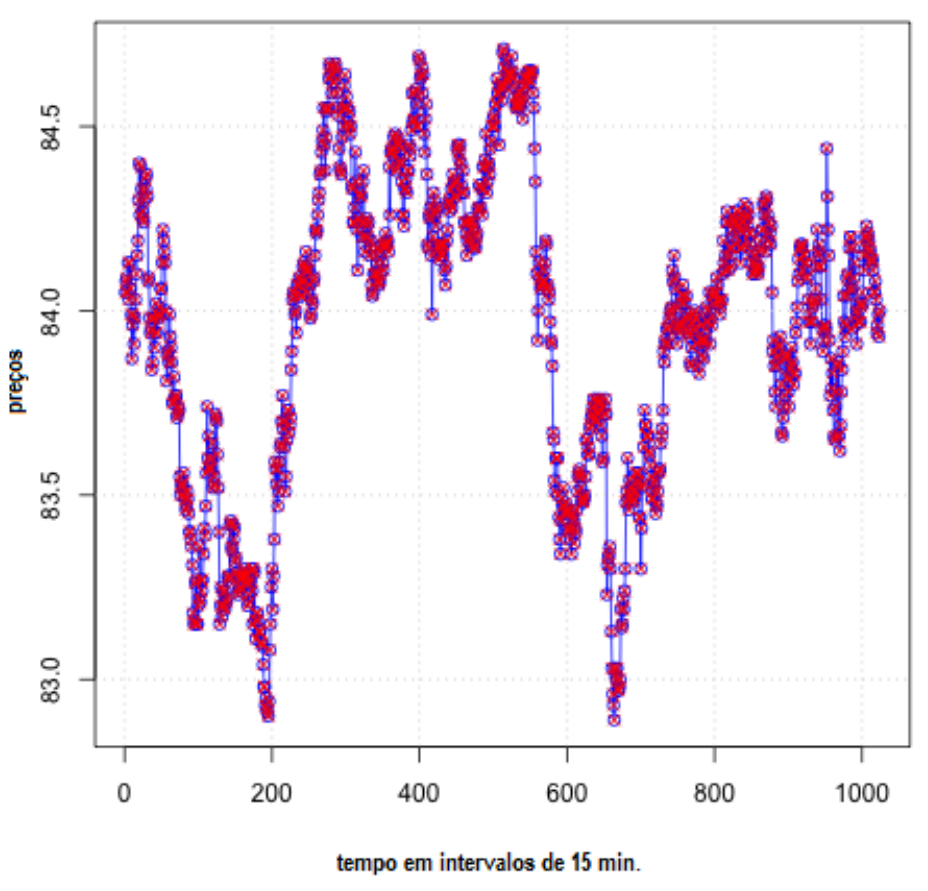

Figura 14 - Gráficos sobrepostos da série original $(\bigcirc)$ e sua Transformada wavelet inversa $(\times)$ para a relação entre as moedas AUD e JPY com periodicidade de 15 minutos.

\subsubsection{Qualidade da Transformada wavelet e sua Inversa}

Analisando-se os gráficos sobrepostos, observa-se que a Transformada wavelet inversa é muito semelhante, senão idêntica à série original. Por certo esta característica ou mesmo esta similitude entre os gráficos já era esperada, senão toda formulação do modelo híbrido SVR-wavelet, valendo-se de wavelets, não teria sentido.

Assim, somente a título de dilucidação, efetuando-se algumas métricas entre os pontos das séries originais e suas Transformadas wavelet inversas chega-se a medidas muito pequenas, como por exemplo a $R M S E=3.449136 e-09$, o que já era previsível.

\subsection{AJUSTES POR WAVELET MODWT UTILIZANDO A WAVELET HAAR}

Outra possibilidade para se decompor uma série em coeficientes wavelet, através da aplicação do ambiente de programação para análise de dados estatísticos e gráficos $R$ (47), com a sua biblioteca wmtsa e posterior recomposição da série original, é pela utilização dos métodos wavMODWT e reconstruct. 


\subsubsection{Aplicação da wavelet MODWT e sua Inversa em R}

O método wavMODWT é usado para efetuar a Transformada wavelet Discreta de Máxima Sobreposição (MODWT), à medida que o método reconstruct é utilizado para realizar o cálculo de sua Transformada inversa, escopo do estudo deste trabalho.

Novamente, meramente com o intuito de elucidação das propriedades de representação da transformada wavelet MODWT, a Figura 15 exibe o gráfico da série original com seus pontos destacados com pequenos triângulos $(\triangle)$ azuis, e sua Transformada wavelet MODWT inversa, destacados com pequenos losangos $(\diamond)$ vermelhos em seus pontos, obtida por meio dos métodos wavMODWT e reconstruc, sobreposta à série original para a relação entre as moedas Dólar Australiano (AUD) e o Yen Japonês (JPY) com periodicidade de 15 minutos.

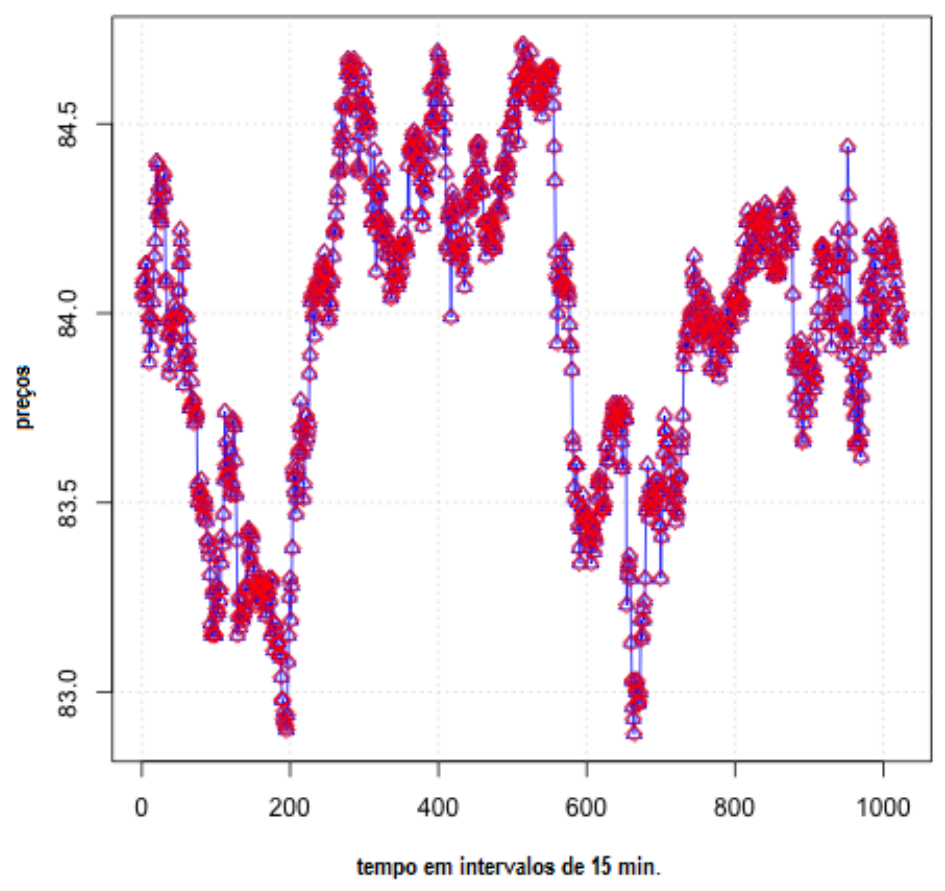

Figura 15 - Gráficos sobrepostos da série original $(\triangle)$ e sua Transformada wavelet MODWT inversa $(\diamond)$ para a relação entre as moedas AUD e JPY com periodicidade de 15 minutos.

\subsubsection{Qualidade da wavelet MODWT e sua Inversa}

Averiguando-se os gráficos justapostos, constata-se que a Transformada wavelet MODWT inversa é análoga, senão equivalente à série original. Como previamente clarificado na seção que aludiu à Transformada wavelet e sua Inversa, esta identidade ou mesmo esta similaridade 
entre os gráficos já era esperada, caso contrário toda concepção e elaboração do modelo híbrido SVR-wavelet, beneficiando-se das wavelets, não teria acepção.

Ademais, unicamente a título de esclarecimento, realizando-se algumas medidas entre os pontos das séries originais e suas Transformadas wavelet MODWT inversas chega-se à dimensões muito pequenas. Deveras, a $R M S E=1.424281 e-13$, o que já era aguardada.

\subsection{A DECOMPOSIÇÃO EM WAVELETS}

O princípio da análise wavelet é a de expressar ou aproximar um sinal ou função por uma família de funções geradas por dilatações e translações de um wavelet mãe.

Depois da execução dos testes com o método wavMODWT obteve-se novas séries como resultado da decomposição dos componentes de aproximação e detalhes, respectivamente $s 5$ e $d 1$ a $d 5$.

Pode-se comprovar a decomposição produzida pela somatória dos valores de cada componente, que deve ser igual ao valor original da série.

Tomando-se como exemplo o primeiro componente de cada detalhe, $d 1$ a $d 5$, e o correspondente componente de aproximação, s5, com a aplicação de técnicas de denoising ou wavelet shrinkage para eliminação de ruídos contidos na série, todos com índice [1], e o primeiro valor da série original, constata-se sua soma e igualdade, conforme:

- prices.modwt $\$ d a t a \$ d 1[1]=0.0112905004969619$,

- prices.modwt $\$ d a t a \$ d 2[1]=0.00883984638146273$,

- prices.modwt\$data $\$ d 3[1]=-0.0129682480685034$,

- prices.modwt\$data $\$ d 4[1]=-0.0605947777243614$,

- prices.modwt $\$ d a t a \$ d 5[1]=-0.00706702507418555$,

- prices.modwt $\$ d a t a \$ s 5[1]=84.0763590584155 \mathrm{e}$

- prices $2 . t s[1]=84.015859$

onde prices.modwt é a variável de saída do método wavMODWT, data são os componentes obtidos, contento os valores de aproximação e detalhes, respectivamente $s 5$ e $d 1$ a $d 5$, e prices2.ts é o vetor contendo a série original, tal que:

$$
\begin{aligned}
& {[(0.0112905004969619)+(0.00883984638146273)+(-0.0129682480685034)+} \\
& (-0.0605947777243614)+(-0.00706702507418555)+(84.0763590584155)]=84.015859 .
\end{aligned}
$$


A decomposição dos componentes de aproximação e detalhes por intermédio da Transformada wavelet MODWT, para a relação entre as moedas Dólar Australiano (AUD) e o Yen Japonês (JPY) com periodicidade de 15 minutos, é exposta na Figura 16, em que também é apresentada, no topo da Figura, a série original.

\section{MODWT - usando wavelet Haar}

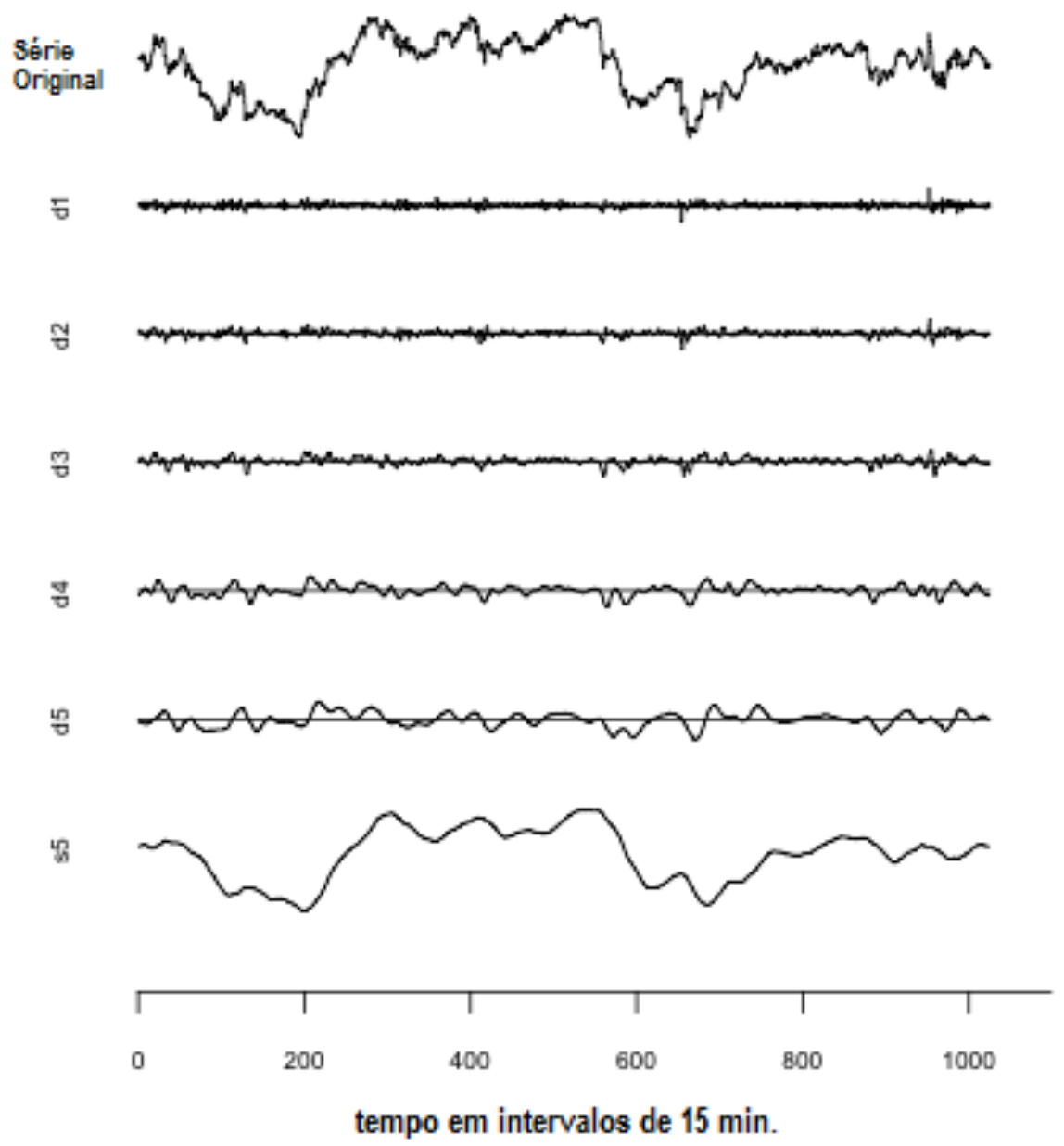

Figura 16 - Componentes de aproximação e detalhes, respectivamente $s 5$ e $d 1$ a $d 5$, decompostos pela MODWT, para a relação entre as moedas AUD e JPY com periodicidade de 15 minutos.

Percebe-se, pelos pontos das séries obtidas como resultado da decomposição dos componentes de aproximação, $s 5$, e os detalhes, $d 1$ a $d 5$, que a distribuição de energia está concentrada no componente de aproximação, s5, em concordância com que é exibido na Figura 17, pelos gráficos de Distribuição de Energia e seu equivalente Box Plot. 
Distribuição de Energia

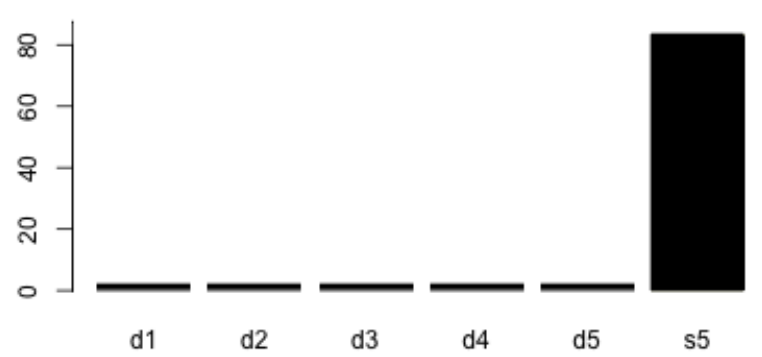

Estatisticas de Distribuição

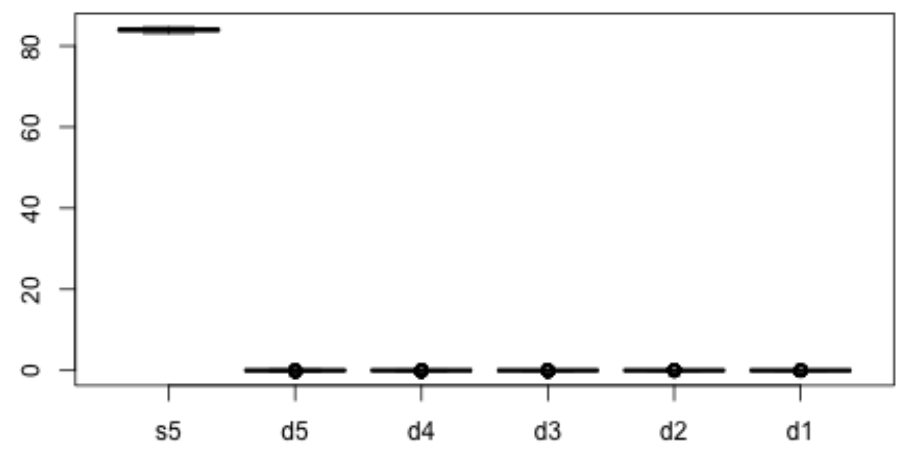

Figura 17 - Gráfico de Distribuição de Energia e seu equivalente Box Plot dos componentes de aproximação e detalhes, respectivamente $s 5$ e $d 1$ a $d 5$, decompostos pela MODWT, para a relação entre as moedas AUD e JPY com periodicidade de 15 minutos.

\subsection{MODELO HÍBRIDO SVR-WAVELET - AJUSTES E PREVISÃO DOS COMPONENTES WAVELETS}

As wavelets possibilitam a eliminação de ruídos por meio de técnicas de denoising ou wavelet shrinkage. Por intermédio da transformada wavelet, uma parte do ruído é removida da série original antes da extração de atributos (129). A técnica de wavelet denoising tenta transformar $y(n)$ novamente em $x(n)$, ou pelo menos em um sinal similar, como pode ser percebido na Figura 18, que ilustra a decomposição da série para a relação entre as moedas AUD e JPY com periodicidade de 15 minutos, através da Transformada wavelet MODWT, com suas componentes de aproximação e detalhes, respectivamente $s 5$ e $d 1$ a $d 5$, em comparação com a aplicação de técnicas de denoising ou wavelet shrinkage para eliminação de ruídos contidos na série.

Pelos gráficos apresentados na Figura 18, identifica-se que a série original supostamente possui adição de um ruído $r(n)$, assim como ela também revela outro componente, o de tendência $T(n)$, comumente encontrado nas séries FOREX (116), estudo deste trabalho.

Também pela apresentação e comparação entre a série original e os componentes de apro- 
ximação, $s 5$, e detalhes, $d 1$ a $d 5$, sobrevém que as componentes são suavizações da série original, proporcionada pela Transformada wavelet MODWT, principalmente após a aplicação de técnicas de denoising ou wavelet shrinkage para eliminação de ruídos contidos na série.

A decomposição dos componentes de aproximação e detalhes são utilizados como variáveis de entrada do SVR para prever dados futuros dos ativos financeiros.

Dessa maneira a Transformada wavelet MODWT é colocada como um pré-processador do conjuntos de dados de entrada, conforme ilustrado pela Figura 8 modelo proposto para predição de séries temporais por meio da estratégia SVR-wavelet híbrida.

\subsubsection{Qualidade da Previsão por meio do Modelo Híbrido SVR-wavelet}

As métricas MSE, RMSE, MAE, MPE, MAPE, MASE, ACF1, AIC, AICc e BIC também são empregadas com a finalidade de medir a qualidade do modelo híbrido proposto.

São exibidos os resultados das métricas MSE, RMSE, MAE, MPE, MAPE, MASE, ACF1, $\mathrm{AIC}, \mathrm{AICc}$ e BIC, segundo suas respectivas definições, anteriormente aludidas neste trabalho. As Tabelas 7 e 8 condensam os resultados obtidos, com o propósito de verificar a qualidade do modelo híbrido proposto, como um pré-processador do conjuntos de dados de entrada, com o objetivo de prever dados futuros dos ativos financeiros do tipo FOREX, em concordância com a Figura 8 .

\subsection{AJUSTES POR MEIO DO MODELO ARIMA}

Um modelo tradicional frequentemente utilizado como regressor de séries temporais financeiras é o modelo Autorregressivo Integrado de Médias Móveis (ARIMA) de ordem $(p, d, q)$.

As séries ajustadas por intermédio do modelo híbrido SVR-wavelet podem ser comparadas com este modelo.

Em $R$, servindo-se da biblioteca astsa (127), é possível ajustar as séries originais ao modelo ARIMA mediante à aplicação do método sarima, bem como realizar previsões $n$ passos à frente de séries temporais através de seu método sarima.for.

\subsubsection{Qualidade da Previsão por meio do Modelo ARIMA}

Identicamente, as métricas MSE, RMSE, MAE, MPE, MAPE, MASE, ACF1, AIC, AICc e BIC são lidadas para comparar as características do modelo ARIMA em oposição ao modelo proposto baseado em um sistema SVR-wavelet híbrido.

São denotados os produtos das métricas MSE, RMSE, MAE, MPE, MAPE, MASE, ACF1, 
Tabela 7 - Resultados das métricas MSE, RMSE, MAE, MPE e MAPE, calculados para o modelo híbrido SVR-wavelet.

\begin{tabular}{|l|c|c|c|c|c|}
\hline Série/Métodos & MSE & RMSE & MAE & MPE & MAPE \\
\hline \hline AUDJPY 15 min. & 0.003769474 & 0.029037880 & 0.021846580 & 0.004395223 & 0.026029350 \\
\hline AUDJPY 1 hora & -0.008094633 & 0.123794100 & 0.101444300 & -0.009831646 & 0.101238000 \\
\hline AUDJPY 1 dia & -0.000946800 & 0.392750200 & 0.340413300 & -0.027584370 & 0.387377100 \\
\hline CHFJPY 15 min. & 0.002622926 & 0.065786500 & 0.043294690 & 0.002616942 & 0.048863600 \\
\hline CHFJPY 1 hora & 0.006250253 & 0.073482910 & 0.057785630 & 0.006381544 & 0.065816800 \\
\hline CHFJPY 1 dia & -0.035338230 & 0.709598120 & 0.619334730 & -0.109489280 & 0.638716170 \\
\hline EURCHF 15 min. & -0.000065229 & 0.000983200 & 0.000404835 & -0.004274114 & 0.026048590 \\
\hline EURCHF 1 hora & 0.007886270 & 0.046991400 & 0.040134880 & 0.005567794 & 0.029022230 \\
\hline EURCHF 1 dia & -0.034075460 & 0.815840230 & 0.744326040 & -0.102338860 & 0.628002860 \\
\hline EURJPY 15 min. & -0.000433032 & 0.047534990 & 0.041100150 & -0.000509092 & 0.030760350 \\
\hline EURJPY 1 hora & 0.007886270 & 0.046991400 & 0.040134880 & 0.005567794 & 0.029022230 \\
\hline EURJPY 1 dia & -0.034075460 & 0.815840230 & 0.744326040 & -0.102338860 & 0.628002860 \\
\hline GBPJPY 15 min. & 0.005416988 & 0.041690770 & 0.033354460 & 0.004417095 & 0.027985000 \\
\hline GBPJPY 1 hora & -0.046711990 & 0.500528910 & 0.377088290 & -0.030638720 & 0.192854460 \\
\hline GBPJPY 1 dia & -0.114355100 & 1.104917200 & 0.975509100 & -0.164341200 & 0.685634500 \\
\hline
\end{tabular}

AIC, AICc e BIC, segundo suas concernentes temáticas, supradito neste relatório. As Tabelas 9 e 10 compendiam os resultados apurados, com o propósito de validar a qualidade do modelo ARIMA, apresentado por meio dos métodos sarima e sarima.for, para previsão de dados de ativos FOREX e conseguinte comparação com o modelo proposto baseado em um sistema SVR-wavelet híbrido, evidenciado anteriormente pela Figura 8 .

\subsection{AJUSTES POR MEIO DO MODELO ARFIMA}

As séries ajustadas por intermédio do modelo híbrido SVR-wavelet podem ser comparadas com outros modelos tradicionais tais como o modelo Autorregressivo Fracionário Integrado de Médias Móveis (ARFIMA) ou ARIMA Fracionário.

Valendo-se do ambiente de programação para análise de dados estatísticos e gráficos $R$ (47), provido da biblioteca forecast (63), é possível ajustar as séries originais mediante à aplicação do método arfima bem como realizar previsões $n$ passos à frente de séries temporais ou modelos de séries temporais, através de seu método forecast. 
Tabela 8 - Resultados das métricas MASE, ACF1, AIC, AICc e BIC, calculados para o modelo híbrido SVRwavelet.

\begin{tabular}{|l|c|c|c|c|c|}
\hline Série/Métodos & MASE & ACF1 & AIC & AICc & BIC \\
\hline \hline AUDJPY 15 min. & 0.865812800 & 0.479377200 & -4334.202 & -4334.178 & -4314.476 \\
\hline AUDJPY 1 hora & 1.466648000 & 0.709609300 & -1364.563 & -1364.540 & -1344.837 \\
\hline AUDJPY 1 dia & 1.300711000 & 0.754438200 & 999.963 & 999.987 & 1019.689 \\
\hline CHFJPY 15 min. & 1.140055000 & 0.390209200 & -2659.319 & -2659.296 & -2639.594 \\
\hline CHFJPY 1 hora & 1.280021000 & 0.646169300 & -2432.732 & -2432.709 & -2413.006 \\
\hline CHFJPY 1 dia & 2.178850780 & 0.805693210 & 2211.406 & 2211.430 & 2231.132 \\
\hline EURCHF 15 min. & 1.592869000 & -0.207162200 & -11267.800 & -11267.770 & -11248.070 \\
\hline EURCHF 1 hora & 1.344564000 & 0.693024700 & -3348.369 & -3348.345 & -3328.643 \\
\hline EURCHF 1 dia & 2.234505490 & 0.859891060 & 2497.143 & 2497.166 & 2516.869 \\
\hline EURJPY 15 min. & 1.303835000 & 0.758676700 & -3324.814 & -3324.791 & -3305.088 \\
\hline EURJPY 1 hora & 1.344564000 & 0.693024700 & -3348.369 & -3348.345 & -3328.643 \\
\hline EURJPY 1 dia & 2.234505490 & 0.859891060 & 2497.143 & 2497.166 & 2516.869 \\
\hline GBPJPY 15 min. & 1.602075000 & 0.645801500 & -3593.484 & -3593.460 & -3573.758 \\
\hline GBPJPY 1 hora & 1.784403220 & 0.661645370 & 1496.586 & 1496.609 & 1516.312 \\
\hline GBPJPY 1 dia & 2.721371700 & 0.849545000 & 3118.316 & 3118.339 & 3138.042 \\
\hline
\end{tabular}

\subsubsection{Qualidade da Previsão por meio do Modelo ARFIMA}

Similarmente, as métricas MSE, RMSE, MAE, MPE, MAPE, MASE, ACF1, AIC, AICc e BIC são aplicadas com a função de aferir as características do modelo ARFIMA.

São expostos os resultados das métricas MSE, RMSE, MAE, MPE, MAPE, MASE, ACF1, AIC, AICc e BIC, segundo seus respectivos conteúdos, antecedentemente referidos neste relatório. As Tabelas 11 e 12 condensam os resultados constatados, com o propósito de validar a qualidade do modelo ARFIMA, apresentado por meio dos métodos arfima e forecast, para previsão de dados de ativos FOREX e consequente comparação com o modelo proposto baseado em um sistema SVR-wavelet híbrido.

\subsection{AJUSTES POR MEIO DO MODELO GARCH}

O modelo GARCH, Autorregressivo Condicional com Heterocedasticidade Generalizado, é outro modelo tradicionalmente usado como regressor de séries temporais financeiras.

As séries ajustadas por intermédio do modelo híbrido SVR-wavelet podem ser contrastadas 
Tabela 9 - Resultados das métricas MSE, RMSE, MAE, MPE e MAPE, calculados para o modelo ARIMA.

\begin{tabular}{|l|c|c|c|c|c|}
\hline Série/Métodos & MSE & RMSE & MAE & MPE & MAPE \\
\hline \hline AUDJPY 15 min. & 0.0000628946 & 0.06768088 & 0.04866654 & 0.0000400302 & 0.05797705 \\
\hline AUDJPY 1 hora & -0.0000317264 & 0.18769970 & 0.13045890 & -0.0001293830 & 0.12989150 \\
\hline AUDJPY 1 dia & -0.0001910640 & 0.69370640 & 0.51200940 & -0.0036240220 & 0.58391940 \\
\hline CHFJPY 15 min. & 0.0000811184 & 0.10899270 & 0.06286427 & 0.0000026521 & 0.07099687 \\
\hline CHFJPY 1 hora & 0.0000539385 & 0.11706850 & 0.08491374 & 0.0000040283 & 0.09680131 \\
\hline CHFJPY 1 dia & 0.0001968890 & 0.72047260 & 0.49741820 & -0.0040388630 & 0.51354630 \\
\hline EURCHF 15 min. & 0.0000015143 & 0.00139596 & 0.00032705 & 0.0000468887 & 0.02103233 \\
\hline EURCHF 1 hora & 0.0000015047 & 0.00147483 & 0.00031272 & 0.0000330088 & 0.02020792 \\
\hline EURCHF 1 dia & 0.0000003992 & 0.00700141 & 0.00326238 & -0.0018331130 & 0.26838970 \\
\hline EURJPY 15 min. & 0.000140960 & 0.08862456 & 0.06608863 & 0.0000844043 & 0.04947692 \\
\hline EURJPY 1 hora & 0.000135040 & 0.07649206 & 0.05382387 & 0.0000776646 & 0.03891701 \\
\hline EURJPY 1 dia & -0.004769378 & 0.87363660 & 0.63969740 & -0.0086722430 & 0.54034920 \\
\hline GBPJPY 15 min. & 0.000114738 & 0.05766623 & 0.04159196 & 0.0000809079 & 0.03489244 \\
\hline GBPJPY 1 hora & 0.000167809 & 0.51821260 & 0.34059260 & -0.0002383680 & 0.17637870 \\
\hline GBPJPY 1 dia & 0.015015380 & 0.93259160 & 0.68259830 & 0.0049445250 & 0.47596490 \\
\hline
\end{tabular}

com o modelo ARCH Generalizado de ordem $(m, s)$ ou simplesmente $\operatorname{GARCH}(m, s)$.

No ambiente de programação para análise de dados estatísticos e gráficos $R$ (47), e suas bibliotecas, em particular a biblioteca fGarch (142), é possível ajustar as séries originais mediante à aplicação do método garchFit, bem como realizar previsões $n$ passos à frente de séries temporais através do método predict.

\subsubsection{Qualidade da Previsão por meio do Modelo GARCH}

A obtenção dos valores gerados pelo modelo GARCH não são triviais em $R$. Seus métodos somente expõem dados de características estatísticas do modelo, encapsulando os demais valores. Entretanto algumas métricas, como os valores AIC e BIC, são expostos e são utilizados para se comparar suas características àquelas do modelo proposto baseado em um sistema SVR-wavelet híbrido.

Portanto, para esta comparação, somente são empregados os valores das métricas AIC e BIC. A Tabela 13 sintetiza os resultados obtidos, com o propósito de validar a qualidade do modelo GARCH, apresentado por meio dos métodos garchFit e predict, para previsão de dados de ativos FOREX e subsequente comparação com o modelo proposto baseado em um 
Tabela 10 - Resultados das métricas MASE, ACF1, AIC, AICc e BIC, calculados para o modelo ARIMA.

\begin{tabular}{|l|c|c|c|c|c|}
\hline Série/Métodos & MASE & ACF1 & AIC & AICc & BIC \\
\hline \hline AUDJPY 15 min. & 0.9979129 & 0.004630544 & -2601.179 & -2601.155 & -2581.453 \\
\hline AUDJPY 1 hora & 1.0012720 & -0.007479716 & -512.137 & -512.113 & -492.411 \\
\hline AUDJPY 1 dia & 0.9895630 & 0.000153460 & 2165.019 & 2165.043 & 2184.745 \\
\hline CHFJPY 15 min. & 0.9982948 & 0.010324950 & -1625.353 & -1625.330 & -1605.627 \\
\hline CHFJPY 1 hora & 1.0003080 & -0.000330550 & -1478.966 & -1478.942 & -1459.240 \\
\hline CHFJPY 1 dia & 0.9945253 & 0.011852200 & 2242.554 & 2242.577 & 2262.280 \\
\hline EURCHF 15 min. & 1.2868190 & -0.018625390 & -10549.910 & -10549.890 & -10530.190 \\
\hline EURCHF 1 hora & 1.2304390 & 0.000390112 & -10437.360 & -10437.340 & -10417.630 \\
\hline EURCHF 1 dia & 1.0871060 & -0.007935175 & -7247.461 & -7247.438 & -7227.736 \\
\hline EURJPY 15 min. & 0.9986509 & -0.008755548 & -2049.027 & -2049.003 & -2029.301 \\
\hline EURJPY 1 hora & 1.0036790 & 0.030916450 & -2350.538 & -2350.514 & -2330.812 \\
\hline EURJPY 1 dia & 0.9977746 & -0.000628297 & 2637.320 & 2637.344 & 2657.046 \\
\hline GBPJPY 15 min. & 1.0020860 & -0.015329730 & -2929.129 & -2929.105 & -2909.403 \\
\hline GBPJPY 1 hora & 1.0003050 & -0.002158059 & 1567.693 & 1567.717 & 1587.419 \\
\hline GBPJPY 1 dia & 0.9906483 & 0.030577950 & 2771.061 & 2771.084 & 2790.786 \\
\hline
\end{tabular}

sistema SVR-wavelet híbrido, mostrado previamente pela Figura 8

\subsection{PARTE EXPERIMENTAL DO EXPOENTE DE HURST}

Para evidenciar que as séries preditas obtidas após o ajuste por intermédio do modelo híbrido proposto, com o objetivo de prever dados futuros dos ativos financeiros do tipo FOREX, em concordância com a Figura 8 , são processos Hurst ou passeios aleatórios enviesados, procedeuse a execução das tarefas conforme método adotado, preliminarmente singularizado por este trabalho.

Para obtenção da média $\left(E_{m}\right)$ e do desvio padrão $\left(S_{m}\right)$ e geração das subséries $\left(Z_{i, m}\right)$ foram utilizadas três funções, desenvolvidas e escritas no ambiente de programação para análise de dados estatísticos e gráficos $R$ (47), conforme fontes de programação anexos a este trabalho.

Para normalização dos dados da subsérie $\left(Z_{i, m}\right)$, bem como a geração da série de tempo acumulativa $(Y i, m)$, foi utilizada a função cumsum (Cumulative Sums), disponível nas bibliotecas padrões do ambiente para análise de dados estatísticos $R_{\odot}$ (47); fontes de programação estão disponíveis no anexo a este trabalho.

O restante das tarefas descritas no método, para o cálculo $R_{m}$ e da média do $R_{m} / S_{m}$, foram 
Tabela 11 - Resultados das métricas MSE, RMSE, MAE, MPE e MAPE, calculados para o modelo ARFIMA.

\begin{tabular}{|l|c|c|c|c|c|}
\hline Série/Métodos & MSE & RMSE & MAE & MPE & MAPE \\
\hline \hline AUDJPY 15 min. & -0.000038411 & 0.06753559 & 0.0486958 & -0.000112369 & 0.0580122 \\
\hline AUDJPY 1 hora & 0.006240266 & 0.22181590 & 0.1499488 & 0.005092418 & 0.1493700 \\
\hline AUDJPY 1 dia & 0.041078600 & 0.74568730 & 0.5494820 & 0.027758120 & 0.6249453 \\
\hline CHFJPY 15 min. & 0.000519787 & 0.10898450 & 0.0628009 & 0.000427509 & 0.0709306 \\
\hline CHFJPY 1 hora & -0.003390516 & 0.14242530 & 0.0961857 & -0.004420334 & 0.1095720 \\
\hline CHFJPY 1 dia & 0.073625970 & 0.85052643 & 0.5848464 & 0.042137390 & 0.6038383 \\
\hline EURCHF 15 min. & -0.000001015 & 0.00137549 & 0.0004302 & -0.000144365 & 0.0276688 \\
\hline EURCHF 1 hora & -0.000001386 & 0.00147177 & 0.0003899 & -0.000180425 & 0.0251959 \\
\hline EURCHF 1 dia & -0.000088755 & 0.00707360 & 0.0032534 & -0.010404500 & 0.2675972 \\
\hline EURJPY 15 min. & -0.004310275 & 0.09684945 & 0.0708941 & -0.003351474 & 0.0530655 \\
\hline EURJPY 1 hora & -0.005789479 & 0.08151225 & 0.0586681 & -0.004299801 & 0.0424261 \\
\hline EURJPY 1 dia & 0.092424080 & 0.99269800 & 0.7342927 & 0.042058350 & 0.6181907 \\
\hline GBPJPY 15 min. & -0.005443010 & 0.06888378 & 0.0470746 & -0.004665444 & 0.0394940 \\
\hline GBPJPY 1 hora & -0.044079550 & 0.68078874 & 0.4220548 & -0.026015220 & 0.2174034 \\
\hline GBPJPY 1 dia & 0.130793820 & 1.09033803 & 0.8086128 & 0.055316480 & 0.5615499 \\
\hline
\end{tabular}

obtidos por meio de operações e funções matemáticas convencionais; fontes de programação também disponíveis no anexo a este trabalho.

A Tabela 14 sumariza parcialmente os valores de $t, M, R / S_{t}, x=\log \left\{R / S_{t}\right\}$ e $y=\log \{t\}$, para relação predita entre as moedas AUD-JPY, com periodicidade de 1 dia, onde log é o logaritmo natural de um número, e $(x, y)$ são os pontos do gráfico com dispersão dos valores de $\log \left\{R / S_{t}\right\}$ mostrados na Figura 19 .

A Figura 19 mostra o gráfico com dispersão dos valores de $\log \left\{R / S_{t}\right\}$ em relação aos valores de $\log \{t\}$, para a relação entre as moedas $A U D-J P Y$, com periodicidade de 1 dia. A Figura 19 também apresenta a reta de tendência obtida por meio da função $l m$ (Fitting Linear Models) e abline (Add Straight Lines to a Plot), disponíveis nas bibliotecas padrões do ambiente para análise de dados estatísticos $R$ (47).

A Tabela 15 apresenta os valores para a estimativa do intercepto $\hat{b}$ e a inclinação da reta do gráfico contido na Figura 19. Esta inclinação representa o valor estimado do expoente de Hust $(\hat{H})$. Estes valores foram obtidos por meio da função $l m$ (Fitting Linear Models), summary (Summary of the Results of Model Fitting Function) e coef (Extracts Model Coefficients from Modeling Function), também disponíveis nas bibliotecas padrões do ambiente para análise de dados estatísticos $R$ (47). 
Tabela 12 - Resultados das métricas MASE, ACF1, AIC, AICc e BIC, calculados para o modelo ARFIMA.

\begin{tabular}{|l|c|c|c|c|c|}
\hline Série/Métodos & MASE & ACF1 & AIC & AICc & BIC \\
\hline \hline AUDJPY 15 min. & 0.998513 & 0.00141561 & -2605.580 & -2605.556 & -2585.854 \\
\hline AUDJPY 1 hora & 1.150856 & -0.09768307 & -170.112 & -170.089 & -150.387 \\
\hline AUDJPY 1 dia & 1.061987 & -0.00038125 & 2313.003 & 2313.026 & 2332.729 \\
\hline CHFJPY 15 min. & 0.997288 & -0.04490894 & -1625.507 & -1625.483 & -1605.781 \\
\hline CHFJPY 1 hora & 1.133095 & -0.10913820 & -1077.437 & -1077.414 & -1057.712 \\
\hline CHFJPY 1 dia & 1.169327 & -0.07730520 & 2582.415 & 2582.439 & 2602.141 \\
\hline EURCHF 15 min. & 1.692863 & 0.00310802 & -10580.170 & -10580.150 & -10560.450 \\
\hline EURCHF 1 hora & 1.534088 & 0.00097054 & -10441.610 & -10441.590 & -10421.880 \\
\hline EURCHF 1 dia & 1.084101 & 0.01852829 & -7226.452 & -7226.428 & -7206.726 \\
\hline EURJPY 15 min. & 1.071266 & -0.02741805 & -1867.270 & -1867.246 & -1847.544 \\
\hline EURJPY 1 hora & 1.094012 & 0.02137883 & -2220.354 & -2220.330 & -2200.628 \\
\hline EURJPY 1 dia & 1.145321 & 0.00634013 & 2898.977 & 2899.000 & 2918.703 \\
\hline GBPJPY 15 min. & 1.134180 & -0.08975571 & -2565.099 & -2565.076 & -2545.373 \\
\hline GBPJPY 1 hora & 1.239556 & -0.17945516 & 2126.523 & 2126.547 & 2146.249 \\
\hline GBPJPY 1 dia & 1.173532 & -0.01514771 & 3091.113 & 3091.137 & 3110.839 \\
\hline
\end{tabular}



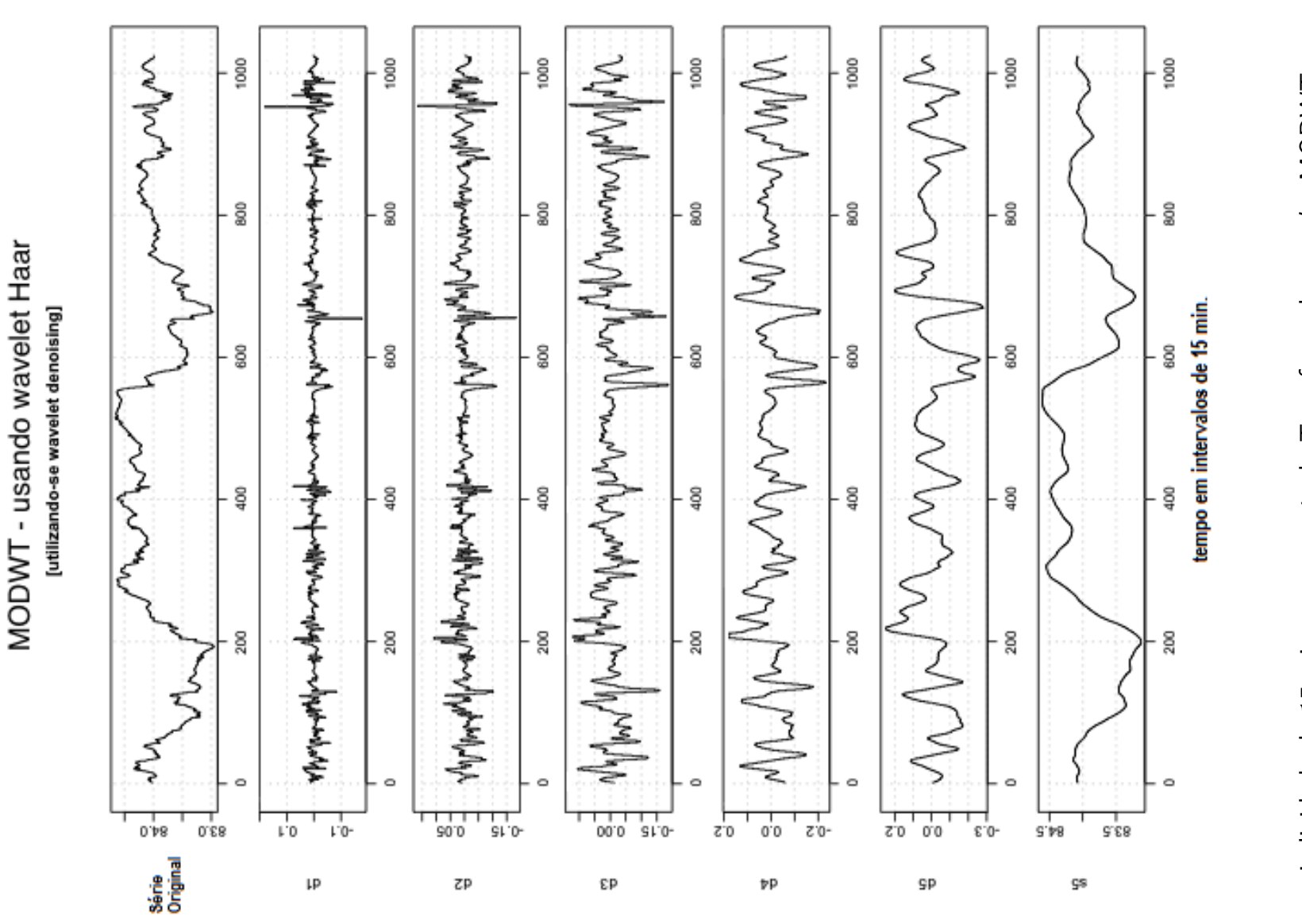

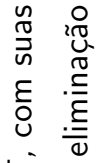

$5 \frac{\pi}{\pi}$

일

$\stackrel{\overrightarrow{\mathrm{g}}}{\mathrm{g}}$

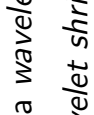

旅

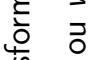

क.

중

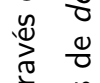

亲

告

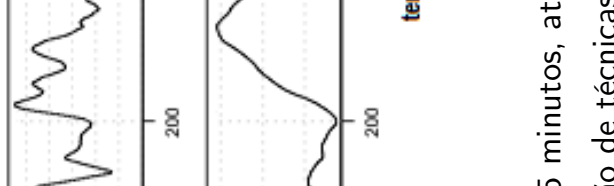

뇩

$\div$

음

:-0

产

ह

¿

० E

용

坔

है 
Tabela 13 - Resultados das métricas AIC e BIC, calculados pelo modelo GARCH.

\begin{tabular}{|l|c|c|}
\hline Série/Métodos & \multicolumn{1}{|c|}{ AIC } & \multicolumn{1}{c|}{ BIC } \\
\hline \hline AUDJPY 15 min. & 0.079 & 0.099 \\
\hline AUDJPY 1 hora & 3.244 & 3.263 \\
\hline AUDJPY 1 dia & 6.019 & 6.038 \\
\hline CHFJPY 15 min. & 1.435 & 1.454 \\
\hline CHFJPY 1 hora & 2.157 & 2.177 \\
\hline CHFJPY 1 dia & 7.063 & 7.082 \\
\hline EURCHF 15 min. & -10.210 & -10.191 \\
\hline EURCHF 1 hora & -8.148 & -8.128 \\
\hline EURCHF 1 dia & -5.737 & -5.717 \\
\hline EURJPY 15 min. & 1.894 & 1.914 \\
\hline EURJPY 1 hora & 1.200 & 1.219 \\
\hline EURJPY 1 dia & 7.537 & 7.557 \\
\hline GBPJPY 15 min. & 0.850 & 0.870 \\
\hline GBPJPY 1 hora & 6.017 & 6.037 \\
\hline GBPJPY 1 dia & 7.554 & 7.573 \\
\hline
\end{tabular}

Tabela 14 - Valores parciais para a estimativa do expoente de Hurst $(H)$ obtidos por meio das tarefas executadas, conforme descrição no método utilizado, para a relação entre as moedas AUD-JPY, com periodicidade de 1 em 1 dia

\begin{tabular}{|c|c|r|r|r|}
\hline$t$ & $M$ & $R / S_{t}$ & $x=\log \{t\}$ & $y=\log \left\{R / S_{t}\right\}$ \\
\hline \hline 43 & 24 & 16.858010 & 3.761200 & 2.824826 \\
\hline 59 & 17 & 22.225800 & 4.077537 & 3.101254 \\
\hline 79 & 13 & 29.292140 & 4.369448 & 3.377319 \\
\hline 97 & 11 & 35.077250 & 4.574711 & 3.557553 \\
\hline 149 & 7 & 58.272940 & 5.003946 & 4.065138 \\
\hline 199 & 5 & 63.229060 & 5.293305 & 4.146764 \\
\hline 257 & 4 & 86.857240 & 5.549076 & 4.464266 \\
\hline 313 & 3 & 102.258190 & 5.746203 & 4.627501 \\
\hline 773 & 1 & 193.642740 & 6.650279 & 5.266015 \\
\hline
\end{tabular}




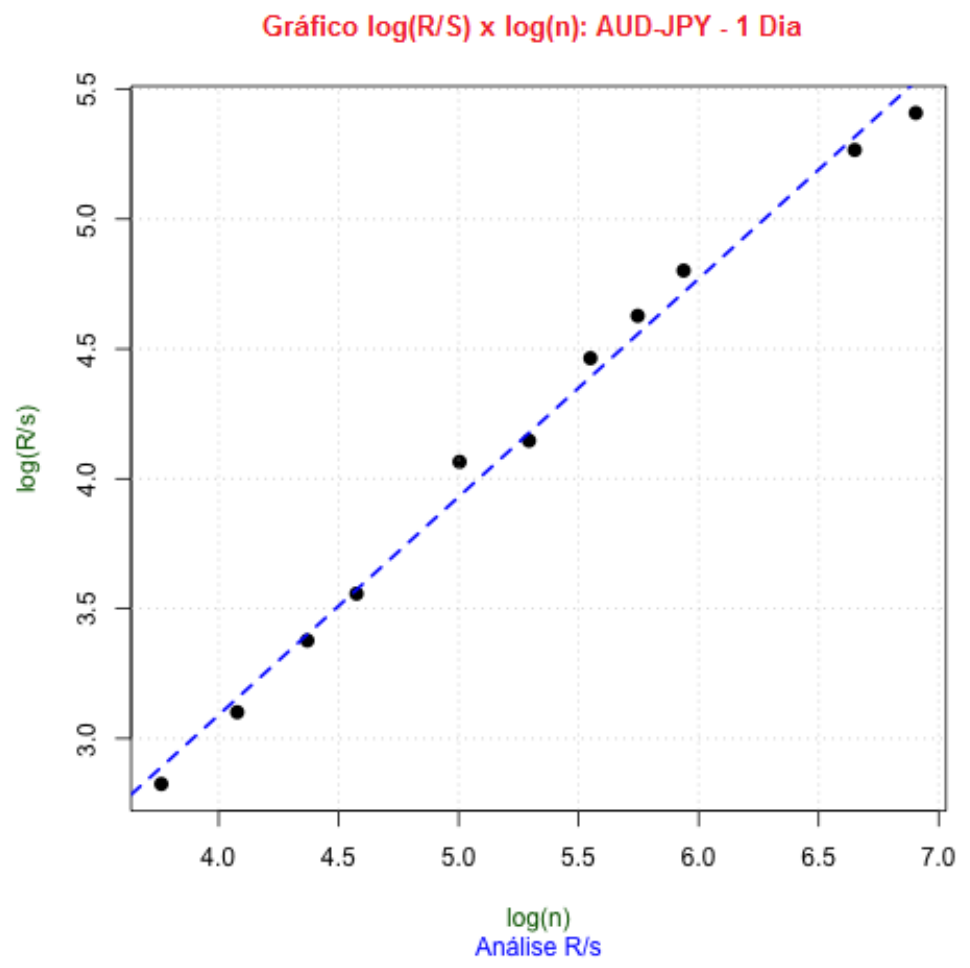

Figura 19 - Gráfico com dispersão dos valores de $\log \left\{R / S_{t}\right\}$ em relação aos valores de $\log \{t\}$, para a relação entre as moedas AUD-JPY, contendo também a reta de tendência.

Tabela 15 - Valores estimados e estatísticas da regressão obtidos por meio das funções $l m$, summary e coef disponíveis no ambiente para análise de dados estatísticos $R$ (47).

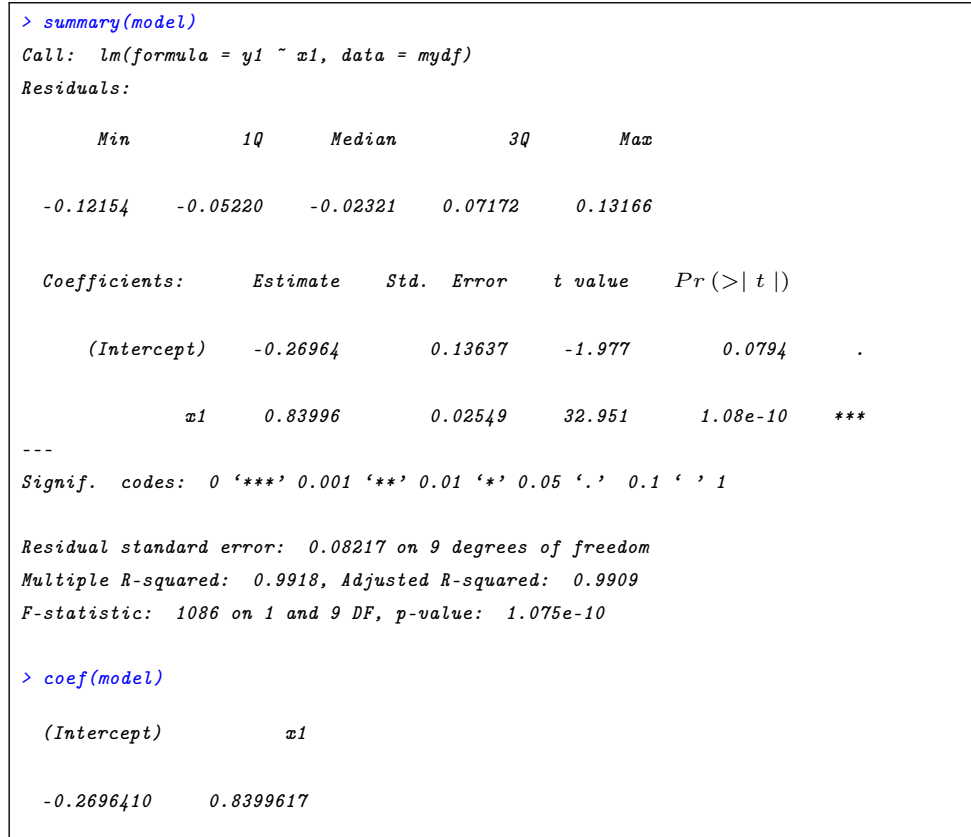




\section{ANÁLISE DOS RESULTADOS}

Conforme a metodologia apresentada, são detalhados os resultados encontrados na aplicação do modelo híbrido SVR-wavelet.

Preliminarmente, usando-se o ambiente de programação para análise de dados estatísticos e gráficos $R$ (47), efetuou-se a eliminação de ruídos contidos nas séries financeiras do tipo FOREX mediante à utilização de técnicas de denoising ou wavelet shrinkage. Depois, após a aplicação da Transformada wavelet MODWT e obtenção dos componentes decompostos de aproximação, $s 5$, e detalhes, $d 1$ a $d 5$, empregou-se, com o auxílio da biblioteca e1071 (90), o método svm em cada componente da Transformada wavelet MODWT, exemplificado pelo uso da componente detalhe $d 1$, conforme:

d1.model $\leftarrow$ svm $(d 1 \sim$ lts, d1.data, scale $=$ TRUE, type $=$ "eps - regression", kernel $="$ radial", gama $=800$, cost $=10000$, tolerance $=0.005$, epsilon $=0.05$, shrinking $=T R U E$, fitted $=T R U E$ )

em que os parâmetros passados como argumento do método svm foram obtidos por intermédio do método tune, também disponível para uso na biblioteca e1071, que ajusta hiperparâmetros de métodos estatísticos usando procedimentos de pesquisa sobre os parâmetros fornecidos.

Em seguida aplicou-se o método predict sobre os modelos calculados, por intermédio do método svm, para se apurar a eficiência do procedimento. A título de exemplo, analisando-se uma das componentes, a componente detalhe $d 5$, Figura 20, é legítimo dizer, pela investigação e exame do gráfico da componente $d 5$, gerada pela Transformada wavelet MODWT, com seus pontos marcados com pequenos círculos $(\bullet)$ pretos, justaposto ao gráfico gerado pelo método predict, marcado com pequenos xis $(x)$ azuis, que eles são congêneres, além do mais, entabulando-se certas métricas chega-se à diferenças muito pequenas, como a maior diferença entre os pontos das séries originais e dos pontos gerados pelo método predict, 0.005383408 , e a $R M S E=0.003482003$.

A Figura 21 exibe o gráfico da série original com seus pontos marcados com pequenos círculos $(\bigcirc)$ azuis, e o gráfico da série predita através do modelo SVR-wavelet híbrido proposto, marcado com pequenos xis $(\times)$ vermelhos em seus pontos, sobreposto sobre a série original para a relação entre as moedas Dólar Australiano (AUD) e o Yen Japonês (JPY) com periodicidade de 15 minutos. 


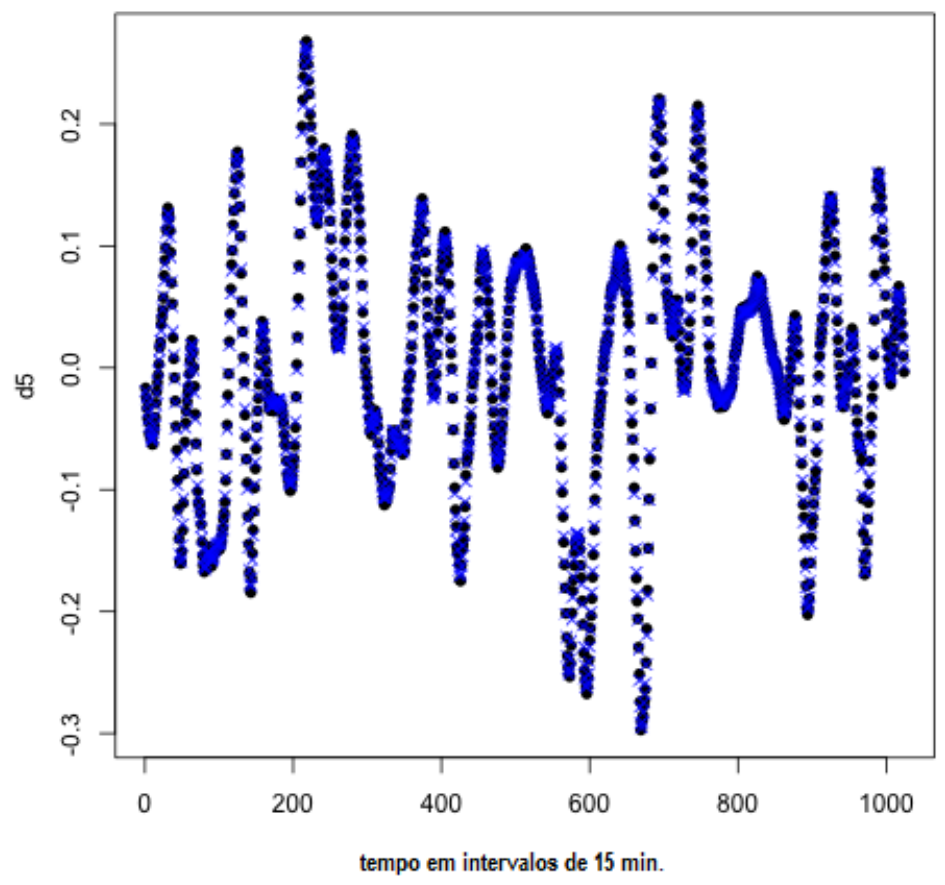

Figura 20 - Gráfico da componente $d 5$, com seus pontos marcados com $(\bullet)$, gerada pela Transformada wavelet MODWT, da relação entre as moedas AUD e JPY com periodicidade de 15 minutos, justaposto ao gráfico gerado pelo método predict, com seus pontos destacados com $(\times)$.

\subsection{SELEÇÃO DO MELHOR MODELO: ARIMA OU SVR-WAVELET HÍBRIDO}

O AIC, tal como o AICc e o BIC, considera o melhor modelo aquele com menores valores, o que pode ser constatado na Tabela 16, comparação dos valores dos métodos AIC, AICc e BIC entre o modelo ARIMA e o modelo híbrido SVR-wavelet.

$\mathrm{Na}$ Tabela 16, as linhas destacadas em verde, com menores valores de AIC, AICc e BIC para o modelo híbrido SVR-wavelet, são a maioria (80\%), enquanto que as linhas destacadas em vermelho, com menores valores de $\mathrm{AIC}$, AICc e BIC para o modelo ARIMA são a minoria (20\%), o que comprova que o modelo baseado em um sistema SVR-wavelet híbrido para previsão de séries temporais financeiras é mais adequado do que aquele modelado por meio do modelo ARIMA.

Alguns testes também foram efetuados antes da aplicação de técnicas de denoising ou wavelet shrinkage para eliminação de ruídos contidos na série. Os resultados obtidos por meio destes testes também mostraram que as séries modeladas através do modelo híbrido SVRwavelet superavam e eram mais adequadas em aproximadamente $73 \%$ do que aquelas séries modelas por meio do modelo ARIMA, com somente $27 \%$ dos casos favoráveis. 


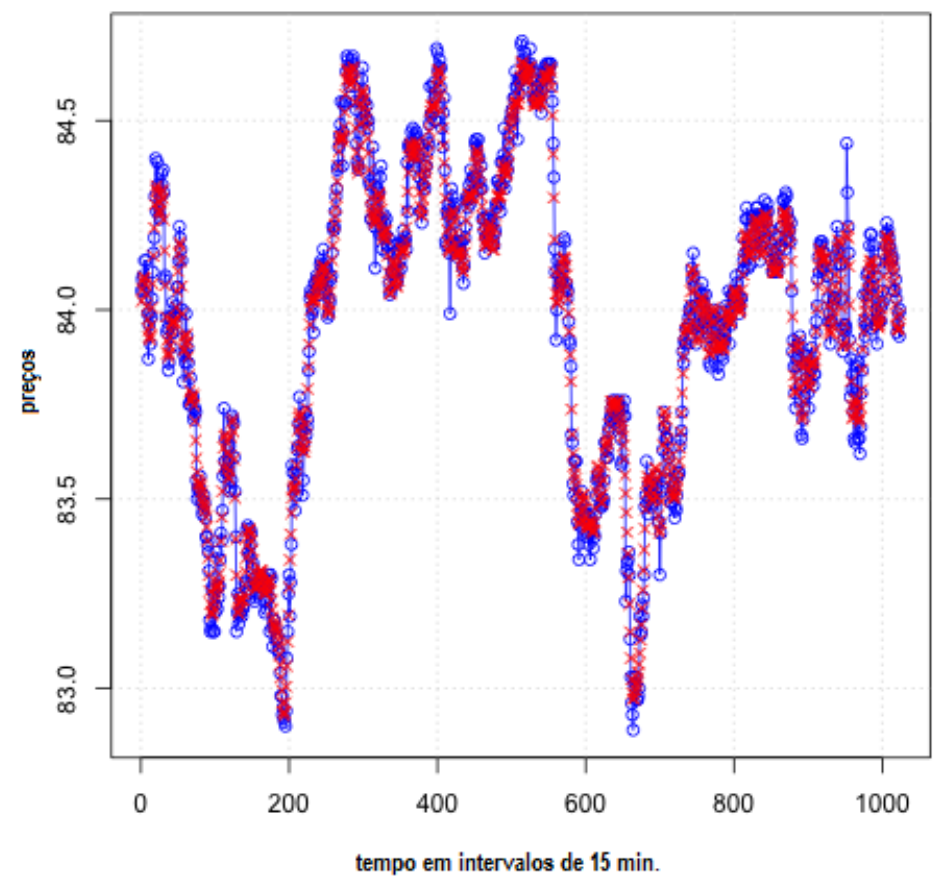

Figura 21 - Gráficos sobrepostos da série original $(\bigcirc)$ e da série predita através do modelo SVR-wavelet híbrido proposto $(\times)$ para a relação entre as moedas AUD e JPY com periodicidade de 15 minutos.

\subsection{SELEÇÃO DO MELHOR MODELO: ARFIMA OU SVR-WAVELET HÍBRIDO}

Como evidenciado nas últimas seções, o AIC, tanto quanto o $\mathrm{AIC}$ e o BIC, pressupõe que o melhor modelo é aquele com os menores valores, o que pode ser apurado pela Tabela 17. comparação dos valores dos métodos AIC, AICc e BIC entre o modelo ARFIMA e o modelo híbrido SVR-wavelet.

$\mathrm{Na}$ Tabela 17, as linhas destacadas em verde, com menores valores de AIC, AICc e BIC para o modelo híbrido SVR-wavelet, novamente são a maioria (80\%), enquanto que as linhas destacadas em vermelho, com menores valores de AIC, AICc e BIC para o modelo ARFIMA são a minoria (20\%), o que corrobora que o modelo baseado em um sistema SVR-wavelet híbrido para previsão de séries temporais financeiras é mais adequado do que aquele modelado por meio do modelo ARFIMA.

De modo similar aos testes com o modelo ARIMA, foram efetuados alguns testes antes da aplicação de técnicas de denoising ou wavelet shrinkage para eliminação de ruídos contidos na série. Os resultados conseguidos por meio destes testes igualmente mostraram que as séries modeladas através do modelo híbrido SVR-wavelet superavam e eram mais adequadas em aproximadamente $93 \%$ do que aquelas séries modelas por meio do modelo ARFIMA, com 
Tabela 16 - Comparação dos valores dos métodos AIC, AICc e BIC entre o modelo ARIMA e o modelo híbrido SVR-wavelet, destacando-se em verde, os menores valores de AIC, AICc e BIC para o modelo híbrido SVR-wavelet.

\begin{tabular}{|c|c|c|c|c|c|c|}
\hline & AIC & AICc & BIC & AIC & AICc & BIC \\
\hline \hline Série & \multicolumn{3}{|c|}{ Modelo ARIMA } & \multicolumn{2}{c|}{ Modelo Híbrido SVR-wavelet } \\
\hline AUDJPY 15 min. & -2601.179 & -2601.155 & -2581.453 & -4334.202 & -4334.178 & -4314.476 \\
\hline AUDJPY 1 hora & -512.137 & -512.113 & -492.411 & -1364.563 & -1364.540 & -1344.837 \\
\hline AUDJPY 1 dia & 2165.019 & 2165.043 & 2184.745 & 999.963 & 999.987 & 1019.689 \\
\hline CHFJPY 15 min. & -1625.353 & -1625.330 & -1605.627 & -2659.319 & -2659.296 & -2639.594 \\
\hline CHFJPY 1 hora & -1478.966 & -1478.942 & -1459.240 & -2432.732 & -2432.709 & -2413.006 \\
\hline CHFJPY 1 dia & 2242.554 & 2242.577 & 2262.280 & 2211.406 & 2211.430 & 2231.132 \\
\hline EURCHF 15 min. & -10549.910 & -10549.890 & -10530.190 & -11267.800 & -11267.770 & -11248.070 \\
\hline EURCHF 1 hora & -10437.360 & -10437.340 & -10417.630 & -3348.369 & -3348.345 & -3328.643 \\
\hline EURCHF 1 dia & -7247.461 & -7247.438 & -7227.736 & 2497.143 & 2497.166 & 2516.869 \\
\hline EURJPY 15 min. & -2049.027 & -2049.003 & -2029.301 & -3324.814 & -3324.791 & -3305.088 \\
\hline EURJPY 1 hora & -2350.538 & -2350.514 & -2330.812 & -3348.369 & -3348.345 & -3328.643 \\
\hline EURJPY 1 dia & 2637.320 & 2637.344 & 2657.046 & 2497.143 & 2497.166 & 2516.869 \\
\hline GBPJPY 15 min. & -2929.129 & -2929.105 & -2909.403 & -3593.484 & -3593.460 & -3573.758 \\
\hline GBPJPY 1 hora & 1567.693 & 1567.717 & 1587.419 & 1496.586 & 1496.609 & 1516.312 \\
\hline GBPJPY 1 dia & 2771.061 & 2771.084 & 2790.786 & 3118.316 & 3118.339 & 3138.042 \\
\hline
\end{tabular}

somente $7 \%$ dos casos favoráveis.

\subsection{SELEÇÃO DO MELHOR MODELO: GARCH OU SVR-WAVELET HÍBRIDO}

Destacados na Tabela 18 estão os valores das métricas AIC e BIC gerados pelo modelo GARCH, através de seus métodos garchFit e predict, e pelo modelo híbrido SVR-wavelet.

$\mathrm{Na}$ Tabela 18, as linhas destacadas em verde, com menores valores de AIC e BIC para o modelo híbrido SVR-wavelet, são mais uma vez o maior número (aproximadamente 67\%), ao passo que as linhas destacadas em vermelho, com menores valores de AIC e BIC para o modelo GARCH são a minoria (aproximadamente 33\%), o que constata que o modelo baseado em um sistema SVR-wavelet híbrido para previsão de séries temporais financeiras é mais adequado do que aquele modelado por meio do modelo GARCH.

De modo análogo aos testes com o modelo ARFIMA, foram efetuados alguns testes antes da aplicação de técnicas de denoising ou wavelet shrinkage para eliminação de ruídos contidos 
Tabela 17 - Comparação dos valores dos métodos AIC, AICc e BIC entre o modelo ARFIMA e o modelo híbrido SVR-wavelet, destacando-se em verde, os menores valores de AIC, AICc e BIC para o modelo híbrido SVR-wavelet.

\begin{tabular}{|c|c|c|c|c|c|c|}
\hline & AIC & AICc & BIC & AIC & AICc & BIC \\
\hline \hline Série & \multicolumn{3}{|c|}{ Modelo ARFIMA } & \multicolumn{2}{c|}{ Modelo Híbrido SVR-wavelet } \\
\hline AUDJPY 15 min. & -2605.580 & -2605.556 & -2585.854 & -4334.202 & -4334.178 & -4314.476 \\
\hline AUDJPY 1 hora & -170.112 & -170.089 & -150.387 & -1364.563 & -1364.540 & -1344.837 \\
\hline AUDJPY 1 dia & 2313.003 & 2313.026 & 2332.729 & 999.963 & 999.987 & 1019.689 \\
\hline CHFJPY 15 min. & -1625.507 & -1625.483 & -1605.781 & -2659.319 & -2659.296 & -2639.594 \\
\hline CHFJPY 1 hora & -1077.437 & -1077.414 & -1057.712 & -2432.732 & -2432.709 & -2413.006 \\
\hline CHFJPY 1 dia & 2582.415 & 2582.439 & 2602.141 & 2211.406 & 2211.430 & 2231.132 \\
\hline EURCHF 15 min. & -10580.170 & -10580.150 & -10560.450 & -11267.800 & -11267.770 & -11248.070 \\
\hline EURCHF 1 hora & -10441.610 & -10441.590 & -10421.880 & -3348.369 & -3348.345 & -3328.643 \\
\hline EURCHF 1 dia & -7226.452 & -7226.428 & -7206.726 & 2497.143 & 2497.166 & 2516.869 \\
\hline EURJPY 15 min. & -1867.270 & -1867.246 & -1847.544 & -3324.814 & -3324.791 & -3305.088 \\
\hline EURJPY 1 hora & -2220.354 & -2220.330 & -2200.628 & -3348.369 & -3348.345 & -3328.643 \\
\hline EURJPY 1 dia & 2898.977 & 2899.000 & 2918.703 & 2497.143 & 2497.166 & 2516.869 \\
\hline GBPJPY 15 min. & -2565.099 & -2565.076 & -2545.373 & -3593.484 & -3593.460 & -3573.758 \\
\hline GBPJPY 1 hora & 2126.523 & 2126.547 & 2146.249 & 1496.586 & 1496.609 & 1516.312 \\
\hline GBPJPY 1 dia & 3091.113 & 3091.137 & 3110.839 & 3118.316 & 3118.339 & 3138.042 \\
\hline
\end{tabular}

na série. Todavia os resultados conseguidos por meio destes teste foram os mesmos, ou seja as séries modeladas através do modelo híbrido SVR-wavelet superaram e foram mais adequadas em aproximadamente $67 \%$ do que aquelas séries modelas por meio do modelo GARCH, com somente $33 \%$ dos casos favoráveis.

\subsection{SELEÇÃO DO MELHOR MODELO: SVR TRADICIONAL COM KERNEL OU SVR- WAVELET HÍBRIDO}

Como demonstrado pelas seções precedentes, o $\mathrm{AIC}$, tanto quanto o $\mathrm{AIC}$ e o $\mathrm{BIC}$, admite que o modelo mais razoável é aquele com os menores valores, o que pode ser averiguado pela Tabela 19, comparação dos valores dos métodos AIC, AICc e BIC entre o modelo SVR tradicional com Kernel e o modelo híbrido SVR-wavelet.

$\mathrm{Na}$ Tabela 19, as linhas marcadas em verde, com menores valores de AIC, AICc e BIC para o modelo híbrido SVR-wavelet, novamente são a maioria $80 \%$, enquanto que as linhas marcadas 
Tabela 18 - Comparação dos valores dos métodos AIC e BIC entre o modelo GARH e o modelo híbrido SVR-wavelet, destacando-se em verde, os menores valores de AIC e BIC para o modelo híbrido SVR-wavelet.

\begin{tabular}{|c|c|c|c|c|c|c|}
\hline & AIC & AICc & BIC & AIC & AICc & BIC \\
\hline \hline Série & \multicolumn{2}{|c|}{ Modelo GARCH } & \multicolumn{2}{c|}{ Modelo Hibrido SVR-wavelet } \\
\hline AUDJPY 15 min. & 0.079 & & 0.099 & -4334.202 & -4334.178 & -4314.476 \\
\hline AUDJPY 1 hora & 3.244 & & 3.263 & -1364.563 & -1364.540 & -1344.837 \\
\hline AUDJPY 1 dia & 6.019 & & 6.038 & 999.963 & 999.987 & 1019.689 \\
\hline CHFJPY 15 min. & 1.435 & & 1.454 & -2659.319 & -2659.296 & -2639.594 \\
\hline CHFJPY 1 hora & 2.157 & & 2.177 & -2432.732 & -2432.709 & -2413.006 \\
\hline CHFJPY 1 dia & 7.063 & & 7.082 & 2211.406 & 2211.430 & 2231.132 \\
\hline EURCHF 15 min. & -10.210 & & -10.191 & -11267.800 & -11267.770 & -11248.070 \\
\hline EURCHF 1 hora & -8.148 & & -8.128 & -3348.369 & -3348.345 & -3328.643 \\
\hline EURCHF 1 dia & -5.737 & & -5.717 & 2497.143 & 2497.166 & 2516.869 \\
\hline EURJPY 15 min. & 1.894 & & 1.914 & -3324.814 & -3324.791 & -3305.088 \\
\hline EURJPY 1 hora & 1.200 & & 1.219 & -3348.369 & -3348.345 & -3328.643 \\
\hline EURJPY 1 dia & 7.537 & & 7.557 & 2497.143 & 2497.166 & 2516.869 \\
\hline GBPJPY 15 min. & 0.850 & & 0.870 & -3593.484 & -3593.460 & -3573.758 \\
\hline GBPJPY 1 hora & 6.017 & & 6.037 & 1496.586 & 1496.609 & 1516.312 \\
\hline GBPJPY 1 dia & 7.553 & & 7.573 & 3118.316 & 3118.339 & 3138.042 \\
\hline
\end{tabular}

em vermelho, com menores valores de AIC, AICc e BIC para o modelo SVR tradicional com Kernel são a minoria $20 \%$, o que ratifica que o modelo baseado em um sistema SVR-wavelet híbrido para previsão de séries temporais financeiras é mais apropriado do que aquele modelado por meio do modelo SVR tradicional com Kernel.

De maneira equivalente aos testes com o modelo GARCH, foram efetuados alguns testes antes da aplicação de técnicas de denoising ou wavelet shrinkage para eliminação de ruídos contidos na série. Os resultados atingidos por meio destes testes semelhantemente mostraram que as séries modeladas através do modelo híbrido SVR-wavelet superavam e eram mais adequadas em aproximadamente $60 \%$ do que aquelas séries modelas por meio do modelo SVR tradicional com Kernel, com somente $40 \%$ dos casos favoráveis.

\subsection{CONCLUSÕES SOBRE A ADERÊNCIA AO EXPOENTE DE HURST}

A Tabela 20 sumariza o teste de significância, conforme sugerido por Couillard e Davison, utilizando-se da estatística $p$-value $<0,001$. Estes valores foram obtidos por meio da função 
Tabela 19 - Comparação dos valores dos métodos AIC, AICc e BIC entre o modelo SVR tradicional com Kernel e o modelo híbrido SVR-wavelet, destacando-se em verde, os menores valores de AIC, AICc e BIC para o modelo híbrido SVR-wavelet.

\begin{tabular}{|c|c|c|c|c|c|c|}
\hline & AIC & AICc & BIC & AIC & AICc & BIC \\
\hline \hline Série & Modelo SVR tradicional com Kernel & \multicolumn{2}{|c|}{ Modelo Hibrido SVR-wavelet } \\
\hline AUDJPY 15 min. & -3255.428 & -3255.404 & -3235.702 & -4334.202 & -4334.178 & -4314.476 \\
\hline AUDJPY 1 hora & -819.089 & -819.065 & -799.363 & -1364.563 & -1364.540 & -1344.837 \\
\hline AUDJPY 1 dia & 1779.209 & 1779.232 & 1798.935 & 999.963 & 999.987 & 1019.689 \\
\hline CHFJPY 15 min. & -1662.520 & -1662.496 & -1642.794 & -2659.319 & -2659.296 & -2639.594 \\
\hline CHFJPY 1 hora & -2034.998 & -2034.975 & -2015.272 & -2432.732 & -2432.709 & -2413.006 \\
\hline CHFJPY 1 dia & 2367.879 & 2367.903 & 2387.605 & 2211.406 & 2211.430 & 2231.132 \\
\hline EURCHF 15 min. & -10335.920 & -10335.890 & -10316.190 & -11267.800 & -11267.770 & -11248.070 \\
\hline EURCHF 1 hora & -10490.080 & -10490.060 & -10470.360 & -3348.369 & -3348.345 & -3328.643 \\
\hline EURCHF 1 dia & -7059.529 & -7059.506 & -7039.803 & 2497.143 & 2497.166 & 2516.869 \\
\hline EURJPY 15 min. & -2551.386 & -2551.363 & -2531.660 & -3324.814 & -3324.791 & -3305.088 \\
\hline EURJPY 1 hora & -2702.717 & -2702.694 & -2682.991 & -3348.369 & -3348.345 & -3328.643 \\
\hline EURJPY 1 dia & 2506.178 & 2506.202 & 2525.904 & 2497.143 & 2497.166 & 2516.869 \\
\hline GBPJPY 15 min. & -3418.001 & -3417.978 & -3398.275 & -3593.484 & -3593.460 & -3573.758 \\
\hline GBPJPY 1 hora & 1497.892 & 1497.915 & 1517.617 & 1496.586 & 1496.609 & 1516.312 \\
\hline GBPJPY 1 dia & 2917.440 & 2917.463 & 2937.166 & 3118.316 & 3118.339 & 3138.042 \\
\hline
\end{tabular}

t.test (Student's t-Test), disponível nas bibliotecas padrões do ambiente para análise de dados estatísticos $R$ (47).

\subsubsection{Resultados obtidos dos cálculos do Expoente de Hurst}

A Tabela 21 sintetiza os resultados obtidos dos cálculos do Expoente de Hurst por meio dos funções desenvolvidas no ambiente para análise de dados estatísticos $R$ (47), conforme metodologia desenvolvida por Mandelbrot e Wallis (85), baseada nos trabalhos de Hurst (62).

Conforme destacou Mandelbrot (85), que desenvolveu seus estudos baseados nos trabalhos de Hurst (62), um movimento Browniano tem $H=0.5$, enquanto que um processo persistente tem $H>0.5$ e um processo anti-persistente tem $H<0.5$. Portanto, pela Tabela 21, é possível verificar que as séries históricas de preços das paridades entre as moedas apresentadas nesta Tabela se mostram como processos persistentes, ou seja, $H>0.5$.

Um outro fato destacado na Tabela 21. contendo os resultados sumarizados, é que o Ex- 
Tabela 20 - Teste de significância, conforme sugerido por Couillard e Davison, utilizando-se da estatística $p$-value $<0,001$, obtidos por meio da função t.test disponível no ambiente para análise de dados estatísticos $R$ (47).

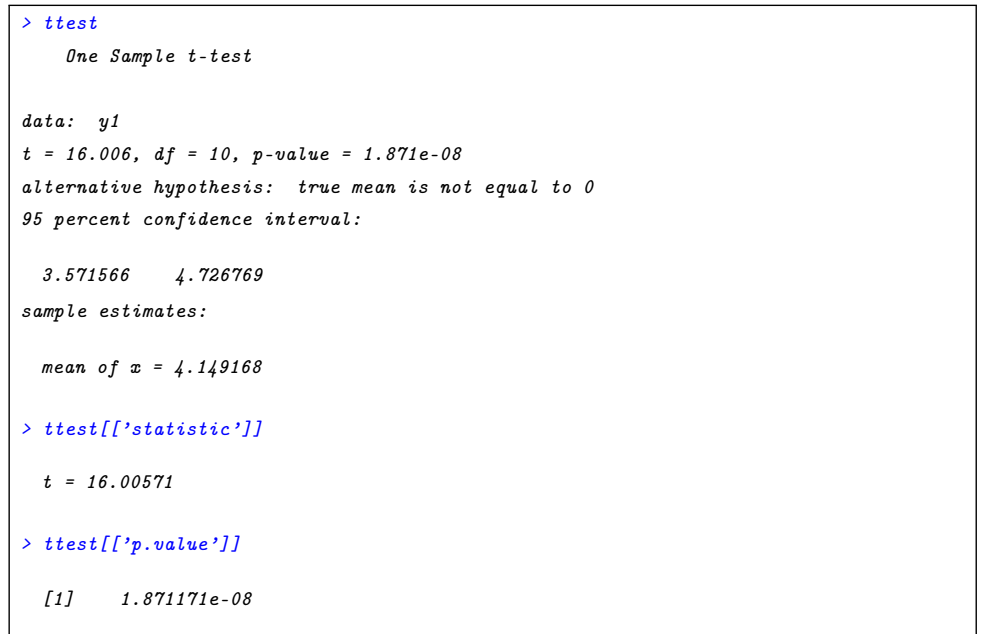

poente de Hurst ficou constante entre as periodicidades de 1 dia, 1 hora e 15 minutos, o que mostra que o Expoente de Hurst pode ser considerado válido para as séries históricas apresentadas nesta Tabela. 
Tabela 21 - Resultados obtidos por meio das funções desenvolvidas no ambiente para análise de dados estatísticos $R$ (47), conforme metodologia desenvolvida por Mandelbrot e Wallis (85), baseada nos trabalhos de Hurst (62).

\begin{tabular}{|c|c|c|c|c|}
\hline Série & Hurst & (Intercept) & t-statistic & p.value \\
\hline \hline AUDJPY 15 min. & 0.78944730 & -0.02336953 & 16.77667 & $1.18723 e-08$ \\
\hline AUDJPY 1 hora & 0.8324442 & -0.2153603 & 16.13222 & $1.73416 e-08$ \\
\hline AUDJPY 1 dia & 0.8399617 & -0.2696410 & 16.00571 & $1.871171 e-08$ \\
\hline CHFJPY 15 min. & 0.8534669 & -0.3911333 & 15.48972 & $2.566635 e-08$ \\
\hline CHFJPY 1 hora & 0.8184394 & -0.1341707 & 16.29031 & $1.578194 e-08$ \\
\hline CHFJPY 1 dia & 0.8545995 & -0.3065525 & 15.80562 & $2.112716 e-08$ \\
\hline EURCHF 15 min. & 0.8954869 & -0.6836513 & 14.46114 & $4.966747 e-08$ \\
\hline EURCHF 1 hora & 0.8845434 & -0.5429375 & 14.60365 & $4.521147 e-08$ \\
\hline EURCHF 1 dia & 0.7519774 & 0.1453708 & 17.09724 & $9.882615 e-09$ \\
\hline EURJPY 15 min. & 0.8285461 & -0.2140661 & 16.21255 & $1.652901 e-08$ \\
\hline EURJPY 1 hora & 0.78266634 & 0.03470801 & 16.91379 & $1.097201 e-08$ \\
\hline EURJPY 1 dia & 0.8545435 & -0.2964951 & 15.86572 & $2.036754 e-08$ \\
\hline GBPJPY 15 min. & 0.8529881 & -0.3298242 & 15.61343 & $2.377259 e-08$ \\
\hline GBPJPY 1 hora & 0.8781442 & -0.4207742 & 15.42134 & $2.678384 e-08$ \\
\hline GBPJPY 1 dia & 0.8567473 & -0.3236433 & 15.79052 & $2.132282 e-08$ \\
\hline
\end{tabular}




\section{CONCLUSÃO}

Neste capítulo são apresentados os resultados obtidos e as contribuições alcançadas, uma análise geral do trabalho, e sugestões para trabalhos futuros.

\subsection{RESULTADOS OBTIDOS E CONTRIBUIÇÕES ALCANÇADAS}

A previsão de dados futuros não mais reside no domínio de matemáticos e estatísticos e vem se tornando um assunto interdisciplinar. Atualmente, diversas áreas do conhecimento vêm adotando completamente a metodologia de previsão de séries temporais não-lineares e não-estacionárias como parte de suas operações primárias.

A previsão de séries temporais financeiras do tipo FOREX, não-lineares e não-estacionárias, tem sido um estímulo para utilização de sistemas híbridos aplicando aprendizagem de máquinas e a teoria da aprendizagem estatística. As técnicas de previsão clássica, incluindo os modelos matemáticos clássicos, desenvolvidas para processos lineares são inconvenientes quando se trata de dados de séries temporais não-lineares e não-estacionárias.

Neste estudo, destacou-se alguns dos mais relevantes métodos utilizados para regressão, como os modelos matemáticos tradicionalmente utilizados como regressores de séries temporais financeiras, e de aprendizado de máquinas, particularmente ao SVR, regressão por meio de Máquinas de Suporte Vetorial (SVM), e à Transformada wavelet, como alternativas adequadas para previsão de dados não-lineares e não-estacionárias.

Em um esforço para compreender as limitações de cada tipo de modelo e seus trade-offs este estudo efetuou uma análise crítica frente às diversas iniciativas de pesquisa acadêmicas desenvolvidas mediante a aplicação de métodos de aprendizado de máquinas na regressão de séries temporais.

Também neste estudo, evidenciou-se um novo modelo de previsão, retratado por modelos híbridos, em particular o modelo de previsão por meio do sistema SVR-wavelet, que integra modelos wavelets e SVR na previsão dos dados das séries temporais financeiras do tipo FOREX.

O modelo híbrido SVR-wavelet manifestou ser capaz e eficiente na previsão dos dados das séries temporais financeiras do tipo FOREX. Seu desempenho, quando comparado com outros modelos, tais como os modelos matemáticos tradicionais, mostrou-se significativamente melhor na previsão de séries temporais não-lineares e não-estacionárias (próximo de 67\% na pior predição e igual a 80\% na melhor predição). Algumas medidas de desempenho, tais como MSE, RMSE, MAE, MPE, MAPE e MASE também evidenciaram que o modelo híbrido SVR-wavelet superou os outros modelos tradicionais, tais como os modelos ARIMA, ARFIMA, GARCH e o modelo SVR tradicional com Kernel. Comprovou-se também que após a eliminação de ruídos 
contidos na série, pela aplicação de técnicas de denoising ou wavelet shrinkage, os resultados de desempenho encontrados, comparando-se os modelos ARIMA, ARFIMA, GARCH e o modelo SVR tradicional com Kernel com o modelo híbrido SVR-wavelet, mostraram-se consistentes entre as séries AUD-JPY, CHF-JPY, EUR-CHF, EUR-JPY e GBP-JPY, com periodicidades de 15 minutos, 1 hora e 1 dia.

Similarmente neste estudo, integrou-se modelos wavelets e SVR culminando em um novo modelo híbrido para se prever dados não-lineares de séries temporais não-estacionárias, como as séries Financeiras do tipo FOREX. Depois de combinar essas duas abordagens inovadoras e aplicá-las em um novo modelo de predição, conseguiu-se uma predição robusta, como verificado pelas métricas AIC, AICc e BIC, comparando-a com os métodos tradicionais e com o modelo SVM padrão, executado sem a adição de wavelet.

As séries financeiras do tipo FOREX também se mostraram aderentes ao Expoente de Hurst e foi possível verificar que as séries históricas de preços das paridades entre as moedas, apresentadas neste trabalho, se mostraram como processos persistentes.

Entretanto a previsão de séries temporais não-lineares e não-estacionárias ainda precisa de consideráveis pesquisas acadêmicas para garantir que se atinja a maturidade e possa dispor de todo o seu potencial.

Em síntese, esta pesquisa produziu resultados satisfatórios, e cumpriu seu objetivo em conceituar um novo modelo híbrido de previsão por meio do sistema SVR-wavelet, integração de modelos wavelets e SVR, na previsão dos dados das séries temporais financeiras do tipo FOREX.

Ao final desta pesquisa e trabalho realizados, acredito que ainda há espaço para pesquisa e melhoria da precisão da previsão do modelo híbrido proposto. Desejo que esta tese incentive outros interesses em pesquisas sobre esse assunto, que o modelo de previsão estudado por este trabalho seja melhorado e que também possa ser aplicado para resolver questões fora do mundo acadêmico.

\subsection{SUGESTÕES PARA TRABALHOS FUTUROS}

Como considerações finais, após a análise dos resultados, alguns temas e assuntos pesquisados podem completar ou ainda aumentar as contribuições deste trabalho. Seguem os assuntos:

Há uma variedade de séries temporais financeiras que podem servir como casos de uso do modelo híbrido proposto e da metodologia apresentada neste trabalho para previsão de séries temporais genéricas, tais como séries do Dólar Futuro, do Índice BOVESPA, de preços de commodities como açúcar, café, bezerro, entre tantas outras séries existentes no Mercado 
Financeiro.

A Aprendizagem Profunda (DL), é um paradigma para a realização de aprendizagem de máquina, e a tecnologia tornou-se um tema da atualidade devido aos resultados inigualáveis em aplicações como visão computacional, reconhecimento de fala e compreensão de linguagem natural. Desse modo estudos comparativos entre modelos híbridos empregando modelos de Aprendizagem Profunda (DL) podem ser temas interessantes de pesquisas futuras.

Os padrões gráficos, utilizados pela Análise Técnica, fundamentados na força de movimentos entre os compradores e os vendedores, delineiam tendências. Pelos padrões dos gráficos, usualmente chamados na Área Financeira de Candlestick, é possível prever o comportamento subsequente de preços admitindo a existência da repetição de figuras, consequentemente com possibilidade de previsão de preços futuros. À vista disso, estudos destes padrões gráficos, por meio de modelos híbridos, aparecem como temas relevantes de investigações futuras.

Segundo o que foi apresentado no Capítulo 6, Ambiente de Programação para Análise de Dados Estatísticos e Gráficos R, para o escopo deste trabalho somente parte dos parâmetros foram utilizados para os cálculos do modelo híbrido proposto. Todavia existem uma variedade de outros parâmetros que podem ser melhores estudados em futuras pesquisas.

O cálculo estimado do Expoente de Hurst $(H)$, exposto por este trabalho, abordou uma das propriedades deste índice. A suposição da existência de um Mercado Fractal, pela hipótese de que os Mercados Eficientes possuem alguma anomalia, destacado por alguns autores apontados neste trabalho, serve como incentivo a novas pesquisas, especialmente pela possibilidade de sua aplicação por intermédio de aprendizagem de máquina. 


\section{Referências}

1 Carlos Aguiló. Cambios significativos en el mundo empresarial. Economía Industrial, Vol. $\mathrm{VI}($ no. 330):308, 1999.

2 B. W. Ambrose, E. W. Ancel, and M. D. Griffiths. Fractal structure in the capital markets revisited. Financial Analysts Journal, Vol. 49:pp. 73-77, 1993.

$3 \mathrm{H}$. Arsham. Kuiper's p-value as a measuring tool and decision procedure for the goodnessof-fit test. Journal of Applied Statistics, Vol. 15(no. 2):pp. 131-135, 2006.

4 A. Aussem, J. Campbell, and F. Murtagh. Wavelet-based feature extraction and decomposition strategies for financial forecasting. Journal of Computational Intelligence in Finance, Vol. 6(no. 2):pp. 5-12, 1998.

5 J. Barkoulas, W. C. Labys, and J. Onochie. Fractional dynamics in international commodity prices. Journal of Futures Markets, Vol. 17:pp. 161-189, 1997.

6 L. C. Barroso, M. M. A. Barroso, F. F. Campos, M. L. B. Carvalho, and M. L. Maia. Cálculo Numérico. 2a edition, 1987.

7 D. Basak, S. Pal, and D. C. Patranabis. Support vector regression. Neural Information Processing - Letters and Reviews, Vol. 11(no. 10), 2007.

8 J. C. Berg. Wavelets in physics. Cambridge University Press, 2004.

9 B. Bhattacharya, K. Mukherjee, and G. Toussaint. Geometric decision rules for instancebased learning problems. Lecture Notes in Computer Science (including subseries Lecture Notes in Artificial Intelligence and Lecture Notes in Bioinformatics), Vol. 3776:pp. 60-69, 2005.

10 B. K. Bhattacharya, R. S. Poulsen, and G. T. Toussaint. Application of proximity graphs to editing nearest neighbor decision rule. International Symposium on Information Theory, pages 1-25, 1992.

11 L. Bódis. Financial Time Series Forecasting Using Artificial Neural Networks. PhD thesis, Babes-Bolyai University, 2004.

12 D. Bonesso. Estimação dos parâmetros do kernel em um classificador svm na classificação de imagens hiperespectrais em uma abordagem multiclasse. UFRGS - Centro Estadual de Sensoriamento Remoto e Metrologia - Programa de Pós-Graduação em Sensoriamento Remoto, 2013. 
13 F. A. Botelho. Análise Técnica e estratégia Operacional. Enfoque Gráfico, São Paulo, 1a edition, 2004.

14 G. E. P. Box, G. M. Jenkins, and G. C. Reinsel. Time Series Analysis: Forecasting and control. 4th edition, 1994.

$15 \mathrm{H}$. Bozdogan. Model selection and akaike's information criterion (aic): The general theory and its analytical extensions. Psychometrika, Vol. 52(no. 3):pp. 345-370, 1987.

16 C. J. C. Burges. A tutorial on support vector machines for pattern recognition. Knowledge Discovery and Data Mining, Vol. 43:pp. 1-43, 1998.

17 C. Campbell. An introduction to kernel methods kernel methods. Studies in Fuzziness and Soft Computing, Vol. 66:pp. 155-192, 2001.

18 J. Y. Campbell, A. W. Lo, and A. C. MacKinlay. The Econometrics of Financial Markets. Princeton University Press, 1st edition, 1997.

19 L. J. Cao and F. H. Tay. Support vector machine with adaptive parameters in financial time series forecasting. IEEE transactions on neural networks / a publication of the IEEE Neural Networks Council, Vol. 14(no. 6):pp. 1506-1518, 2003.

20 J. Chambers, Cleveland W., Kleiner B., and Tukey P. Graphical methods for data analysis. Wadsworth, 1983.

$21 \mathrm{~K}$. Y. Chen and C. H. Wang. A hybrid sarima and support vector machines in forecasting the production values of the machinery industry in taiwan. Expert Systems with Applications, Vol. 32(no. 1):pp. 254-264, 2007.

22 C. K. Chui. An introduction to wavelets. Boston, 1992.

23 W. Constantine and D. Percival. wmtsa: Wavelet methods for time series analysis. CRAN, 2016.

$24 \mathrm{H}$. Cootner. The random character of stock market prices. MIT Press, 1964.

25 M. Corazza, A. G. Malliaris, and C. Nardelli. Searching for fractal structure in agricultural futures markets. Journal of Futures Markets, Vol. 17:pp. 433-473, 1997.

26 C. R. Cornish, C. S. Bretherton, and D. B. Percival. Maximal overlap wavelet statistical analysis with application to atmospheric turbulence. Boundary-Layer Meteorology, Vol. 119(no. 2):pp. 339-374, 2006. 
27 I. J. Costa and Vargas J. Análise fundamentalista e análise técnica: Agregando valor a uma carteira de ações. DESTARTE, Vol. 1(no. 1), 2011.

28 N. S. S. Costa. Detecção de quebras estruturais em séries temporais: Implementação dos testes de shimotsu com uma aplicação em séries do mercado de câmbio. Master's thesis, Universidade de Brasília - Departamento de Estatística, 2016.

29 M. Couillard and M. Davison. A comment on measuring the hurst exponent of financial time series. Physica A: Statistical Mechanics and its Applications, Vol. 348(no. 348):pp. 404-418, 2005.

30 I. Daubechies. Orthonormal bases of compactly supported wavelets. Communications on Pure and Applied Mathematics, Vol. 41:pp. 909-996, 1988.

31 I. Daubechies, S. Mallat, and A. Willsky. Introduction to the special issue on wavelet transforms and multiresolution signal analysis, volume Vol. 2, pages 528-532. 1992.

32 T. Di Matteo, T. Aste, and M. M. Dacorogna. Long term memories of developed and emerging markets: Using the scalinganalysis to characterize their stage of development. J. Bank. Financ., Vol. 29:pp. 827-851, 2005.

33 S. Dietz. Autoregressive neural network processes univariate, multivariate and cointegrated models with application to the germa automobile industry. Wirtschaftswissenschaften Universität Passau, 2010.

34 Z. Y. Dong, T. K. Saha, and K. P. Wong. Business applications and computational intelligence. Group, Idea, pages 131-154, 2005.

35 Paulo César Emiliano. Fundamentos e aplicações dos critérios de informação: Akaike e bayesiano. Universidade Federal de Lavras, 2009.

36 E. F. Fama. The behavior of stock-market prices. The Journal of Business, Vol. 38:p. 34, 1965.

37 F. Family. Book review: Fractal growth phenomena. Journal of Statistical Physics, Vol. 66:pp. 683-686, 1992.

38 A. A. Farag and R. M. Mohamed. Regression using support vector machines: Basic foundations. University of Louisville - Electrical and Computer Engineering - Department Computer Vision and Image Processing Laboratory, 2004.

39 A. B. Favaretto. Estimativa do expoente de hurst de séries temporais de chuvas do estado de são paulo usando as transformadas de fourier, wavelets e análise $\mathrm{r} / \mathrm{s}$. UNESP, Instituto de Geociências e Ciências Exatas, 2004. 
40 D. Fay and J. Ringwood. A wavelet transfer model for time series forecasting. International Journal of Bifurcation and Chaos, Vol. 17(no. 10):pp. 3691-3696, 2007.

41 F. Folger and Leibfarth L. Make Money Trading: How to Build a Winning Trading Business with foreword. Marketplace Books, Inc., Columbia, MD, 1st edition, 2007.

42 A. A. Freitas. A survey of evolutionary algorithms for data mining and knowledge discovery. Curitiba, 2003.

43 A. A. Freitas. A review of evolutionary algorithms for data mining. Canterbury, 2007.

44 Michael Friendly and Daniel Denis. The early origins and development of the scatterplot. Journal of the History of the Behavioral Sciences, Vol. 41(2):pp. 103-130, 2005.

45 Y. Fu. Distributed data mining: an overview. IEEE Technical Committee on Distributed Processing newsletter, 2001.

46 R. Gencay, F. Selcuk, and B. Whitcher. An introduction to wavelets and other filtering methods in finance and economics. Academic Press, 2001.

47 R. Gentleman and R. Ihaka. The $r$ foundation for statistical computing, 2017.

48 A. Ghosh and Freitas A. A. Guest editorial: Data mining and knowledge discovery with evolutionary algorithms. Springer, 2003.

49 C. L. Giles, S. Lawrence, and A. C. Tsoi. Noisy time series prediction using a recurrent neural network and grammatical inference. Machine Learning, Vol. 44(no. 1):pp. 161-183, 2001.

50 D. Glassman. The foreign exchange market. Journal of International Economics, Vol. 30(no. 3-4):pp. 385-387, 1991.

51 C. W. J. Granger and R. Joyeux. An introduction to long memory time series models and fractional differencing. Journal of Time Series Analysis, Vol. 1(no. 1):pp. 15-29, 1980.

52 O. C. Guarnieri. Um Estudo Empírico da Eficiência da Análise Técnica como Instrumento na Predição do Comportamento dos Preços das Ações: O Caso Embraer. PhD thesis, Universidade de Taubaté, 2006.

$53 \mathrm{~K}$. Hadri and Y. Rao. Kpss test and model misspecifications. Applied Economics Letters, Vol. 16(no. 12):pp. 1187-1190, 2009.

54 R. Hahn. R formula notation tutorial. Econometrics and Statistics Group - University of Chicago Booth School of Business - Business Statistics 41000, 2017. 
55 J. Han and M. Kamber. Data Mining: Concepts and Techniques. Morgan Kaufmann Publishers, 2nd. ed., 2006.

56 S. Haykin. Neural Networks - A Compreensive Foundation. Prentice-Hall, New Jersey, 2nd edition, 1999.

57 S. Haykin. Redes neurais: princípios e prática. Bookman, 2001.

58 M. A. Hearst, B. Scholkopf, S. Dumais, E. Osuna, and J. Platt. Trends and controversies - support vector machines. IEEE Intelligent Systems, Vol. 13(no. 4):pp. 18-28, 1998.

59 A. Hejlsberg. Visual studio .net framework 4.6.2, 2016.

60 R. Herbrich. Learning kernel classifiers: Theory and algorithms. MIT Press, 2001.

61 J. R. M. Hosking. Fractional differencing. Biometrika, Vol. 68(no. 1):pp. 165-176, 1981.

$62 \mathrm{H}$. E. Hurst. Long-term storage capacity of reservoirs. Transactions of the American Society of Civil Engineers, Vol. 116(no. 1):pp. 770-799, 1951.

63 R. Hyndman, M. O'Hara-Wild, C. Bergmeir, S. Razbash, and Wang E. Forecast: Forecasting functions for time series and linear models. CRAN, 2017.

64 L. Jäntschi and S. D. Bolboaca. Distribution fitting 2. pearson-fisher, kolmogorov-smirnov, anderson-darling, wilksshapiro, cramer-von-misses and jarque-bera statistics. Technical University of Cluj-Napoca, 400641 Cluj-Napoca, 2009.

65 B. Jawerth and W. Sweldens. An overview of wavelet based multiresolution analyses, volume Vol. 36, pages 377-412. 1994.

66 J. Kamruzzaman and R. A. Sarker. Forecasting of currency exchange rates using ann: a case study. International Conference on Neural Networks and Signal Processing, 2003. Proceedings of the 2003, Vol. 1(no. 1):pp. 793-797, 2003.

67 G. Kristopher. A guide to technical indicators, dow theory, and elliott wave theory. Market Realist, 2014.

68 Massaroppe L. Estimação da causalidade de Granger no caso de interação não-linear. PhD thesis, Escola Politécnica - Electronic Systems, 2016.

69 M. Larrain. Testing chaos and nonlinearities in t-bill rates. Financial Analysts Journal, Vol. 47:pp. 51-62, 1991. 
70 T.-H. Lee. Neural network test and nonparametric kernel test for neglected nonlinearity in regression models. Department of Economics University of California, 2000.

71 T. P. Lemke and G. T. Lins. Soft Dollars and Other Brokerage Arrangements. Glasser LegalWorks, 2002 edition, 2001.

72 P. C. Lima. Wavelets: Uma introdução. Departamento de Matemática - ICEX - UFMG, 2003.

73 T. C. W. Lin. The new investor. UCLA Law Review, Vol. Review 678(no. 60), 2013.

74 T. C. W. Lin. The new financial industry. Alabama Law Review, Vol. Review 567(no. 65), 2014.

75 R. C. Littell, G. A. Milliken, W. W Stroup, and R. D. Wolfinger. Sas system for mixed models. Cary: Statistical Analysis System Institute, page 633, 2002.

76 C.-J. Lu, T.-S. Lee, and C.-C. Chiu. Financial time series forecasting using independent component analysis and support vector regression. Decision Support Systems, Vol. 47(no. 2):pp. 115-125, 2009.

77 Oliveira H. M. Análise de sinais para engenheiros: uma abordagem via wavelets. Rio de Janeiro, 244p, 2007.

78 L. Mahadeva and P. Robinson. Unit root testing to help model building. Handbooks in Central Banking, (no. 22), 2004.

79 O. Maimon and L. Rokach. Data Mining and Knowledge Discovery Handbook. Springer, 2nd edition, 2005.

80 S. Mallat. A wavelet tour of signal processing. USA, 577p, 1998.

81 B. B. Mandelbrot. Long-run linearity, locally gaussian process, h-spectra and infinite variances. International Economic Review, Vol. 10:p. 82, 1969.

82 B. B. Mandelbrot. Misbehavior of markets. 1st ed. edition, 2004.

83 B. B. Mandelbrot and R. L. Hudson. The (Mis)behavior of Markets: A Fractal View of Financial Turbulence. Basic Books, 2004.

84 B. B. Mandelbrot and J. W. Van Ness. Fractional brownian motions, fractional noises and applications. Vol. 10(no. 4):pp. 422-437, 1968. 
85 B. B. Mandelbrot and J. R. Wallis. Robustness of the rescaled range $\mathrm{r} / \mathrm{s}$ in the measurement of noncyclic long run statistical dependence. Water Resources Research, Vol. 5:p. 967, 1969.

86 G. A. Marcoulides. Discovering Knowledge in Data: an Introduction to Data Mining, volume 100. Wiley, 2005.

87 A. B. T. Martins, C. A. Taconeli, P. J. Ribeiro Jr., and A. C. A. Gonçalves. Análise de dados composicionais via árvores de regressão. Universidade Estadual de Maringá Departamento de Estatística, 2009.

$88 \mathrm{~J}$. Mercer. Functions of positive and negative type, and their connection with the theory of integral equations. Philosophical Transactions of the Royal Society A: Mathematical, Physical and Engineering Sciences, Vol. 209(no. 441-458):pp. 415-446, 1909.

89 D. Meyer. Support vector machines - the interface to libsvm in package e1071. FH Technikum Wien, Austria, 2017.

90 D. Meyer, E. Dimitriadou, K. Hornik, A. Weingessel, F. Leisch, C.-C. Chang, and C.C. Lin. e1071: Misc functions of the department of statistics, probability theory group. CRAN, 2017.

91 M. Misiti, Y. Misiti, G. Oppenheim, and J.-M. Poggi. Wavelet toolbox: for use with matlab. The Math Works, Inc., 1997.

92 T. M. Mitchell. Machine Learning: An Artificial Intelligence Approach, volume II. Morgan Kaufmann, 1st edition, 1997.

93 P. A. Morettin. Ondas e Ondaletas: da análise de Fourier à análise de ondaletas. São Paulo, Brazil, 272p, 1999.

94 P. A. Morettin. Econometria Financeira : Um Curso em Séries Temporais Financeiras. Blucher, 2nd edition, 2011.

95 P. A. Morettin. Mae-0518 - modelagem em séries temporais financeiras. IME/USP, 2014.

96 K. R. Muller, S. Mika, G. Ratsch, K. Tsuda, and B. Scholkopf. An introduction to kernelbased learning algorithms. IEEE Transactions on Neural Networks, Vol. 12:pp. 181-201, 2001.

97 A. Nag and A. Mitra. Forecasting daily foreign exchange rates using genetically optimized neural networks. Journal of Forecasting, Vol. 511(July):pp. 501-511, 2002.

98 G. Nason. wavethresh: Wavelets statistics and transforms. CRAN, 2016. 
99 G.P. Nason and T. Sapatinas. Wavelet packet transfer function modeling of nonstationary time series. Statistics and Computing, Vol. 12:pp. 45-56, 2002.

100 Guy Nason. wavethresh - wavelets statistics and transforms. CRAN - The Comprehensive $R$ Archive Network, page 308, 2016.

101 A. A. Neto and Araujo A. M. P. A contabilidade e a gestão baseada no valor. Unidersidad León - Espanha, page 43, 2000.

$102 \mathrm{H}$. Ni and H. Yin. Neurocomputing exchange rate prediction using hybrid neural networks and trading indicators. Neurocomputing, Vol. 72(no. 13-15):pp. 2815-2823, 2009.

103 M. Noronha. Análise Técnica: Teorias, Ferramentas e Estratégias. Editec - Livros Técnicos, Rio de Janeiro, 1a edition, 1995.

104 M. F. M. Osborne. Brownian motion in the stock market. Vol. 7:pp. 145-173, 1959.

105 Miles Osborne. Statistical Machine Translation, pages 912-915. Springer US, 2010.

106 Edgar E. P. Fractal market analysis : Applying chaos theory to investment and economics. John Wiley and Sons, Inc., 1994.

107 P. F. Pai and C. S. Lin. A hybrid arima and support vector machines model in stock price forecasting. Omega, Vol. 33(no. 6):pp. 497-505, 2005.

108 W. Palma. Long-Memory Time Series: Theory and Methods. New Jersey, 2007.

109 E. Panas. Long memory and chaotic models of prices on the london metal exchange. Resources Policy, Vol. 27:pp. 235-246, 2001.

110 A. Passerini. Kernel methods, multiclass classification and applications to computational molecular biology. Universita Degli Studi di Firenze, 2004.

111 D. B. Percival and A. T. Walden. Wavelet Methods for Time Series Analysis. Cambridge Series in Statistical and Probabilistic Mathematics. Cambridge University Press, 1st edition, 2006.

112 E. E. Peters and D. A. Hsieh. American finance association chaos and order in the capital markets: A new view of cycles, prices, and market volatility. Vol. 48:pp. 2041-2044, 2014.

113 M. Pontil and A. Veri. Support vector machines for 3d object recognition. IEEE Transactions on Pattern Analysis and Machine Intelligence, Vol. 20:pp. 637-646, 1998. 
114 B. Premanode. Prediction of nonlinear nonstationary time series data using a new digital filter and support vector regression. Imperial College London Department of Electrical and Electronic Engineering, 2013.

115 Cecilia M. Procopiuc. Projective Clustering, pages 806-811. Springer US, 2010.

116 Conhecimento Público. Free forex historical data. HistData.com, 2017.

117 R. Racine. Estimating the hurst exponent. pages 1-30, 2011.

118 J. B. Ramsey. Wavelets in economics and finance: Past and future. Studies in Nonlinear Dynamics and Econometrics, Vol. 6(no. 3), 2002.

119 G. R. Richards. The fractal structure of exchange rates: measurement and forecasting. Journal of International Financial Markets, Institutions and Money, Vol. 10:pp. 163-180, 2000.

120 V. M. Rivas, J. J. Merelo, P. A. Castillo, M. G. Arenas, and J. G. Castellano. Evolving rbf neural networks for time-series forecasting with evrbf. Information Sciences, Vol. 165(no. 3-4):pp. 207-220, 2004.

121 P. Roebuck. rwt - rice wavelet toolbox wrapper. CRAN - The Comprehensive R Archive Network, page 3, 2013.

122 Claude Sammut and Geoffrey I. Webb, editors. Mean Absolute Error, pages 652-652. Springer US, 2010.

123 Claude Sammut and Geoffrey I. Webb, editors. Mean Error, pages 652-652. Springer US, 2010.

124 B. Scholkopf. Learning with kernels. Journal of the Electrochemical Society, Vol. 129(November):p. 2865, 2002.

125 B. I. Settlements. Triennial central bank survey. foreign exchange turnover in april 2013: preliminary global results. Bank for International Settlements, (April):p. 24, 2013.

126 W. Sharpe. Portfolio theory and capital markets. 1970.

127 R. H. Shumway and D. S. Stoffer. Time Series Analysis and Its Applications with R Examples. Springer Texts in Statistics. Springer, 3rd edition, 2012.

128 S. G. Silva. Uso da tecnologia da informação no setor bancário: Um diagnóstico do uso da internet no banco do brasil, agência príncipe de joinville. Trabalho de Pós-Graduação em Administração e Gestão de Negócios Financeiros - UFRGS, page 64, 2007. 
129 J. K. Siqueira. Reconhecimento de voz contínua com atributos mfcc, ssch e pncc, wavelet denoising e redes neurais. Sistemas Maxwell - PUC-RIO, Certificação Digital n.o 0912874/CA, 2011.

130 A. J. Smola and B. Schölkopf. On a kernel-based method for pattern recognition, regression, approximation, and operator inversion. Algorithmica, Vol. 22(no. 1-2):pp. 211-231, 1998.

131 A. J. Smola and B. Schölkopf. A tutorial on support vector regression. 2004.

132 A. J. Smola, B. Schölkopf, P. L. Bartlett, and D. Schuurmans. Introduction to large margin classifiers. Advances in Large Margin Classifiers, pages 1-29, 2000.

133 M. K. P. So. Long-term memory in stock market volatility. Applied Financial Economics, Vol. 10(no. 5):pp. 519-524, 2000.

134 D. Sperandio, J. T. Mendes, and L. H. M. Silva. Cálculo Numérico - Características Matemáticas e Computacionais dos Métodos Numéricos. 2003.

135 F. E. H. Tay and L. J. Cao. Application of support vector machines in financial time series forecasting. Omega, Vol. 29(no. 4):pp. 309-317, 2001.

136 F. E. H. Tay and L. J. Cao. Modified support vector machines in financial time series forecasting. Neurocomputing, Vol. 48(no. 1-4):pp. 847-861, 2002.

137 P. Tenti. Forecasting foreign exchange rates using recurrent neural networks. Applied Artificial Intelligence, Vol. 10(no. 6):pp. 567-582, 1996.

138 T. Teräsvirta, C.-F. Lin, and C. W. J. Granger. Power of the neural network linearity test. Journal of Time Series Analysis, Vol. 14(no. 2):pp. 209-220, 1993.

139 Kai Ming Ting. Error Rate, pages 331-331. Springer US, 2010.

140 C. Torrence and G. A. Compo. A pratical guide to wavelets analysis. Bulletin of the American Meteorology Society, Vol. 79(no. 1):pp. 61-78, 1998.

141 G. Toussaint. Proximity graphs for nearest neighbor decision rule: Recent progress. Proc. of Interface, 2002.

142 R. S. Tsay. Analysis of Financial Time Series. University of Chicago, 2nd edition, 2005.

143 V. Vapnik, S. Golowich, and A. J. Smola. Support vector method for function approximation, regression estimation and signal processing. Advances in Neural Information Processing Systems 9, Vol. 9, 1996. 
144 V. Vapnik, S. Golowich, and A. J. Smola. Support vector method for multivariate density estimation. Advances in Neural Information Processing Systems, Vol. 12(MIT Press):pp. 659-665, 1999.

145 M. Vetterli and J. kovaćević. Wavelets and subband coding. New Jersey, 488p, 1995.

146 R. Von Sachs, G. P. Nason, and G. Kroisandt. Adaptive estimation of the evolutionary wavelet spectrum. Stanford, 1997.

$147 \mathrm{H}$. White. An additional hidden unit tests for neglected nonlinearity in multilayer feedforward networks. Proceedings of the International Joint Conference on Neural Networks, Vol. II:pp. 451-455, 1989.

148 R. D. Wolfinger. Covariance structure selection in general mixed models. Comunications in Statistics, Vol. 22:pp. 1079-1106, 1993.

149 Y. Yang, Geoffrey I. Webb, and X. Wu. Data Mining and Knowledge Discovery Handbook. Springer, 2nd edition, 2010.

150 J. Yim. Previsão de Séries de Tempo: Modelos ARIMA, Modelos Estruturais e Redes Neurais Artificiais. PhD thesis, Universidade de São Paulo, São Paulo, 2001.

151 S. Yousefi, I. Weinreich, and D. Reinarz. Wavelet-based prediction of oil prices. Chaos, Solitons and Fractals, Vol. 25(no. 2):pp. 265-275, 2005.

152 B. L. Zhang, R. Coggins, M. A. Jabri, D. Dersch, and B. Flower. Multiresolution forecasting for futures trading using wavelet decompositions. IEEE Transactions on Neural Networks, Vol. 12(no. 4):pp. 765-775, 2001.

153 B. L. Zhang and Z. Y. Dong. An adaptive neural-wavelet model for short term load forecasting. Electric Power Systems Research, Vol. 59(no. 2):pp. 121-129, 2001.

154 G. P. Zhang. Time series forecasting using a hybrid arima and neural network model. Neurocomputing, Vol. 50:pp. 159-175, 2003.

155 G. P. Zhang, B. E. Patuwo, and Hu M. Y. Forecasting with artificial neural networks: The state of the art. International Journal of Forecasting, Vol. 14:pp. 35-62, 1998.

156 Xinhua Zhang. Regularization, pages 845-849. Springer US, 2010.

$157 \mathrm{Li}$ Zhu, Yanxin Wang, and Qibin Fan. Modwt-arma model for time series prediction. Applied Mathematical Modelling, Vol. 38:pp. 1859-1865, 2014. 


\section{Anexo A - Métodos em R - Cálculo dos Testes de Normalidade e de Raiz Unitária, Correção de Dados Discrepantes, Técnicas de Denoising ou Wavelet Shrinkage e Persistência de Plots}

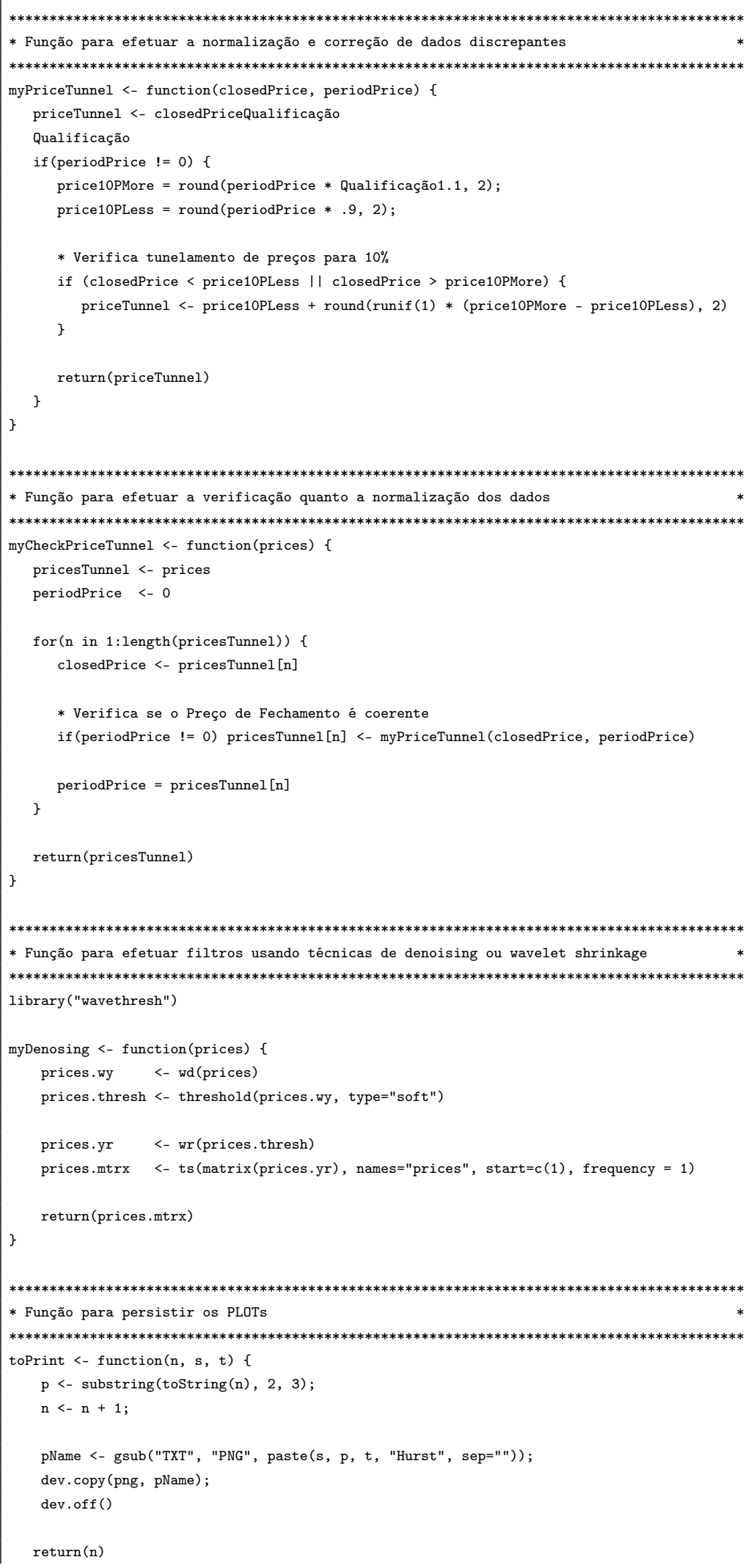




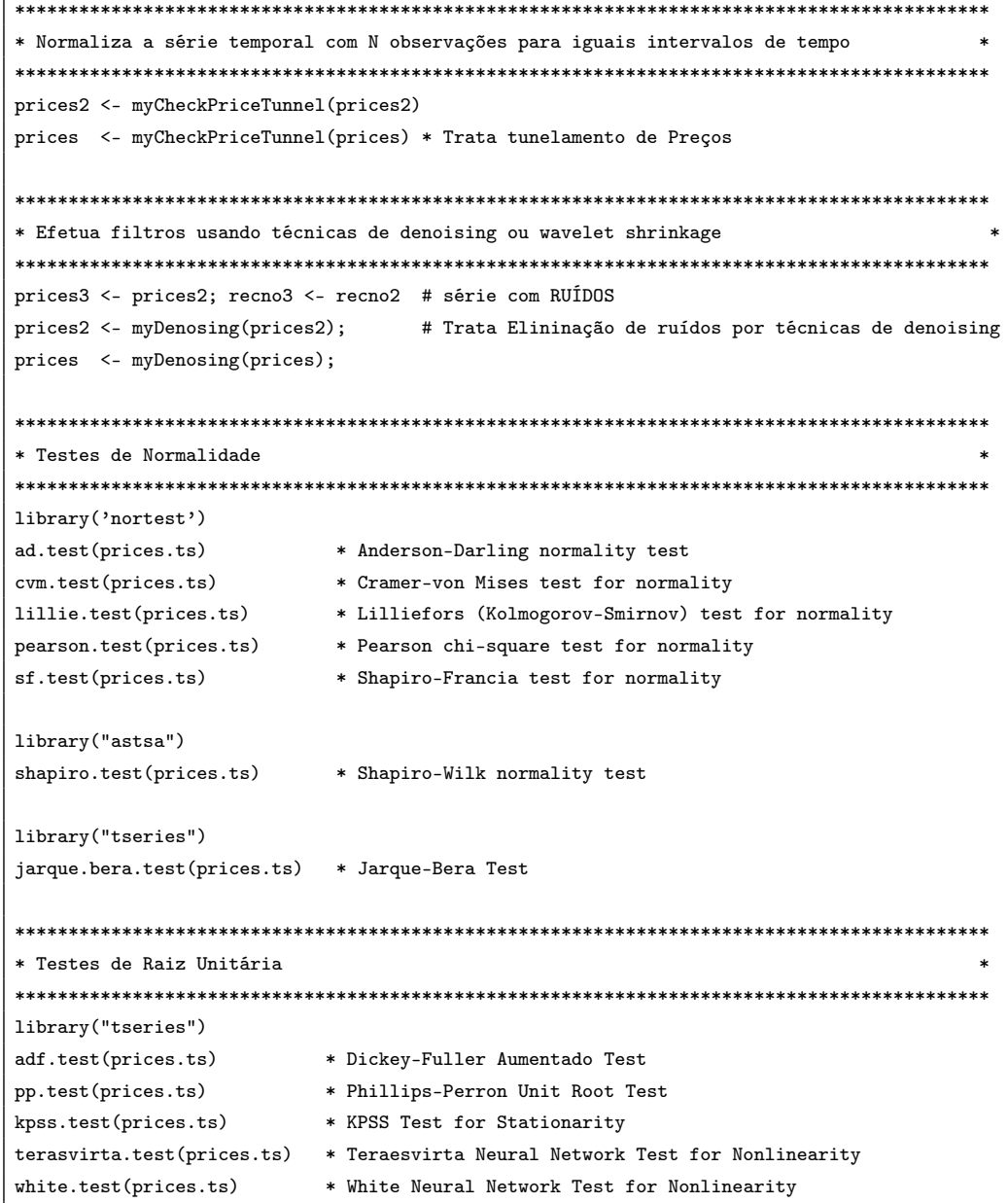


Anexo B - Métodos em R - Cálculo das Métricas MSE, RMSE, MAE, MPE, MAE, ACF1, AIC1, AICc1 e BIC1

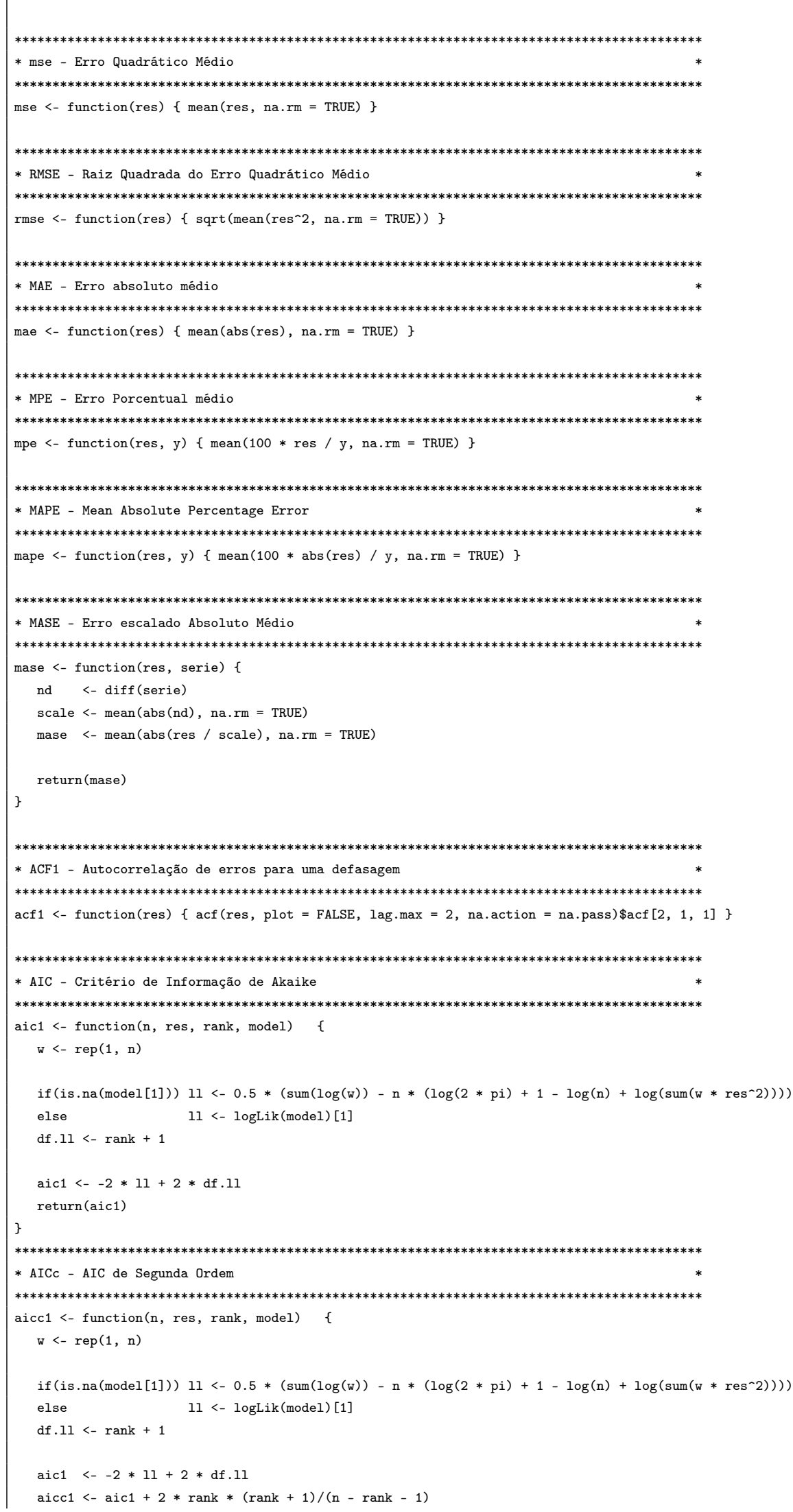




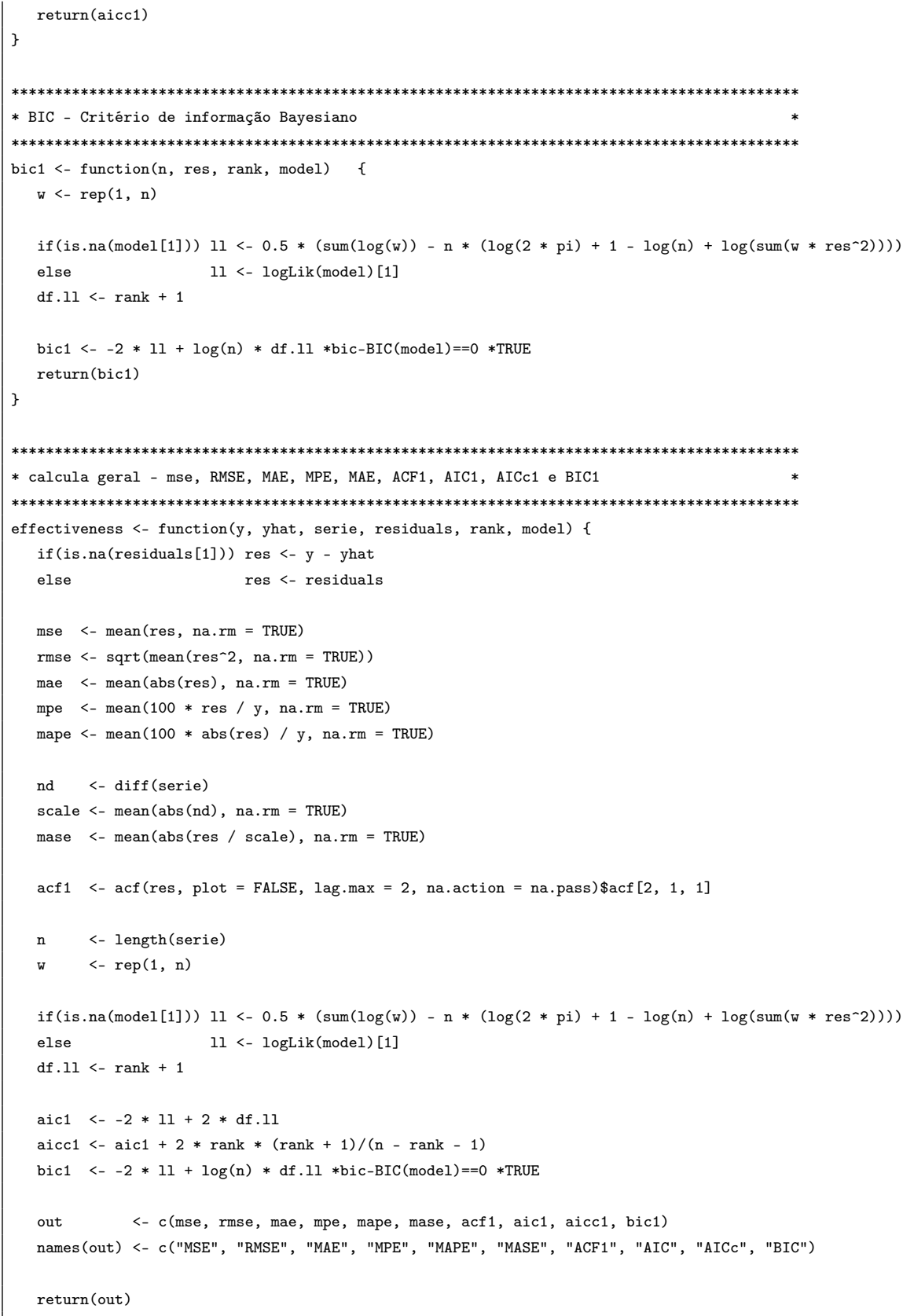


Anexo C - Métodos em R - Cálculo da Transformada wavelet e de sua Inversa

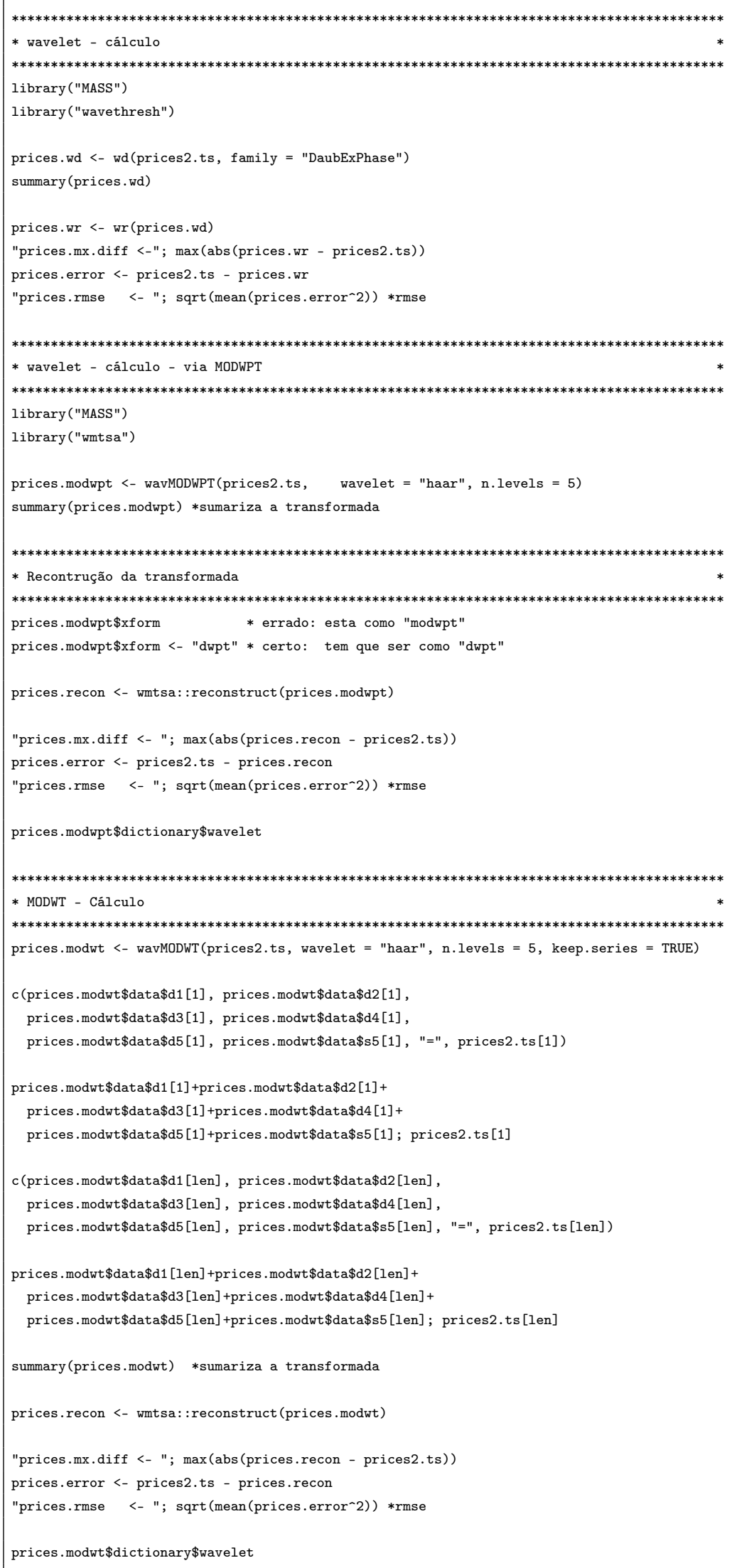


Anexo D - Métodos em R - Cálculo da Previsão dos Compontentes wavelet

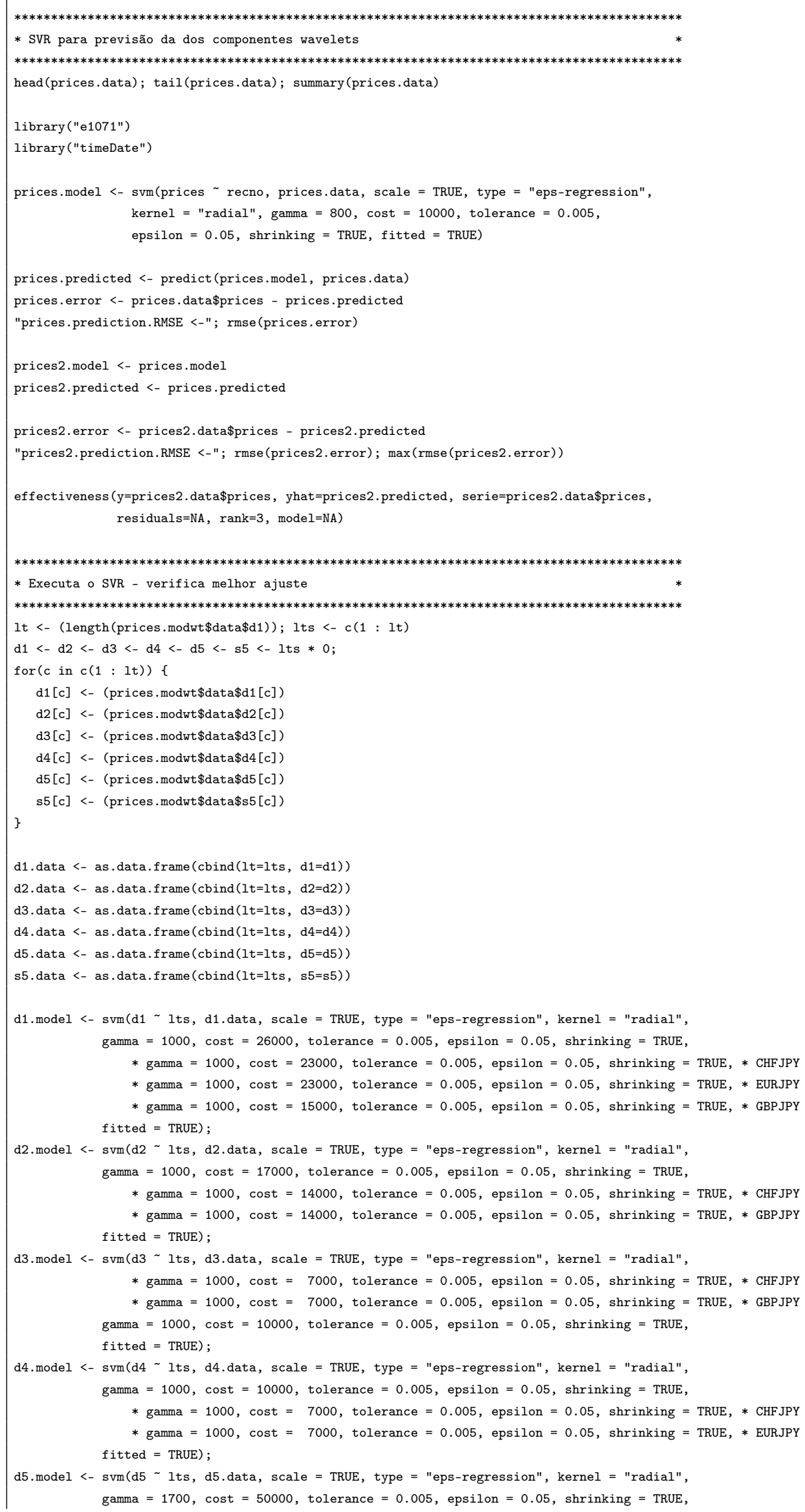




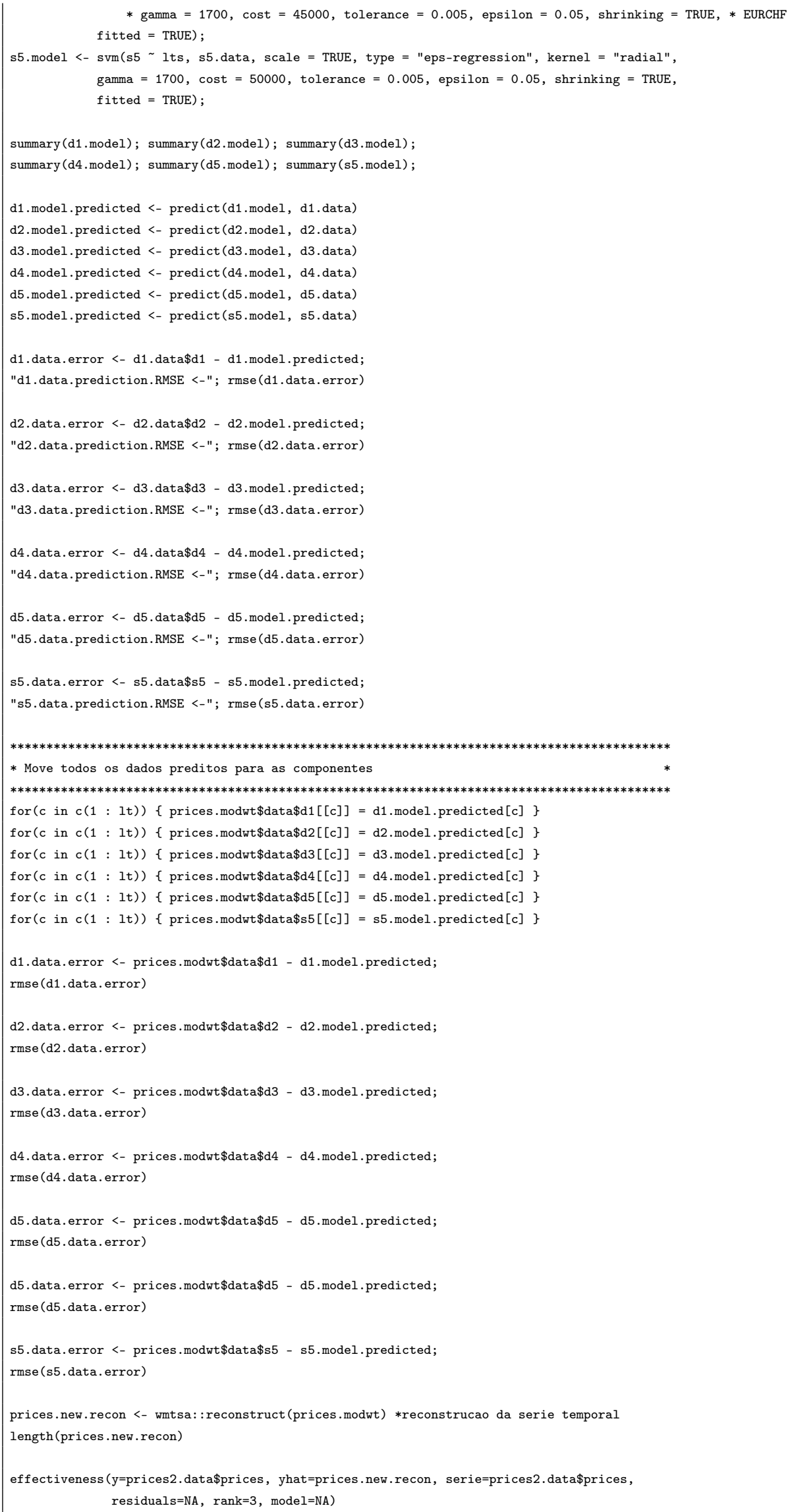


Anexo E - Métodos em R - Cálculo da Previsão dos Modelos Tradicionais e Comparação com o Modelo Proposto

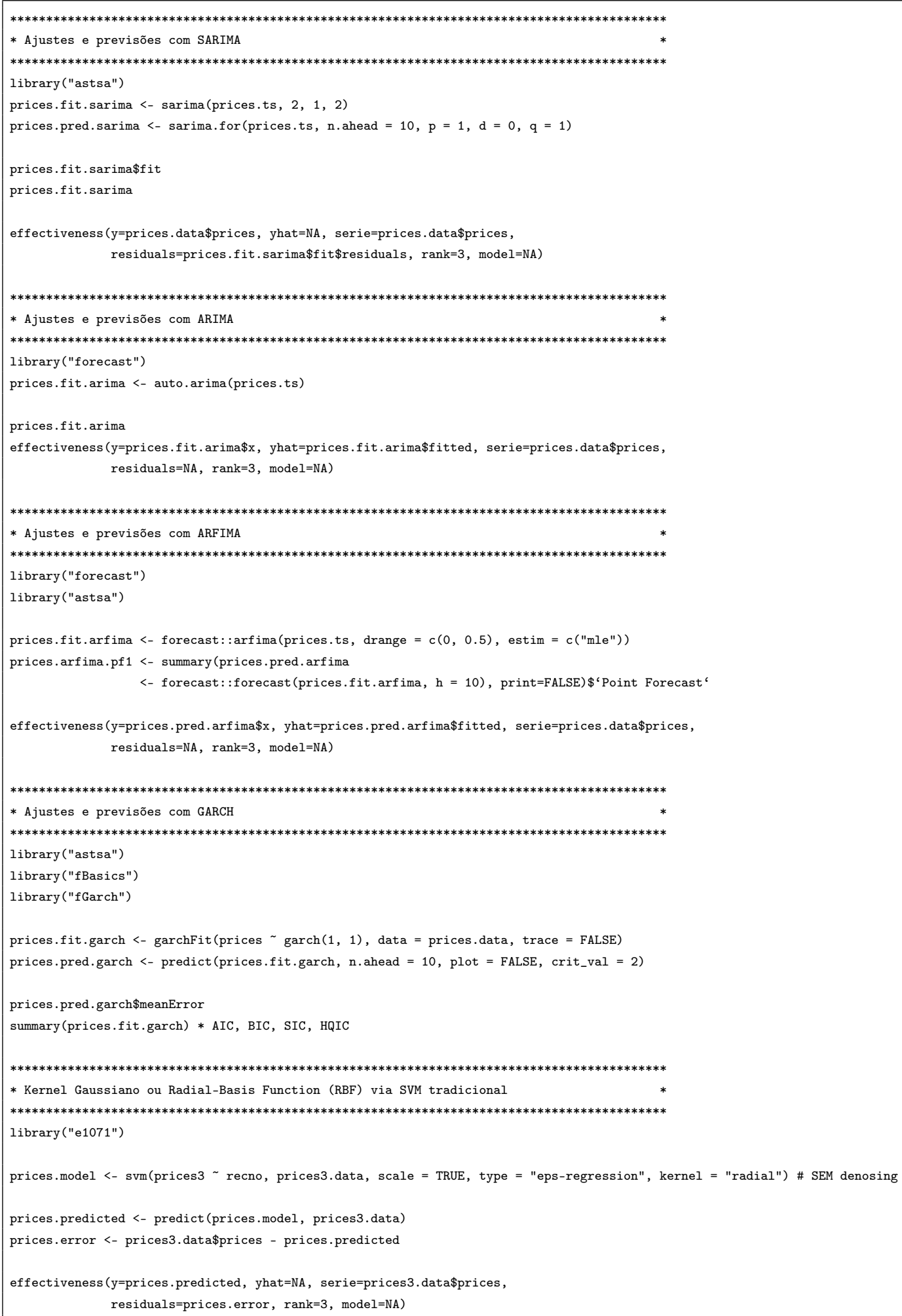


Anexo F - Métodos em R - Cálculo do Expoente de Hurst - Médias e Desvio Padrão

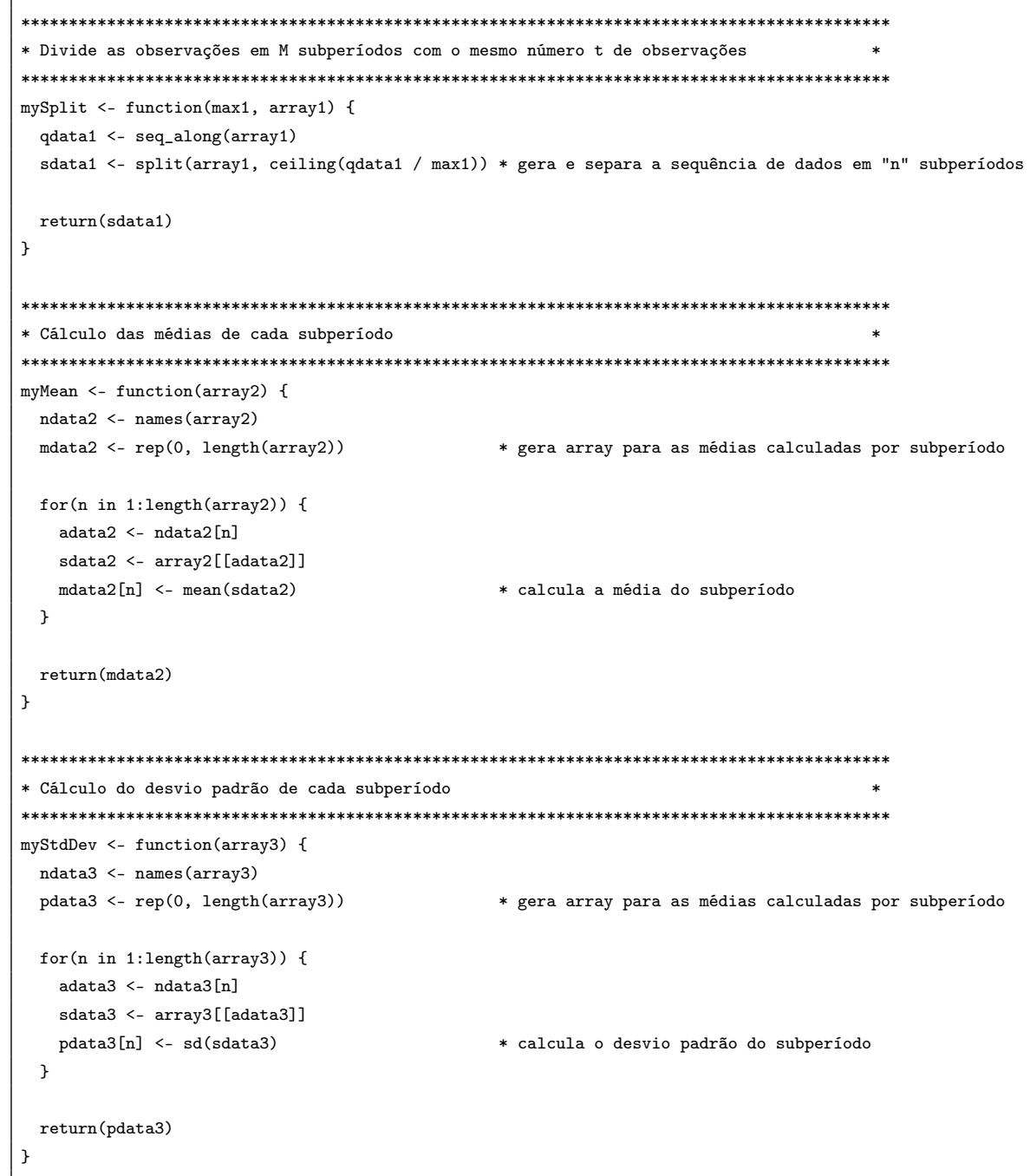




\section{Anexo G - Métodos em R - Cálculo do Expoente de Hurst por meio da Estatística R/S}

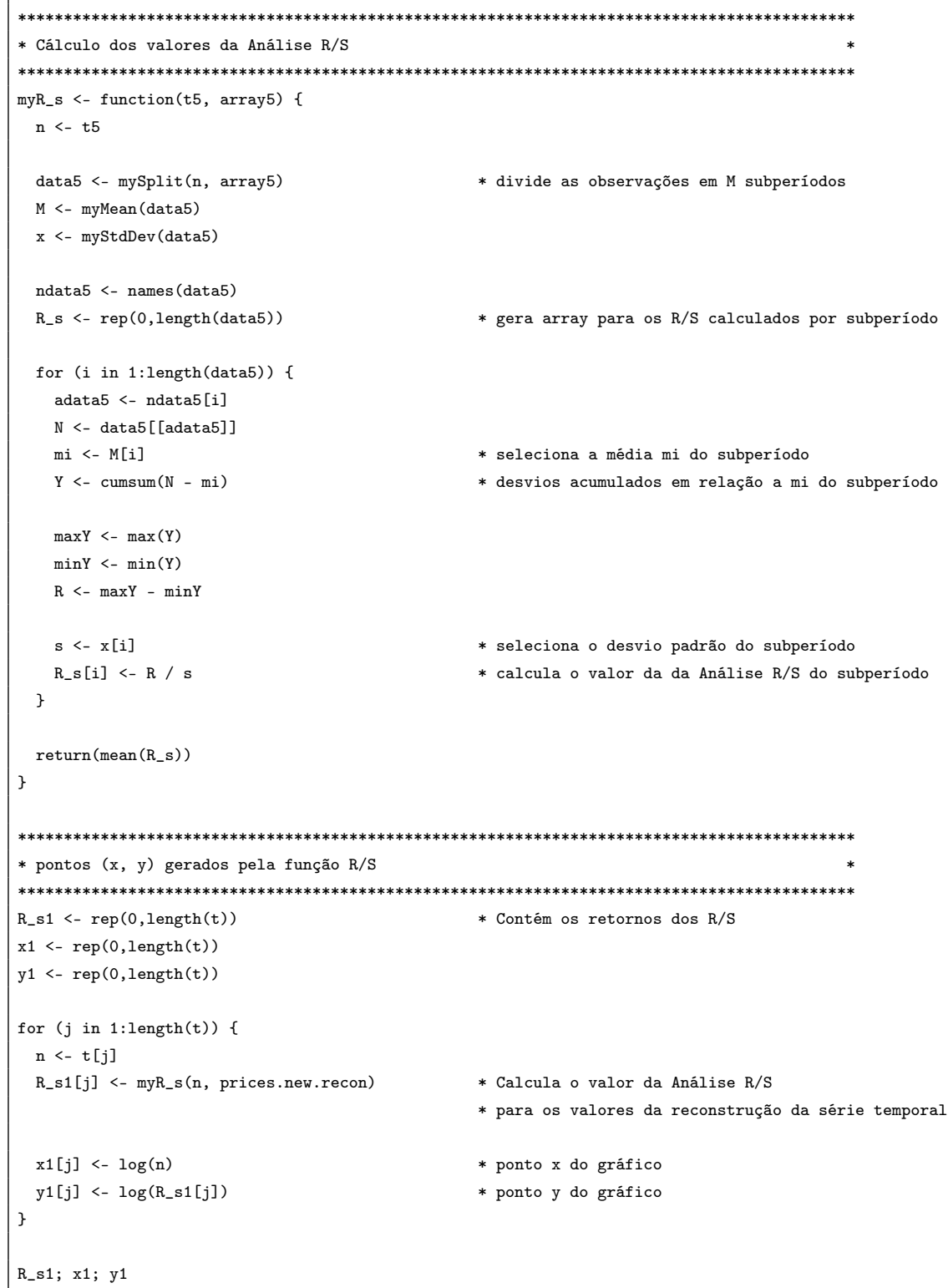


Anexo H - Métodos em R - Cálculo do Expoente de Hurst - Plot da Reta de Tendência e Cálculo do Valor de p-value

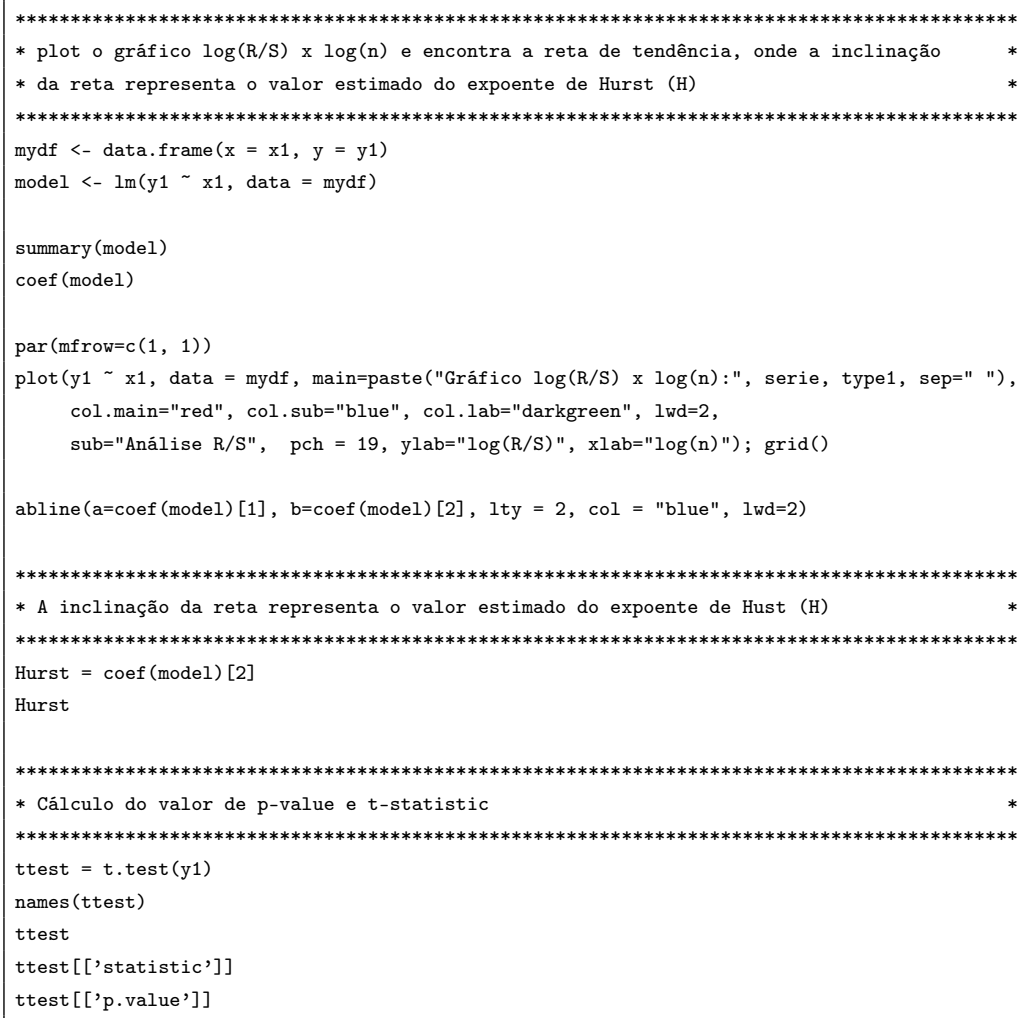




\section{Anexo I - Métodos em C\# - Cálculo da Divisão das Séries em Períodos de 15 min., 1 hora e 1 dia}

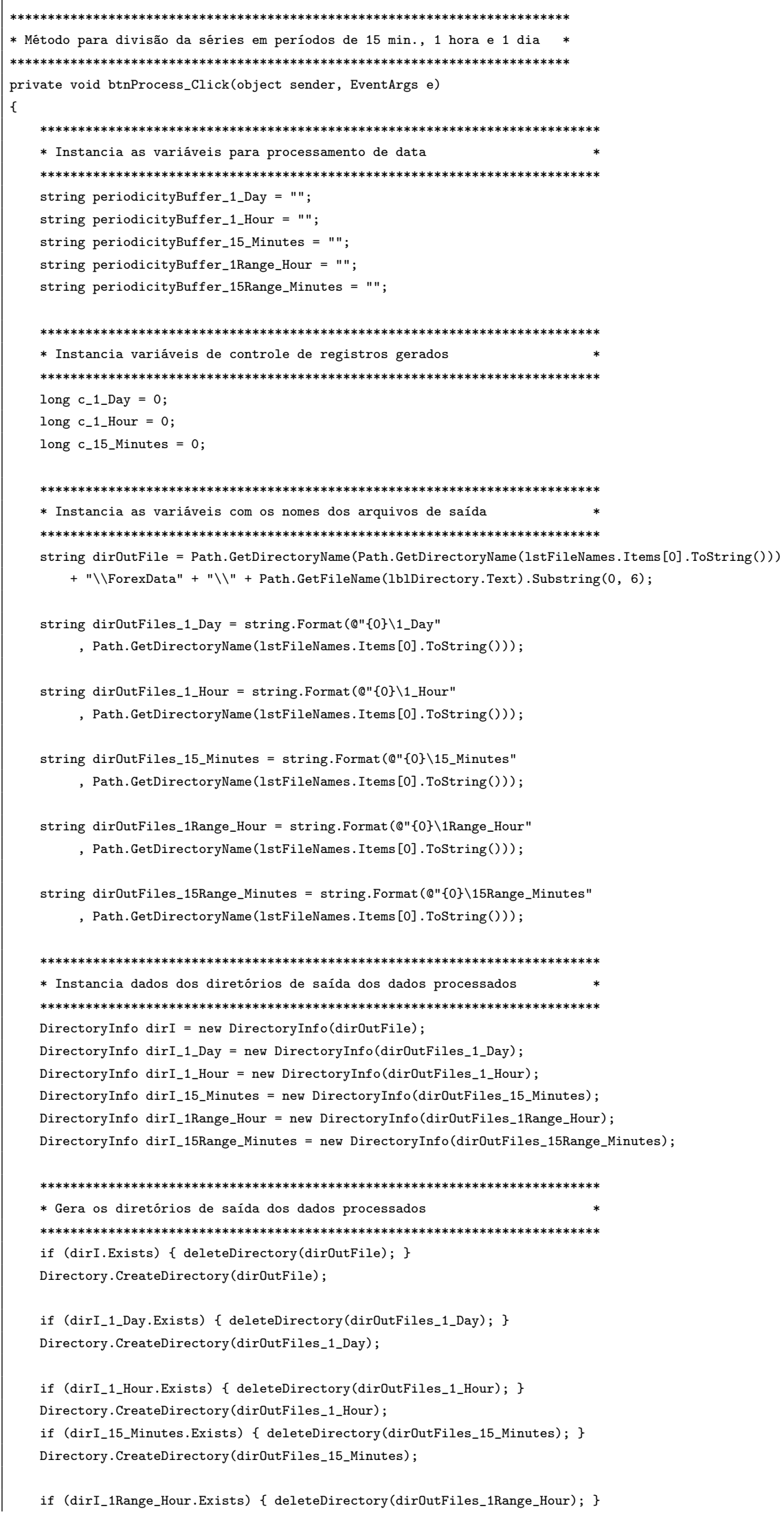


Directory.CreateDirectory (dirOutFiles_1Range_Hour);

if (dirI_15Range_Minutes.Exists) \{ deleteDirectory (dirOutFiles_15Range_Minutes); \}

Directory.CreateDirectory (dirOutFiles_15Range_Minutes);

$* * * * * * * * * * * * * * * * * * * * * * * * * * * * * * * * * * * * * * * * * * * * * * * * * * * * * * * * * * * * * * * * * * * * * * * * * * * * * * * *$

* Gera os nomes dos arquivos de saída dos dados processados *

$* * * * * * * * * * * * * * * * * * * * * * * * * * * * * * * * * * * * * * * * * * * * * * * * * * * * * * * * * * * * * * * * * * * * * * * * * * * * * * *$

string outFile1Day = string. Format $\left(\mathbb{Q} "\{0\} \backslash\{1\} \_1\right.$ _Day.txt", dirOutFile

Path.GetFileName (1blDirectory.Text).Substring $(0,6)$ );

string outFile1Hour = string.Format $\left(\mathbb{Q} "\{0\} \backslash\{1\} \_1 \_\right.$Hour.txt", dirOutFile , Path.GetFileName(lblDirectory.Text). Substring $(0,6)$ );

string outFile15Min $=$ string.Format $\left(\mathbb{Q} "\{0\} \backslash\{1\} \_15 \_\right.$Min.txt", dirDutFile , Path.GetFileName (lblDirectory. Text). Substring $(0,6)$ );

string outFile_1_Day = string.Format $\left(\mathcal{Q} "\{0\} \backslash\{1\} \_1 \_D a y \cdot t x t "\right.$, dirOutFiles_1_Day , Path.GetFileName(1blDirectory.Text).Substring $(0,6)$ );

string outFile_1_hour = string.Format (@" $\{0\} \backslash\{1\} \_1 \_H o u r . t x t "$, dirOutFiles_1_Hour , Path.GetFileName (lblDirectory.Text). Substring $(0,6))$;

string outFile_15_Minutes $=$ string.Format $\left(\varrho "\{0\} \backslash\{1\} \_15 \_\right.$Min.txt", dirOutFiles_15_Minutes , Path.GetFileName(1blDirectory.Text).Substring $(0,6)$ );

string outFile_1Range_hour $=$ string.Format $\left(\mathbb{Q} "\{0\} \backslash\{1\} \_1\right.$ Range_Hour.txt", dirOutFiles_1Range_Hour , Path.GetFileName(lblDirectory.Text).Substring $(0,6)$ );

string outFile_15Range_Minutes = string.Format $\left(\varrho "\{0\} \backslash\{1\} \_15\right.$ Range_Min.txt" , dirOutFiles_15Range_Minutes , Path.GetFileName(lblDirectory.Text).Substring $(0,6))$;

* Para cada arquivo contido na lista de arquivos a pr

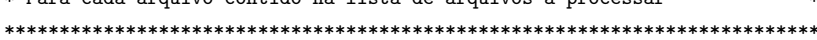

for (int $i=0 ; i<$ lstFileNames. Items.Count; $i++$ )

\{

string file $=$ lstFileNames.Items[i].ToString () ;

* Abre $\bigcirc$ arquivo da lista de arquivos a serem processados

using (StreamReader sr $=$ new $\operatorname{StreamReader}($ file $))$

\{

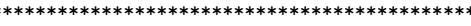

* Efetua a leitura de todo o arquivo e gera ARRAY com os registros

String buffer $=$ sr. ReadToEnd () ;

string [] lines = buffer.Split $\left({ }^{\prime} \backslash \mathrm{n}\right.$ ');

$* * * * * * * * * * * * * * * * * * * * * * * * * * * * * * * * * * * * * * * * * * * * * * * * * * * * * * * * * * * * * * * * * * * * * * * * * * * * * * *$

* Para cada linha lida do arquivo de trabalho

$* * * * * * * * * * * * * * * * * * * * * * * * * * * * * * * * * * * * * * * * * * * * * * * * * * * * * * * * * * * * * * * * * * * * * * * * * * * * * * *$

foreach (string line in lines)

\{

* Verifica se linha contem dados, gera ARRAY com as colunas do registro *

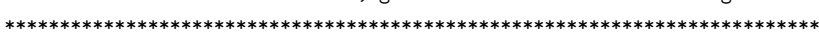

if (string. IsNullOrEmpty (line)) continue;

string [] fields = line.Split (';');

dos de Timestamp (Data + Horário) e Preço de Fechamento *

DateTime date $=$ Convert.ToDateTime(string.Format (" $\{0\}-\{1\}-\{2\}\{3\}:\{4\}:\{5\} "$

, fields [0].Substring $(0,4)$

, fields [0].Substring $(4,2)$

, fields [0].Substring $(6,2)$

, fields [0].Substring $(9,2)$

, fields [0].Substring $(11,2)$

, fields [0] .Substring $(13,2))$ );

double closedPrice $=$ Convert.. ToDouble $($ fields $[4]) ;$ 


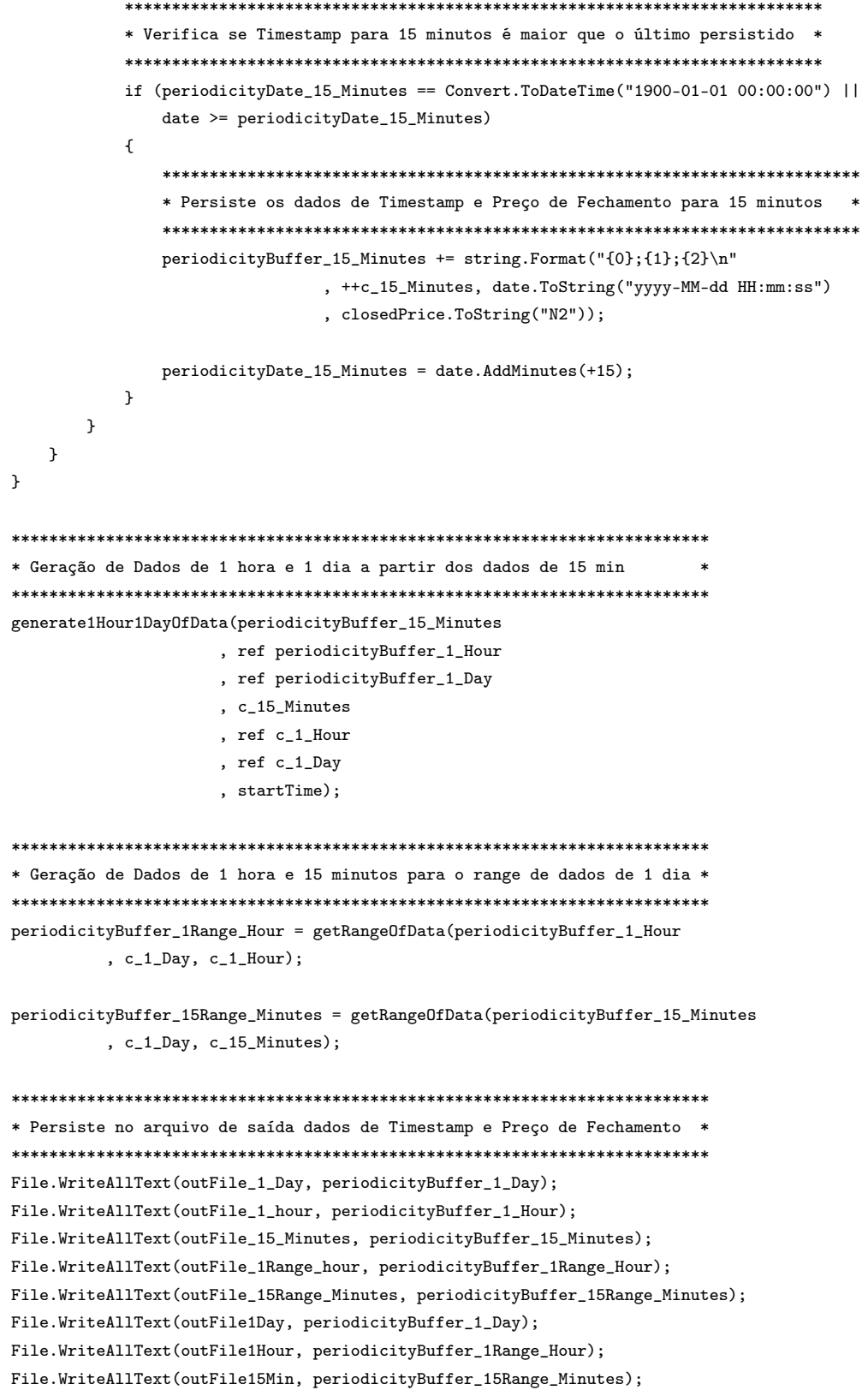


Anexo J - Métodos em C\# - Cálculo da Divisão dos Dados nos Períodos de 15 min., 1 hora e 1 dia

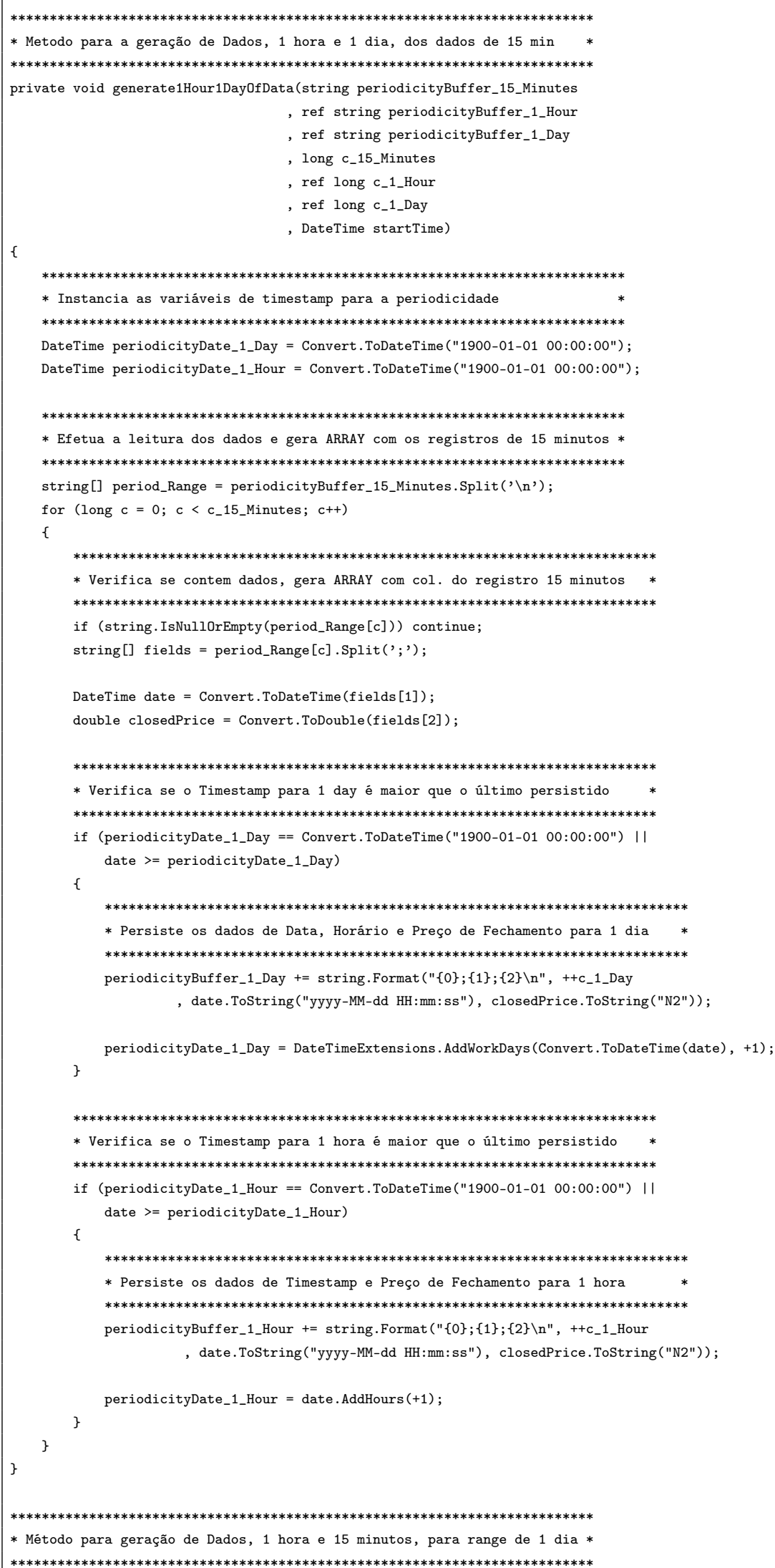




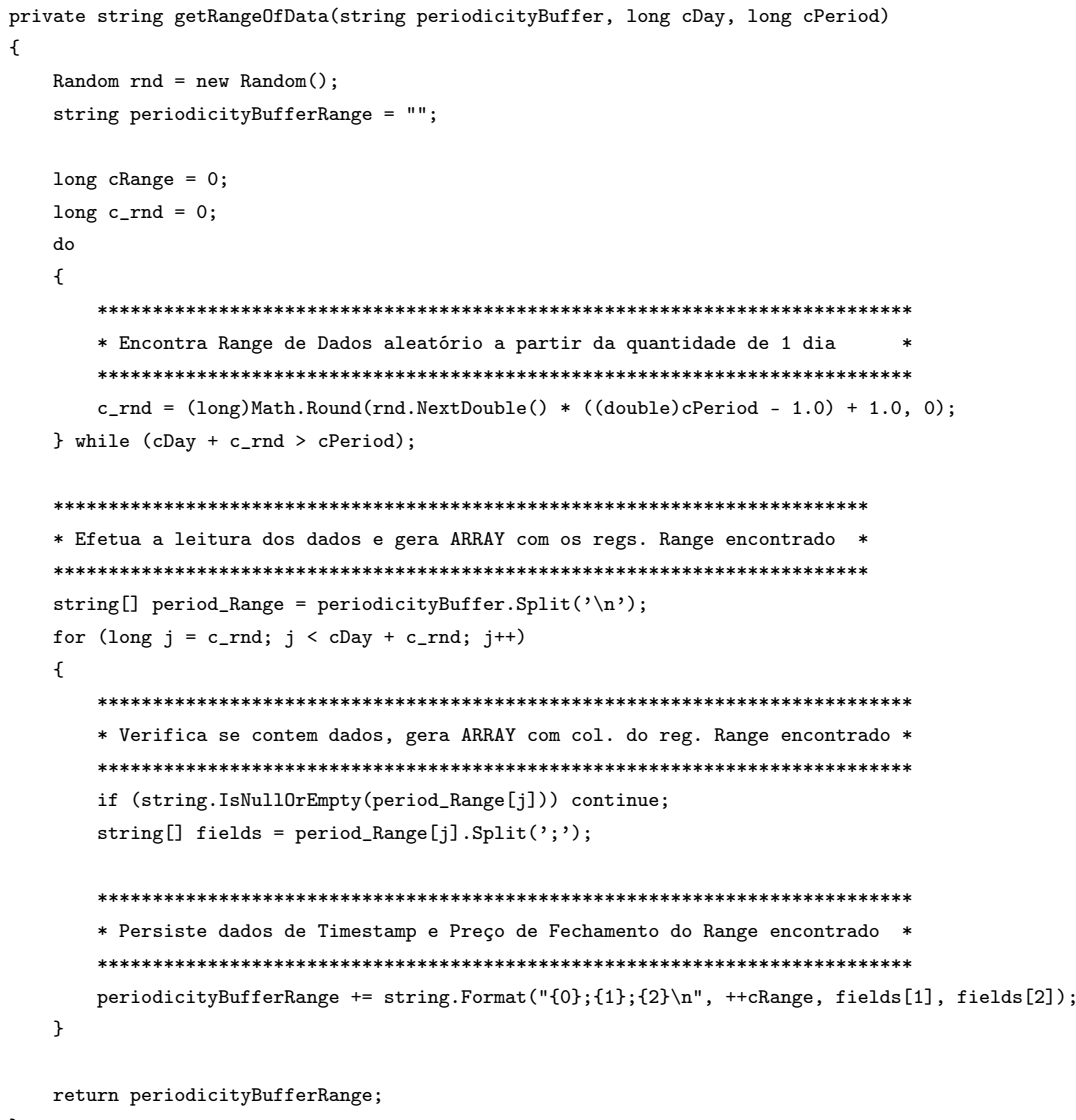

